Experimental Component Characterization, Monte-Carlo-Based Image Generation and Source Reconstruction for the Neutron Imaging System of the National Ignition Facility

Carlos A. Barrera, Michael J. Moran

August 29, 2007 
This document was prepared as an account of work sponsored by an agency of the United States Government. Neither the United States Government nor the University of California nor any of their employees, makes any warranty, express or implied, or assumes any legal liability or responsibility for the accuracy, completeness, or usefulness of any information, apparatus, product, or process disclosed, or represents that its use would not infringe privately owned rights. Reference herein to any specific commercial product, process, or service by trade name, trademark, manufacturer, or otherwise, does not necessarily constitute or imply its endorsement, recommendation, or favoring by the United States Government or the University of California. The views and opinions of authors expressed herein do not necessarily state or reflect those of the United States Government or the University of California, and shall not be used for advertising or product endorsement purposes.

This work was performed under the auspices of the U.S. Department of Energy by University of California, Lawrence Livermore National Laboratory under Contract W-7405-Eng-48. 


\title{
Experimental Component Characterization, Monte Carlo-Based Image Generation and Source Reconstruction for the Neutron Imaging System of the National Ignition Facility
}

\author{
by \\ Carlos Andres Barrera \\ Ingen (Universidad Pontificia Bolivariana, Colombia) 1998 \\ M.S. (University of California, Berkeley) 2004
}

A dissertation submitted in partial satisfaction of the requirements for the degree of Doctor of Philosophy in Engineering-Nuclear Engineering in the

GRADUATE DIVISION

of the UNIVERSITY OF CALIFORNIA, BERKELEY

Committee in charge:

Prof. Edward Morse, Chair

Prof. Donald Olander

Prof. Marjorie Shapiro

Michael Moran

Fall 2007 
The dissertation of Carlos Andres Barrera is approved:

\begin{tabular}{rr}
\hline Chair & Date \\
\hline & Date \\
\hline & \\
\hline & Date \\
\hline & Date
\end{tabular}

University of California, Berkeley

Fall 2007 
Experimental Component Characterization, Monte Carlo-Based Image Generation and Source Reconstruction

for the Neutron Imaging System of the National Ignition Facility

Copyright 2007

by

Carlos Andres Barrera 


\author{
Abstract \\ Experimental Component Characterization, Monte Carlo-Based \\ Image Generation and Source Reconstruction \\ for the Neutron Imaging System of \\ the National Ignition Facility \\ by \\ Carlos Andres Barrera \\ Doctor of Philosophy in Engineering-Nuclear Engineering \\ University of California, Berkeley \\ Prof. Edward Morse, Chair
}

The Neutron Imaging System (NIS) is one of seven ignition target diagnostics under development for the National Ignition Facility. The NIS is required to record hot-spot (13-15 MeV) and downscattered (6-10 MeV) images with a resolution of 10 microns and a signal-to-noise ratio (SNR) of 10 at the $20 \%$ contour. The NIS is a valuable diagnostic since the downscattered neutrons reveal the spatial distribution of the cold fuel during an ignition attempt, providing important information in the case of a failed implosion. The present study explores the parameter space of several line-of-sight (LOS) configurations that could serve as the basis for the final design. Six commer- 
cially available organic scintillators were experimentally characterized for their light emission decay profile and neutron sensitivity. The samples showed a long lived decay component that makes direct recording of a downscattered image impossible. The two best candidates for the NIS detector material are: EJ232 (BC422) plastic fibers or capillaries filled with EJ399B. A Monte Carlo-based end-to-end model of the NIS was developed to study the imaging capabilities of several LOS configurations and verify that the recovered sources meet the design requirements. The model includes accurate neutron source distributions, aperture geometries (square pinhole, triangular wedge, mini-penumbral, annular and penumbral), their point spread functions, and a pixelated scintillator detector. The modeling results show that a useful downscattered image can be obtained by recording the primary peak and the downscattered images, and then subtracting a decayed version of the former from the latter. The difference images need to be deconvolved in order to obtain accurate source distributions. The images are processed using a frequency-space modified-regularization algorithm and low-pass filtering. The resolution and SNR of these sources are quantified by using two surrogate sources. The simulations show that all LOS configurations have a resolution of 7 microns or better. The $28 \mathrm{~m}$ LOS with a $7 \times 7$ array of 100 -micron minipenumbral apertures or 50-micron square pinholes meets the design requirements and is a very good design alternative.

Prof. Edward Morse

Dissertation Committee Chair 
Once more, to my parents and sister,

for always being there.

I love you so much. 


\section{Contents}

List of Figures iv

List of Tables vii

1 Introduction $\quad 1$

1.1 Nuclear Fusion Fundamentals . . . . . . . . . . . . . . . . . . 2

1.2 Inertial Confinement Fusion Concepts . . . . . . . . . . . . . . . . . . 11

1.3 The National Ignition Facility . . . . . . . . . . . . . . . . . . . 17

1.4 Structure of the Study . . . . . . . . . . . . . . . 25

2 Neutron Imaging Principles $\quad 27$

2.1 Concept and Neutronics . . . . . . . . . . . . . . . . . . 28

2.2 Advantages and Disadvantages . . . . . . . . . . . . . . . . . . . . . . . . . . . . . 37

2.3 A Typical Line-of-Sight . . . . . . . . . . . . . . . . . . . . . . . . . . . . . . . . . . .

2.3.1 Aperture . . . . . . . . . . . . . . . . . 39

2.3.2 Scintillator Array . . . . . . . . . . . . . . . . . . . . . 45

2.3.3 Optical System . . . . . . . . . . . . . . . . . . . . . . . 49

2.3.4 Digital Camera . . . . . . . . . . . . . . . 50

2.4 Historical Development . . . . . . . . . . . . . . . . . 50

3 Scintillator Characterization $\quad 53$

3.1 Scintillator Samples . . . . . . . . . . . . . . . . . 54

3.2 Light Output Decay Measurement . . . . . . . . . . . . . . . . 56

3.3 Light Output Color Filtering . . . . . . . . . . . . . . . 66

3.4 Absolute Sensitivity and Photon Gain . . . . . . . . . . . . . 69

3.5 Fiber Array Light Emission Distribution . . . . . . . . . . . . . . 82

3.6 Scintillator Selection . . . . . . . . . . . . . . . . 84

4 Model-based Neutron Imaging $\quad 86$

4.1 The End-to-End Model . . . . . . . . . . . . . . . . . . 87 
4.2 Neutron Source Distributions _ . . . . . . . . . . . . . . 88

4.3 MCNPX . . . . . . . . . . . . . . . . . . 89

4.3.1 Preliminary Simulations . . . . . . . . . . . . . . . . . . . 91

4.3 .2 Sample Input Deck . . . . . . . . . . . . . . . . . . . . . . . 98

4.4 Point Spread Functions . . . . . . . . . . . . . . . . . . . . . 101

4.5 Image Formation with Scintillator Decay . . . . . . . . . . . . . . 105

4.5.1 Zero Degradation Case . . . . . . . . . . . . . . . . 107

4.5.2 Image Subtraction Algorithm . . . . . . . . . . . . . . . . . 108

4.5.3 Raw Images . . . . . . . . . . . . . . . . . . . . 111

5 Digital Image Processing $\quad 114$

5.1 Mathematical Treatment . . . . . . . . . . . . . 115

5.2 Implementation . . . . . . . . . . . . . . . . . . . . . . . . . 121

5.2.1 Power Spectra and Modulation Transfer Function . . . . . . . 122

5.2 .2 Source Reconstruction . . . . . . . . . . . . . . . . 126

5.2.3 Low-Pass Filtering . . . . . . . . . . . . . . . . . . . . 133

5.2 .4 Field of View Variation . . . . . . . . . . . . . . . . . 139

6 Image Quality 143

6.1 Image Quality and Design Requirements . . . . . . . . . . . . . . . 144

6.2 Image Noise . . . . . . . . . . . . . . . . . . . . . . . 146

6.3 Resolution . . . . . . . . . . . . . . . . . . . 150

6.4 Signal-to-Noise Ratio . . . . . . . . . . . . . . . . . . . 153

6.5 Surrogate Sources . . . . . . . . . . . . . . . . . . . 155

7 Concluding Remarks and Suggestions for Further Work 160

7.1 Scintillator Characterization . . . . . . . . . . . . . . . 161

7.2 End-to-End Model . . . . . . . . . . . . . . . . . . . . 161

7.3 Image Processing and Source Recovery . . . . . . . . . . . . . . . . . 162

7.4 Image Quality and Aperture Selection . . . . . . . . . . . . . . 163

7.5 Areas of Further Work . . . . . . . . . . . . . . . . . . . 164

$\begin{array}{ll}\text { References } & 170\end{array}$ 


\section{List of Figures}

1.1 Binding Energy per Nucleon . . . . . . . . . . . . . . . . . 4

1.2 Ion-Ion Potential Diagram . . . . . . . . . . . . . . 5

1.3 Sigma-Vee vs. Temperature Plot . . . . . . . . . . . . . . 8

1.4 NIF Capsule Design . . . . . . . . . . . . . . . . . . . . . . . . . . . . . . . . . . .

$1.5 \mathrm{ICF}$ Steps . . . . . . . . . . . . . . . . . . . . . . . . . . . . . . . . . . . . . . . . .

1.6 NIF Hohlraum . . . . . . . . . . . . . . . . . . . . . . . . . . 18

1.7 Laser Pulse Profile . . . . . . . . . . . . . . . . . . . . . . . . . 19

1.8 NIF Building . . . . . . . . . . . . . . . . . . . . 21

1.9 NIF Laser Beamline . . . . . . . . . . . . . . . . . . . . . 22

1.10 Laser Glass . . . . . . . . . . . . . . . . . . . . . . . 23

2.1 NIF Target Neutron Spectra . . . . . . . . . . . . . . . . . . 29

2.2 Neutron Elastic Scattering Diagram . . . . . . . . . . . . . . . 30

2.3 Ellastically Scattered Neutron Energy Plot . . . . . . . . . . . . . . 31

2.4 Legendre Polynomials Plots . . . . . . . . . . . . . . . . . . . . . . . . . 32

2.5 Simulated $P_{6}$ Asymmetry Density Plot . . . . . . . . . . . . . . . . 34

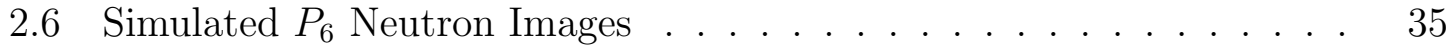

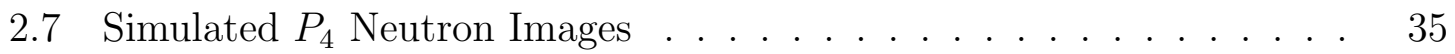

$2.840 \mathrm{~m} \mathrm{nToF}$ and Neutron Spectrum . . . . . . . . . . . . . . 36

2.9 Basic Pinhole Camera . . . . . . . . . . . . . . . . . . . 38

2.10 Basic Components of a Neutron Imaging System . . . . . . . . . . . . 39

2.11 Penumbral and Annular Apertures . . . . . . . . . . . . . . . . . . . . . 42

2.12 Pinhole Apertures . . . . . . . . . . . . . . . . . . . . . . . . . . . . . . . . 44

2.13 Scintillator Afterglow Image Contamination . . . . . . . . . . . . . . 47

3.1 TCPC Experimental Setup . . . . . . . . . . . . . 58

3.2 EJ232 Raw Data Light Emission Decay . . . . . . . . . . . . . . . 59

3.3 EJ232 Fitted Light Emission Decay . . . . . . . . . . . . . . . 60

3.4 Decay Curves for DT neutrons $1000 \mathrm{~ns} \ldots \ldots$. . . . . . . . . . . . 61

3.5 EJ399B Multisource Light Emission Decay . . . . . . . . . . . . 63 
3.6 Decay Curves for DT neutrons $100 \mathrm{~ns} \ldots \ldots \ldots \ldots$

3.7 Emission Spectra of BC422 and BC509 . . . . . . . . . . . 66

3.8 Electronic Level Structure for an Aromatic Molecule . . . . . . . . . 67

3.9 EJ399A Filtered Light Emission Decay . . . . . . . . . . . . . . . 68

3.10 APD Pulse Height Spectra for DT and DD . . . . . . . . . . . 71

3.11 Absolute Sensitivity Measurement Electronic Block Diagram . . . . . 72

3.12 Geometry detail for $\bar{\Delta}_{s c i} \ldots \ldots \ldots \ldots \ldots \ldots$

3.13 Experimental Relative Response of NE102 _ . . . . . . . . . . . 80

3.14 Relative Response of NE102 _ . . . . . . . . . . . . . . . . . 81

3.15 Plastic Fiber Array Light Output Angular Distribution . . . . . . . 85

4.1 NIS End-to-End Model Block Diagram . . . . . . . . . . . . . . 87

$4.2 P_{6}$ Neutron Source Distributions . . . . . . . . . . . . . . . 90

4.3 Aperture Scattering Contribution Simulations . . . . . . . . . . 93

4.4 Energy Deposition Plots of $14 \mathrm{MeV}$ Protons on EJ232 . . . . . . . . 95

4.5 Energy Deposition Plots of $14 \mathrm{MeV}$ Neutrons on EJ232 . . . . . . . . 96

4.6 Energy Deposition Plots of $14 \mathrm{MeV}$ Neutrons on Actual Detector Array 97

4.7 Point Spread Fucntions at $40 \mathrm{~m} \ldots \ldots \ldots$

4.8 Point Spread Fucntion Lineouts . . . . . . . . . . . . . . . . 103

4.9 EJ232 Decay Profile and Integrated Emission _ . . . . . . . . . 106

4.10 Primary and Downscattered Sources with $40 \mathrm{~m}$ Emission Fraction . . 108

4.11 Subtracted and 6 - $10 \mathrm{MeV}$ Sources Comparison . . . . . . . . . . 109

4.12 Image Subtraction Algorith Block Diagram . . . . . . . . . . . . . . 110

4.13 Raw Image Summary at $40 \mathrm{~m} \ldots \ldots$

4.14 Raw Image Summary at $28 \mathrm{~m} \ldots \ldots$

5.15 -micron Square Pinhole PSF Power Spectrum . . . . . . . . . . . 123

5.2 Power Spectra of the PSFs at $40 \mathrm{~m} \ldots \ldots \ldots \ldots$

5.3 Modulation Transfer Function of the $40 \mathrm{~m}$ PSFs . . . . . . . . . . . 125

5.4 Modulation Transfer Function of the $40 \mathrm{~m}$ Difference Downscattered Images . . . . . . . . . . . . . . . . . . . . . 126

5.5 MTF of the 5-micron Square Pinhole Difference Downscattered Source 128

5.6 5-micron Square Pinhole Reconstructed Downscattered Source . . . . 130

5.7 Penumbral and Annular Apertures Decoding Functions . . . . . . . 132

5.8 MTF of the Annular Autocorrelation Recovered Downscattered Source 133

5.9 Autocorrelation Recovered Downscattered Source . . . . . . . . . . 134

5.10 Mini-penumbral Autocorrelation Results . . . . . . . . . . . . . 135

5.11 50-micron Square Pinhole Filtered Recovered Sources . . . . . . . . 136

5.12 Filtered Recovered Downscattred Sources at $40 \mathrm{~m}$. . . . . . . . . . 137

5.13 Filtered Recovered Downscattred Sources at $28 \mathrm{~m}$. . . . . . . . 138

$5.14 \mathrm{FoV}$ Variation for $40 \mathrm{~m}$ LOS . . . . . . . . . . . . . . . . . 140

$5.15 \mathrm{FoV}$ Variation for $28 \mathrm{~m}$ LOS . . . . . . . . . . . . . . . . . 140 
5.1650 -micron Square Pinhole FoV Variation . . . . . . . . . . . . . . 142

6.1 Direct Transmission Histograms . . . . . . . . . . . . . . . . . . . . 149

6.2 Deconvolved Direct Transmission Histograms . . . . . . . . . . . . . . 150

6.350 -micron Square Pinhole Resolution Test . . . . . . . . . . . . . . . 152

$6.420 \%$ Contour of Square Pinhole Sources . . . . . . . . . . . . . . . 155

6.5 Image Quality Surrogate Sources . . . . . . . . . . . . . . . . 156

6.6 50-micron Square Pinhole Filtered Slab Sources . . . . . . . . . . . . 157

6.7 100-micron Mini-Penumbral Aperturee Gap Slab Lineouts . . . . . . 158

7.1 100-micron Mini-penumbral Array PSFs . . . . . . . . . . . . . . . 167

7.2 100-micron Mini-penumbral Array Recovered Downscattered Source . 168 


\section{List of Tables}

3.1 Selected Scintillators Basic Features . . . . . . . . . . . . . 55

3.2 Scintillator Decay Constabts . . . . . . . . . . . . . . . 62

3.3 Band-Pass Filter List . . . . . . . . . . . . . . . . . . . . 68

3.4 DD Absolute Sensitivity Experimental Data . . . . . . . . . . . 73

3.5 DT Absolute Sensitivity Experimental Data . . . . . . . . . . . . . 74

3.6 Sensitivity and Photon Gain Results . . . . . . . . . . . . . . . . . 79

3.7 Relative Sensitivity Results . . . . . . . . . . . . . . . . . . 79

3.8 Fiber Array Angular Distribution Experimental Data . . . . . . . . . 84

4.1 Apparent Aperture Width and Equivalent Diameter . . . . . . . . . . 104

4.2 EJ232 Light Emission Fractions for 28 and $40 \mathrm{~m}$. . . . . . . . . . 106

6.1 Aperture SNR Summary . . . . . . . . . . . . . . . . . 159 


\section{Acknowledgments}

I would like to thank my parents for their support and understanding all these years. Now I can finally say to them that I will not be a student any more, and that when I grow up I will be a Doctor in Nuclear Engineering.

To Professor Morse thank you for his advice, knowledge and humor, they made the rest of the trip even more enjoyable than the first part. I am sure that if I had not met him that fateful December 2000 afternoon, I would be still working in some obscure experiment at a Midwest school. Thank you for taking a chance on me.

To Mike Moran at the Lawrence Livermore National Laboratory I will be eternally in debt for all his time and guidance, for his invaluable love for grammar and syntax, and for knowing what to do and when to do it.

Also at LLNL I must recognize the help and advice of Nino Landen, Dick Lerche, Tom Phillips and Dan Kalantar, all of them fine scientists and mentors. Special thanks to Steve Hatchett and Charlie Cerjan for their help generating, processing and interpreting the hydrodynamic simulations. My gratitude also goes to Loeulla Gregg for her help with paperwork and administrative procedures. The people of the SEGRF program, that provided me with the means to conduct this work and get a taste of life in a world-class scientific institution. My special gratitude goes to Joanna Allen, Paul Dickinson and Harry Radousky.

I want to thank Laurent Disdier at CEA fom sharing his knowledge and experience in neutron imaging with me, as well as for letting me join his experimental team a 
couple of times at LLE. It was a great experience indeed.

At the Nuclear Engineering Department I have to thank again and again Lisa Zemelman, who knows how to save the day and kept me on track though the years. Thank you also to all the wonderful people at the SISS, who helped me be in this country legally and understand the minutia of immigration law.

Finally, a very special thank you to the Take me Out guys, for understanding and keeping me sane while writing. To Ed Decker goes my endless gratitude and admiration, it was a pleasure.

This work was performed under the auspices of the U.S. Department of Energy by the University of California, Lawrence Livermore National Laboratory under contract No. W-7405-Eng-48. 


\section{Chapter 1}

\section{Introduction}

These are certainly important times for the nuclear fusion community, the more and more immediate threat of global warming, as well as, the depletion of fossil fuel reserves around the world, are inviting people to reconsider nuclear energy as a viable alternative, fueling a nuclear renaissance after more than 20 years of quiet, but successful, hibernation. People are coming back to nuclear energy as they did back in the seventies and early eighties, and the fusion community is gearing up to show the world that, finally and for the benefit of all humanity, the power inside the sun, can be put to work safely here on earth.

The road to harnessing the process that fuels the stars in a controlled fashion, and thus providing humanity with a virtually endless and cheap source of energy has been a long and winding one. The path to controlled thermonuclear fusion has taken many twists and turns during more than fifty years of continuous research; 
from Bennett's pinch and the original stellarator of Spitzer, to the currently under construction mega-scale experimental demos like the ITER $^{1}$ and the National Ignition Facility (NIF).

During all these years the scientific community has worked hard in different fronts; some are trying to understand, and ultimately solve, the problems of nuclear and plasma physics; others are busy dealing with the engineering and more practical aspects of the implementation. The field known as fusion technology deals with applied research; it lies in between physics and engineering, bringing together the basic physical principles and engineering tools to address a specific issue. The present work in neutron imaging is a typical example of this kind of research.

\subsection{Nuclear Fusion Fundamentals}

Nuclear fusion is the process through which light nuclei combine forming a heavier nucleus, light particles and energy. The energy is released as kinetic energy of the products and it is due to the difference in masses between reagents and products. In this sense the fusion reaction is exothermic or exoergic. The $Q$ value of a nuclear reaction is given by[1]:

$$
Q=\left(m_{\text {initial }}-m_{\text {final }}\right) c^{2}
$$

\footnotetext{
${ }^{1}$ Formerly an acronym for International Thermonuclear Experimental Reactor, it also means journey or path in Latin.
} 
In order to obtain a $Q>0$, the products must have lower mass than the light reagents. The fact that the products have lower mass is explained introducing the concept of binding energy $B$, which is the difference in $\operatorname{mass}^{2}$ between a nucleus of mass $M$ and its constituent $N$ neutrons and $Z$ protons

$$
B=\left\{Z m_{p}+N m_{n}-M\right\}
$$

where $m_{p}$ and $m_{n}$ are the proton and neutron masses respectively. Using the semiempirical mass formula given by[2][3]:

$$
M=N m_{n}+Z m_{p}-a_{\nu} A+a_{s} A^{2 / 3}+a_{c} \frac{Z(Z-1)}{A^{1 / 3}}+a_{a} \frac{(N-Z)^{2}}{A}+\frac{a_{p} \delta}{A^{3 / 4}}
$$

one can calculate the binding energy per nucleon $(B / A)$

$$
\frac{B}{A}=a_{\nu} A-\frac{a_{s}}{A^{1 / 3}}-a_{c} \frac{Z(Z-1)}{A^{4 / 3}}-a_{a} \frac{(N-Z)^{2}}{A^{2}}-\frac{a_{p} \delta}{A^{7 / 4}}
$$

Figure 1.1 shows a plot of $B / A$ as a function of atomic number $A$. Notice how by combining light nuclei into heavier ones, i.e., more tightly bound nuclei, more binding energy is available for release. Fusion occurs in nature all the way from hydrogen up to around $A=60$, the isotope with the highest binding energy per nucleon. Above this point energy is released if the nuclei split, as it occurs during fission. Heavier isotopes can be formed through fusion processes but at extreme conditions, like those encountered during supernovae.

Fusion does not spontaneously occur under normal temperature and pressure conditions. The positively charged nuclei must overcome the repulsive Coulomb barrier

\footnotetext{
${ }^{2}$ In nuclear physics is common practice to express the masses as energey equivalent, in units of $\mathrm{MeV}$. This is done using Einstein's mass-energy relation $E=m c^{2}$.
} 


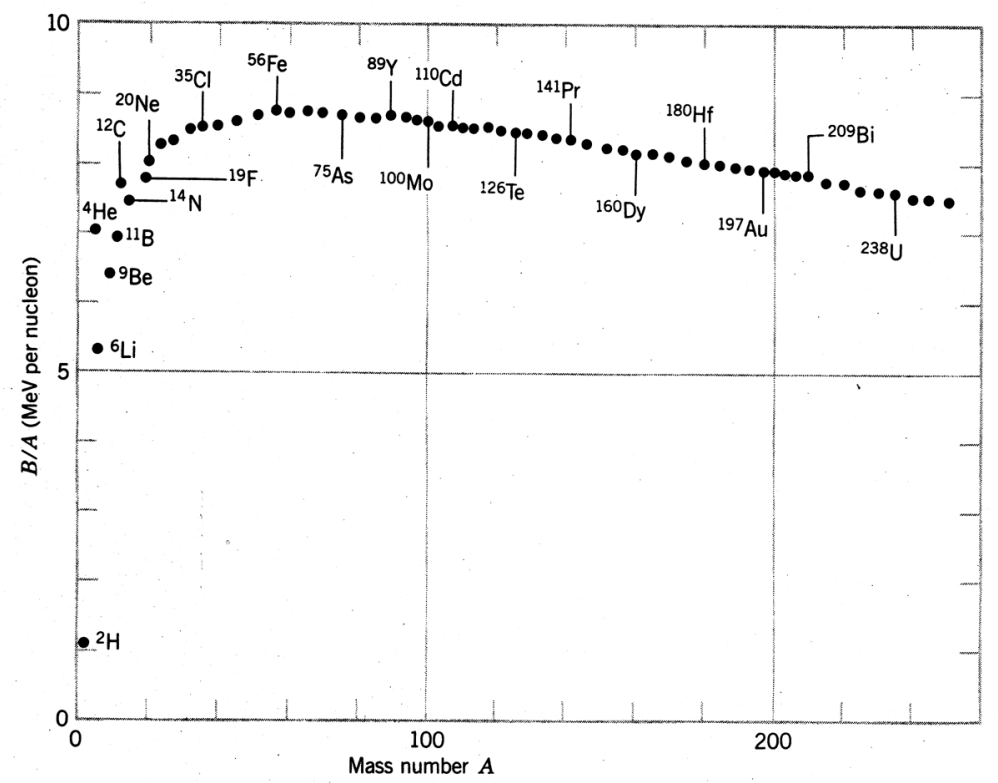

Figure 1.1: Binding energy per nucleon as a funtion of mass number. Notice the maximum around $A=60$. Taken from Kenneth S. Krane, Introductory Nuclear Physics. John Wiley \& Sons 1988

in order to fuse. The Coulomb potential is given by[4]

$$
U(r)=\frac{1}{4 \pi \epsilon_{o}} \frac{q_{a} q_{b}}{r}
$$

where $q_{a}$ and $q_{b}$ are the charges of the two nuclei and $r$ the distance between them. The potential energy at $r=R_{o}=R_{a}+R_{b}$, where $R_{a}$ and $R_{b}$ are the equivalent niclei radii, is called the Coulomb Barrier, and as can be seen in figure 1.2, it is the point of maximum energy before the attractive nuclear forces take over. As an example, the Coulomb barrier for two protons is of the order of $700 \mathrm{keV}$, which is equivalent to $8.12 \times 10^{9} \mathrm{~K}^{3}$ This is extremely high, orders of magnitude higher than the temperature

\footnotetext{
${ }^{3}$ One eV (electron volt) is the energy an electron acquires while moving through a $1 \mathrm{~V}$ potential, $1 \mathrm{eV}=1.602 \times 10^{-19} \mathrm{~J}$. It is also used as a unit of temperature, in the sense of thermal energy, through $E=k T$ where $k$ is Boltzmann's constant and $T$ is in $\mathrm{K}, 1 \mathrm{eV}=11600 \mathrm{~K}$.
} 


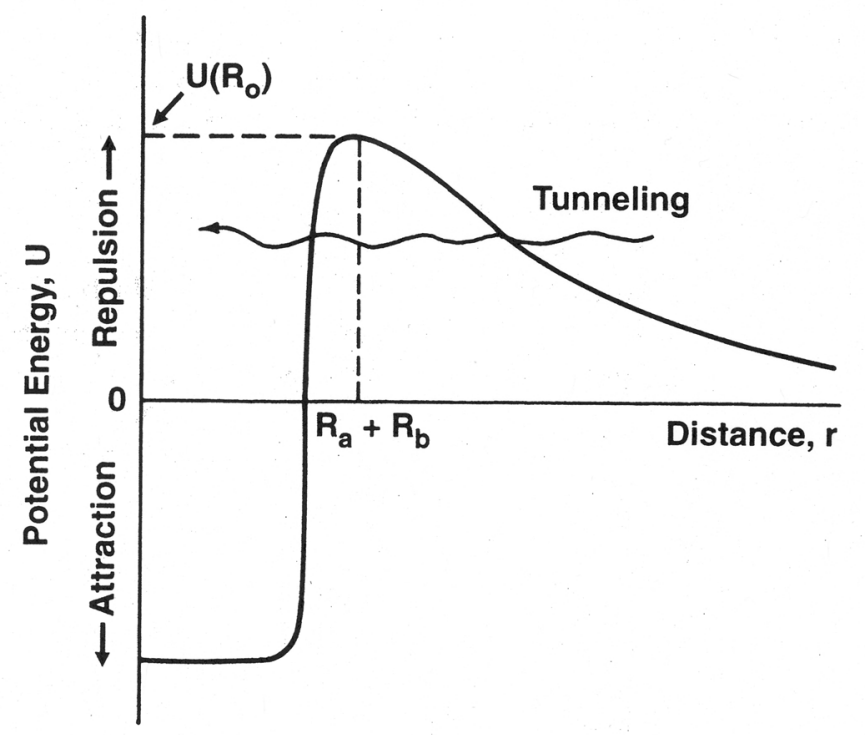

Figure 1.2: Diagram of the potential that exists between two positively charged ions. At distance $r=R_{o}=R_{a}+R_{b}$ the repulsion is maximum (Coulomb Barrier), at shorter distances the atractive nuclear forces take over keeping the ions together. Taken from A. A. Harms et.al. Principles of Fusion Energy. World Sceintific. Singapore, 2000.

in the interior of the sun $\left(10^{6} \mathrm{~K}\right)$. The explanation of why fusion can occur at lower temperatures than the ones dictated by the electrostatic repulsion alone, is given by the quantum mechanical effect called tunneling, i.e., the proton wave function has a small, though finite, probability beyond the cusp of the Coulomb potential, allowing the proton to penetrate the barrier and fuse. The tunneling probability is given by[4]:

$$
\operatorname{Pr} \propto \frac{1}{v_{r}} \exp \left(-\gamma \frac{q_{a} q_{b}}{v_{r}}\right)
$$

where $v_{r}$ is the relative speed of the moving particles and $\gamma$ is a constant.

$$
U(r)=\frac{1}{4 \pi \epsilon_{o}} \frac{q_{a} q_{b}}{\left(R_{a}+R_{b}\right)}
$$

In the first half of the twentieth century it was determined that stars are fueled 
by the fusion of hydrogen into helium. The most abundant of the fusion processes that take place in the sun is the so called $\mathrm{p}-\mathrm{p}$ process, it is given by:[1]:

$$
\begin{aligned}
{ }^{1} \mathrm{H}+{ }^{1} \mathrm{H} & \longrightarrow{ }^{2} \mathrm{H}+e^{+}+\nu+1.44 \mathrm{MeV} \\
{ }^{2} \mathrm{H}+{ }^{1} \mathrm{H} & \longrightarrow{ }^{3} \mathrm{He}+\gamma+5.49 \mathrm{MeV} \\
{ }^{2} \mathrm{H}+{ }^{1} \mathrm{H} & \longrightarrow{ }^{3} \mathrm{He}+\gamma+5.49 \mathrm{MeV} \\
{ }^{3} \mathrm{He}+{ }^{3} \mathrm{He} & \longrightarrow{ }^{4} \mathrm{He}+{ }^{1} \mathrm{H}+{ }^{1} \mathrm{H}+\gamma+12.86 \mathrm{MeV}
\end{aligned}
$$

Accounting for the electrons required for atom neutrality and the ones annihilated with the positrons, the net reaction converts four hydrogen atoms into one ${ }^{4} \mathrm{He}$ and releases energy acording to

$$
4{ }^{1} \mathrm{H} \longrightarrow{ }^{4} \mathrm{He}+26.7 \mathrm{MeV}
$$

Proton - proton fusion is very hard to achieve outside the sun. On earth conditions like the ones prevalent at the sun's core are not available. It is necessary to consider other reactions that work at lower temperatures. Some of the candidate reactions involving light isotopes $\operatorname{are}^{4}[5]$ :

$$
\begin{aligned}
& \mathrm{D}+\mathrm{T} \longrightarrow{ }^{4} \mathrm{He}+n+17.59 \mathrm{MeV} \\
& \mathrm{D}+\mathrm{D} \longrightarrow{ }^{3} \mathrm{He}+n+3.27 \mathrm{MeV} \\
& \mathrm{D}+\mathrm{D} \longrightarrow \mathrm{T}+p+4.03 \mathrm{MeV} \\
& \mathrm{T}+\mathrm{T} \longrightarrow n+n+{ }^{4} \mathrm{He}+11.3 \mathrm{MeV}
\end{aligned}
$$

\footnotetext{
${ }^{4}$ Note the use of $\mathrm{D}$, deuterium, and $\mathrm{T}$, tritium, instead of ${ }^{2} \mathrm{H}$ and ${ }^{3} \mathrm{H}$ respectively.
} 
As an example, the Coulomb barrier for two deuterons (DD) is around $400 \mathrm{keV}[4]$ which is equivalent to $4.64 \times 10^{9} \mathrm{~K}$; lower than proton-proton, but still high.

The best reaction for energy production is not just the one with the highest energy release; it is necessary to consider the reaction rate as well, that is, the number of particles produced per unit time for a specific reagent concentration and energy. The reaction rate can be written as:

$$
R=N_{a} N_{b}\langle\sigma v\rangle_{a b}
$$

where $N_{a}$ and $N_{b}$ are the particle densities of the two reacting species and $\langle\sigma v\rangle_{a b}$ is the so called 'sigma-vee' parameter or reactivity. It represents the average of the reaction cross section $\sigma_{a b}$, at all velocities present in a given volume

$$
\langle\sigma v\rangle_{a b}=\int_{\mathbf{v}_{a}} \int_{\mathbf{v}_{b}} \sigma_{a b}\left(\left|\mathbf{v}_{a}-\mathbf{v}_{b}\right|\right)\left|\mathbf{v}_{a}-\mathbf{v}_{b}\right| F_{a}\left(\mathbf{v}_{a}\right) F_{b}\left(\mathbf{v}_{b}\right) d^{3} \mathbf{v}_{a} d^{3} \mathbf{v}_{b}
$$

where $\mathbf{v}_{a}$ and $\mathbf{v}_{b}$ are velocity vectors in three-dimensional space and $F_{a}\left(\mathbf{v}_{a}\right)$ and $F_{b}\left(\mathbf{v}_{b}\right)$ are the probability distributions. For most fusion scenarios the plasma is assumed to obey a Maxwellian distribution

$$
F(\mathbf{v})=\left(\frac{m}{2 \pi k T}\right)^{3 / 2} \exp \left(\frac{-m \mathbf{v}^{2}}{2 k T}\right)
$$

where $m$ is the mass of the particle, $\mathrm{k}$ is Bolztmann's constant and $T$ is the temperature. Tabulated values of $\langle\sigma v\rangle_{a b}$ as a function of temperature are available in the technical literature[6][7][8]; figure 1.3 shows the reactivity for different reactions as a function of the average temperature. The label 'thermonuclear fusion' arises from the 


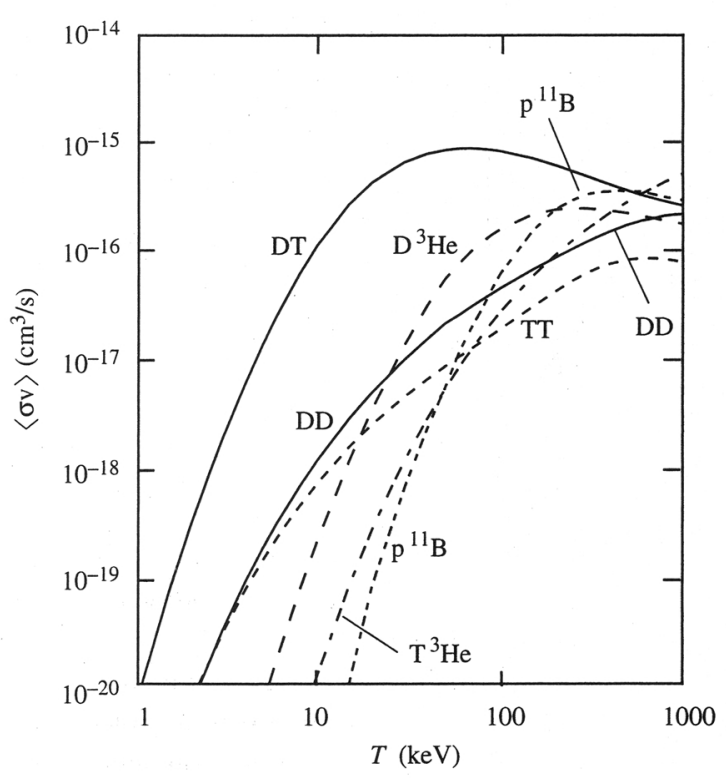

Figure 1.3: $\langle\sigma v\rangle_{a b}$ as a function of average temperature for differnet fusion reactions. Notice how DT has the highest values of reactivity for this temperature range. Taken from S. Atzeni and J. Meyer-ter-Vehn. The Physics of Inertial Fusion. Oxford University Press. Oxford, 2004.

fact that all the particles involved in the reaction have a certain kinetic temperature $(k T)$, as opposed to the case of an ion beam impinging on a static target, where the kinetic energy is that of the incoming ions.

Based only on the $\langle\sigma v\rangle$ parameter, the DT reaction is the best candidate for an energy producing operation; it has the highest reactivity at achievable temperatures. Unfortunately not everything is perfect with DT fusion: almost $80 \%$ of the energy produced is carried away by the neutron, and recovering this energy is a major fusion technology challenge. It would be ideal, from the energy recovery point of view, to use reactions that only produce charged particles, so a direct energy conversion approach could be used $[9]$; the problem with these reactions is the high temperatures required 
to obtain high reactivities. Some of these aneutronic reactions are listed bellow; they are based in ${ }^{3} \mathrm{He},{ }^{6} \mathrm{Li}$ and ${ }^{11} \mathrm{~B}[5]$.

$$
\begin{aligned}
{ }^{3} \mathrm{He}+{ }^{3} \mathrm{He} & \longrightarrow 2 p+{ }^{4} \mathrm{He}+12.86 \mathrm{MeV} \\
\mathrm{D}+{ }^{3} \mathrm{He} & \longrightarrow{ }^{4} \mathrm{He}+p+18.3 \mathrm{MeV} \\
p+{ }^{6} \mathrm{Li} & \longrightarrow{ }^{3} \mathrm{He}+{ }^{4} \mathrm{He}+4.02 \mathrm{MeV} \\
{ }^{3} \mathrm{He}+{ }^{6} \mathrm{Li} & \longrightarrow p+2{ }^{4} \mathrm{He}+1.69 \mathrm{MeV} \\
p+{ }^{11} \mathrm{~B} & \longrightarrow{ }^{4} \mathrm{He}+8.68 \mathrm{MeV} \\
p+{ }^{11} \mathrm{~B} & \longrightarrow{ }^{12} \mathrm{C}+\gamma+15.96 \mathrm{MeV}
\end{aligned}
$$

For now DT fusion is the best candidate for commercial energy production; and it may lead to future advanced fuel cycles that enjoy the benefits of the harder to achieve reactions.

The two main processes necessary to attain controlled fusion are: heating the fuel to the required temperatures, so the reaction rate is high enough, and confining it for a sufficient long time, so a net gain in energy is achieved. Assuming an equimolar mixture of deuterium and tritium with particle density n, equation 1.8 becomes

$$
R=\frac{n^{2}}{4}\langle\sigma v\rangle_{D T}
$$

The energy released, E, in a fusion device is given by

$$
E=R \tau Q=\frac{n^{2}}{4}\langle\sigma v\rangle_{D T} \tau Q
$$

where $\mathrm{Q}$ is the amount of energy released per fusion event (17.6 MeV for DT) and $\tau$ 
is the duration of the experiment. In order to generate a net energy gain, E has to be greater than the kinetic energy of all the particles in the plasma; using the assumed Maxwellian distribution and accounting for both ions and electrons, this is can be written as

$$
\begin{gathered}
\frac{n^{2}}{4}\langle\sigma v\rangle_{D T} \tau Q>2 \frac{3}{2} n k T \\
n \tau>\frac{12 k T}{\langle\sigma v\rangle_{D T} Q}
\end{gathered}
$$

Equation 1.14 is a version of the so-called Lawson Criterion[10], it expresses the density and confinement time necessary to achieve a net gain using a fusion reaction at a given temperature. For the particular case of a DT plasma at $10 \mathrm{keV}$, the minimum $n \tau$ is $10^{15} \mathrm{~s} \mathrm{~cm}^{-3}$. The higher the particle density, the shorter the required confinement time and vice versa; this fact has been exploited in designing the two main confinement schemes described bellow.

In magnetic confinement fusion (MCF) a plasma is generated and heated using intense electrical currents, neutral beams and RF sources, and confined into a specific geometry by very strong magnetic fields. Among the most promising confinement geometries are the Tokamak ${ }^{5}$ and the Spheromak. ITER, currently under construction in France, is a Tokamak. The typical particle density of magnetic plasmas is $10^{14} \mathrm{~cm}^{-3}$, which implies a confinement time of $10 \mathrm{~s}$.

In inertial confinement fusion (ICF) the temperature and pressure necessary for the fusion reaction are generated when a high intensity energy pulse compresses a

\footnotetext{
${ }^{5}$ From the Russian tokamák, acronym from toroidál'naya kámera s aksiál'nym magnítnym pólem, which translates into toroidal chamber with an axial magnetic field.
} 
capsule containing a very small amount of fuel, and it is held together by the mass inertia. The energy pulse is delivered to the capsule in the form of a laser light, x-rays or heavy ions. In ICF the typical particle density is higher than solid density, around $10^{26} \mathrm{~cm}^{-3}$, the confinement time is of the order of $10^{-11} \mathrm{~s}$. The NIF uses a powerful laser to implode a spherical shell full of DT fuel.

Since the objective of the present work is to study neutron imaging at the NIF, all attention will be directed to $\mathrm{ICF}$. The reader wishing more information about MCF is directed to texts in that area.

\subsection{Inertial Confinement Fusion Concepts}

In ICF the required high temperatures are achieved though the very fast compression of the DT fuel mixture. The required pressures are so high (in the Giga bar range) that they can not be achieved by mechanical means like a hydraulic press. Higher pressures can be generated with the use of chemical explosives, this approach has been used, since the Manhattan project, in nuclear weapons. For ICF to work in a laboratory or power plant, a safer and more efficient form of pressure generation has to be used. The solution is the use of ablation implosions. Ablation is the process through which a material heats up, ionizes and vaporizes after a burst of energy is applied to it. This material vaporization produces a force, due to momentum conservation, in the solid material, compressing it. This process can be illustrated with the derivation of the classic rocket equation, which relates the change in speed of the 
ship to its change in mass. For a rocket of instantaneous mass $M(t)$, moving at a velocity $v(t)$, with the propellant escaping in the oposite direction at constant speed $U$, both with respect to and inertial frame, and in the absence of gravity and drag, the conservation of momentum requires that

$$
\begin{array}{r}
\frac{\mathrm{d}(M v)}{\mathrm{d} t}+\frac{\mathrm{d}(M U)}{\mathrm{d} t}=0 \\
M \frac{\mathrm{d} v}{\mathrm{~d} t}+v \frac{\mathrm{d} M}{\mathrm{~d} t}=-U \frac{\mathrm{d} M}{\mathrm{~d} t}
\end{array}
$$

introducing $v_{e x}=U+v$, the exhaust velocity relative to the rocket

$$
M \frac{\mathrm{d} v}{\mathrm{~d} t}=-v_{e x} \frac{\mathrm{d} M}{\mathrm{~d} t}
$$

Working in a small time interval and integrating between the initial $\left(m_{o}\right)$ and the final masses $\left(m_{f}\right)$ gives

$$
v=v_{e x} \ln \left(\frac{m_{o}}{m_{f}}\right)
$$

For an ICF target the same result is valid, but $v_{e x}$ is given by[11] $v_{e x}=P_{a} / \dot{m}$, where $\dot{m}$ is the mass ablation rate and $P_{a}$ is the ablation pressure, both of which are related to the incident light flux.

Equation 1.18 does not distinguish between a solid sphere and a shell; it can be shown[3] that higher implosion speeds are achieved by using a thin shell

$$
\frac{v_{\text {shell }}}{v_{\text {solid }}} \propto\left(\frac{R}{\Delta R}\right)^{1 / 2}
$$

where $R$ is the initial radius and $\Delta R$ the thickness of the shell.

It is known from hydrodynamics that isentropic compression is more efficient than simple shock compression. High density materials generate less entropy when 


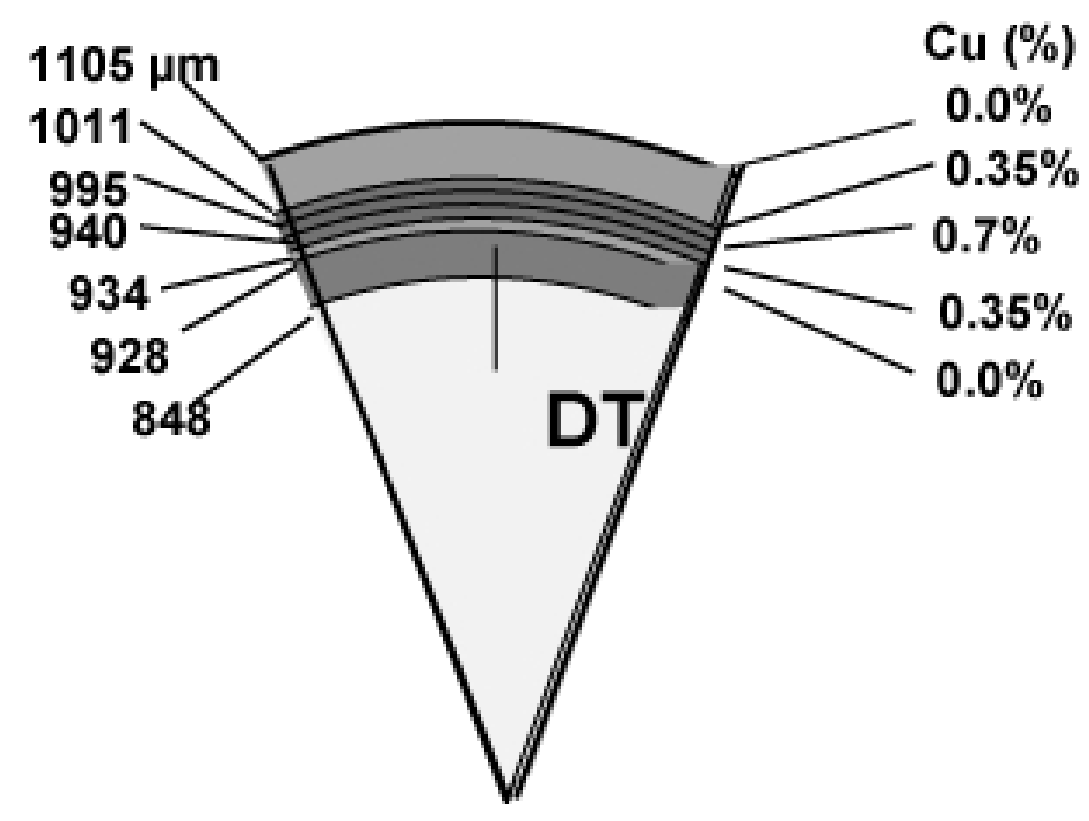

Figure 1.4: Typical ICF capsule geometry. The shell is made of $\mathrm{Cu}$ dopped plastic, notice the graded profile. The DT forms an ice layer in equilibrium with a vapor phase in the center.

undergoing compression; for this reason in an ICF target designed for high gain the fuel is solid DT, which requires temperatures bellow the triple point, i.e., 19.7 K. At this temperature the fuel forms a thin ice layer inside the shell, and fills the center portion with a vapor in equilibrium. Figure 1.4 shows the structure a typical ICF target. Another consequence of this configuration is the formation of the so-called 'hot spot' at the center of the compressed capsule. It is this region of high temperature and low density which sparks the thermonuclear burn that propagates through the colder high density fuel that surrounds it. This approach is more efficient energetically than trying to heat all the fuel inside the capsule. One of the most important parameters used in ICF is the $\rho R$ or 'areal density' which is a measure of how compressed is the 
fuel, both in number of particles per unit volume and in space (size). It can be shown that the fuel burn fraction is related to $\rho R$ by[12]

$$
f_{b}=\frac{\rho R}{\beta(T)+\rho R}
$$

where $\beta(T)$ is the burn parameter, which has a value of 6 to $9 \mathrm{~g} / \mathrm{cm}^{2}$ in the 20 to 40 keV range. A $30 \%$ burn fraction requires $\rho R=3 \mathrm{~g} / \mathrm{cm}^{2}$.

$\rho R$ can also be used to show the need for compression in ICF. Assuming a uniform density target with $\rho=0.225 \mathrm{~g} / \mathrm{cm}^{3}$ (DT liquid density), and using the previously found $\rho R=3 \mathrm{~g} / \mathrm{cm}^{2}$, the target mass is given by

$$
\begin{aligned}
M & =\frac{4}{3} \pi \rho R^{3} \\
M & =\frac{4}{3} \pi(\rho R)^{3} \frac{1}{\rho^{2}} \\
M & =2234 \mathrm{~g}
\end{aligned}
$$

The specific yield of equimolar DT can be calculated as

$$
\begin{gathered}
y_{\mathrm{DT}}=17.6 \frac{\mathrm{MeV}}{\mathrm{DT}} \times 6.02 \times 10^{23} \frac{\mathrm{DT}}{\mathrm{mol} \mathrm{DT}} \times \frac{1 \mathrm{~mol} \mathrm{DT}}{5} \times 1.6 \times 10^{-13} \frac{\mathrm{J}}{\mathrm{g} \mathrm{DT}} \times 1.9 \\
y_{\mathrm{DT}}=3.39 \times 10^{11} \frac{\mathrm{J}}{\mathrm{g} \mathrm{DT}}
\end{gathered}
$$

Assuming a burn fraction of $30 \%$, the uncompressed fuel will have a yield of $2.272 \times$ $10^{14} \mathrm{~J}$, which is equivalent to 54.3 kilotons of $\mathrm{TNT}^{6}$. This energy release is extremely high. If instead a very high density is used, say 1250 times liquid density, the required mass is just $1.43 \mathrm{mg}$ and the energy released is $1.454 \times 10^{8} \mathrm{~J}$, which is $34.75 \mathrm{~kg}$ TNT.

\footnotetext{
${ }^{6}$ The 'TNT equivalent' was defined in order to quantify the energy released in an explosion. One kilogram of TNT releases, by definition, $4.184 \times 10^{6} \mathrm{~J}$.
} 
Although this may seem high, it is important to remember that it is the momentum and not just the energy released, which can cause structural damage. The mass involved is so small that its momentum is very low.

Another important parameter for ICF calculations is the confinement time, $\tau_{c}$. This is the time the compressed fuel will hold together before it blows apart due to the increasing internal pressure. In a first-order approximation $\tau_{c}$ can be calculated as

$$
\tau_{c}=\frac{R}{C_{s}}
$$

which is the time it will take a shock wave, moving at the speed of sound $\left(C_{s}\right)$, to travel from the center to the surface of the compressed sphere. A more detailed calculation that accounts for the actual fuel distribution by using the mass averaged time gives[12]

$$
\tau_{c}=\frac{R}{4 C_{s}}
$$

The speed of sound in a plasma is given by[13]

$$
C_{s}=\left(\frac{Z \gamma k T_{e}+\gamma k T_{i}}{m_{i}}\right)^{1 / 2}
$$

For a mono atomic gas like hydrogen $\gamma=5 / 3$ and taking $m_{i}=2.5 m_{p}$ as the average molecular weight for an equimolar DT mixture, and $T_{e}=T_{i}=T$ equation 1.24 reduces to

$$
C_{s}=11301(T(\mathrm{eV}))^{1 / 2} \frac{\mathrm{m}}{\mathrm{s}}
$$




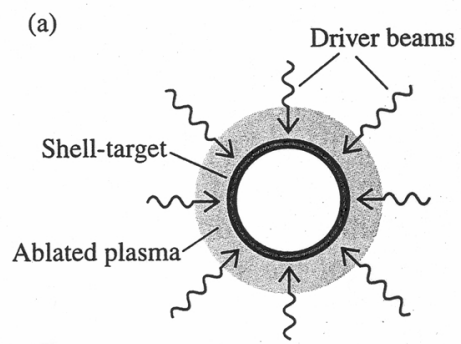

(b)
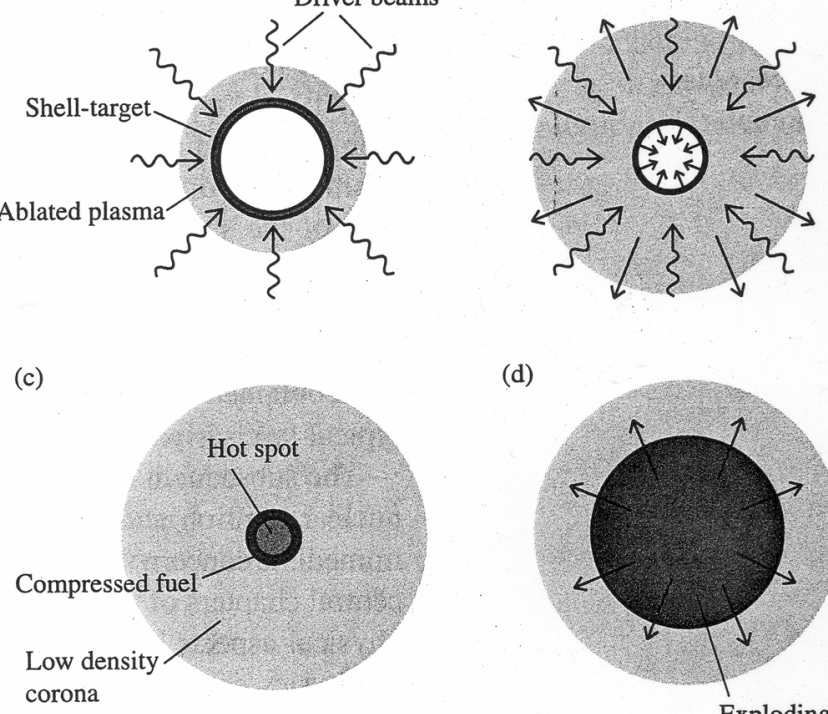

(d)

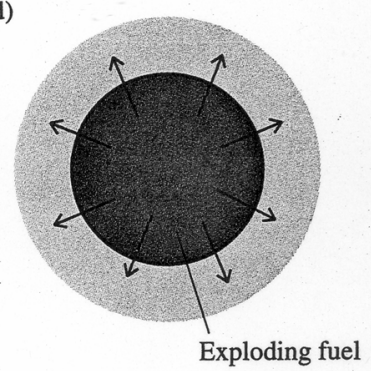

Figure 1.5: ICF sequence: a) Irradiation b) Implosion by ablation c) Central ignition and d) Burn and explosion. Taken from S. Atzeni and J. Meyer-ter-Vehn. The Physics of Inertial Fusion. Oxford University Press. Oxford, 2004.

A typical ICF capsule has a final radius of 100 microns and burns at $20 \mathrm{keV}$, which gives a confinement time of 62.5 pico seconds.

The four basic ICF steps: irradiation, implosion by ablation, central ignition, and burn and explosion, are summarized in figure 1.5.

There are two main ways of delivering the energy to the capsule: directly and indirectly. In the direct drive approach the intense laser pulse or ions impinge directly on the surface of the capsule. In indirect drive, there is an intermediate step that converts the laser into thermal x rays, and these in turn impinge on the capsule. Both approaches have their advantages and are being pursued in parallel by the scientific community. The Lawrence Livermore National Laboratory (LLNL) ICF program is 
devoted to the development of indirect drive, while the Laboratory for Laser energetics (LLE) at the University of Rochester is pursuing direct drive.

The conversion of the laser into $\mathrm{x}$ rays is achieved by the use of a hohlraum ${ }^{7}$, that is, a cylindrical shell made of high Z materials, usually gold and uranium, that surrounds the target. The laser heats the inner wall of the hohlraum producing thermal $\mathrm{x}$ rays. The typical radiation temperatures are in the 100 to $300 \mathrm{eV}$ range. The main advantages of indirect drive are: smoother heating of the capsule surface, lower hot electron generation and higher implosion velocities. The biggest disadvantage is the very low coupling efficiency, 10\%, compared to $80 \%$ of direct drive. The current hohlraum design for NIF is illustrated in figure 1.6. The laser pulse used in indirect drive, shown in figure 1.7, has a very specific profile that increases in power in several successive steps. As the power increases so does the hohlraum temperature. This laser pulse shape minimizes the entropy generated in the capsule.

\subsection{The National Ignition Facility}

ICF research has been going on around the world since the 1950s, first as an extension of nuclear weapons development, and then in its own right. One of the first scientists to suggest and document the use of lasers to achieve the conditions necessary for nuclear fusion was laser pioneer and nobelist Nicolay Gennadiyevich Basov[14][15]. In the United States work on laser-plasma interaction, begun at the

\footnotetext{
${ }^{7}$ From the German hohlraum, which means hollow space.
} 


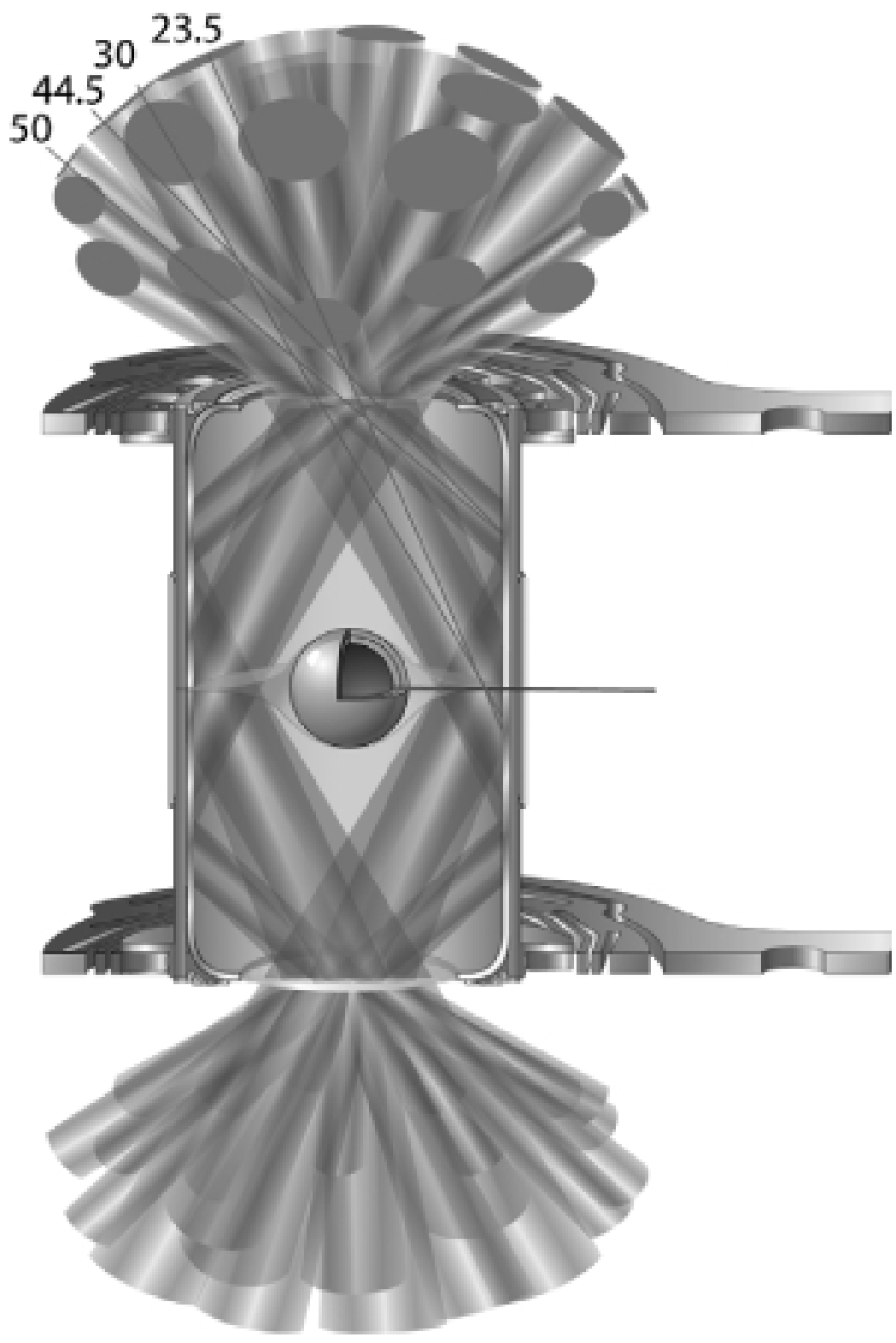

Figure 1.6: NIF Hohlraum geometry. This schematic shows the fuel capsule inside the hohlraum and some of the 192 laser beams entering from the top and bottom through the laser entrance holes (LEHs) illuminating four rings on the inner wall. The capsule, initially under vacuum, is filled with DT gas through a two micron diameter fill tube, partially shown extending to the right, then the temperature is lowered and kept bellow $19.7 \mathrm{~K}$ by a cryostat, of which only the so-called 'cold fingers' are shown. 


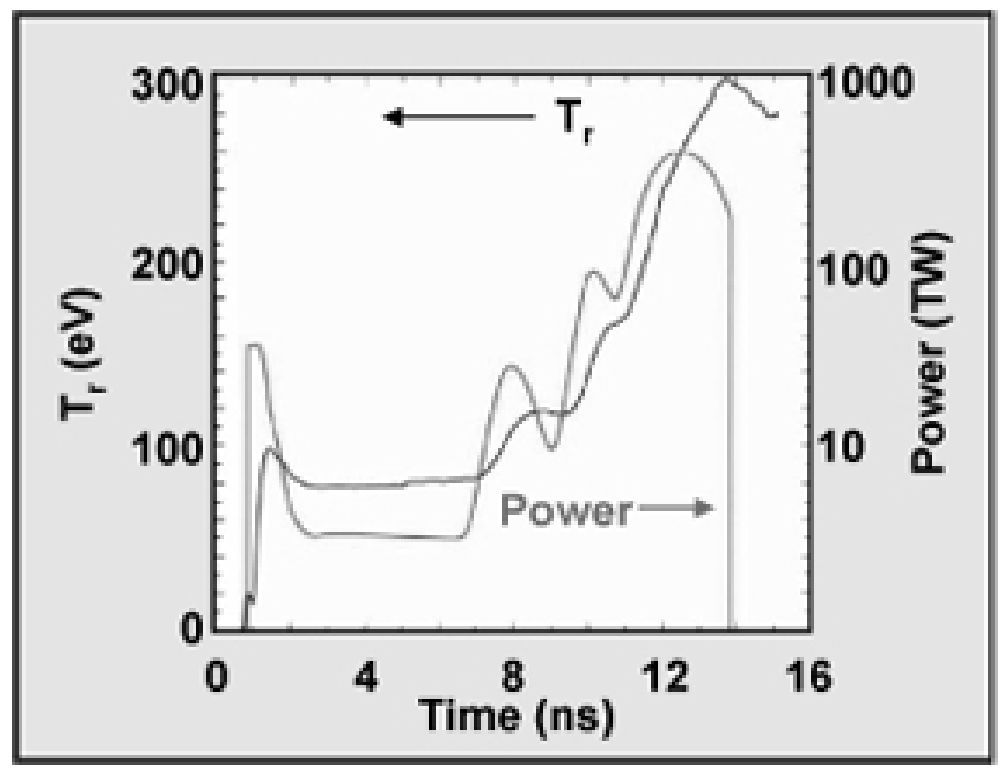

Figure 1.7: Typical NIF laser pulse and radiation temperature inside the hohlraum.

Lawrence Livermore National Laboratory (LLNL) in 1962, led to the seminal paper by Nuckolls et al.[16] in 1972 which dealt with direct drive implosions of shells of DT. By 1975 LLNL had shifted all research efforts to indirect drive implosions. The first indirect drive implosions occurred in 1976 at the Cyclops laser[17] (One beam, $100 \mathrm{~J}$ at 1.06 microns) producing $2 \times 10^{5}$ neutrons. Subsequent facilities were Argus[18] (Two beams, 1 - $2 \mathrm{~kJ}$ at 1.06 microns) and Shiva[19] (20 beams, $20 \mathrm{~kJ}$ at 1.06 microns). In 1981 scientists at the University of Rochester demonstrated the high-efficiency frequency conversion of laser light using potassium dihydrogen phosphate (KDP) crystals[20][21]. This opened the door to the use of 0.53 and 0.35 micron light, which had been predicted to have higher coupling efficiency than the 1.06 micron laser. The next ICF facility was Nova[22] (Ten beams, $40 \mathrm{~kJ}$ at 0.53 and 0.35 microns), 
which operated from 1985 to 1999. After the initial success of Nova the Department of Energy (DOE) proposed a 5 - 10 MJ laser labeled the Laboratory Micro Fusion Facility, with proposed yields of 200 - 1000 MJ. This facility proved to be a very big step forward in research and development, and a more conservative intermediate step was suggested. The result was the NIF[23][24][25], which was approved by DOE in 1993 and began construction in 1995.

The NIF, shown in figure 1.8, will deliver 1.8 MJ of ultraviolet (0.35 microns) laser light using 192 beams, these will enter the hohlraum from the top and the bottom ${ }^{8}$. A typical beam line is shown in figure 1.9. The laser pulse is born and shaped in the master oscillator where its energy is in the nanojules. The final high energy is achieved in the flashlamp-pumped multi pass glass amplifiers. The final optics that surround the 10-m target chamber, change the wavelength to 0.35 microns and focus the beams to 500 microns spots. All the LLNL lasers have been based on the neodymium doped glass (Nd:glass) gain medium. This laser scheme has been widely studied and the scientific community decided to use it at NIF because of the long experience with it. This laser shows several useful lasing transitions (1.35, 1.06 and 0.914 microns) but the most frequently used is the 1.06 microns in conjunction with frequency doublers or triplers. Figure 1.10 shows a collection of the glass used in the lasers at LLNL.

The activities required to perform a successful ignition attempt at NIF are collected in the so-called National Ignition Campaign (NIC). In particular it introduces

\footnotetext{
${ }^{8}$ Provisions have been made to modify the beam layout and allow for uniform target illumination for direct drive. These experiments are planed after the initial indirect drive ignition attempts.
} 


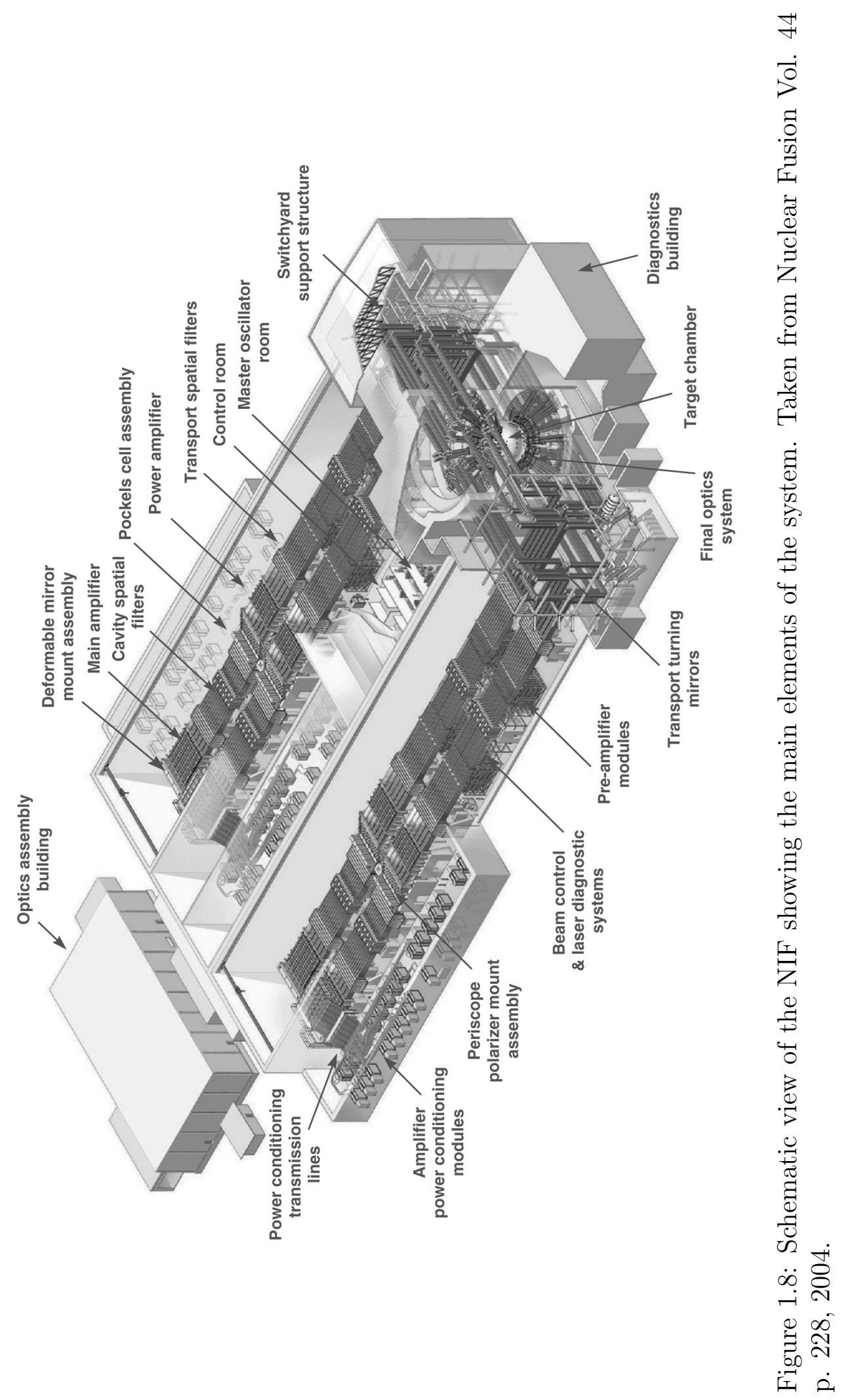




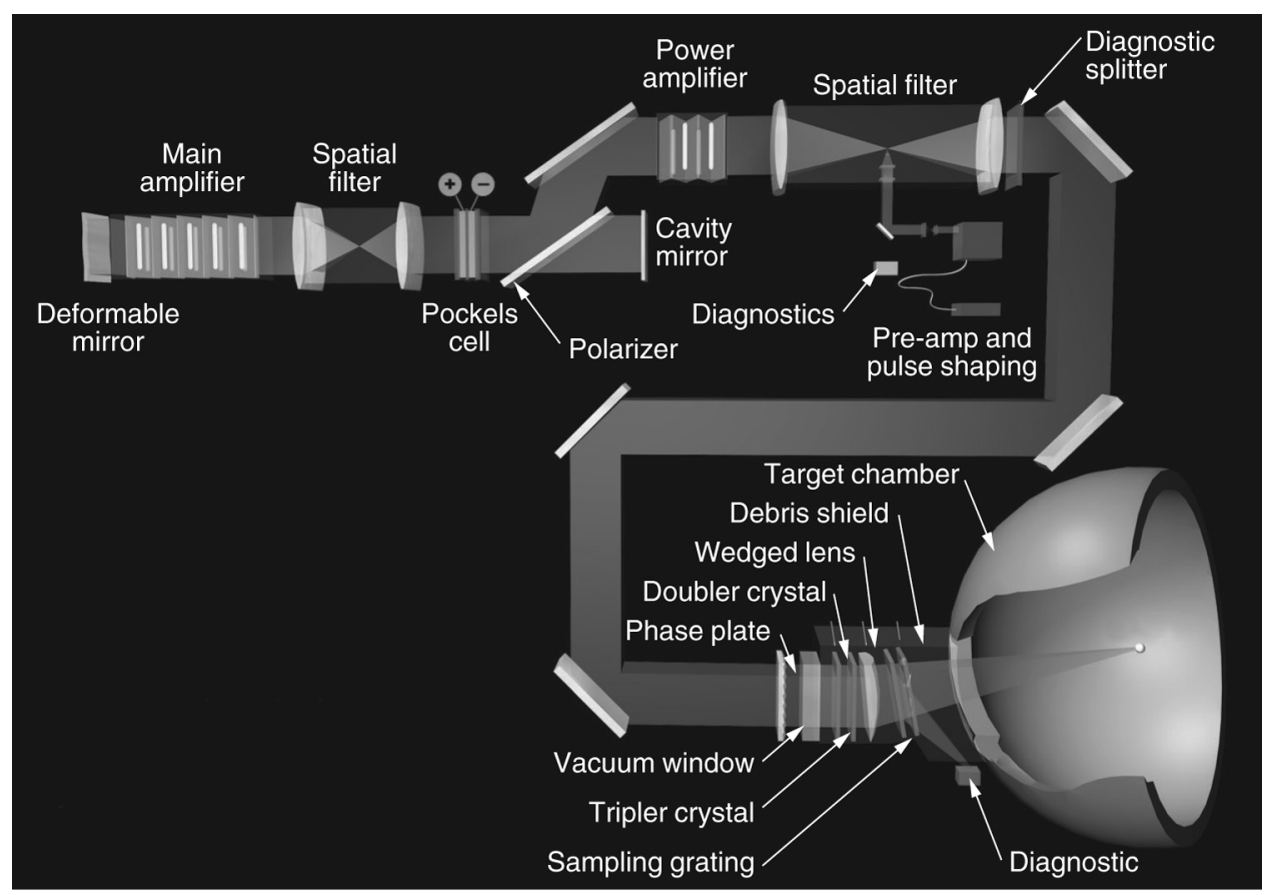

Figure 1.9: The layout of NIF's major beamline components through which a pulse of laser light travels from injection to final focus on the target. Taken from Science and Technology Review September LLNL, 2003.

a series of tuning campaigns that will optimize the main key and target parameters necessary for ignition. The tuning campaigns are: hohlraum energetics, hohlraum symmetry, shock timing, laser pointing and capsule hydrodynamics. Once the parameters have been set, the actual ignition experiments with DT capsules will begin, culminating with full energy attempts with cryogenic high yield targets, which are expected to achieve a gain of around 10 .

The variables that will be measured during the NIC are: primary yield, fuel $\rho R$, ion temperature, fusion reaction rate and spatial distribution of the fuel. Some of these variables will be measured by multiple devices, providing redundancy and cross 


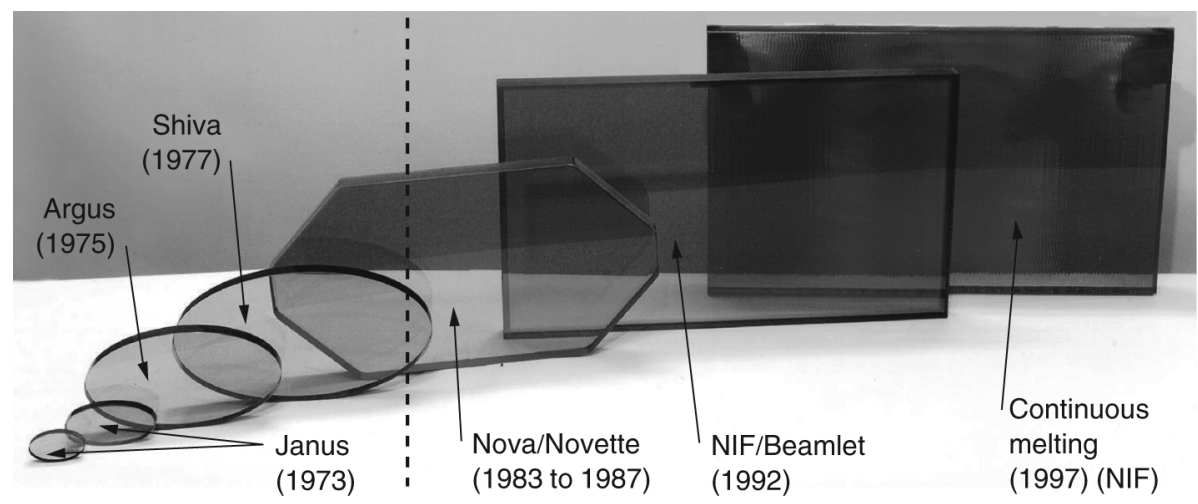

Figure 1.10: Collection of laser glass used in the laser systems at LLNL. The increase in size has been necessary in order to accomodate higher and higher intensities and not damage the glass. The vertical dashed line between Janus and Nova represents the change in manufacturing technique from silicate to phosphate. Taken from Science and Technology Review September LLNL, 2003.

reference. The official ignition target diagnostics are[26]:

1. Protex[27]: absolute DT neutron yield. The measurement is based on the recoil protons produced when the $14 \mathrm{MeV}$ neutrons pass through a thin plastic foil. The absolute sensitivity is achievable due to the well known $(n, p)$ elastic scattering cross section and the geometry of the detector itself.

2. Neutron Time-of-Flight (nTOF): neutron yield and ion temperature. This diagnostic is based on current-mode detectors (fast scintillators and photomultiplier tubes (PMTs)) placed at large distances from the source. The signal from the PMTs is recorded by high bandwidth oscilloscopes. The neutron yield is obtained from the recorded pulse shape (previous calibration). The ion temperature can be found by looking at the broadening of the $14 \mathrm{MeV}$ neutron peak. 
3. Neutron Bang Time/Reaction History Diagnostic (NBT/RHD): Bang time refers to the time interval between the beginning of the laser pulse and the maximum of fusion neutron or gamma emission. The RHD follows the fusion reaction rate by measuring the production of DT gamma rays ${ }^{9}$.

4. Neutron Activation Diagnostic (NAD) [28]: primary and tertiary neutron yields. The primary yield is determined by copper activation according to the reaction ${ }^{63} \mathrm{Cu}(n, 2 n){ }^{62} \mathrm{Cu} .{ }^{62} \mathrm{Cu}$ decays into ${ }^{62} \mathrm{Ni}$ by positron emission with a half life of $9.8 \mathrm{~min}$. The threshold energy for this reaction is $10.9 \mathrm{MeV}$. The tertiary yield is determined through the reaction ${ }^{12} \mathrm{C}(n, 2 n){ }^{11} \mathrm{C}$ with a threshold energy of $22 \mathrm{MeV} .{ }^{11} \mathrm{C}$ decays into ${ }^{11} \mathrm{~B}$ by positron emission, with a half life of 20.39 min.

5. Magnetic Recoil Spectrometer (MRS)[29]: neutron spectrum in the 6 to $10 \mathrm{MeV}$ range; fuel $\rho R$ is inferred from this. This spectrometer is based on the production of recoil deuterons in a thin plastic foil and their subsequent passage through a powerful magnet. The energy differentiated deuterons will be recorded in CR-39 detectors. The number of tracks in each energy bin is related to the fuel $\rho R$.

6. High Energy X-Ray Imager (HEXRI) [30]: spatial distribution of hot spot.

This detector is based on the pinhole - scintillator arrangement and will record

\footnotetext{
${ }^{9}$ The DT reaction that normally produces a neutron and an alpha particle, can also produce a 16.7 MeV gamma according to the reaction: $\mathrm{D}+\mathrm{T} \longrightarrow \gamma+{ }^{5} \mathrm{He}$. The branching ratio for this reaction is of the order of $5 \times 10^{-5}$.
} 
time integrated images using $\mathrm{x}$-rays in the 8-30 keV range.

7. Neutron Imaging System (NIS): spatial distribution of primary neutrons (hot spot) and downscattered neutrons (cold fuel).

The suite of ignition diagnostics is being developed by several national laboratories including LLNL, Los Alamos National Laboratory, Sandia National Laboratory, LLE and the French atomic energy agency CEA. Preliminary tests and design studies of these diagnostics are being conducted at the OMEGA[31] laser at LLE.

\subsection{Structure of the Study}

The main objective of this study is to characterize and quantify the image producing capabilities of several line-of-sight designs for the NIS and to compare them against the established design requirements. Chapter two presents the basic principles of neutron imaging, its importance as a target diagnostic, a review of the results obtained at Nova and Omega, and a summary of the design requirements for NIF. Chapter three deals with the experimental scintillator characterization with respect to light output decay and absolute sensitivity. Chapter four presents the Monte Carlo simulations used in the line-of-sight characterization and introduces the concept of the End-to-End model of the NIS. Chapter five contains a review of image formation theory and how it applies to neutron imaging. It also discuses image morphology and shows the results of deconvolution and filtering. Chapter six presents a discussion 
on image quality with respect to the design requirements, resolution and signal-tonoise ratio (SNR). Finally, chapter seven contains the conclusions and suggestions for future work. 


\section{Chapter 2}

\section{Neutron Imaging Principles}

When an implosion fails or 'fizzles' during an ignition attempt at the NIF, the target designers want as much information as possible about the actual configuration and conditions inside the compressed capsule. Understanding a failure is crucial to the success of the next attempt. Neutron imaging provides information about the actual shape of the hot-spot, as well as the cold fuel surrounding it. Significant deviations from a perfectly symmetric implosion contribute to lowering the neutron yield and produce a fizzle. Neutron imaging is, in this sense, a 'failure diagnostic'. This chapter presents the basic concept of hot-spot and downscattered imaging, the pros and cons of neutron imaging, the basic components of an imaging system, the official design requirements for the NIF neutron imaging system (NIS) and a review of the historical development of the technique. 


\subsection{Concept and Neutronics}

Some key aspects related to the fuel distribution are: formation of a central and symmetric hot-spot, mixing of the cold fuel with the ablator during compression, uniformity of the cold fuel shell, and final shape of the compressed or assembled fuel. Departures from a completely spherical compression contribute to lowering the neutron yield and cause a failure.

Thermonuclear neutrons are a desirable choice for an ICF imaging technique. Neutrons are unequivocally related to the fusion reaction and always escape from the compressed capsule; they can produce information about the hot-spot and the cold fuel distributions. For an equimolar DT mixture at $10 \mathrm{keV}$ the bulk of the neutrons are produced isotropically through the $\mathrm{D}(\mathrm{T}, n)^{4} \mathrm{He}$ reaction. The neutron takes away $14.1 \mathrm{MeV}$ and the alpha particle $3.5 \mathrm{MeV}$ due to momentum conservation. The $14 \mathrm{MeV}^{1}$ neutrons are called 'primary neutrons'. A primary neutron can escape uncollided or be involved in a nuclear reaction like elastic scattering $\left(\mathrm{D}\left(n, n^{\prime}\right) \mathrm{D}\right.$ and $\left.\mathrm{T}\left(n, n^{\prime}\right) \mathrm{T}\right)$ or $(n, 2 n)(\mathrm{D}(n, 2 n) p$ and $\mathrm{T}(n, 2 n) \mathrm{D})$. The lower energy neutrons produced in these reactions are called 'downscattered neutrons'. Figure 2.1 shows the simulated neutron spectra of an ignited NIF capsule. The primary neutrons show a finite energy broadening, from 13 to $15 \mathrm{MeV}$, which is an effect of the thermal energy of the deuterium and tritium ions in the hot-spot. Elastic scattering produces a tail from 12 to $6 \mathrm{MeV}$. Bellow this point, the elastic scattered neutrons combine with the ones

\footnotetext{
${ }^{1}$ It is common practice in the ICF community to take the energy of the DT neutrons as 14 instead of $14.1 \mathrm{MeV}$.
} 


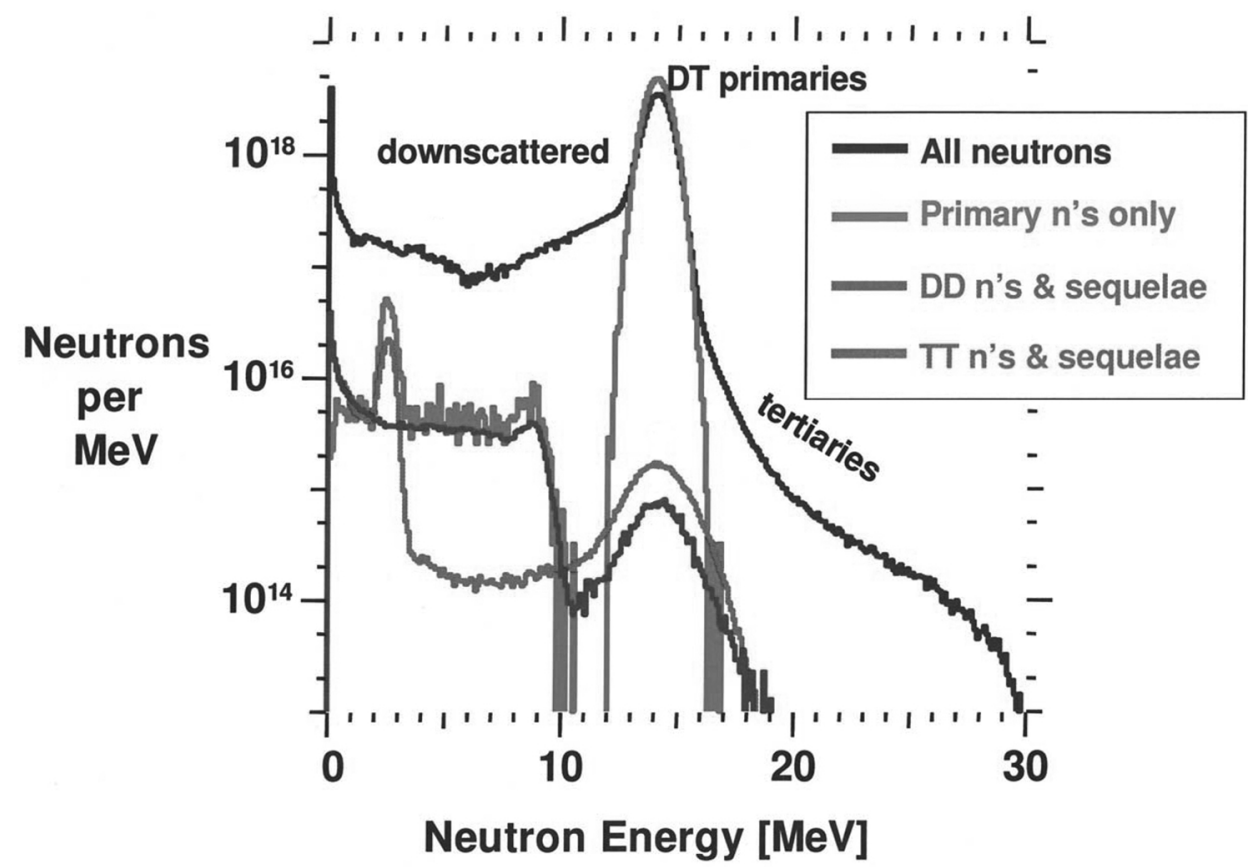

Figure 2.1: Simulated neutron spectra for an ignited NIF capsule. The downscattered region is generated by a combiantion of elastic scattering $(6-10 \mathrm{MeV})$ and $(n, 2 n)$ reactions (below $6 \mathrm{MeV}$ ). Simulation by S. Hatchett

generated in the $(n, 2 n)$ reactions, producing another tail with increasing abundances $(6$ to $\leq 1 \mathrm{MeV})$. Although some DD and TT fusion reactions also produce neutrons, their yields are several orders of magnitude lower than that of DT.

$14 \mathrm{MeV}$ neutrons provide a direct image of the target 'hot spot'. An image of the 6 - $10 \mathrm{MeV}$ downscattered neutrons is related to the cold high density shell, where elastic scattering takes place. The appearance of these two images is directly linked to the neutron kinematics; while the primary neutrons directly represent their origin, since they escape uncollided, the downscattered neutrons are associated with conic regions of the capsule. 


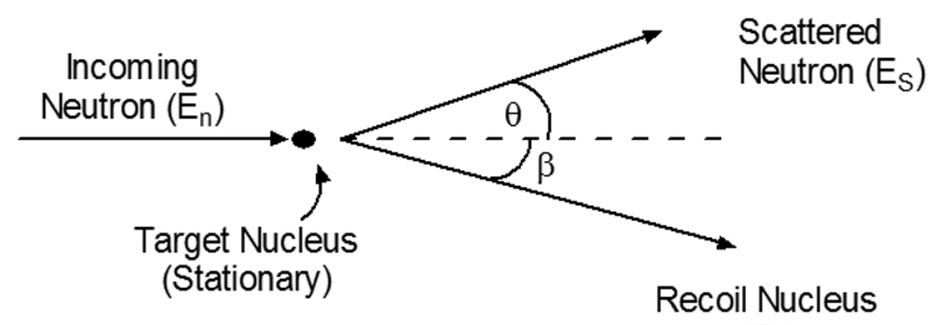

$\left(E_{r}\right)$

Figure 2.2: Neutron elastic scattering diagram in the laboratory reference frame.

Elastic scattering can be described using simple lab-frame kinematics as shown in figure 2.2. Assuming a stationary target of mass $m_{i}$, the energy of the scattered neutron $E_{s}$ is directly related to the scattering angle $\theta$ through the expression[32]:

$$
E_{S}=\frac{1}{(1+A)^{2}}\left[\cos \theta+\sqrt{A^{2}-\sin ^{2} \theta}\right]^{2} E_{n}
$$

where $A=m_{i} / m_{n}$ and $E_{n}$ is the incoming neutron energy. Taking $m_{\text {proton }}=m_{\text {neutron }}$ the factor A takes values of 2 and 3 for deuterium and tritium respectively. The polar plot shown in figure 2.3 can be interpreted as the original directions in which a primary neutron was generated in order to scatter and then be detected with energy $E_{s}$ by an observer placed along the $\theta=0$ axis.

The recorded 6-10 MeV downscattered neutrons come from a conical region that extends from 45 to 75 degrees for deuterium and 60 to 75 degrees for tritium; this distribution makes forward downscattered imaging impossible. The only neutrons available in the forward direction come from the $(n, 2 n)$ reactions, but their low energy puts them on the edge of the downscattered region of interest, making their 


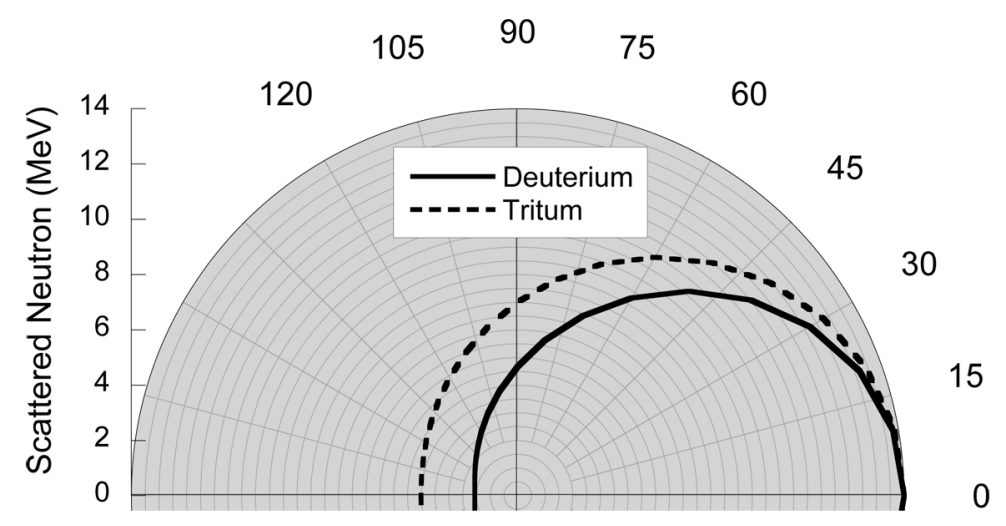

Figure 2.3: Polar plot of $E_{s}$ as a function of scattering angle $\theta$ for a $14 \mathrm{MeV}$ neutron with $\mathrm{D}$ and $\mathrm{T}$.

contribution to the center of the image very small.

An extensive study of possible failure modes[33] was conducted as part of the preparations for the NIC ignition attempts. This study catalogued the effect that different parameters have on ignition and, most importantly, their signatures, i.e., the specific features they impart to the signals of the target diagnostics suite. According to this study neutron imaging is very useful in identifying failures caused by drive asymmetries.

Drive or driver asymmetries at NIF refer to the uneven x-ray heating of the capsule, which translates into different degrees of compression that impart a characteristic shape to the compressed capsule. The asymmetries originate in the discrete illumination pattern of the hohlraum. The 192 beams form four rings of spots on the inner wall of the hohlraum, as shown in figure 1.6; this arrangement leaves dark portions of the wall dark that require extra time to achieve the same temperature as the areas 

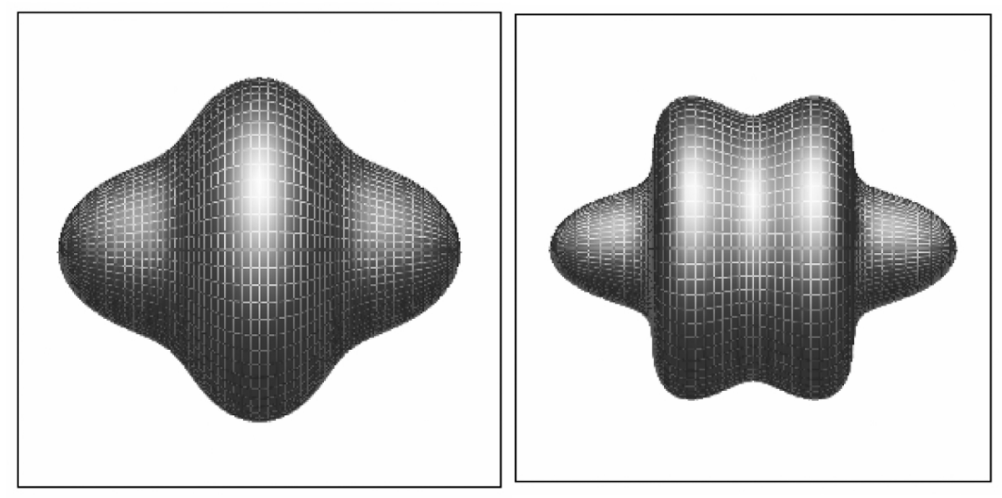

Figure 2.4: $3 \mathrm{D}$ rendering of the $P_{4}$ (left) and $P_{6}$ Legendre polynomials, which are the most common drive asymmetries at NIF. Plots have been rotated 90 degrees to match the simulated images.

directly illuminated by the laser. By pointing the lasers to different spots, the overall homogeneity of the x-ray illumination can be maximized. Depending on the location and separation of the rings several asymmetries can be generated. These asymmetries are usually described using spherical harmonics

$$
Y_{l}^{m}(\theta, \phi)=N \exp (i m \phi) P_{l}^{m}(\cos \theta)
$$

where $N$ is a normalization constant and $P_{l}^{m}$ is the associated Legendre fucntion. Assuming no azimuthal dependency, $Y_{l}^{m}$ reduces to Legendre polynomials $P_{l}$. The two most probable asymmetries at NIF are $P_{4}$ and $P_{6}$, which are writen bellow and illustrated in figure 2.4 .

$$
\begin{aligned}
& P_{4}=\frac{3}{16} \sqrt{\frac{1}{\pi}}\left(35 \cos ^{4} \theta-30 \cos ^{2} \theta+3\right) \\
& P_{6}=\frac{1}{32} \sqrt{\frac{13}{\pi}}\left(231 \cos ^{6} \theta-315 \cos ^{4} \theta+105 \cos ^{2} \theta-5\right)
\end{aligned}
$$

The NIS line-of-sight will be located very close to the target chamber equator. 
The images obtained from this point of view are generated by the combination of the actual fuel spatial distribution and the directions of the primary and downscattered neutrons. As an example, figure 2.5 represents a slice through a simulated density plot of a failed NIF target with due to a $P_{6}$ asymmetry. The image generated using 6-10 MeV neutrons (figure 2.6 left) shows two distinct high brightness regions which are related to the $P_{6}$ high-density rings. There are also two lobes related to the polar features of the $P_{6}$, but since the downscattered neutrons are restricted to a narrow cone, these are imaged faintly. The center portion of the image is illuminated dimly by the $(n, 2 n)$ neutrons. An image generated only by primary neutrons (figure 2.6 right) has a very different appearance; it is smaller than the downscattered, the center region is very bright, and the surrounding contours do not reveal much of the annular structures, just some slight curvature. Figure 2.7 shows the neutron images for a $P_{4}$ asymmetry; the same general principles apply.

These two image sets show a fundamental feature of NIS. Only the neutrons that are either generated towards or scattered into the camera field of view (FoV) are recorded. This has two important implications: neutron images only show information about half of the target, and lines-of-sight with different orientation will show different versions of the same fuel distribution; this is particularly true for downscattered neutrons. Multiple lines-of-sight would be useful in interpreting complex asymmetries, but currently there is only one planned.

The required resolution and signal-to-noise ratio (SNR) are 10 microns and 10 


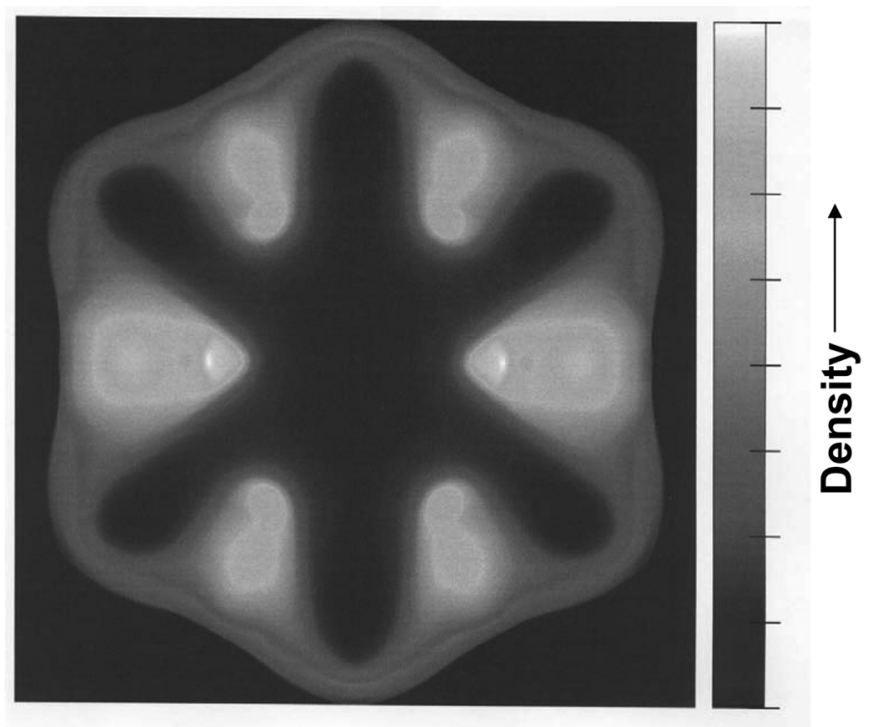

Figure 2.5: Simulated density plot of a failed implosion due to a $P_{6}$ drive asymmetry. The hohlraum axis is shown horizontally for consistency with the other simulated images. The image represents a region of 100 x 100 microns. Simulation by S. Hatchett.

respectively, both measured at the $20 \%$ contour. This lower contour choice is understandable because the asymmetry geometry is easier to observe on the low intensity edges of the images than on their brightest contour. These requirements pose some technical difficulties that will be explored in detail through numerical simulations in chapter four. The actual definitions of resolution and SNR will be presented in chapter five.

Downscattered neutron imaging is possible at the NIF due to the high neutron yields available. Compared to OMEGA, the largest facility currently available, NIF will produce yields two to six orders of magnitude higher, and the distance from target chamber center (TCC) will increase from $8 \mathrm{~m}$ to $25 \mathrm{~m}$, even $40 \mathrm{~m}$. By placing the 

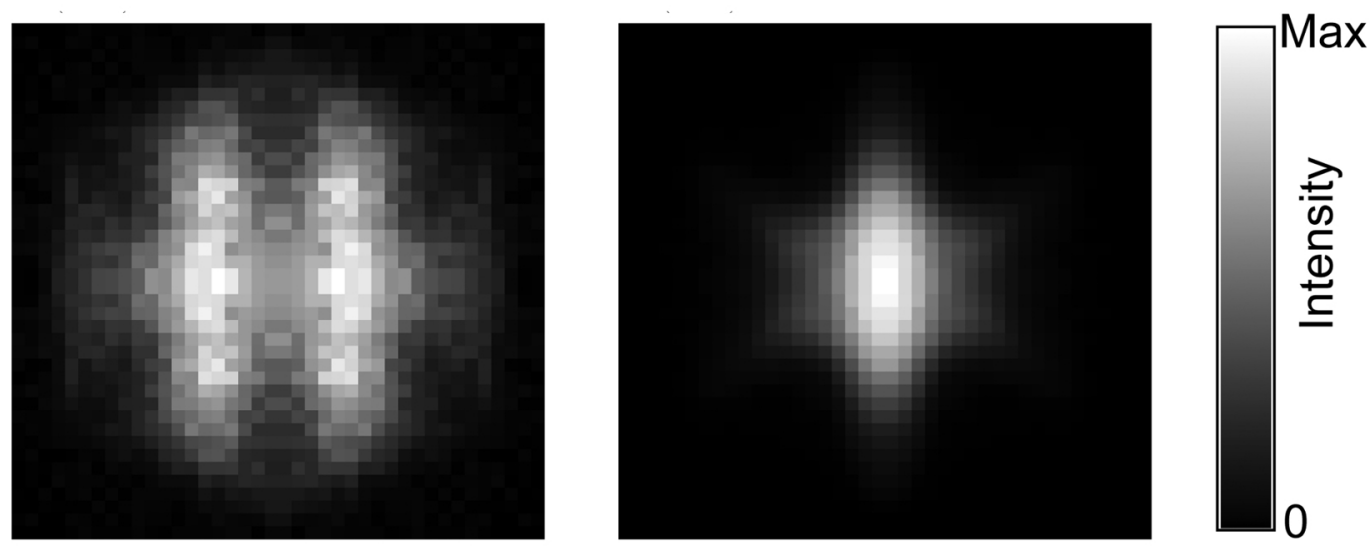

Figure 2.6: Simulated $P_{6}$ neutron images. Left: 6-10 MeV Downscattered Right: 13-15 MeV Primary. Images represents a region of $100 \times 100$ microns around TCC with 2.5 micron square pixels and perfect resolution and no noise. Simulation by S. Hatchett.
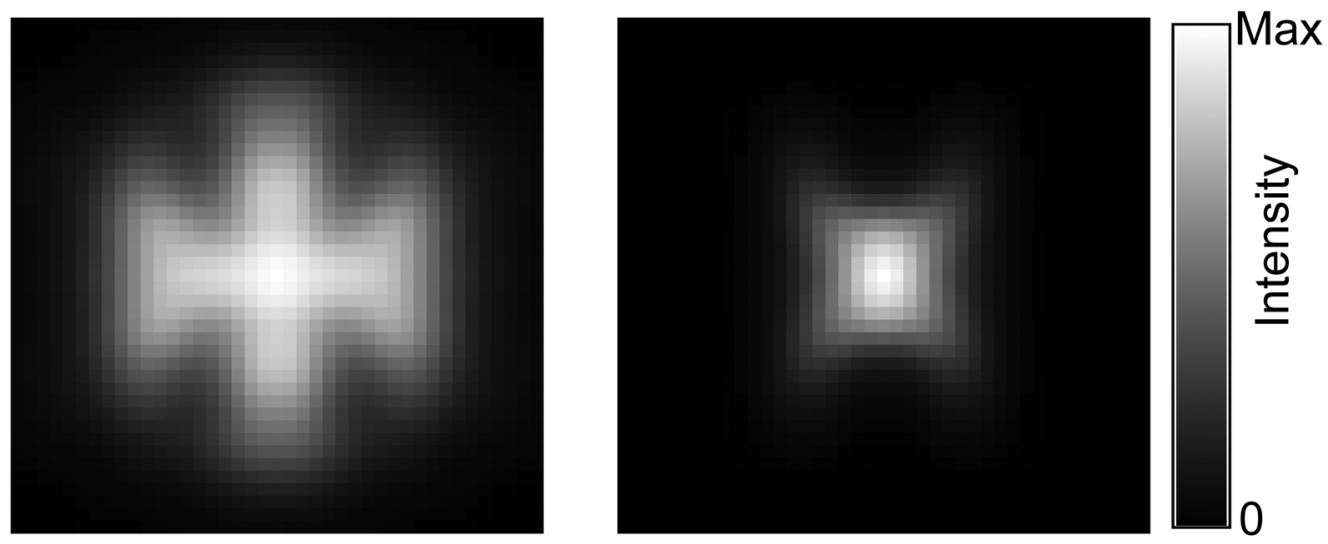

Figure 2.7: Simulated $P_{4}$ neutron images. Left: 6-10 MeV Downscattered Right: 13-15 MeV Primary. Images represents a region of 100 x 100 microns around TCC with 2.5 micron square pixels and perfect resolution and no noise. Simulation by S. Hatchett. 


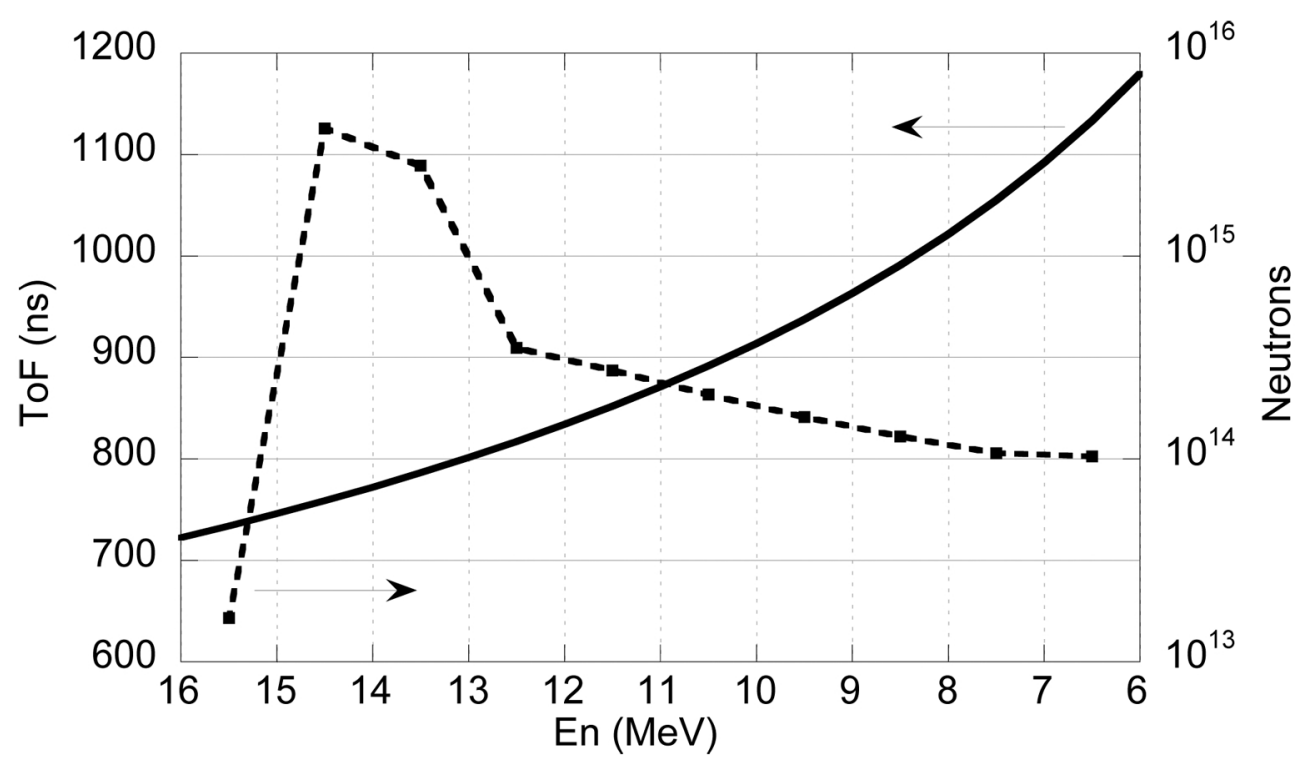

Figure 2.8: Simulated neutron spectrum for a $P_{6}$ fizzle with a primary yield of $6.8 \times$ $10^{15}$ and the corresponding ToF for $40 \mathrm{~m}$.

detector far from the source, the emitted neutron spectrum can be stretched in time due to the finite speed of the particles, this is the basis of the neutron time of flight (nTOF) technique. Based on the kinetic energy $E_{n}$ of a neutron, the time of arrival $t$ at a distance $L$ is given by

$$
t=\frac{L}{\sqrt{\frac{2 E_{n}}{m_{n}}}}
$$

Figure 2.8 shows the simulated neutron spectrum of a NIF fizzle, along with the corresponding time of arrival to a detector placed at $40 \mathrm{~m}$. The primary peak has a width of $55.3 \mathrm{~ns}$ and the $6-10 \mathrm{MeV}$ downscattered $265.9 \mathrm{~ns}$. Their edges are separated by $112.3 \mathrm{~ns}$, this is enough time to record both images. At OMEGA, with a LOS of $8 \mathrm{~m}$ this separation is only $22.5 \mathrm{~ns}$. 


\subsection{Advantages and Disadvantages}

There is no such a thing as a perfect diagnostic, there is always a set of pros and cons, and a balance is needed for success. The NIS is no exception. The main advantages of neutron imaging are:

- Provides information about the hot-spot and cold fuel at NIF conditions

- Low background levels (neutrons, gammas and EMP), since the detector is placed far from TCC, behind the biological shield

- High magnification

- The long LOS spreads the neutron spectrum in time and allows high energy resolution

- The aperture does not need to be very close to TCC, reducing the possible damage during high-yield shots

The main disadvantages of neutron imaging are:

- Images contain information about only half of the source

- Image content highly dependent of LOS orientation

- Downscattered neutrons probe limited conic regions of the target

- Deconvolution/decoding is necessary 


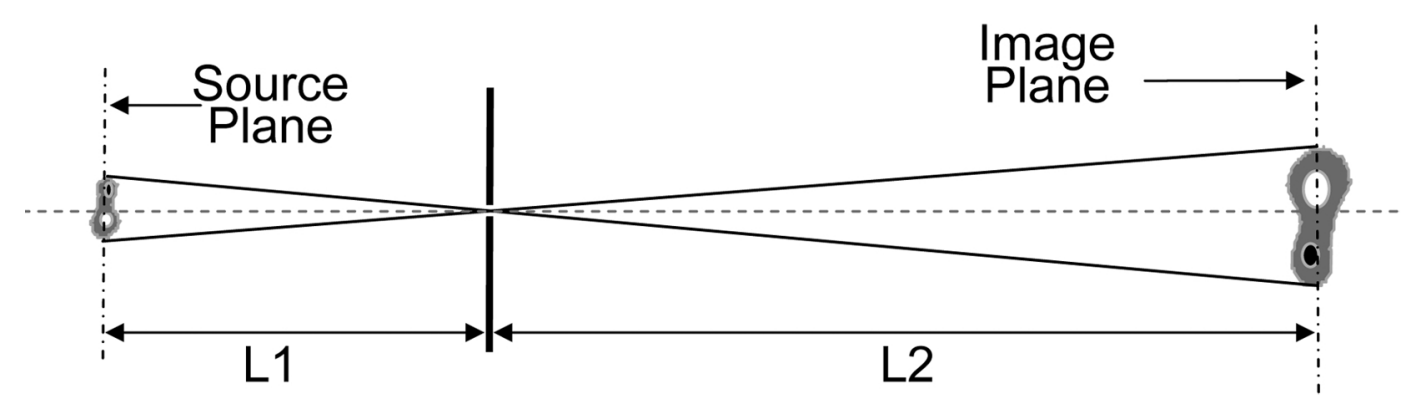

Figure 2.9: Basic pinhole camera.

- Aperture can become activated during high yield shots, restricting its manipulation between shots

- High infrastructure cost: big detector array and LOS ends outside NIF building requiring additional construction

\subsection{A Typical Line-of-Sight}

The NIS is based on the basic ray-optics pinhole camera illustrated in figure 2.9: the light emitted by or reflected from an object passes through a small aperture made in an opaque material placed at a certain distance $L_{1}$ from the object. A screen is placed at a bigger distance $L_{2}$ from the pinhole, generating an inverted and magnified image. The magnification $M$ is given by the ratio of the distances, $M=L_{2} / L_{1}$.

The actual NIS LOS extends from TCC, where the capsule will be located, to the recording station. The LOS consists of: aperture, scintillator array, optical system and digital camera. These components are shown schematically in figure 2.10. 


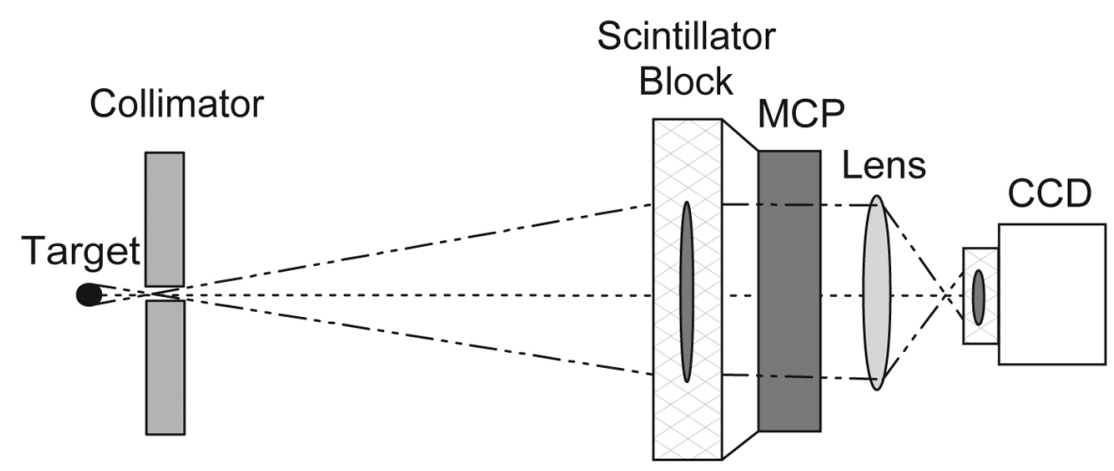

Figure 2.10: Basic components of a neutron imaging system line-of-sight.

\subsubsection{Aperture}

Pinhole imaging with visible light only requires a very small aperture on an opaque material. Pinholes for x-ray imaging are tens of microns thick and made of high-Z materials like gold or tungsten. For high-energy neutrons the pinhole is a very high aspect ratio (length to diameter) aperture made of high-Z materials. The thicknesses range from 10 to $20 \mathrm{~cm}$.

The high-Z material is used to attenuate the radiation (x-rays or neutrons) and create contrast in the image, as the opaque cardboard does with visible light. With x-rays the attenuation comes almost entirely from photo-electric absorption[1], which is proportional to $Z^{4}$. With neutrons the bulk of the total cross section is due to elastic scattering. The high $\mathrm{Z}$ provides high atomic density, which translates into a shorter mean free path (mfp), and thus shorter apertures. The interaction probability or uncollided intensity of a neutron of energy $E_{n}$ traversing a material of thickness $x$ 
is given by

$$
\frac{I}{I_{o}}=\exp \left(-\frac{x}{\lambda\left(E_{n}\right)}\right)
$$

where $\lambda\left(E_{n}\right)$ is the neutron mfp given by

$$
\lambda\left(E_{n}\right)=\frac{1}{\Sigma}=\frac{1}{N \sigma\left(E_{n}\right)}
$$

using pure solid tungsten $\left(\rho=19.35 \mathrm{~g} / \mathrm{cm}^{3}\right)$, the total cross section at $14 \mathrm{MeV}(\sigma=$ $5.321 \mathrm{~b}$ ) and $1 \%$ direct transmission, the required tungsten thickness is

$$
x=-\frac{1}{N \sigma} \ln \left(\frac{I}{I_{o}}\right)=13.7 \mathrm{~cm}
$$

The uncollided neutron flux has an interesting effect on the 'optical' properties of the apertures. The effective size of the aperture is bigger than the opaque material equivalent, and for some geometries the leverage point, i.e., the point that sets $L_{1}$ and $L_{2}$, is displaced from where the optical one would be. This effect can be clearly seen in the Monte Carlo simulations of chapter four.

Besides thickness, the other parameter that determines the aperture profile is the FoV, i.e., the region around TCC that will be imaged. The NIS is required to have a circular FoV with a diameter of 200 microns.

The shape and size of the aperture is not limited by diffraction, as in the case of visible light photons, but by the fabrication method and the source intensity. The apertures can be classified in two main groups: large apertures and pinholes. 


\subsubsection{Large Apertures}

These apertures have dimensions that are large compared to the size of the object being imaged; for neutron imaging this is in the millimeter range. Although in theory the cross section can have an arbitrary shape, in practice only apertures with rotational symmetry have been built. Because they completely distort the shape of the source, they fall into the category of coded apertures (See chapter five). Two large apertures are considered in this study: penumbral and annular.

2.3.1.1.1 Penumbral Aperture Historically most neutron imaging experiments have used a penumbral aperture (See section 2.4). This was due to a combination of ease of construction and high signal throughput; the latter was necessary because of the low neutron yields produced at early facilities. The penumbral aperture produces a very bright spot of constant intensity on the detector (umbra) and a soft-edge region of varying intensity around it (penumbra). All source information is encoded in the penumbra.

Figure 2.11 illustrates the design of a penumbral aperture. Notice how the two truncated cones match the FoV. The aperture is fabricated by machining a sacrificial mandrel with the central dimensions and then pouring the high-Z metal around it. In a final step the mandrel is chemically dissolved, leaving the empty profile.

2.3.1.1.2 Annular Aperture The annular or ring aperture was introduced in medical imaging in the late seventies, but it has been fielded only recently in neutron 


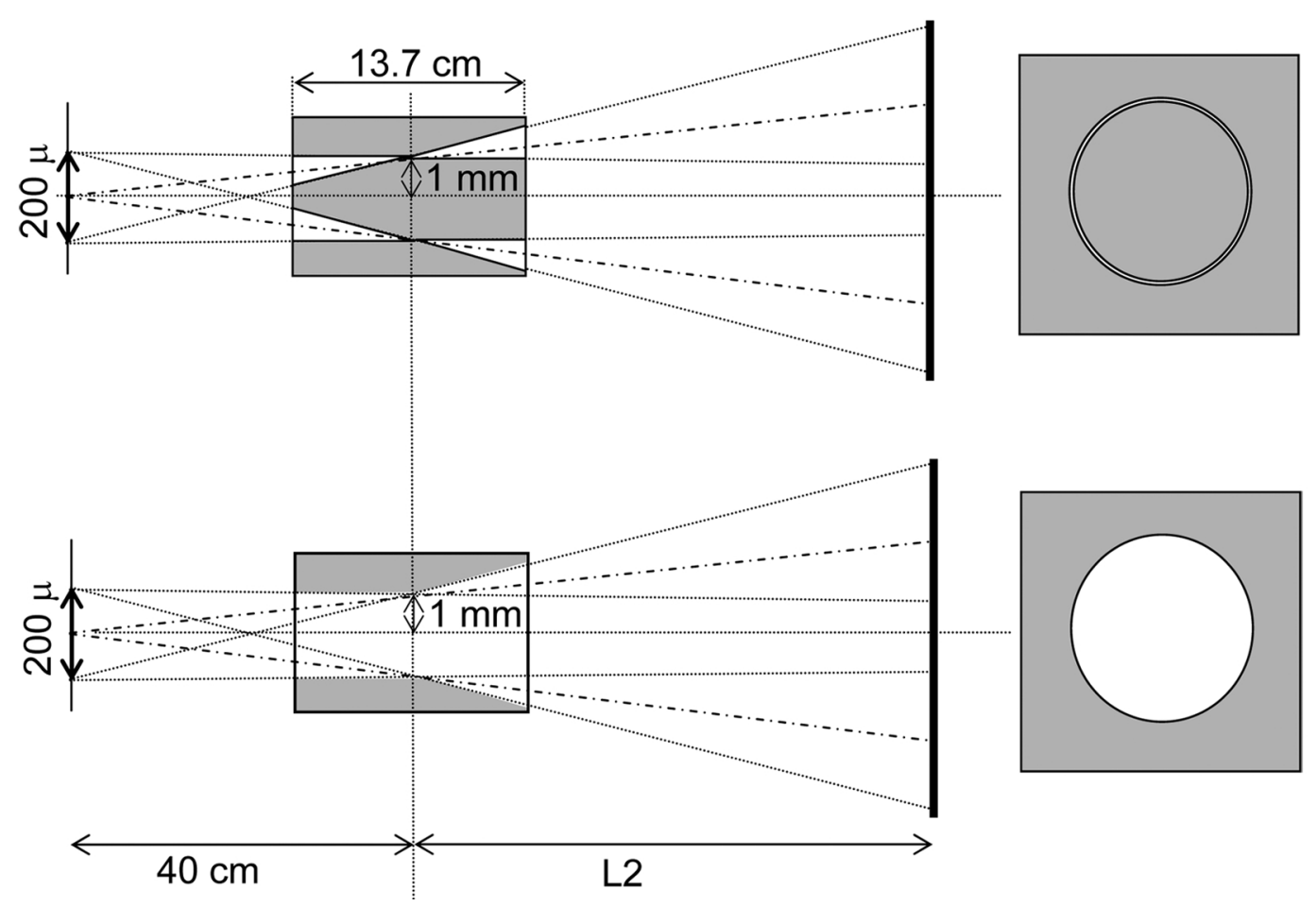

Figure 2.11: Construction details of the big apertures. Annular (top) and penumbral (bottom). $L_{2}$ can be adjusted betwen 25 and $40 \mathrm{~m}$. Drawings not to scale.

imaging experiments. An annular aperture is built by inserting a biconic plug into a penumbral aperture, in such a way that there is only one point of contact (a ring) at the center (see figure 2.11) of the aperture.

An annular aperture can be seen as an infinite array or pinholes or a slit wrapped around in a circle. The truncated cones of the central plug also match the required FoV. Annular apertures are harder to manufacture and require special internal alignment under a microscope. They offer higher image quality than penumbral apertures. 


\subsubsection{Pinholes}

These are apertures with high aspect ratios; their characteristic dimensions are comparable to, or smaller than the size of the object to be imaged, i.e., ten to a hundred microns. Their efficiency is very low and a single pinhole can not be used for the source intensities expected at the NIF. A pinhole array is necessary to achieve the required SNR and resolution; this is particularly true for the downscattered image.

The pinhole arrays are constructed with a regular pitch and all apertures point to TCC. These arrays are hard to fabricate and several techniques have been proposed at LLNL and LANL. Three pinhole arrays are considered in this study: square pinhole, triangular wedge and mini-penumbral.

2.3.1.2.1 Square Pinhole The impossibility of drilling a perfectly straight fivemicron hole through $15 \mathrm{~cm}$ of tungsten prompted engineers at LLNL to come up with an alternate fabrication method. A diamond turning machine can create very precise triangular grooves on tungsten or gold substrates, these grooves can be matched to form a square-cross section pinhole.

Figure 2.12 shows the construction details of this pinhole. The FoV determines the length of the central constant cross section portion and the angles of the entrance and exit tapers. Two central portion dimensions are used in this study: 5 microns for the $40 \mathrm{~m}$ LOS and 50 microns for the $28 \mathrm{~m}$ case. This kind of pinhole can also be constructed without the central portion, a pure biconical design, but the change in 


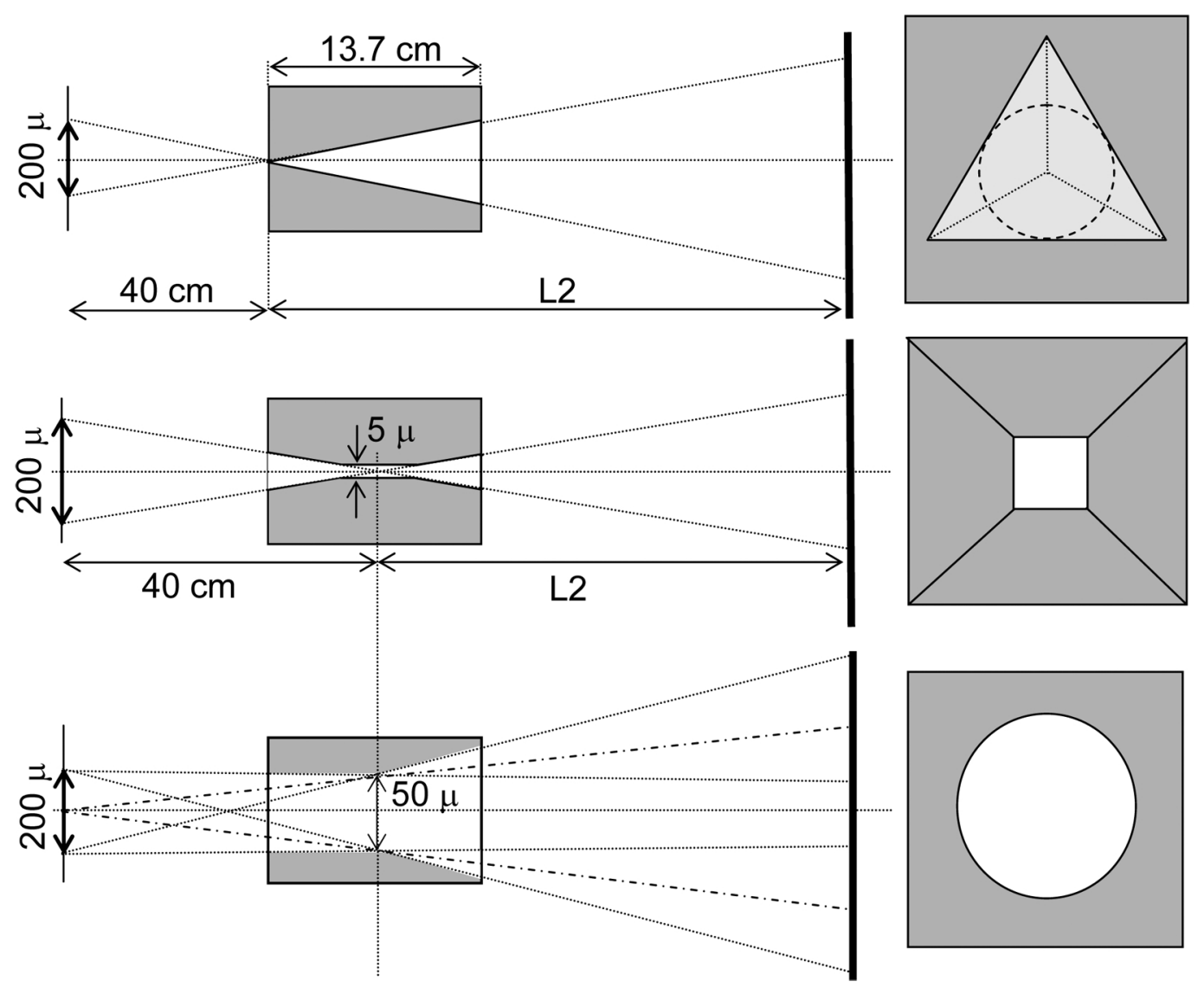

Figure 2.12: Construction details of the pinhole apertures. Triangular wedge (top). Square pinhole (middle), there are two sizes of square pinholes: 5 mcirons (shown) and 50 microns. Mini-penumbral (bottom), two aperture diameters are used: 50 microns (shown) and 100 microns. $L_{2}$ can be adjusted betwen 25 and $40 \mathrm{~m}$. Drawings not to scale.

cross section introduces useful high frequency content in the Fourier transform (see chapter five) that is not present in the biconic alternative.

2.3.1.2.2 Triangular Wedge This aperture is also based on a groove. The empty space has the shape of a tetrahedron, with the apex right on the front face of the high-Z material block, and an angle given by the radius of the inscribed circle that 
matches the FoV. Figure 2.12 shows the details of this aperture. This design is being pursued at LANL.

2.3.1.2.3 Mini-penumbral The mini or small penumbral array is a scaled down version of the traditional penumbral aperture. In this case the central waist has two diameters depending on the LOS: 50 microns for the $40 \mathrm{~m}$ and 100 microns for the $28 \mathrm{~m}$ (See figure 2.12). This type of aperture has not been built yet, but it is considered feasible by engineers at LLNL. It could be fabricated using Electrical Discharge Machining (EDM). It combines the high throughput of the penumbral with the small size and profile of a pinhole. Another particular benefit of the minipenumbral array is the possibility of shinning a laser through it, making the aperture alignment easier. System alignment is a major design issue for the NIS.

\subsubsection{Scintillator Array}

Neutrons can not be recorded directly by a digital camera; an intermediate step is needed to convert the particles into recordable photons. The most effective way to accomplish this is through the use of a scintillator ${ }^{2}[34]$. At the heart of a scintillator is a molecule, either organic or inorganic, that shows very distinct electronic levels that are easily populated when the molecule is excited by a particle or photon. This molecule is present in trace levels in a matrix that can be a plastic or a liquid ${ }^{3}$.

\footnotetext{
${ }^{2}$ From the Latin scintilla, which means spark.

${ }^{3}$ Some polymers, like polystyrene and Lucite, are scintillators by themselves, but their efficiency is very poor. They are commonly used as vehicles for more effective molecules or fluors.
} 
The most common excitation mechanism is ionization, but the actual process varies depending on the kind of molecule and radiation involved.

The most important characteristics of a scintillator used for neutron imaging are: high light output, measured in photons per interaction, and fast light output decay. Although, as it is explained in chapters four and six, the SNR of an image is driven by the number of neutrons hitting each pixel, the amount of light produced by these neutrons in the scintillator helps boost the statistics of the image. In this sense, the higher the light output, the better.

The fast light output decay plays a fundamental role in the recording of a downscattered neutron image. The scintillator detector will be located at a great distance from the aperture (25 to $40 \mathrm{~m}$ ), providing magnification and enough time of flight to separate the downscattered neutrons from the $14 \mathrm{MeV}$ ones. As it was shown in figure 2.8, the neutrons escape from the compressed target with a continuous spectrum; they continuously interact with the scintillator, each time producing a certain amount of light. This light is not emitted instantaneously; on the contrary, the molecular electronic transitions range from a few nanoseconds to tens of miliseconds. As it is shown in chapter three, organic scintillators show a long-lived light output decay component; this delayed light emission can be called an 'afterglow'. For a series of interactions A, $\mathrm{B}$ and $\mathrm{C}$, occurring at times $T_{A}<T_{B}<T_{C}$, the light emitted at $T_{A}$ will be entirely due to $\mathrm{A}$, at $T_{B}$ will be due to $\mathrm{B}$ and the $\mathrm{A}$ afterglow, and at $T_{C}, \mathrm{C}$ and afterglows of $\mathrm{B}$ and $\mathrm{A}$. This is illustrated in figure 2.13. The slower the scintillator decay, the 


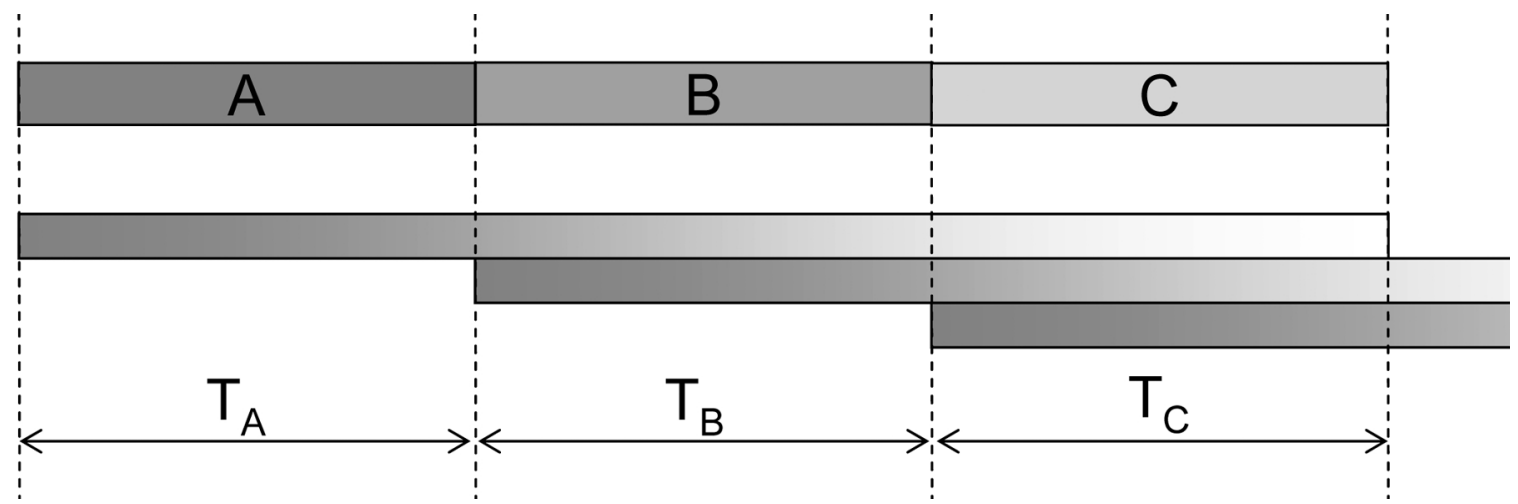

Figure 2.13: Simple explanation of the afterglow contamination due to the scintillator long-lived light emission.

brighter the primary-neutron afterglow present in the downscattered image.

Although placing the detector farther away increases the neutron time-of-flight, and thus reduces the intensity of the afterglow, it also increases the magnification, making the detector bigger; creating engineering and construction problems. At the same time, longer distances imply smaller detector solid angle fractions, if the size of the detector element (pixel) is kept constant. This requires a compromise between scintillator light output decay and detector distance from TCC. For NIF two distances have been considered: 28 and $40 \mathrm{~m}$.

At the beginning of the NIS design study it was decided that only commercially available scintillators were to be considered. After surveying the manufacturers and scientific literature, six candidates were selected: three plastic and three liquid. Chapter three presents the experimental measurement of their light output decay, absolute sensitivity and photon gain. Based on these measurements two scintillator were selected: one plastic and one liquid. 
The thickness of the scintillator is a design variable that provides balance between two competing quantities: detector sensitivity and number of interactions per neutron. Although a high sensitivity detector is highly desirable, the only way to achieve this is by making the detector very thick. This guarantees that a lot of neutrons will interact, but it also allows that a single neutron can undergo multiple collisions. In neutron imaging it is necessary to keep the number of interactions close to one in order to maintain a correspondence between the geometric location of the photons and the neutron source point in the target. Multiple interactions from a neutron will translate into distortions of the source distribution. Depending on the neutron flux at the front face of the scintillator, the interaction probability is usually selected to be between 10 and 20\%. Taking into account that the main light production mechanism below $14 \mathrm{MeV}$ is proton recoil, this study uses a $15 \%$ interaction probability at 14 $\mathrm{MeV}$ with hydrogen only. Using equation 2.4 and the typical composition of a plastic scintillator $\left(\mathrm{C} 4.71 \times 10^{22} \mathrm{~cm}^{-3}, \mathrm{H} 5.19 \times 10^{22} \mathrm{~cm}^{-3}\right.$ ), but considering only elastic scattering with hydrogen $(\sigma=0.68 \mathrm{~b})$, the required thickness is $4.6 \mathrm{~cm}$.

The last design requirement is that the detector needs to be a pixilated array instead of a homogeneous slab. This is necessary to improve the light collection efficiency of the system. The light in a scintillator is produced isotropically at any point of the detector. An optical system with a big depth of field is required to collect all the photons produced at different depths. A big depth of field makes the systems very slow, requiring higher particle fluxes to achieve the same collected image 
intensity. In contrast, a very fast system will only collect the photons produced in a narrow band of the scintillator. By dividing the scintillator into small thick pixels, fibers, the light produced at any point inside the scintillator can be transported to the array faces, where it can be collected by any of the methods described in the next section. Several manufacturers offer scintillators in plastic fibers that act as light guides, transporting most of the photons to the faces of the array. Fused silica capillaries filled with a liquid scintillator can also be used in this way.

\subsubsection{Optical System}

The optical system serves three purposes: image transport, signal intensification and gating. The size of the scintillator array is expected to be of the order of 15 to 20 $\mathrm{cm}$. Since most CCD chips are small, 2 to $3 \mathrm{~cm}$, the image produced on the surface of the scintillator array needs to be reduced and transported to the camera. This can be accomplished by using fiber optic tapers or fast lenses directly coupled to the camera. The signal is intensified by a micro-channel plate (MCP) based intensifier, where the photons coming from the scintillator hit a photo-cathode, producing electrons that travel through the very small channels of the MCP, where they generate more electrons. These electrons hit a phosphor screen producing a more luminous image. Image intensifiers are available in multiple diameters or bundled with a camera.

The image intensifier also serves as a gate or shutter for the imaging system. The MCP can be gated very fast allowing exposures of the order of tens of nanoseconds. 
For neutron imaging the main requirements of the optical system are fast optics, so more photons per resolution element are recorded, and intensity homogeneity across the image, preventing big variations due to optical aberrations and the extinction ratio of the MCP, i.e., how fast and uniformly the amplified image disappears once the high voltage is removed from the MCP.

\subsubsection{Digital Camera}

The image is finally recorded with a CCD camera. Most commercially available cameras are capable of recording just one image during the hundreds of nanoseconds between the primary peak and the end of the downscattered gating window. In order to record both images in this short time interval either two independent cameras or an internally multiplexed unit is required.

\subsection{Historical Development}

The use of neutron imaging as a diagnostic dates back to the underground test program at the Nevada Test Site[35]. Before 1976 a so-called 'mechanical' PINEX (for pinhole experiment) was used to obtain rough images of the burning cores. In this approach, metallic plates ( $\mathrm{Zr}, \mathrm{Al}$ and $\mathrm{Nb}$ ) were used as detectors. The neutrons would go through the pinhole array and interact with the plates, activating them. The plates would be retrieved from the shaft and placed under radiographic film. The radiation 
emitted from the metallic plates would expose the film, creating a continuous density map representative of the neutron source. After 1976 an electronic version of PINEX was introduced. In this approach there were no retrievable parts and the imaging was done through multiple video cameras focused on a thick slab of scintillator. The video information was transmitted to the surface though fast electronics before the shock wave of the explosion would destroy the system. On the surface the image was analyzed and converted to a scaled density map of the core. Some version of this system remained in use until the end of the test program in 1992.

On the civilian side the first steps toward neutron imaging date back to the early 1980s when Nova was being designed. In 1981 Lerche and Sommargren[36] studied the effect of collimator shape on FoV and PSF isoplanatism, and from 1984 through 1986 Nugent and Luther-Davies[37][38][39] used their experience with x-ray imaging to develop a comprehensive penumbral neutron imaging technique. The first ICF neutron image was recorder by Ress et.al. [40][41] at Nova using a penumbral aperture and a plastic scintillator array. The system had a resolution of 80 microns and a SNR better than 10. In France in 1993 a group led by Garçonnet[42] recorded images with 130 microns resolution at the Phébus laser using a penumbral aperture and a plastic scintillator array. This system was improved and in 1994 Delage et.al.[43] recorded images with 56 micron resolution. In 2000 this same system was installed at Omega recording images with 43-micron resolution[44]. During the same campaign a so-called 'bubble detector' was used delivering 150 micron resolution. Work using 
the bubble detectors continued at Omega yielding 25 micron resolution in 2002[45]. In 2002 Disdier et.al.[46] demonstrated a new detector array using fused silica capillaries filled with a liquid scintillator. Using a penumbral aperture they recorded images with 20 micron resolution. In the same year a group from Los Alamos National Laboratory[47] obtained the first images using a square pinhole and a plastic scintillator array. Work with square pinholes continued in 2003 with the test of a two-pinhole array prototype[48], the recorded image clearly showed the two different sized pinholes. Most recently, in 2005, Disdier et.al.[49] succeeded in recording a 20 micron resolution image using an annular aperture and the liquid filled capillary array at Omega. 


\section{Chapter 3}

\section{Scintillator Characterization}

As part of the design studies for the NIS, it is of particular importance to understand and quantify the response of the scintillator to the primary and downscattered neutrons, and based on these figures select the best material for the detector. This chapter presents the experimental setup used in the measurement of the light output decay profile and the absolute sensitivity of six commercially available samples, a discusion of the results and concludes with the selection of two candidates for the detector. This portion of the NIS study is an extension of the work presented in the Master's of Science thesis 'Characterization of Scintillator Materials for Downscattered Neutron Imaging'[50]. The reader is referred to this reference for a more detailed treatment of the chemistry and physics of organic scintillators, the basic principles of neutron detection using scintillators, and the fundamentals of the light output decay measurement technique. 


\subsection{Scintillator Samples}

After surveying the technical literature and the scintillator manufacturers, six commercially available organic scintillators were selected as candidates for the NIS detector. The scintillators were chosen based on their published decay constant and their availability in plastic or liquid form. The scintillators considered in this study are:

- EJ232 ${ }^{1}-\mathrm{BC} 422^{2}$ : Fast plastic scintillator

- EJ232Q 1\% - BC422Q 1\%: Quenched version of EJ232, containing 1\% by weight benzophenone

- EJ232Q 2\% - BC422Q 2\%: Quenched version of EJ232, containing 2\% by weight benzophenone

- BC509: Fast liquid scintillator. Virtually hydrogen free

- EJ399A: Liquid scintillator used by Disdier et.al.

- EJ399B: Slight variation of the 399A formulation, also used by Disdier et.al.

There are two samples of the first three scintillators, each from a different manufacturer. They are supposed to be equivalent formulations. They were selected in order to verify any differences. This plastic scintillator is widely used in radiation

\footnotetext{
${ }^{1}$ Eljen Technology 2010 East Broadway Street Sweetwater, TX 79556 USA (888) 800-8771 www.eljentechnology.com

${ }^{2}$ Bicron (owned by Saint-Gobain) 1655 Townhurst Drive Houston, TX 77043 USA (281) 355-1033 www.detectors.saint-gobain.com
} 


\begin{tabular}{lccc}
\hline \hline Scintillator & $\begin{array}{c}\text { Light Output } \\
\text { \% Anthracene }\end{array}$ & $\begin{array}{c}\lambda \text { of Max. } \\
\text { Emission (nm) }\end{array}$ & $\begin{array}{c}\text { Decay } \\
\text { Constant (ns) }\end{array}$ \\
\hline EJ232 & 55 & 370 & 1.4 \\
EJ232Q 1\% & 11 & 370 & 0.7 \\
EJ232Q 2\% & 5 & 370 & 0.7 \\
BC509 & 20 & 425 & 3.1 \\
EJ399A & NA & 435 & NA \\
EJ399B & NA & 420 & NA \\
\hline \hline
\end{tabular}

Table 3.1: Basic physical properties of the six selected scintillators. NA: Not available from manufacturer. The properties of the BC422 samples are assumend identical to those of the EJ232.

detection and is available in slabs and cylinders, or in small step-index fibers that act as light guides. The quenched versions were included in order to study the effect of benzophenone on the long-lived decay component and the light output. BC509 is a liquid scintillator that was included due to its very low hydrogen content ${ }^{3}$, this is thought to translate into higher detector resolution since the lightest nuclei available for elastic scattering is carbon, which has a maximum recoil energy of $0.221 E_{n}$ and thus shorter range. The two EJ399 samples were included in the study because Disdier et.al. have used them, with great success, in a detector made of fused silica capillaries filled with the liquid scintillators[51], but the actual parameters were not known. Table 3.1 summarizes the physical properties of the scintillators as provided by the manufacturers.

\footnotetext{
${ }^{3}$ Almost all the hydrogen in the organic molecules has been replaced by fluorine. The atomic percentage of hydrogen atoms, as quoted by the manufacturer, is $0.18 \%$.
} 
All the samples had the same shape: right circular cylinders 1.5 in diameter by 1.5 in tall. The plastic samples were painted white, except for one of the circular faces and a small circle on the side wall. The liquid samples were encapsulated in a fused silica reservoir painted white, except for one of the circular faces and a small side window.

\subsection{Light Output Decay Measurement}

The emission of light as a function of time in an organic scintillator is a statistical process that can be described using an expression of the form[52]:

$$
I=\sum_{i=0}^{m} A_{i} \exp \left(-\frac{t}{\tau_{i}}\right)
$$

where $I$ is the light emission intensity, $\tau_{i}$ are the so-called decay constants or lifetimes, $A_{i}$ are scaling constants and $t$ is time, measured in nanoseconds (ns). Depending on the molecules involved, equation 3.1 might require up to ten terms, each associated with a particular electronic process. For the scintillators and timescales considered in this study, only four terms are used. It is common practice among scintillator manufacturers to report only the first decay constant, which is only valid for these scintillators during the first 20 ns after peak emission. Downscattered neutron imaging requires precise knowledge of the decay profile hundreds of ns after the peak. Experimental measurement of the additional decay constants is necessary.

The technique used to obtain the light decay profile, Time Correlated Photon 
Counting (TCPC), is described in great detail by O'Connor and Phillips [53] and in the M.Sc. thesis. The experimental setup used for the measurements is showed in figure 3.1. The scintillator sample $\mathrm{S}$ is placed in a light-tight housing that allows direct coupling of photo multiplier tube 1 (PMT1) with the clear circular scintillator face. The side window couples through an SMA connector to a fiber optic bundle or a light-pipe and then to PMT2. The signal from the PMT1 anode is directly fed to a constant fraction discriminator (CFD1), while the signal from PTM2 is amplified by four fast linear stages in series (4/AMP) and then fed to CFD2. The time-to-amplitude converter (TAC) receives a 'start' pulse from CFD1 and a 'stop' pulse from CFD2; in order to position the signal in the coincidence window a cable delay line is placed between CFD1 and the TAC. The output of the TAC is fed to a PC-based multi-channel analyzer (MCA) set to 512 channels.

The optical link between the scintillator and PMT2 has two main purposes: to place PMT2 far away from the radiation source to avoid direct interaction and thus reduce the background, and to restrict the number of photons that hit PMT2, which has to be in the single photon counting regime. The original set of experiments used a fiber optic bundle, but the measurements were contaminated by a reflection from the air-glass interface at the end of the bundle. The fiber bundle was replaced with a light-pipe, which is a one inch stainless steel pipe with highly polished interior, a pair of simple lenses and SMA connectors on each end to attach the scintillator housing and PMT2. The light-pipe eliminated the reflection completely, generating a smooth 


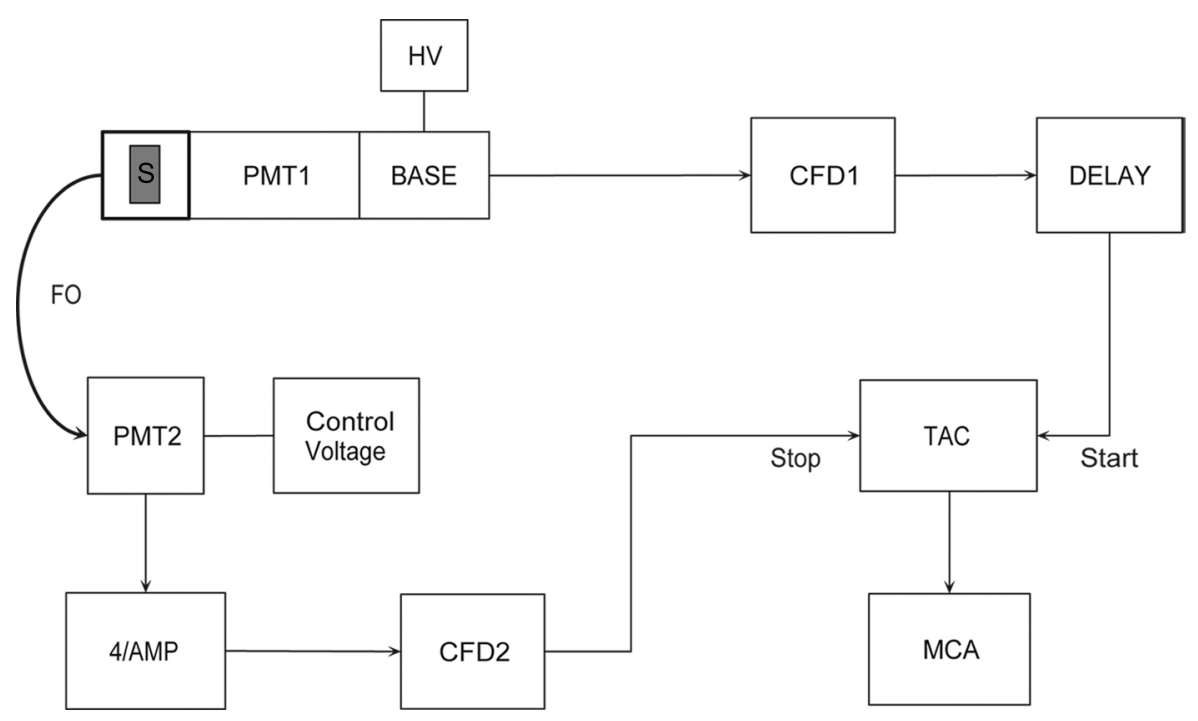

Figure 3.1: Diagram of the experimental setup used for the time correlated photon counting measurements.

decay profile.

The study used three kinds of ionizing radiation: DT (14 MeV) and DD (2.5 $\mathrm{MeV}$ ) neutrons, and $662 \mathrm{KeV} \gamma$-rays from a ${ }^{137} \mathrm{Cs}$ sealed source. The neutrons were produced with the DC electrostatic neutron generator in B381. The DT neutrons were produced by launching $D^{+}$ions into a copper target with a layer of tritium-doped titanium. The DD neutrons were produced using a blank copper target. Although there were no intermediate neutron energies available, especially around 6 - $10 \mathrm{MeV}$, these two data sets are useful for bracketing the scintillator behavior.

Figure 3.2 shows the raw decay data for EJ232 excited by $14 \mathrm{MeV}$ neutrons. The data was obtained using the $1000 \mathrm{~ns}$ coincidence window of the TAC and enough cable delay to place the peak of the decay curve 50 channels from the left edge of the MCA scale. There is a flat background level in front of the signal peak that is 


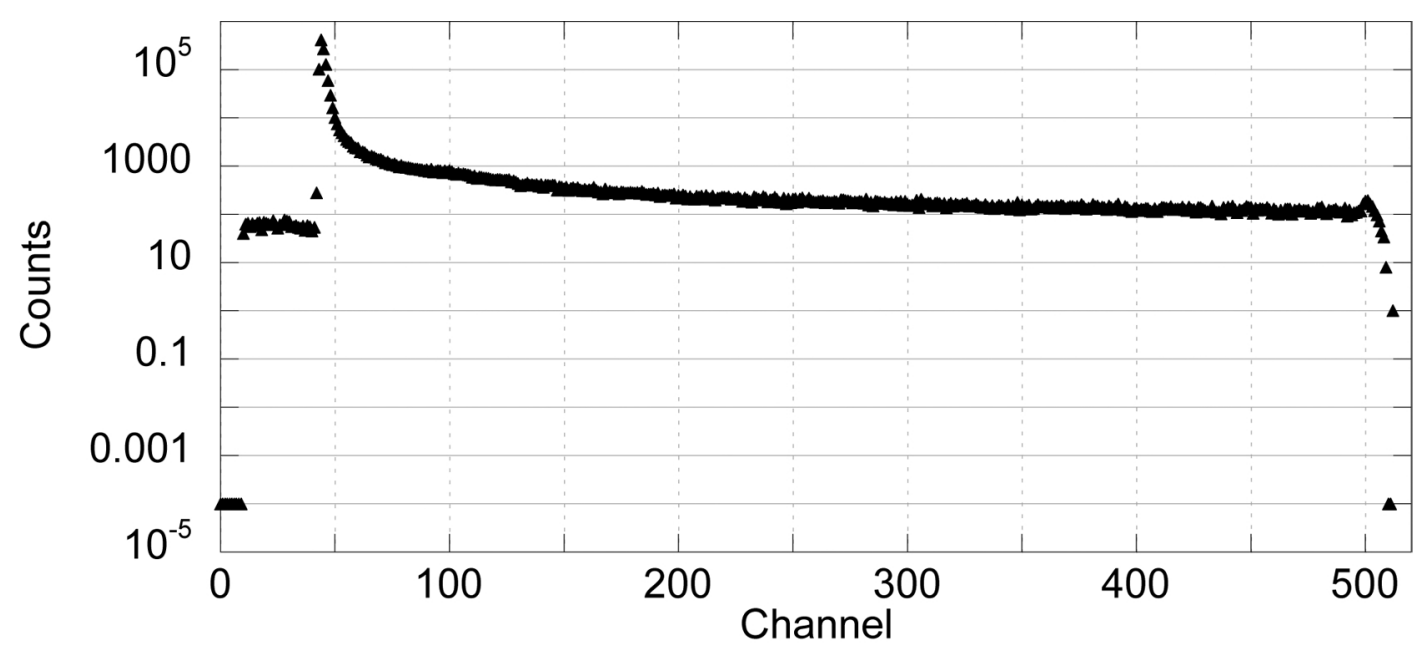

Figure 3.2: Raw data of the light emission decay of EJ232 excited by $14 \mathrm{MeV}$ neutrons. Full scale represents 1000 ns. The counts to the left of the peak are uncorrelated background, the small bump at the end of the tail is due to pile up in the MCA.

due to uncorrelated counts, it is a feature of coincidence techniques and its value is given by the count rates of both PMTs[34]. The live time is that required to get a flat uncorrelated background and at least a $\sigma=10 \%$, assuming Poisson counting statistics, at around 600 ns from the peak. The MCA channels are transformed into time by conducting a TAC scale calibration with a pulse generator. Each channel is equivalent to $2.08 \mathrm{~ns}$. The time axis extends from -100 ns to $800 \mathrm{~ns}$. The uncorrelated background is averaged and subtracted from every channel, and the peak intensity is set equal to one.

The decay curve can be fitted to a four term exponential series using a leastsquares algorithm or to four discrete exponential terms that extend for some number of data points. The second approach is easier to implement and less sensitive to data noise and initial values. The adjusted and fitted data for EJ232 with DT neutrons 


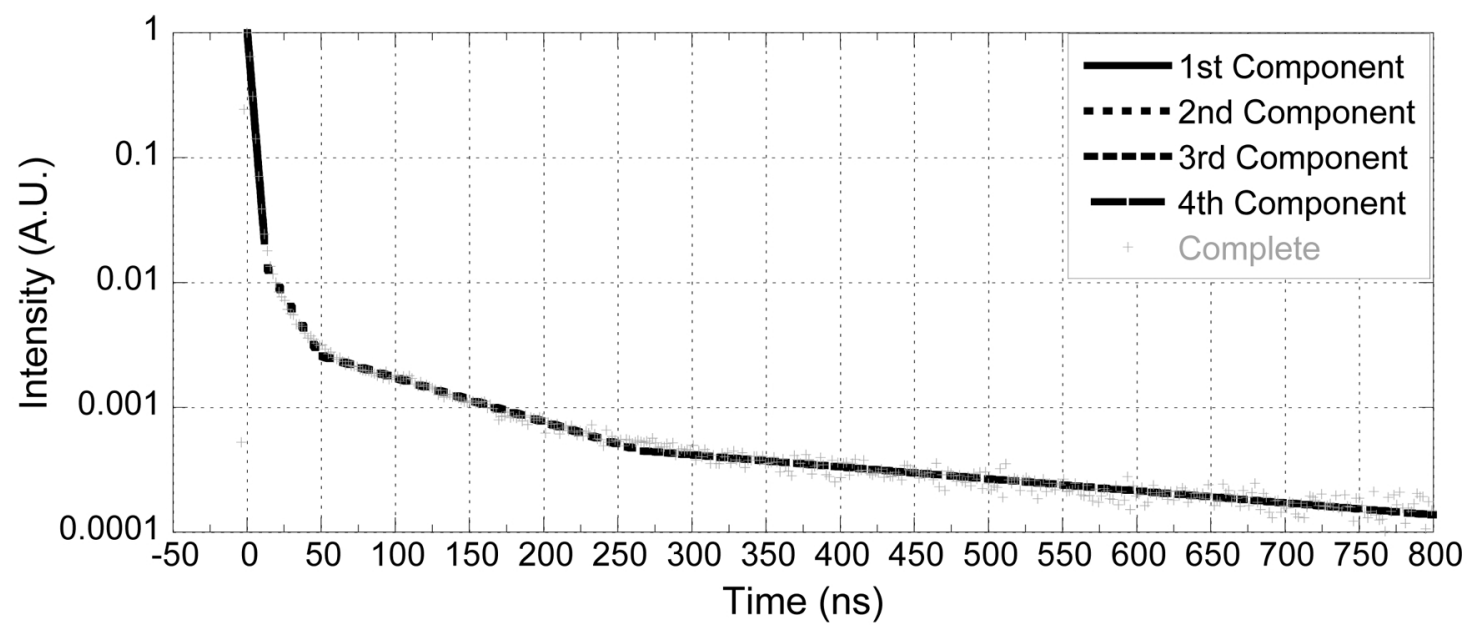

Figure 3.3: Light emission decay of EJ232 excited by $14 \mathrm{MeV}$ neutrons after processing and showing the four decay exponential components $\tau_{1}=2.99 \mathrm{~ns}, \tau_{2}=23.64 \mathrm{~ns}$, $\tau_{3}=123.17 \mathrm{~ns}, \tau_{4}=451.75 \mathrm{~ns}$.

is shown in figure 3.3. The first decay component $\tau_{1}$ has a value of $2.99 \mathrm{~ns}$, which is more than twice the value claimed by the manufacturer. The discrepancy presumably is due to the low time resolution of the 1000 ns measurement (2.08 ns per channel). The 100 ns coincidence window measurements produce more accurate values of $\tau_{1}$ since the time per channel is only is $0.195 \mathrm{~ns}$.

Figure 3.4 shows the decay curves for the six scintillators with $14 \mathrm{MeV}$ neutrons. All samples show a long-lived decay component that extends beyond $500 \mathrm{~ns}$. The EJ232 samples decay faster than the liquid scintillators, but the effect of the quenching is not very noticeable in the long-lived tail, it mostly affects the first portion. The two EJ399 samples show the highest light levels at $350 \mathrm{~ns}$ and their intermediate decay is also slower than the EJ232. BC509 shows a shorter lived decay profile than the rest; it is slower in the beginning and intermediate regions but it has reached background 


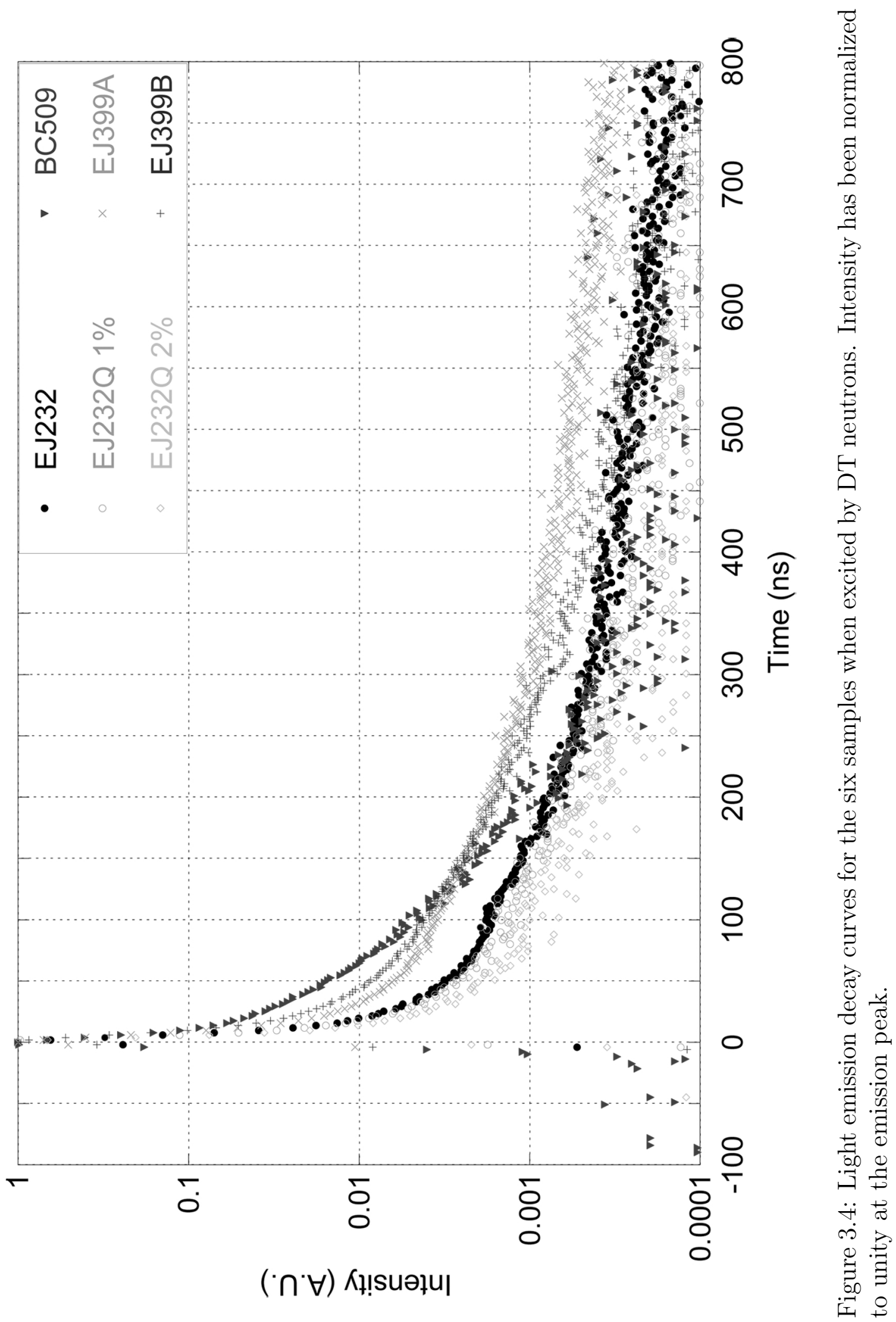




\begin{tabular}{lcccc}
\hline \hline Scintillator & $\tau_{1}(\mathbf{n s})$ & $\tau_{2}(\mathbf{n s})$ & $\tau_{3}(\mathbf{n s})$ & $\tau_{4}(\mathbf{n s})$ \\
& & & & \\
\hline EJ232 & 2.99 & 23.64 & 123.17 & 451.75 \\
EJ232Q 1\% & 2.23 & 16.32 & 132.24 & 392.34 \\
EJ232Q 2\% & 1.99 & 15.90 & 119.06 & 549.06 \\
BC509 & 4.34 & 29.63 & 80.30 & $\mathrm{NO}$ \\
EJ399A & 3.95 & 29.74 & 155.95 & 463.84 \\
EJ399B & 3.77 & 24.73 & 115.03 & 279.17 \\
\hline \hline
\end{tabular}

Table 3.2: Light emission decay constants for the six selected scintillators when excited by $14 \mathrm{MeV}$ neutrons. Measurement resolution \pm 2.08 ns. NO: not observed

levels at around 375 ns. Most of the decay, at least two orders of magnitude, occurs in the first 50 ns. By 150 ns the EJ232 samples are down three orders of magnitude and the EJ399 reach this point at 275 ns. Although all of these samples are fast by scintillator standards, none of them shows a sharp and continuously fast decay profile that would minimize the afterglow contamination of the downscattered image by the primary neutrons. Table 3.2 summarizes the manually fitted decay constants or lifetimes.

Two additional sets of measurements were conduced using $2.5 \mathrm{MeV}$ neutrons and the ${ }^{137} \mathrm{Cs}$ sealed source. The results are very similar to the ones at $14 \mathrm{MeV}$, the only difference being the relative decay speed. Figure 3.5 shows the decay curves of EJ399B for both neutron energies and the $\gamma$-rays. The $\gamma$-ray excitation produces the fastest decay, followed by the 14 and the $2.5 \mathrm{MeV}$ neutrons. The difference in the decay constants is explained by taking into account the excitation mechanisms involved. $\gamma$-rays are the fastest because it is a one step process; the ionization is produced via 


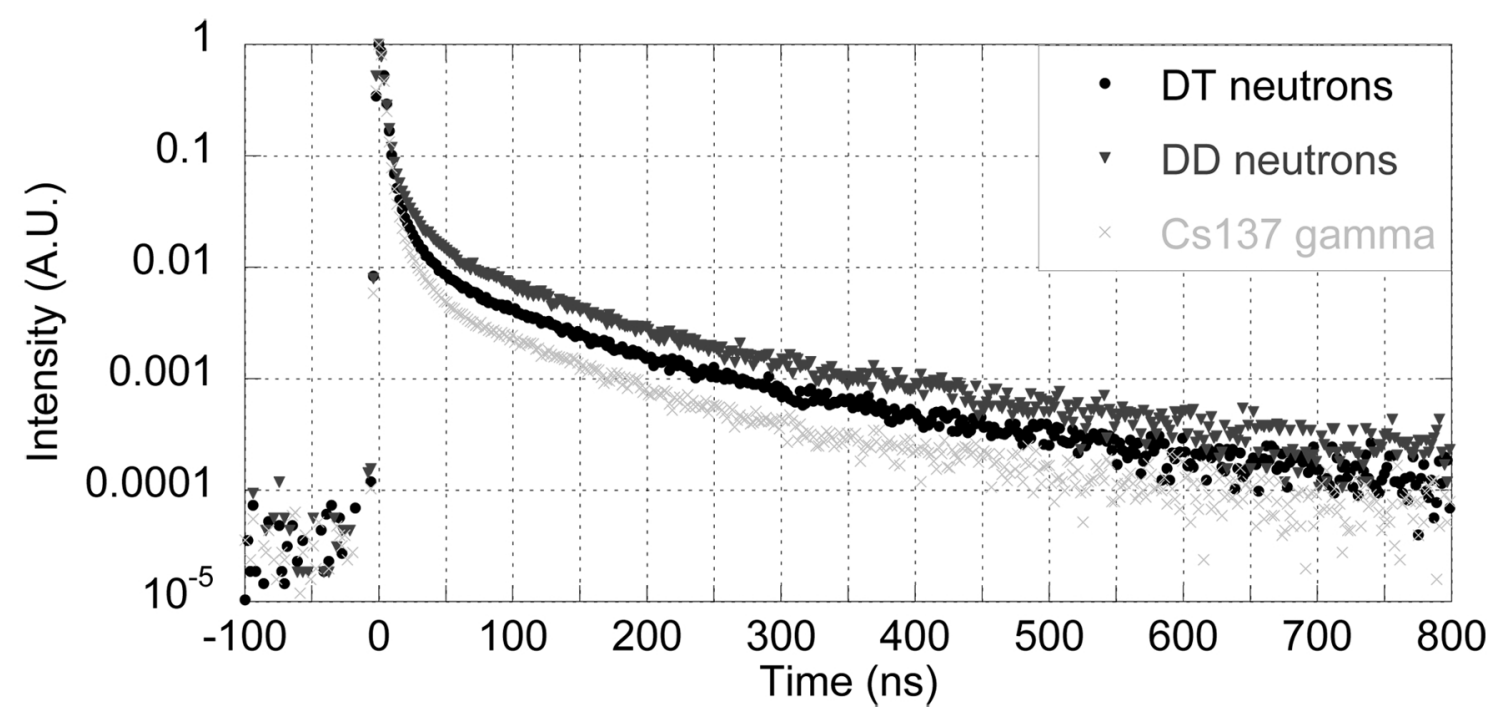

Figure 3.5: Light emission decay for EJ399B for three different kinds of radiation. Notice how $\gamma$-ray excitation produces the fastest decay, followed by DT neutrons and finally DD neutrons.

the photo-electric effect and the electronic transitions can occur immediately.

Neutron excitation is a two step process; first, the neutron must interact with the nuclei, hydrogen in particular, knocking a proton off, then the recoil proton produces ionization. The difference between the two neutron energies is explained taking into account the process of self-quenching, which is due to the high density ionization tracks produced by the high energy protons. In these highly ionized regions the molecular or $\pi$ electrons recombine faster, leading to a quicker decay. Although there are differences in the decay profiles at different neutron energies, they are small, and the long lived tails fall very close to each other. For the NIS $6 \mathrm{MeV}$ is the minimum neutron energy of interest, its decay curve would lie between the two measure profiles. For simplicity, all the image calculations of chapter four will use the DT decay curves. 
A 100 ns coincidence window provides additional information about the very fast decay components. The results for $14 \mathrm{MeV}$ neutrons are shown in figure 3.6. At this scale all the decays are differentiated and the effect of the benzophenone quenching in EJ232Q clearly visible. EJ232Q 2\% shows the fastest decay of the three, but as seen in figure 3.4 the long-lived component is practically unchanged. Changing the quenching agent to pipiridine might be more effective, as shown by Lyons et.al.[54] and Fluornoy[55], but at the time of the study there were no of-the-shelf pipiridine quenched scintillators, and the long term stability of custom scintillators was not guaranteed by the manufacturers. It is important to mention that chemical quenching has a drawback, it has a big impact on the light production, since it replaces a lot of the light emitting electronic transitions with radiationless recombination processes. This will be evident in the absolute sensitivity measurements.

At this higher time resolution the first decay component $\tau_{1}$ for EJ232 can be fitted to $2.32 \mathrm{~ns}$, this is still $61.25 \%$ higher than the manufacturer's value. Additionally the rise time of all scintillators appears slower than reported. The only reason for these discrepancies seems to be that the whole-system response is a significant portion of the fitted parameters and it needs to be deconvolved, so the real value can be quoted. The response of the timing system needs to be measured using an intense gamma source like ${ }^{60} \mathrm{Co}$. This discrepancy does not pose a problem for the NIS modeling since the images are integrated over longer timescales. 


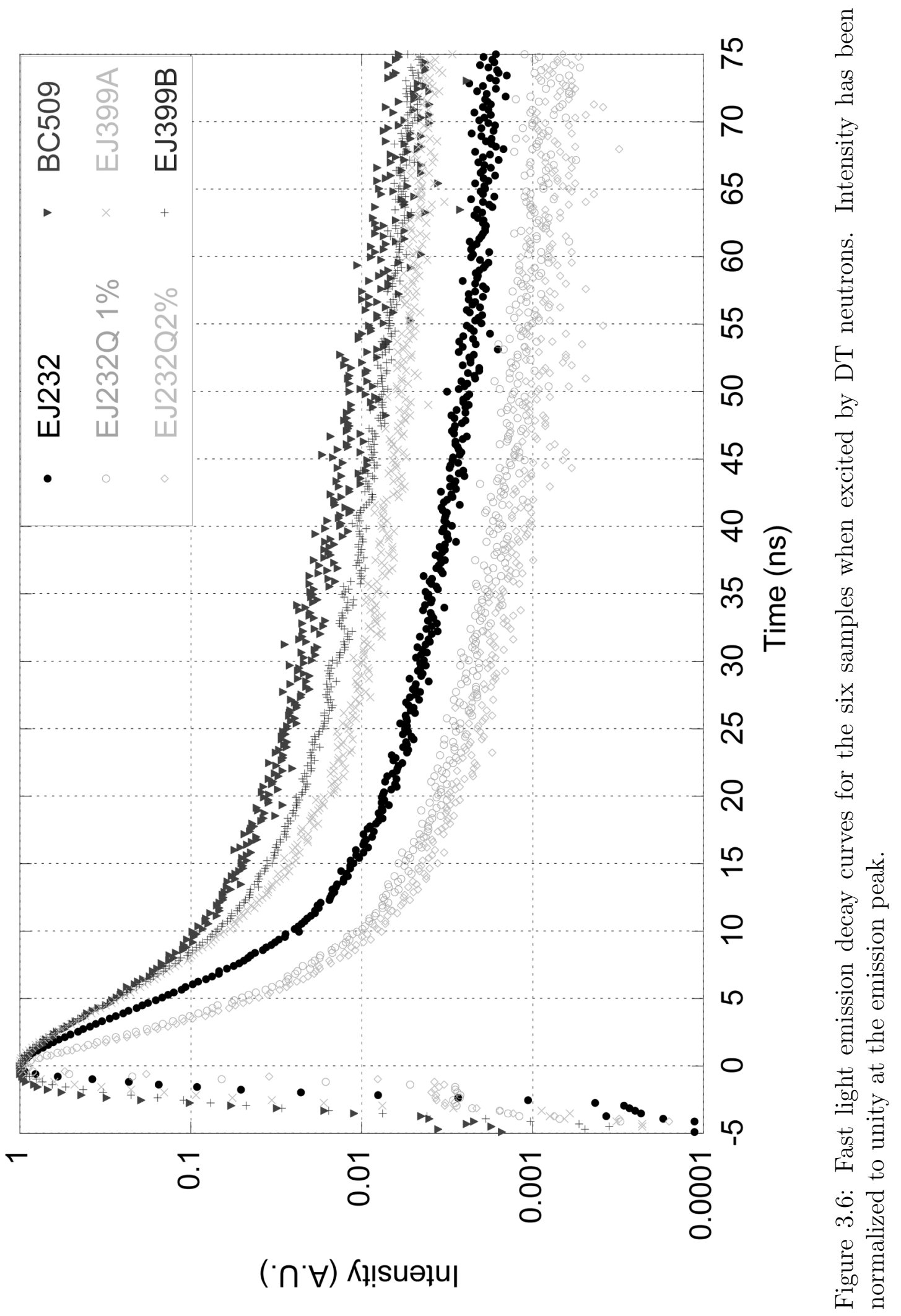




\subsection{Light Output Color Filtering}

The emission spectra of organic scintillators are broadband, having widths of up to $150 \mathrm{~nm}$, usually with a high intensity peak, with the peak wavelength quoted in table 3.1 for the studied samples. For example, the emission spectra of BC422 and BC509 are shown in figure 3.7. The broadband nature of the emitted light is due to multiple transition paths available between excited levels $\left(S_{1}, T_{1}\right)$ or sublevels $\left(S_{11}\right.$, $\left.S_{12}, S_{13} \ldots\right)$ and the ground state $\left(S_{00}\right)$ or any of its sublevels $\left(S_{01}, S_{02}, S_{03}, \ldots\right)$, as can be seen in figure 3.8. These transitions are associated with fluorescence (fast) and phosphorescence (slow) phenomena. It is reasonable to think that it could be possible to filter out the slow components of the light, keeping only the fastest transitions, and in this way reduce the afterglow contamination in the downscattered image without using a chemically quenched scintillator. The light output decay measurements
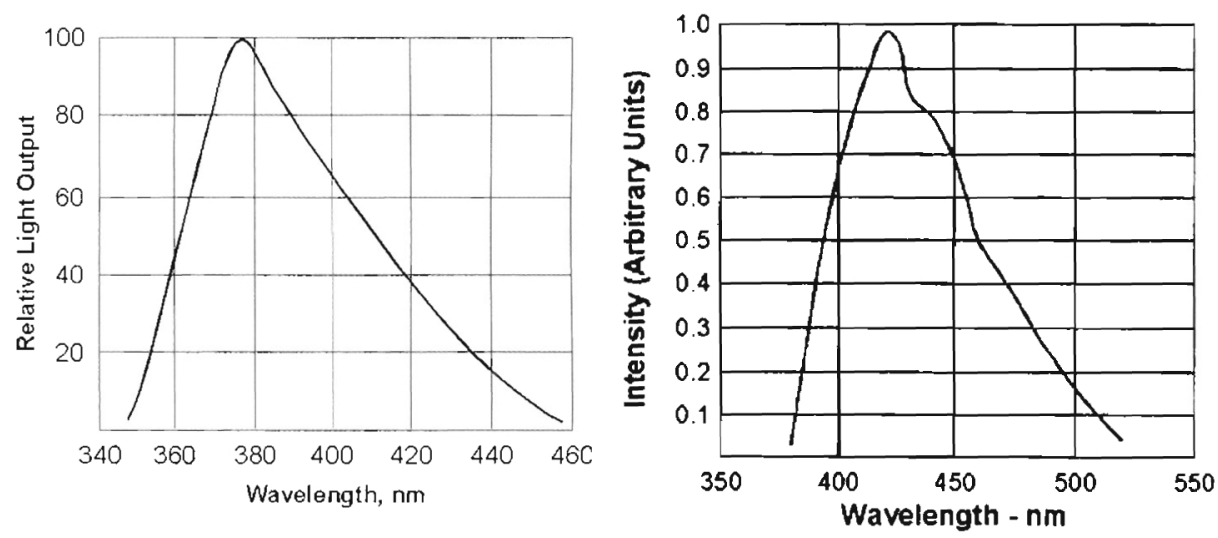

Figure 3.7: Light emission spectra for BC422 (left) and BC509 (right). Taken from Bicron's catalog. 


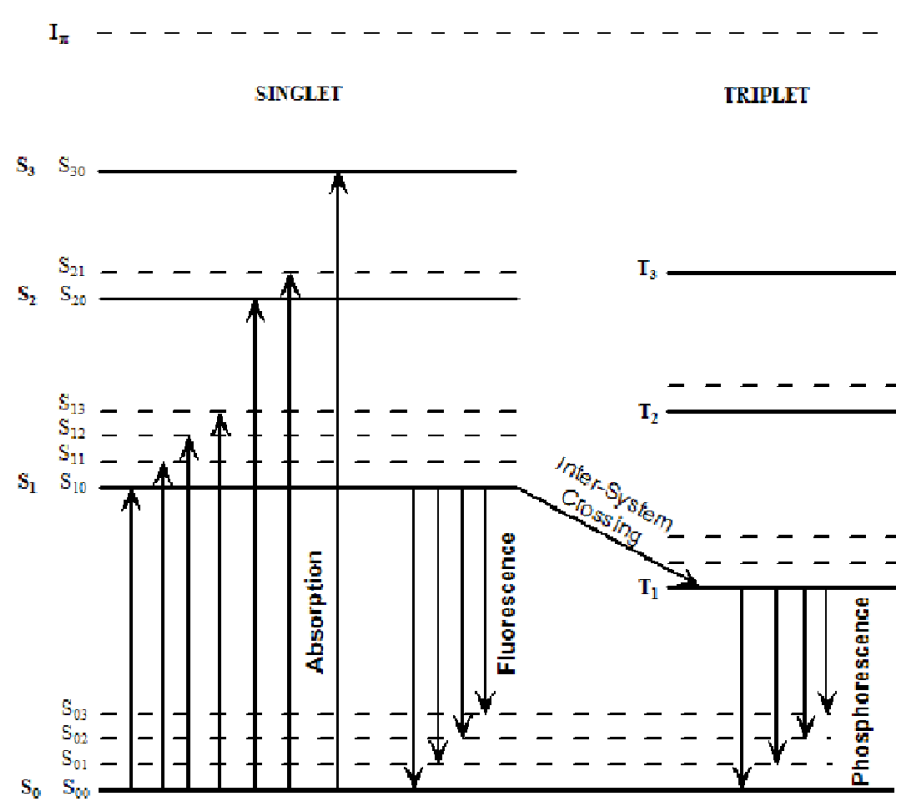

Figure 3.8: Typical level structure for the $\pi$ electrons in an aromatic molecule. $S_{0}$, ground state, $S_{1}, S_{2}, S_{3}$ excited singlet states. $T_{1}, T_{2}, T_{3}$, excited triplet states. $S_{00}$, $S_{01}, S_{02}$, etc., vibrational sublevel. $I_{\pi}, \pi$ ioniozation energy. [52]

with $14 \mathrm{MeV}$ neutrons and ${ }^{137} \mathrm{Cs}$ were repeated using a series of bandpass filters placed in front of PMT2. The filters used were Kodak Wratten gelatins and a Schott glass, the complete list is shown in table 3.3. The filters produced no observable decay component differentiation in any of the scintillators. All the filtered decay curves were equivalent to the unfiltered ones in all aspects except one, the PMT2 and coincidence count rates were significantly lower. The results for EJ399A and ${ }^{137} \mathrm{Cs}$ are shown in figure 3.9 .

The lack of decay differentiation might be due to the close proximity of the electronic levels, which translates into emission lines separated by nanometers instead of tens of nanometers. Additionally there is a known process called delayed 


\begin{tabular}{cc}
\hline \hline Filter & Band (nm) \\
& \\
\hline $34 \mathrm{~A}$ & $410-470$ \\
44 & $440-550$ \\
$47 \mathrm{~B}$ & $400-470$ \\
58 & $500-575$ \\
UG11 & $300-350$ \\
\hline \hline
\end{tabular}

Table 3.3: Band-pass filters used during the decay component differentiation studies. The top four are Kodack Wratten filters and UG11 is glass made by Schott.

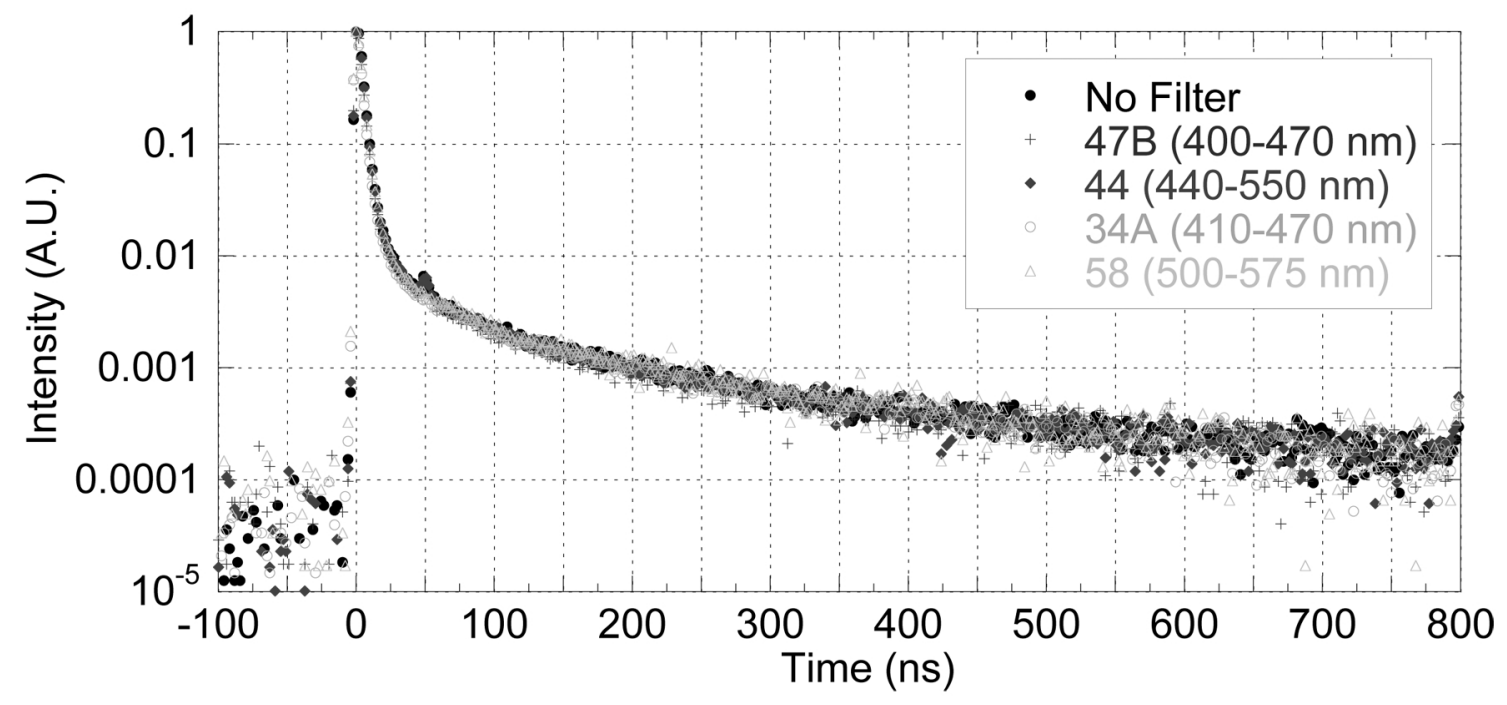

Figure 3.9: Color filtering of the light emission decay of EJ399A excited by ${ }^{137} \mathrm{Cs} \gamma-$ rays. The band-pass filters are unable to differentiate any decay components. The feature at $50 \mathrm{~ns}$ is a reflection introduced by the optic fiber bundle used in some of the measurements. 
fluorescence[56], in which a triplet state can acquire enough thermal energy and go back to a singlet state, or two triplet states can combine to form an excited singlet, a molecule in the ground state and several phonons. This process can be written as

$$
T_{10}+T_{10} \longrightarrow S_{00}+S_{1 j}+\text { phonons }
$$

The singlet state $S_{1 j}$ can decay and emit light. This process is slower than the direct singlet transition but it will produce photons of the same wavelength as the fast processes. Line filters, which are only several $\mathrm{nm}$ wide, seem the only option available to separate the decay components, but the efficiency of the system would be too low to be of practical use for the NIS, especially for downscattered imaging where a limited number of neurons is available. No further filtering studies are planned.

\subsection{Absolute Sensitivity and Photon Gain}

The modeling of the images generated by the NIS requires not only a good understanding of the neutron sources, but accurate knowledge of the scintillator light production as a function of neutron energy. The technical literature contains several studies[57][58] that explain the fundamentals of light production in organic scintillators and give plots for electrons and protons at different energies, but there are no references dealing with the calibration of the specific scintillators in this study.

The absolute light production of the six scintillators when excited by DD and DT neutrons was measured and it is expressed using two quantities: 
1. Sensitivity: photons per neutron incident. This quantity is related to the specific geometry used in the measurement; it can be used to compare the samples relative to each other.

2. Photon Gain: photons per interaction. This parameter is independent of the sample geometry and can be used for detector design.

The absolute character of these two quantities is due to the way they were measured in the laboratory. The neutron generator used is equipped with an associated particle detector (APD), which is a semiconductor detector that records the charged particle associated with a DD or DT neutron. The APD is located at a known angle from the beam line and subtends a very small solid angle; by counting the number of events on the APD, the actual neutron yield can be known with high accuracy.

Figure 3.10 shows the pulse-height spectra recorded by the APD for DD and DT. These spectra show peaks associated with the charged particles produced in the fusion reactions and are used to select the species of interest for gating and counting. For DT the alpha particle peak is selected. For DD the sharp proton peak is used instead of the wider ${ }^{3} \mathrm{He}$; at around $100 \mathrm{KeV}$ the cross sections of the two DD competing reactions are almost equal.

The electronic setup used for the absolute sensitivity measurements is illustrated in figure 3.11. Since the neutron generator output varies from one run to the other, this setup uses the number of particles counted in the APD as the reference point, instead of the actual live time or the MCA number of counts. The operation of the 

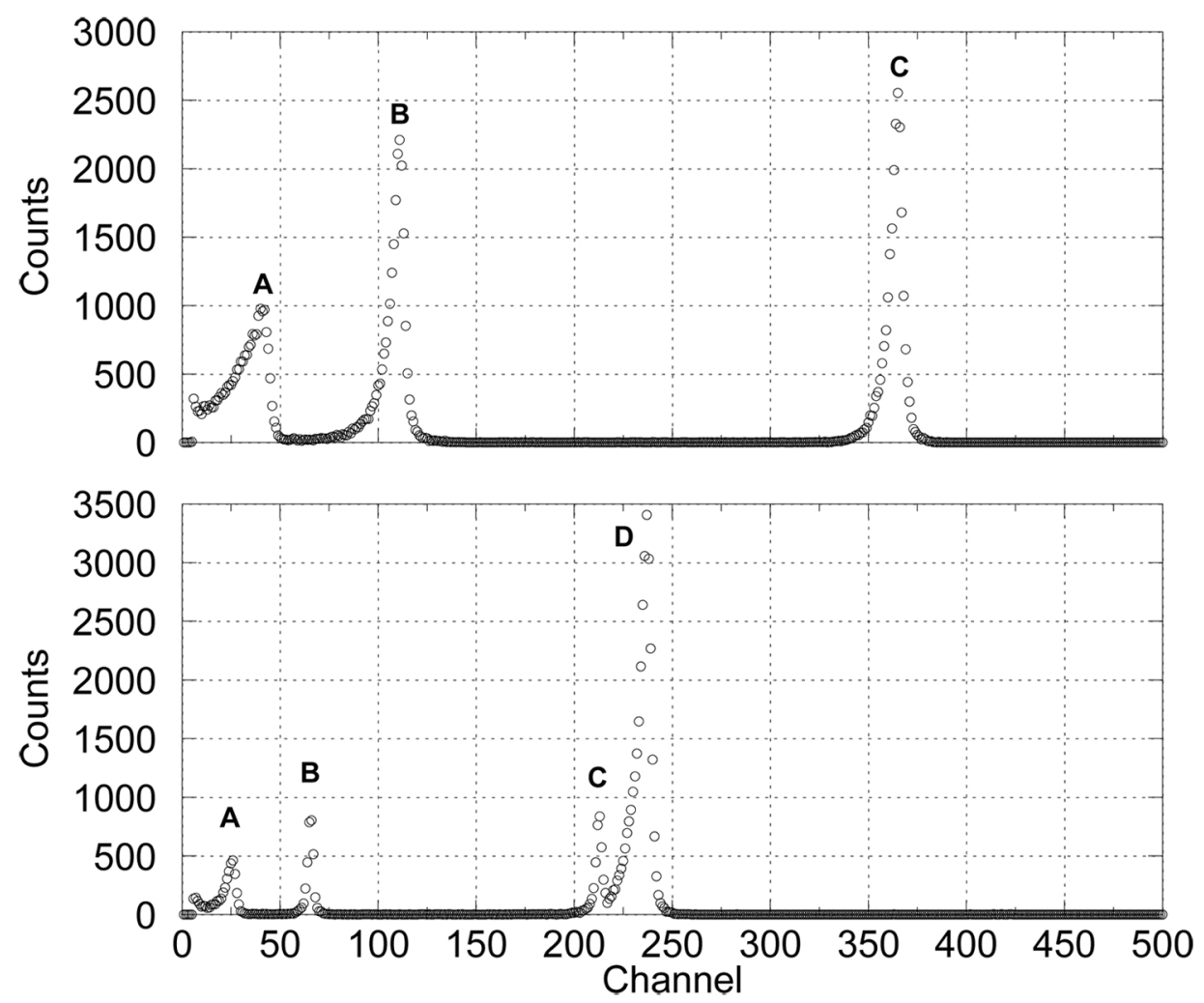

Figure 3.10: APD pulse height spectra for DD (top) and DT (bottom). The peaks correspond to: (A) $0.86 \mathrm{MeV}^{3} \mathrm{He}$, (B) $1.05 \mathrm{MeV} \mathrm{T}$, (C) $3.08 \mathrm{MeV}$ P and (D) $3.6 \mathrm{MeV}$ ${ }^{4} \mathrm{He}$. The horizontal shift is due to different values of capacitance in the preamplifier. The difference in amplitude is due to different livetimes (DD: $600 \mathrm{sec}$, DT: $210 \mathrm{sec}$ ) and the high DT cross section.

system is as follows: The species of interest in the APD is selected using the single channel analyzer (SCA). The number of desired APD events is set in the counter. The neutron generator is turned on. The MCA is activated but it only starts counting when the counter is engaged. When the number of events is reached, the MCA stops counting. The MCA counts correspond to the sum over all channels.

A new housing for the scintillator sample and the MCP-based PMT was constructed in order to increase the count rate and have a known geometry. Each sam- 


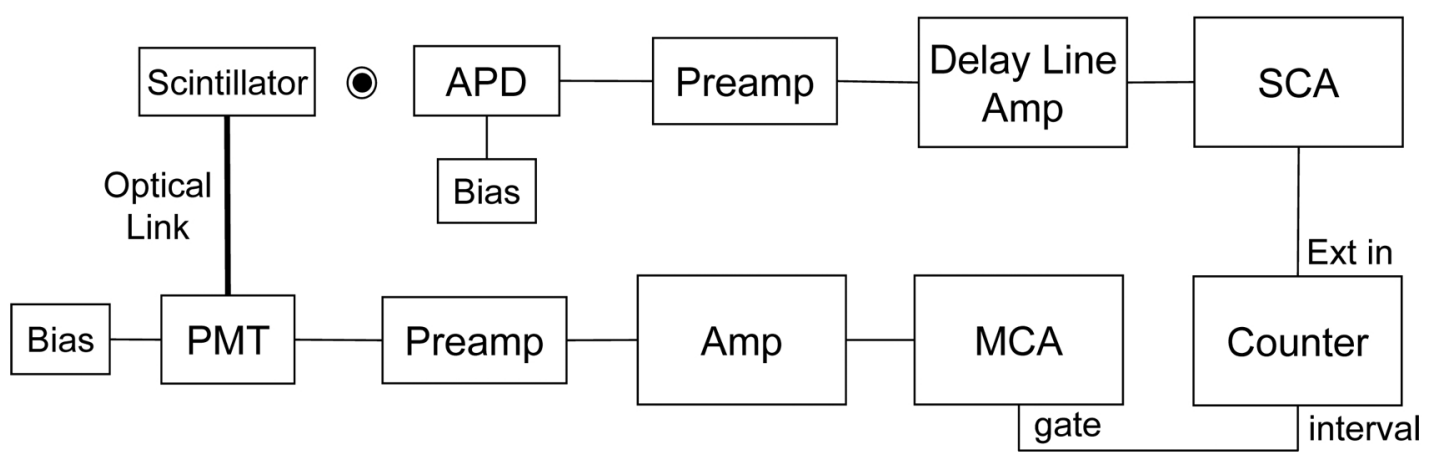

Figure 3.11: Electronic setup used in the absolute response measurements.

ple's response was measured twice, each time recording the background counts in the MCP, the number of counts in the MCA and the live time for a given number of counts in the APD. The experimental data is collected in tables 3.4 and 3.5.

The following is the calculation for EJ232 with DT neutrons. The number of counts recorded in the MCA was $1.85 \times 10^{5}$. This number needs to be corrected for non-neutron background and direct neutron interaction in the PMT. The background count rate is $24.96 \mathrm{cps}$. The direct interaction of neutrons on the PMT was separately calibrated as a function of APD count rate, and fitted to the linear function

$$
P M T_{b k g}=7.534 \times 10^{-3}\left(\frac{A P D_{\text {counts }}}{t}\right)+0.907615
$$

using $A P D_{\text {counts }}=3 \times 10^{5}$ and a live time $t=595 \mathrm{~s}, P M T_{b k g}=4.706 \mathrm{cps}$. The net MCA counts are given by

$$
M C A_{\text {net }}=1.85 \times 10^{5}-(24.96+4.706) \times 595=1.673 \times 10^{5}
$$




\begin{tabular}{|c|c|c|c|c|}
\hline Scintillator & $\begin{array}{c}\text { PMT } \\
\text { Bkgnd (cps) }\end{array}$ & $\begin{array}{c}\text { MCA } \\
\text { (counts) }\end{array}$ & $\begin{array}{c}\text { APD } \\
\left(\text { counts } \times 10^{4}\right)\end{array}$ & $\begin{array}{l}\text { Livetime } \\
\quad(\mathrm{sec})\end{array}$ \\
\hline \multirow[t]{2}{*}{ EJ232 } & 19.96 & 66451 & 3 & 847 \\
\hline & & 59896 & 3 & 602 \\
\hline \multirow[t]{2}{*}{ EJ232Q 1\% } & 18.71 & 19678 & 3 & 685 \\
\hline & & 17009 & 3 & 536 \\
\hline \multirow[t]{2}{*}{ EJ232Q 2\% } & 17.11 & 36273 & 10 & 1426 \\
\hline & & 71969 & 20 & 2828 \\
\hline \multirow[t]{2}{*}{ BC509 } & 18.01 & 20690 & 6 & 1015 \\
\hline & & 62578 & 20 & 3086 \\
\hline \multirow[t]{2}{*}{ EJ399A } & 19.42 & 47315 & 3 & 444 \\
\hline & & 75654 & 5 & 741 \\
\hline \multirow[t]{2}{*}{ EJ399B } & 17.99 & 74679 & 3 & 402 \\
\hline & & 76361 & 3 & 413 \\
\hline \multirow[t]{2}{*}{ Lucite } & 17.75 & 47447 & 20 & 2610 \\
\hline & & 78893 & 30 & 4242 \\
\hline
\end{tabular}

Table 3.4: Experimental data for DD absolute response of the six scintillator samples plus Lucite.

The number of photons from the scintillator surface is given by

$$
P h_{s s}=\frac{M C A_{n e t}}{Q E} \times \frac{1}{\Delta \Omega_{M C P}} \times \frac{1}{\Delta \Omega_{c o r}}
$$

here the first term represents the number of photons appearing on the photocathode of the PMT, where QE is the quantum efficiency at the maximum emission wavelength, in this case $Q E(\lambda=377 \mathrm{~nm})=0.1885$. The second term is the solid angle fraction of the photocathode taking the scintillator as a point source with $L=64.52 \mathrm{~cm}$ and $\phi_{P M T}=1.1 \mathrm{~cm}$

$$
\Delta \Omega_{M C P}=\frac{A}{4 \pi L^{2}}=\frac{\pi 0.55^{2}}{4 \pi 64.52^{2}}=1.8169 \times 10^{-5}
$$

and the third term is the solid angle correction due to the change in index of refraction 


\begin{tabular}{|c|c|c|c|c|}
\hline Scintillator & $\begin{array}{c}\text { PMT } \\
\text { Bkgnd (cps) }\end{array}$ & $\begin{array}{l}\text { MCA } \\
\text { (counts) }\end{array}$ & $\begin{array}{c}\text { APD } \\
\left(\text { counts } \times 10^{5}\right)\end{array}$ & $\begin{array}{l}\text { Livetime } \\
\quad(\mathrm{sec})\end{array}$ \\
\hline \multirow[t]{2}{*}{ EJ232 } & 24.96 & 126975 & 2 & 519 \\
\hline & & 185000 & 3 & 595 \\
\hline \multirow[t]{2}{*}{ EJ232Q 1\% } & 25.01 & 112746 & 9 & 1161 \\
\hline & & 55436 & 4 & 718 \\
\hline \multirow[t]{2}{*}{ EJ232Q 2\% } & 24.78 & 31235 & 4 & 539 \\
\hline & & 144194 & 20 & 2134 \\
\hline \multirow[t]{2}{*}{ BC422 } & 26.12 & 188763 & 3 & 498 \\
\hline & & 375915 & 6 & 919 \\
\hline \multirow[t]{2}{*}{ BC422Q 1\% } & 24.95 & 59063 & 5 & 610 \\
\hline & & 111454 & 10 & 903 \\
\hline \multirow[t]{2}{*}{ BC422Q 2\% } & 24.80 & 38120 & 5 & 638 \\
\hline & & 72265 & 10 & 1151 \\
\hline \multirow[t]{2}{*}{ BC509 } & 26.43 & 63994 & 8 & 895 \\
\hline & & 174134 & 20 & 2706 \\
\hline \multirow[t]{2}{*}{ EJ399A } & 26.87 & 112076 & 2 & 1051 \\
\hline & & 157193 & 3 & 1195 \\
\hline \multirow[t]{2}{*}{ EJ399B } & 25.63 & 158340 & 2 & 738 \\
\hline & & 232246 & 3 & 893 \\
\hline \multirow[t]{2}{*}{ Lucite } & 23.78 & 37743 & 9 & 1239 \\
\hline & & 72732 & 20 & 2294 \\
\hline
\end{tabular}

Table 3.5: Experimental data for DT absolute response of the six scintillator samples plus Lucite and the three Bicron 422.

$$
\left(n_{\text {plastic }}=1.58, n_{\text {air }}=1\right)
$$

$$
\Delta \Omega_{\text {cor }}=\left(\frac{1}{n_{\text {plastic }}}\right)^{2}=0.401
$$

using 3.5 and 3.6 in 3.4

$$
P h_{s s}=\frac{1.673 \times 10^{5}}{0.1885} \times \frac{1}{1.8169 \times 10^{-5}} \times \frac{1}{0.401}=1.22 \times 10^{11}
$$

The net number of photons produced in the scintillator $P h_{n e t}$, assuming that they all 


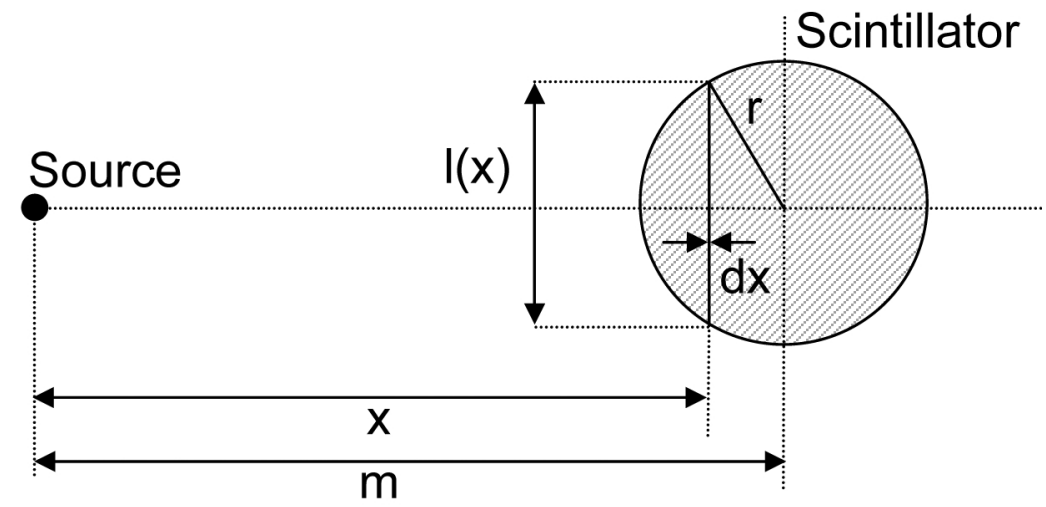

Figure 3.12: Geometry construct used for the calculation of the average scintillator solid angle fraction $\overline{\Delta \Omega}_{s c i}$.

escape through the face oriented toward the PMT, is given by

$$
P h_{n e t}=P h_{s s} \times \frac{1}{1-\left(\frac{n_{\text {plastic }}-1}{n_{\text {plastic }}+1}\right)^{2}}=1.285 \times 10^{11}
$$

where the second term is related to the refraction losses in the plastic-air interface.

Using the APD solid angle fraction $\Delta \Omega_{A P D}=7 \times 10^{-5}$ and $3 \times 10^{5}$ counts, the total number of neutrons produced, assuming an isotropic distribution, is given by

$$
N_{t o t}=3 \times 10^{5} \times \frac{1}{\Delta \Omega_{A P D}}=4.286 \times 10^{9}
$$

of these the ones entering the scintillator are given by

$$
N_{s c i}=N_{t o t} \times \overline{\Delta \Omega}_{s c i}
$$

The scintillator average solid angle fraction $\overline{\Delta \Omega}_{s c i}$ can be calculated using the expression for the average cross sectional area of a cylinder (see figure 3.12)

$$
A(x)=H \times l(x)
$$




$$
l(x)=2 \sqrt{r^{2}-(m-x)^{2}}
$$

using the same approximation for the solid angle fraction as in 3.5 and integrating across the cylinder

$$
\overline{\Delta \Omega}_{s c i}=\frac{A(x)}{4 \pi x^{2}}=\frac{\int_{m-r}^{m+r} \frac{2 H \sqrt{r^{2}-(m-x)^{2}}}{4 \pi x^{2}} \mathrm{~d} x}{2 r}
$$

where $H$ is the cylinder's height, $r$ its radius and $m$ is the distance between the neutron source and the center of the sample. Using $H=3.81 \mathrm{~cm}, r=1.905 \mathrm{~cm}$, $m=11.43 \mathrm{~cm}$, and integrating numerically gives $\overline{\Delta \Omega}_{s c i}=7.09262 \times 10^{-3}$, inserting into 3.10

$$
N_{s c i}=4.286 \times 10^{9} \times 7.09262 \times 10^{-3}=3.04 \times 10^{7}
$$

The absolute sensitivity is then given by

$$
\text { Sensitivity }=\frac{P h_{\text {net }}}{N_{s c i}}=\frac{1.285 \times 10^{11}}{3.04 \times 10^{7}}=4227 \frac{\text { photons }}{\text { neutron incident }}
$$

The photon gain can be calculated using the expression

$$
\text { Photon Gain }=\frac{P h_{\text {net }}}{\text { int }}
$$

where the number of neutron interactions int is given by

$$
\text { int }=N_{s c i} \times(N \sigma \bar{x})
$$

the term in parenthesis is the neutron interaction probability, where $N$ is the atomic density, $\sigma$ is the nuclear cross section and $\bar{x}$ is the average distance traversed by the neutron inside the scintillator. $N$ and $\sigma$ are related to the predominant light 
production mechanism. Up to $12 \mathrm{MeV}$ the most effective mechanism is proton recoil, above this energy, carbon recoil and alpha breakup start contributing[34]. In this study only proton recoil has been considered, even at $14 \mathrm{MeV}$. This means that $N=N_{H}$ and $\sigma$ is the proton elastic scattering cross section. In the case of BC509 the number of interactions was calculated taking into account hydrogen, carbon and fluorine $^{4}$. It is not clear which process is responsible for most of the light production in this case.

Instead of calculating $\bar{x}$ by hand and carrying out the multiplication shown in 3.17, the whole factor int can be computed by running an MCNPX (see chapter four) simulation of the actual sample geometry with the exact number of DT neutrons $\left(N_{t o t}\right)$, and taking the composition as pure hydrogen gas at a density that matches the atomic concentration

$$
5.19 \times 10^{22} \frac{\text { atoms }}{\mathrm{cm}^{3}} \Leftrightarrow 8.6184 \times 10^{-2} \frac{\mathrm{g}}{\mathrm{cm}^{3}}
$$

The MCNPX output gives the number of protons created by elastic scattering of the $14 \mathrm{MeV}$ neutrons, regardless of their energy; this number is equivalent to int. For BC509 the actual composition was used and all the neutron interactions were taken into account. For the sample calculation this gives $i n t=3837262$ and 3.16 becomes

$$
\text { Photon Gain }=\frac{1.285 \times 10^{11}}{3.84 \times 10^{6}}=33480 \frac{\text { photons }}{\text { interaction }}
$$

in a final step the results of the two measurements are averaged.

\footnotetext{
${ }^{4}$ The actual atomic concentrations of BC509 are: C: $3.16 \times 10^{22}$, H: $0.011 \times 10^{22}$ and F: $3.15 \times 10^{22}$ atoms per $\mathrm{cm}^{3}$. Data taken from Bicron's catalog.
} 
The calculation for DD neutrons follows the same steps, except that the PMT background correction obeys a different linear equation, and the distance from the sample to the PMT $L$ was reduced to $49.98 \mathrm{~cm}$, and the distance from the scintillator to the neutron source $m$ was $6.35 \mathrm{~cm}$.

The absolute sensitivity and photon gain results are collected in table 3.6. The sensitivity can be used to easily compare the scintillator performance, table 3.7 shows the sensitivity relative to EJ232 with DT neutrons. The accuracy of these results can be verified by looking at the values of light output as a percentage of the anthracene response given by the manufacturers; for EJ232, EJ232Q 1\% and EJ232Q 2\% the corresponding values are 55\%,11\% and 5\%, which correspond to $1.00,0.20$ and 0.09 in the scale used in table 3.7. The experimental values are up to $33 \%$ lower, but in the same ballpark.

Another interesting comparison can be made using the measured response and light output of BC400, a plastic scintillator also known as NE102, that is similar to BC422 (EJ232). Figure 3.13 shows the relative response measured by Smith et.al.[57] and figure 3.14 shows the number of scintillation photons produced vs. particle energy as reported in Bicron's catalog[59].

The relative response curve shows that both the DD and DT measurements fall on the linear portion of the curve, i.e., light emission is directly proportional to the $\mathrm{d} E / \mathrm{d} x$ of the recoil proton, but using $7.5 \mathrm{MeV}$ and $1.25 \mathrm{MeV}$, the average recoil proton energies for DT and DD neutrons respectively, the relative response ratio is 


\begin{tabular}{lcccc}
\hline \hline Sample & $\begin{array}{c}\text { DD } \\
\text { Sensitivity }\end{array}$ & $\begin{array}{c}\text { DD } \\
\text { Photon Gain }\end{array}$ & $\begin{array}{c}\text { DT } \\
\text { Sensitivity }\end{array}$ & $\begin{array}{c}\text { DT } \\
\text { Photon Gain }\end{array}$ \\
\hline EJ232 & 2200 & 7582 & 4236 & 33565 \\
EJ232Q 1\% & 312 & 1075 & 640 & 5073 \\
EJ232Q 2\% & 118 & 406 & 277 & 2195 \\
BC422 & NM & NM & 4373 & 34688 \\
BC422Q 1\% & NM & NM & 604 & 4795 \\
BC422Q 2\% & NM & NM & 269 & 2134 \\
BC509 & 19 & 40 & 252 & 797 \\
EJ399A & 1774 & 6183 & 3322 & 26639 \\
EJ399B & 3033 & 10606 & 5274 & 42499 \\
Lucite & NM & NM & 3 & 22 \\
\hline \hline
\end{tabular}

Table 3.6: Scintillator sensitivity and photon gain results. NM: not measured.

\begin{tabular}{lcc}
\hline \hline Sample & 2.5 $\mathbf{M e V}$ neutrons & $\mathbf{1 4} \mathbf{~ M e V}$ neutrons \\
& & \\
\hline EJ232 & 0.5195 & 1.0000 \\
EJ232Q 1\% & 0.0737 & 0.1510 \\
EJ232Q 2\% & 0.0279 & 0.0654 \\
BC422 & $\mathrm{NM}$ & 1.0323 \\
BC422Q 1\% & $\mathrm{NM}$ & 0.1427 \\
BC422Q 2\% & $\mathrm{NM}$ & 0.0635 \\
BC509 & 0.0045 & 0.0595 \\
EJ399A & 0.4189 & 0.7843 \\
EJ399B & 0.7160 & 1.2452 \\
Lucite & $\mathrm{NM}$ & 0.0007 \\
\hline \hline
\end{tabular}

Table 3.7: Scintillator sensitivity relative to EJ232 excited by DT neutrons. NM: not measured. 


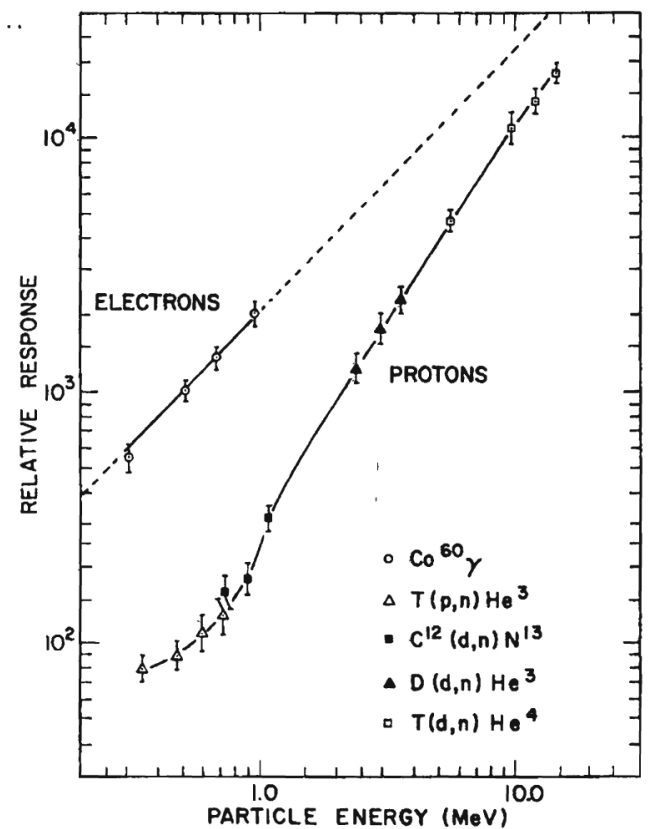

Figure 3.13: Relative response of NE102 (equivalent to BC400) as reported by Smith et.al.[57].

0.11 instead of the measured 0.51. This discrepancy can be explained by looking at the way both measurements were made. Smith et.al. used different tunable sources to construct the curve point by point, in this particular case the recoil protons were produced by DT neutrons and then a single energy was selected. The measurement presented in this study used the whole spectrum of recoil protons, from $14 \mathrm{MeV}$ to zero for DT neutrons. This broad proton spectrum gives rise to a higher response.

Figure 3.14 shows that a $7.5 \mathrm{MeV}$ proton produces around $3 \times 10^{4}$ photons, but a $1.25 \mathrm{MeV}$ proton only generates $2 \times 10^{3}$, this is 15 times less. The ratio between the experimental values for EJ232 is only 4.42. This huge discrepancy has to do, not only with the nature of the measurement, and the fact that BC400 has a $65 \%$ 


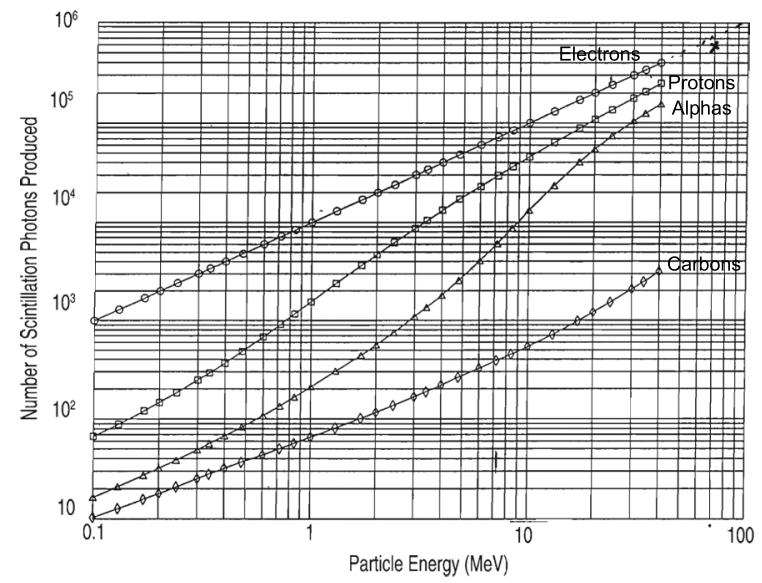

Figure 3.14: Light production curves for plastic scintillator BC400. Taken from Bicron's general catalog

anthracene light output (55\% for EJ232), but with the definition of photon gain in this study. Since MCNPX counts all the protons created by elastic recoil, regardless of their energy, the number of light producing interactions int is not the same as the monoenergetic proton used in the plot. MCNPX accurately accounts for the increase of the elastic scattering cross section at lower neutron energies, but it does not say anything about the proton $\mathrm{d} E / \mathrm{d} x$ that becomes higher at lower energies.

In the case of BC509 the inaccuracies in the results might be enhanced by the fact that all the components were used instead of just hydrogen, this translates into lower values of photon gain. Although there are no light production plots available for comparison, BC509 is reported to have a light output of $20 \%$ anthracene $(0.36)$, which is much higher than the measured 0.06 .

An additional sensitivity measurement at an intermediate energy is necessary to accurately model the response of the scintillator. A tunable neutron source is required 
since the neutron generator at LLNL can not provide other energies.

Regarding the comparison between manufacturers, the $14 \mathrm{MeV}$ results for BC422, BC422Q 1\% and BC422Q 2\% are between experimental error from those of their Eljen counterparts, so the formulations can be considered equivalent. Also for this series, the relative sensitivity results make evident the great impact that chemical quenching has on light production; EJ232Q 1\% emits 15\% of the light of EJ232. The $2 \%$ version emits only $6.5 \%$ and, as was shown in the previous section, the late-time decay improvement was minimal.

A sample of pure Lucite ${ }^{5}$ was included in the study as a system check; Lucite does not scintillate; it only emits light in the form of to Cherenkov radiation, which is an instantaneous and very inefficient process. The instantaneous nature was used to verify the light emission decay, and the low efficiency to see how sensitive the photon counting system was.

\subsection{Fiber Array Light Emission Distribution}

Although the detector-optical system design has not been finalized, the possibility of recording multiple simultaneous images is very interesting. Bicron produces several plastic scintillators in fiber form, these are step-index fibers that act as wave guides. Lerche et.al.[60] and Christensen et.al.[47] have used fiber arrays in their experiments,

\footnotetext{
${ }^{5}$ Lucite is the commercial name of Poly methyl-methacrylate PMMA. C: $3.583 \times 10^{22}$, H: $5.733 \times$ $10^{22}$ and O: $1.433 \times 10^{22}$ atoms per $\mathrm{cm}^{3} . \mathrm{n}=1.47 \rho=1.19 \mathrm{~g} / \mathrm{cm}^{3}$.
} 
but the fibers were not very regular and had a coating on the outside to enhance reflection, which made construction more complex. Bicron claims they can produce better quality fibers now and that these fibers have a numerical aperture (NA) of 0.58, which would allow placing cameras at large angles from the line-of-sight axis.

A small $10 \times 10$ array was built at LLNL in order to measure its light emission angular distribution and verify the big NA claim. The fibers used were BCF-20, 1 $\mathrm{mm}$ in diameter, $25.4 \mathrm{~mm}$ in length, and arranged in a square pitch pattern. The scintillator housing used in the bulk sample measurements was modified to allow the movement of the PMT. The same electronic setup (see figure 3.11)was used but only DT neutrons were measured. The experimental data is listed in table 3.8 .

The relative sensitivity calculations follow the same algorithm as before, the number of APD counts was constant $\left(6 \times 10^{5}\right)$, and a new MCNPX simulation was used to compute the number of neutrons absorbed in the fibers, accounting for the actual geometry and composition.

The results relative to the on-axis value are shown in figure 3.15. There is a slow decline in light emission as the viewing angle passes 25 degrees, in fact there is still plenty of light present even at 40 degrees, which should provide enough space to field multiple optical systems. The relative sensitivity between 5 and 20 degrees shows values greater than unity. At this time there is no complete explanation, it might be due to experimental error or some plastic-air interface refraction effect. Measurements should be repeated using BC422 square cross section fibers and a bigger array, but in 


\begin{tabular}{cccc}
\hline \hline $\begin{array}{c}\text { Angle } \\
\text { (degrees) }\end{array}$ & $\begin{array}{c}\text { Live Time } \\
\text { (sec) }\end{array}$ & $\begin{array}{c}\text { MCA } \\
\text { (counts) }\end{array}$ & $\begin{array}{c}\text { Background } \\
\text { (cps) }\end{array}$ \\
\hline 0 & 867 & 44955 & 23.32 \\
5 & 859 & 46179 & 23.32 \\
5 & 856 & 45759 & 23.32 \\
10 & 858 & 45496 & 23.32 \\
10 & 848 & 45581 & 23.32 \\
15 & 890 & 45972 & 23.32 \\
15 & 838 & 45722 & 23.32 \\
20 & 810 & 44383 & 23.32 \\
20 & 863 & 45135 & 23.32 \\
25 & 884 & 44064 & 23.32 \\
25 & 873 & 44331 & 23.32 \\
30 & 889 & 43195 & 23.66 \\
30 & 903 & 44183 & 23.66 \\
35 & 940 & 43210 & 23.66 \\
35 & 884 & 41988 & 23.66 \\
40 & 791 & 38721 & 23.66 \\
40 & 803 & 39606 & 23.66 \\
45 & 830 & 38039 & 23.66 \\
45 & 855 & 38687 & 23.66 \\
\hline \hline
\end{tabular}

Table 3.8: Experimental data for the light emission angluar distribution of the plastic fiber array. The quoted live times are relative to $6 \times 10^{5}$ counts in the APD.

general these results are very promising.

\subsection{Scintillator Selection}

Based on the light decay profile and the absolute sensitivity measurements, the best candidates for the NIS detector are the plastic EJ232 (BC422) and the liquid EJ399B. EJ232 has a fast decay profile and good photon gain, and it is available in 


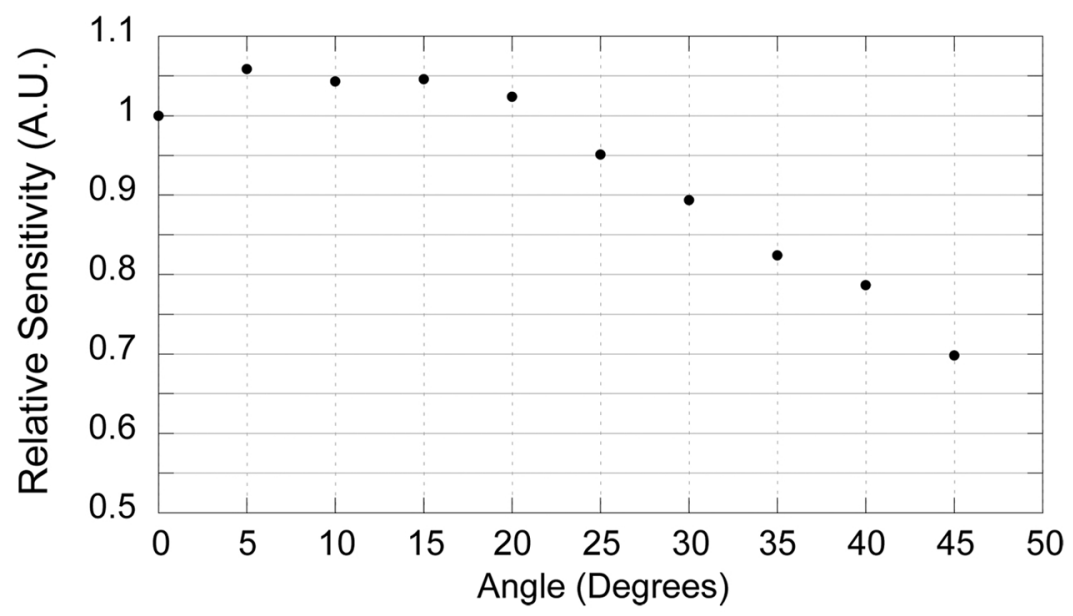

Figure 3.15: Light output distribution, in terms of relative sensitivity, for a $10 \times 10$ array of plastic fibers excited by $14 \mathrm{MeV}$ neutrons.

square cross section step-index fibers. EJ399B has a slower decay profile but shows an excellent photon gain, 24\% higher than EJ232. Both of these scintillators are long lived and will not produce an afterglow-free downscatted image. An image subtraction algorithm is required, as it will be shown in the next chapter.

BC509 shows a steeper decay profile than the other samples, which is good for the image generation and subtraction, but it has a very low photon gain, which places a big burden on the recording statistics. The main feature of BC509 was its hydrogen free chemistry, which is thought to translate in higher spatial resolution.

The promising light distribution results of the small fiber array are the basis for an innovative optical system design currently being considered at LLNL, where multiple cameras will image the back face of the scintillator array while being out of the neutron line-of-sight. 


\section{Chapter 4}

\section{Model-based Neutron Imaging}

Before building a prototype of the neutron imaging system for the NIF it is important to understand the kinds of iamges that the system will record, and how these recorded iamges can be used to satisfy the pre-established requirements. This chapter introduces the 'End-to-End Model' of the NIS; a simulation tool that uses a simulated neutron source distribution as input, and then produces a recorded image as output. This chapter also presents an algorithm for the formation of the primary and downscattered images, explicitly taking into account the scintillator decay presented in chapter three. As part of the system response characterization sources such as points, flat discs or slabs, are used to study the morphology of the recorded images that are produced by model. 


\subsection{The End-to-End Model}

The different components of the NIS can be studied individually, as was done with the scintillators in the previous chapter. However, since the ultimate goal of this study is to quantify the performance of the whole system, it makes sense to model it as a complete unit. Figure 4.1 illustrates the End-to-End model that fullfills this purpose.

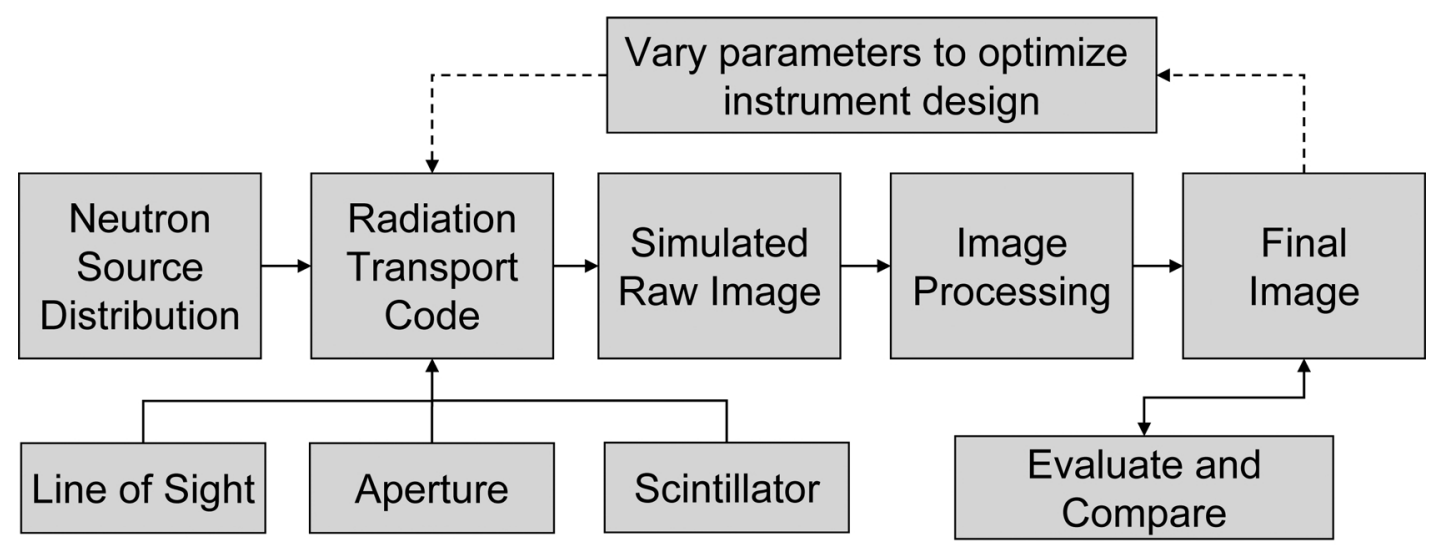

Figure 4.1: Block diagram of the End-to-End model for the NIS.

The model extends from a source distribution located at TCC to the photocathode of a generic optical system. The model's input is a detailed neutron source distribution which is used by a radiation transport code that contains the necessary details about the geometry and location of the aperture, the choice of scintillator and the location and geometry of the detector. The code transports the neutrons through the aperture and into the pixelated scintillator, where they interact with the carbon and hydrogen atoms, producing recoil protons, whose energy deposition is tallied as MeV per pixel. 
An image formation algorithm transforms this information into photo-electrons per pixel, producing a good measure of the raw recorded image intensity and statistics. Some post-processing is required in order to obtain a useful neutron image, which in turn is compared to the original source distribution. The model parameters can be adjusted to optimize a particular feature, and the process is repeated until the results are satisfactory.

\subsection{Neutron Source Distributions}

The sources used in this study were produced by detailed hydrodynamic simulations of failed NIF-scale implosions. The fizzle studied was caused by a pure $P_{6}$ driver asymmetry. The code generates a $20 \times 20$ array of 2.5 micron square elements, covering one fourth of the source plane. The complete source is obtained by assuming fourfold symmetry, producing a square FoV of 100 microns. This 2D array is a representation of a 3D source, and was generated taking into account the kinematics described in section 2.1. The energy spectrum of the neutrons that escape from the compressed target is divided into nine one-MeV bins, from 6 to $15 \mathrm{MeV}$. The value of each source element represents the number of neutrons emitted in that energy bin per steradian, per unit area $\left(\mathrm{cm}^{2}\right)$ and per primary neutron; this allows the use of the same source set at different primary yields and with arbitrary size detectors located anywhere. It is important to note that these values have zero noise and perfect resolution; these arrays should not be considered realistic images. For simplicity each 
energy bin is assumed monoenergetic and with $E_{n}$ set to the middle of the interval, that is $6.5,7.5$, etc. The distribution set is valid for primary yields between $5 \times 10^{15}$ and $2 \times 10^{17}$. This study uses the minimum downscattered yield required for the NIS, i.e., $5 \times 10^{14}$, which translates into $6.83 \times 10^{15}$ primary neutrons.

Figure 4.2 shows the nine $40 \times 40$ pixels source distributions in a 256 level grayscale, where each image has been normalized to its maximum (white). The plot at the bottom shows the emitted neutron spectrum, in total neutrons per energy bin, at the minimum downscattered yield. The integrated primary (13-15 MeV) and downscattered (6-10 MeV) sources are shown in figure 2.6.

\subsection{MCNPX}

The core of the End-to-End model is the radiation transport code. Its main two tasks are to sample a given source distribution and transport it through the high-Z aperture. Since the only important nuclear process occurring in the aperture is elastic scattering, the transport could have been accomplished with the use of a ray-tracing code that included the intensity attenuation. The model also includes the scintillator array, accounting for the finite range of the recoil protons in the pixels, and tallying their energy depositions. These tasks were beyond the scope of a simple raytracing code. The solution to this problem was to use an existing radiation transport code: Monte Carlo Neutron Photon eXtended (MCNPX 2.5.0)[61]. This code is based on the older MCNP4C, it can track charged particles like protons, alphas and 

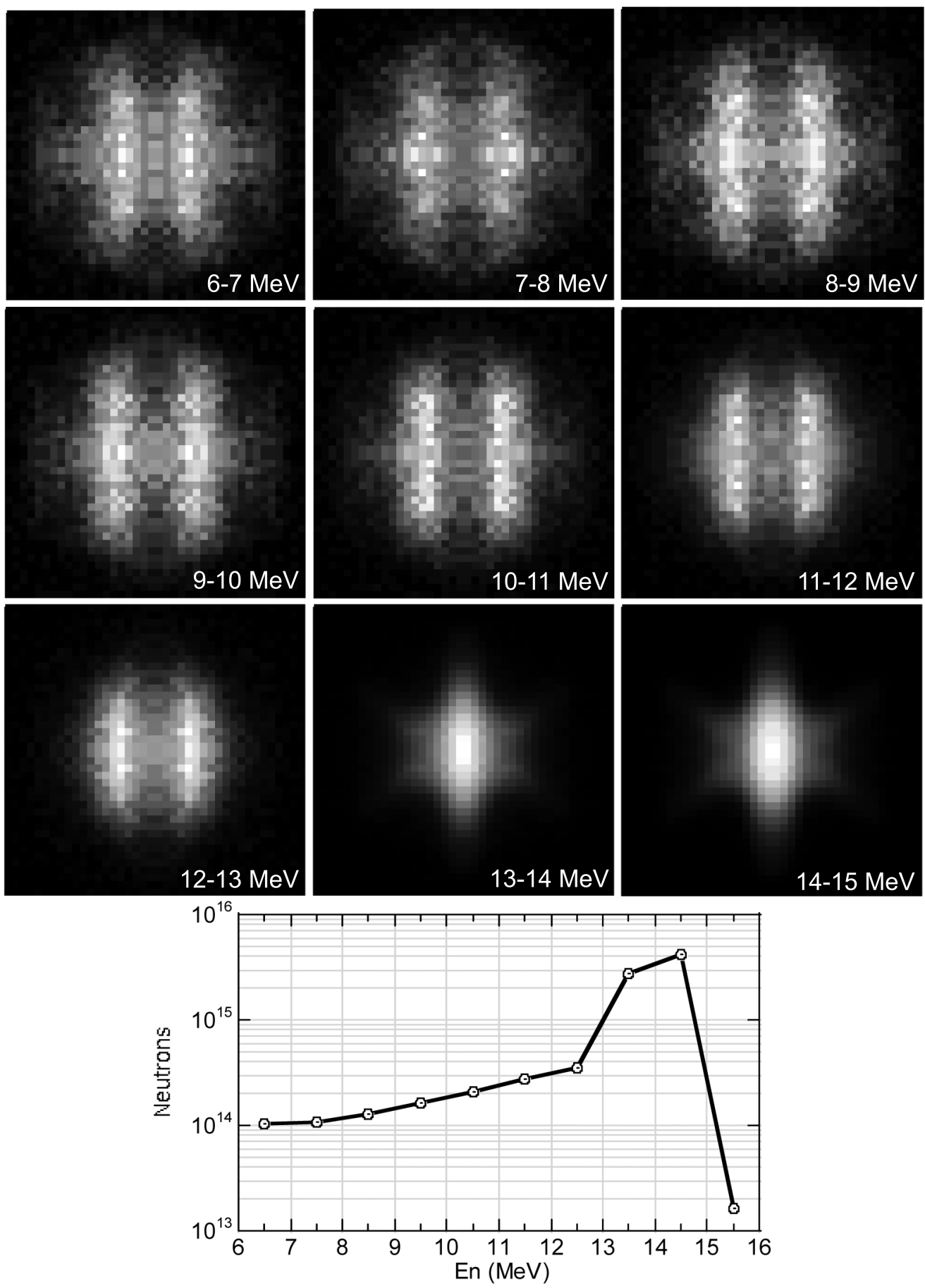

Figure 4.2: Simulated neutron source distributions for a fizzle caused by a $P_{6}$ driver asymmetry. Each image is normalized to its maximum intensity (white). Bottom: Emitted neutron spectrum for the minimum required downscattered yield of $5 \times 10^{14}$, each bin is assumed monoenergetic and centered in the enegy interval. Simulation by S. Hatchett. 
deuterons. MCNPX, which is developed and maintained by the Los Alamos National Laboratory and distributed through the Radiation Safety Information Computational Center (RSICC) of the Oak Ridge National Laboratory ${ }^{1}$, is widely used in research and industry, and it is considered a standard for radiation transport calculations.

The NIS MCNPX line-of-sight model consists of a source located at TCC, a high-Z aperture located $40 \mathrm{~cm}$ away, and a pixelated scintillator detector located either 28 or $40 \mathrm{~m}$ from the aperture. No additional structural elements, like the target chamber, aperture fixture or the detector optical system, are included. The components are assumed to be in a vacuum.

\subsubsection{Preliminary Simulations}

A series of partial simulations was completed before performing the whole system calculations. The goal was to understand the effects on the image and verify the way MCNPX deals with two particular processes: the neutron scattering inside the high-Z aperture and the proton energy deposition in the pixelated detector. These processes have a negative impact on the CPU tim and the NIS resolution. Since there are several ways to implement a calculation in MCNPX, it was also important to identify the method which produced the most accurate results.

\footnotetext{
${ }^{1}$ MCNPX webpage: monpx.lanl.gov RSICC webpage: www-rsicc.ornl.gov/index.html
} 


\subsubsection{Neutron Scattering in the Aperture}

When simulated neutrons go through the high-Z aperture only a very small fraction goes through the open pinhole, or traverses the material uncollided. With normal settings, MCNPX tracks each neutron through its collisions until it is either captured or escapes from the geometry. Since neutrons that scatter in the apperture have a very small probability of reaching the detector, the model saves a great deal of time by tracking only the uncollided neutrons.

Two simple simulations were performed in order to quantify the contribution of the scattered neutrons to the image, and to measure the computing time. An $8 \mathrm{MeV}$ neutron point source partially illuminates a solid block of tungsten $13.7 \mathrm{~cm}$ thick $40 \mathrm{~cm}$ away, while a small portion of the beam is unobstructed. The neutrons are generated inside a narrow cone that covers the central region of the detector, which is located $40 \mathrm{~m}$ away, and is made up of $128 \times 128250$ micron square pixels, $1 \mathrm{~mm}$ thick. No scintillator is included. $10^{9}$ particles are generated in each run, in the first one the neutron energy cut-off is zero, while in the second one it is $7.999 \mathrm{MeV}$.

Figure 4.3 shows the arrays obtained from both runs. The left image corresponds to zero cut-off, the right one to $7.999 \mathrm{MeV}$. They are shown in a 256 level grey scale with an upper level threshold to enhance the small values. Individual unit valued pixels have been artificially enhanced so they are noticeable. Both images show a random pattern of scattered neutron counts outside the source cone. Figure 4.3 also shows a vertical lineout through the center of both images. The plot clearly shows 

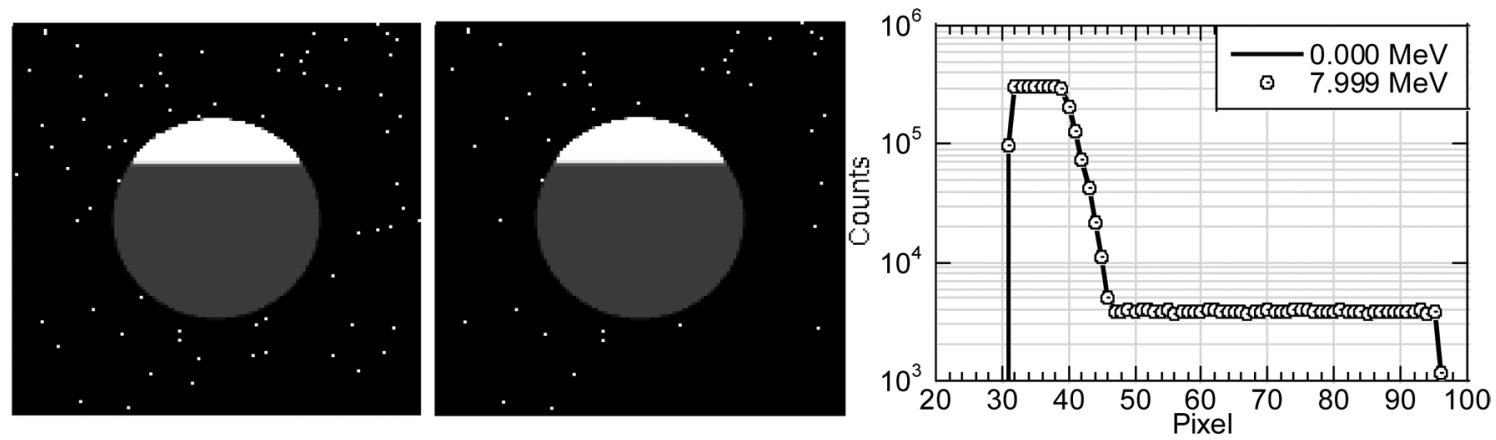

Figure 4.3: Simulated images showing the effect of neutron energy cut-off in the scattered counts. No cut-off (left), $7.999 \mathrm{MeV}$ cut-off (center). The plot shows that the neutron attenuation of the solid $13.7 \mathrm{~cm}$ thick tungsten block is the same in both cases.

the attenuation effect of the tungsten block, almost $1 \%$ direct transmission for 13.7 $\mathrm{cm}$. The difference in total detected counts between the two cases is only 39 counts, but the CPU time is almost $60 \%$ shorter for the $7.999 \mathrm{MeV}$ cut-off case.

These simulations show that the effect on the image of the neutrons scattered in the aperture is negligible, and that a $1 \mathrm{keV}$ energy loss is enough to take the neutron out of the problem without compromising the accuracy of the model. A neutron energy cut-off card will be included in all calculations.

\subsubsection{Scintillator Energy Deposition}

When an energetic neutron enters the scintillator material, the multiple nuclear processes that can occur include elastic scattering from carbon or hydrogen, ionization, carbon breakup into alpha particles, and atomic excitation followed by the release of one or several photons. MCNPX can keep track of all of these processes 
and account for the energy deposited by each particle. The more particles the code tracks, the more complex and slower it gets. It is necessary to find a balance between complexity and accuracy.

MCNPX has two types of energy deposition tallies: f6:x and +f6. The first tracks only one type of particle, $\mathrm{x}$, like neutrons (n), photons (p) or protons (h). The second considers all the particles that are enabled by the mode card in the input deck, and generates a global value of deposited energy. Two test simulations showed that the contributions of electrons and photons translated into a flat background, due to their long mean free paths, and that the contribution from heavy charged particles, like alphas and carbon ions, was very small at low energies and that their range was too short to impact the resolution.

Several tests where done using the $+\mathrm{f} 6$ tally with neutrons and protons, but the deposited energy distributions generated had extremely high values in the first few microns and long ionization tracks. This seems to be due to MCNPX dumping all the energy of heavy charge particles right at the place of interaction and accounting for some additional nuclear reactions, the manual is not very clear on how the $+\mathrm{f} 6$ energy deposition is computed. The same tests were repeated using independent f6:h and $\mathrm{f} 6: \mathrm{n}$ tallies. The combination of both tallies yielded results simialt to the $+\mathrm{f} 6$ tally. The individual proton tally correctly captures the physics of interest[62].

A series of more detailed simulations was developed in order to verify what MCNPX was doing while tracking the neutrons and the protons inside the scintillator. 

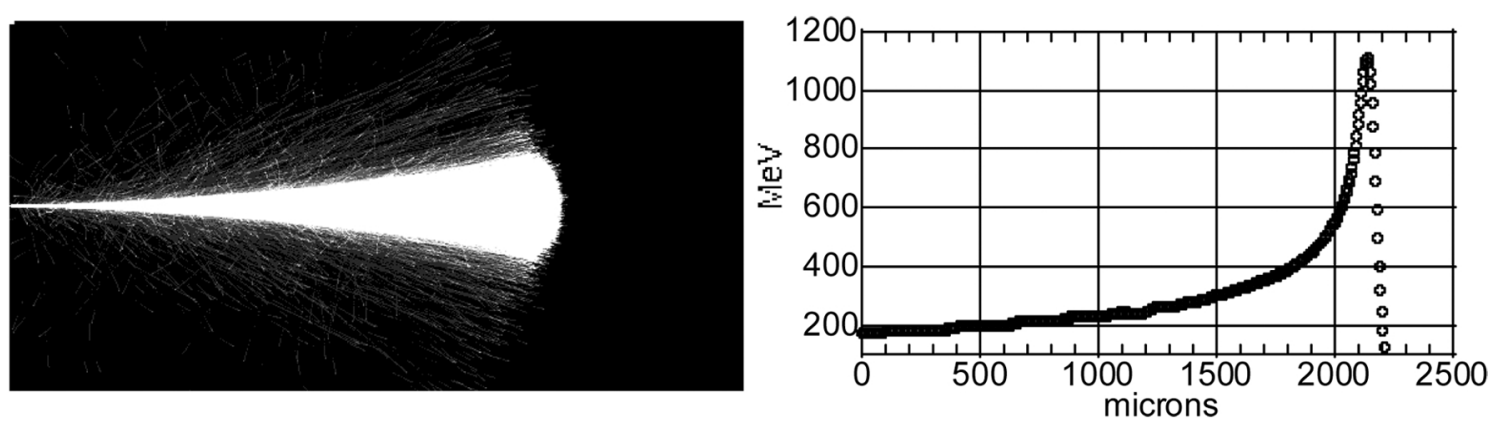

Figure 4.4: Simulation of a $14 \mathrm{MeV}$ proton beam impinging on a block of EJ232 scintillator. The image shows some of the ionization tracks caused by the protons. The plot shows the average value of energy deposition as measured in 5 micron cubic elements.

In these simulations a EJ232 scintillator block $\left(1.5 \times 1.5 \times 3 \mathrm{~mm}^{3}\right)$ is divided into 5 micron cubic cells and either a proton or neutron beam uniformly impinges on face on the central pixel. Figure 4.4 shows the side view of the scintillator when excited by a $14 \mathrm{MeV}$ proton beam. The value of each pixel represents the total energy deposited by protons in $\mathrm{MeV}$. The plot shows the average energy deposition in the vertical direction along the side face, this plot is easily recognizable as the Bragg curve for the protons, with a range of around $2.2 \mathrm{~mm}$. This curve is very similar to the one obtained using SRIM $^{2}$ and matches the range published in such references as Janni[63] and Andersend and Ziegler[64].

The actual energy deposition distribution in the scintillator is the combination of the neutron elastic scattering interactions and the range of the protons. Figure 4.5 shows the proton energy deposition for a $14 \mathrm{MeV}$ neutron beam. In this case no

\footnotetext{
${ }^{2}$ SRIM (Stopping and Ranges of Ions in Matter) is a public domain software package developed by James Ziegler. www.srim.org
} 

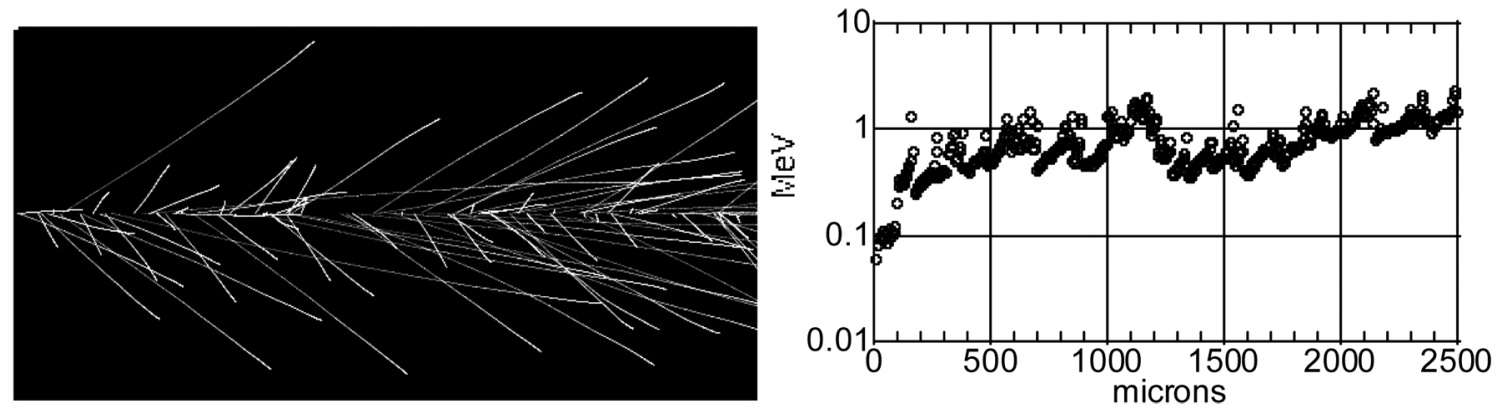

Figure 4.5: Simulation of a $14 \mathrm{MeV}$ neutron beam impinging on a block of EJ232 scintillator. The image shows some of the ionization tracks caused by the recoil protons. Notice that the energy deposition plot does not show the familiar Bragg peak anymore.

Bragg peak is visible and fewer $\mathrm{MeV} /$ pixel are recorded due to the low interaction probability of the neutrons in this thin scintillator.

The pixelated detector included in the End-to-End model is composed of 250micron square fibers $4.6 \mathrm{~cm}$ long. Illumination of the central fiber with $14 \mathrm{MeV}$ neutrons yields the distribution shown in figure 4.6. Most of the energy is deposited in the central pixel, which represents the fiber where the original neutron interaction would take place.

These calculations of the detector response and blur are conservative since they do not include corrections for the actual proton stopping power. According to Birk's formula

$$
\frac{\mathrm{d} L}{\mathrm{~d} x}=\frac{S \frac{\mathrm{d} E}{\mathrm{~d} x}}{1+k B \frac{\mathrm{d} E}{\mathrm{~d} x}}
$$

the light emission per unit length $(\mathrm{d} L / \mathrm{d} x)$ is proportional to the charged particle energy loss $(\mathrm{d} E / \mathrm{d} x)$, but a correction is introduced by the factor $k B$ in the denominator 

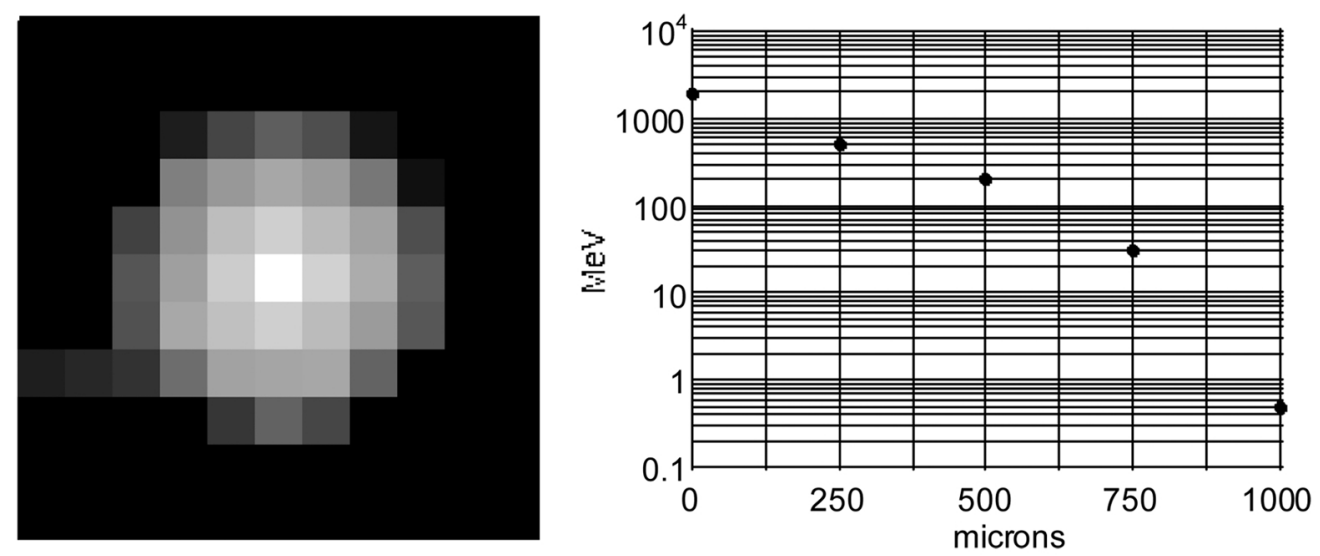

Figure 4.6: Proton energy deposition of a $14 \mathrm{MeV}$ neutron beam uniformly illuminating the central fiber of a simulated detector array. The image shows the front face view in a logarithmic grayscale. The plot shows the radial average of the enegy deposition

due to the self-quenching induced by high density ionization at the end of the tracks. This means that a proton emits less light around the Bragg peak due to internal quenching, but the MCNPX model shown here gives the same weight to all energy deposition, and in this way emphasizes the end of the ionization track. In reality the scintillator blur might be smaller. Lower energy neutrons will generate a smaller blur due to shorter ranges.

Based on these simulations it was decided that only the proton energy deposition will be tracked inside the scintillator using the f6:h tally, and that the neutron energy cut-off described in the previous section can be used without changing the scintillator response. The protons do not have an energy cut-off and are tracked until absorbed or when they leave the detector array. The blur introduced by the pixelated detector is integral part of the final images, no mathematical convolution is necessary. 


\subsubsection{Sample Input Deck}

An input deck corresponding to a single on-axis 5-micron square pinhole geometry,

a monoenergetic point source and a pixelated scintilaltor array is shown bellow. Lines starting with 'c' and a number are non-executable comments; they refer to detailed explanations given after the deck.

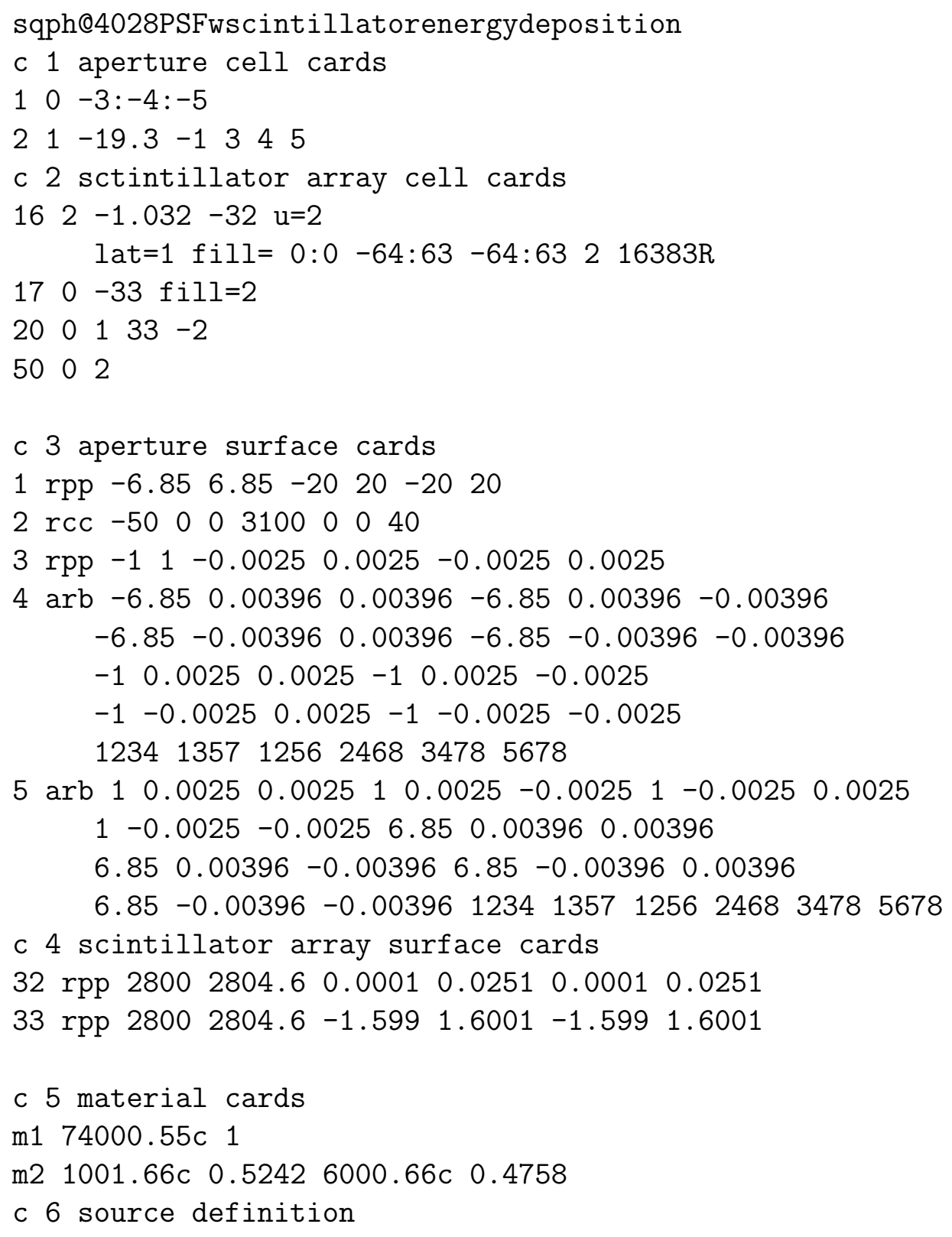




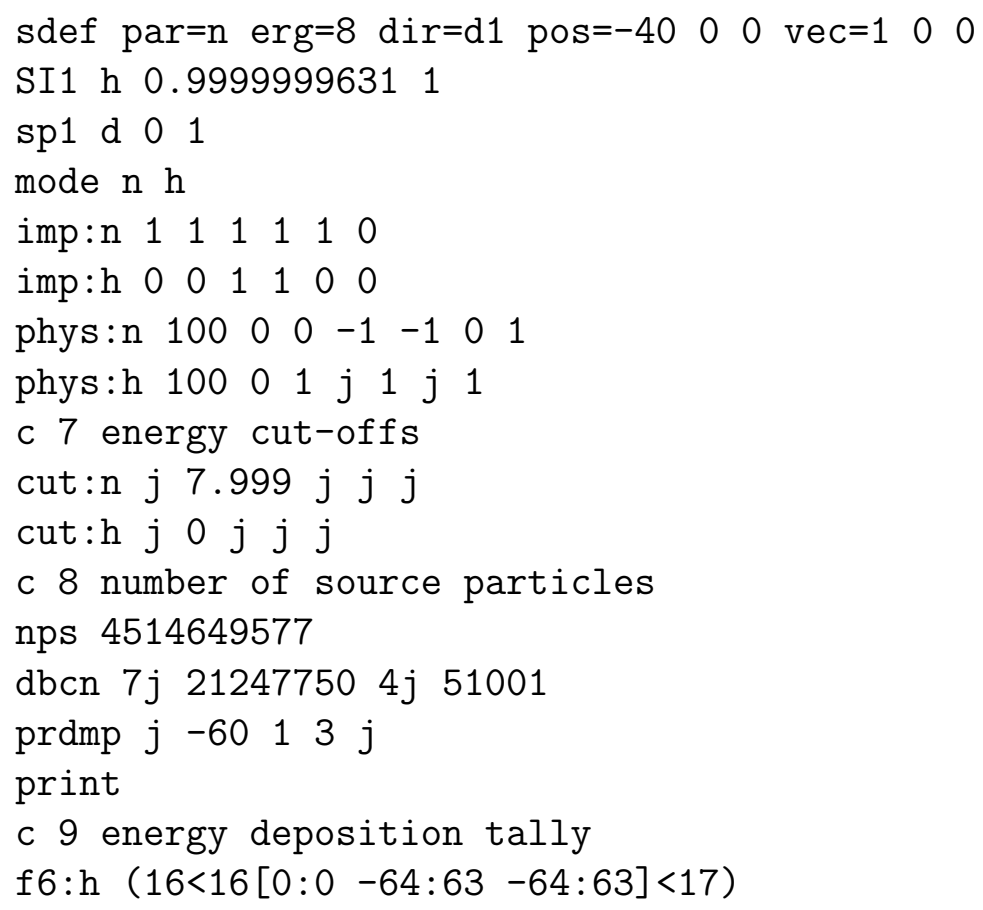

1. The square pinhole aperture is the intersection of three hollow cells: two truncated pyramids and a rectangular prism. They are inside a rectangular box.

2. The scintillator array is modeled using the 'fill' and 'lattice' commands. This simplifies the input deck and speeds up the calculation. The detector array is made of $128 \times 128250$-micron square fibers $4.6 \mathrm{~cm}$ long, but this syntax requires the exact description of just one fiber, which is in turn repeated 16383 times.

3. The center of the aperture is located at the origin $(0,0,0)$ as shown in figure 2.12 .

4. The front face of the detector array is located $28 \mathrm{~m}$ to the right of the aperture. The actual dimensions of the detector are $3.2 \times 3.2 \times 4.6 \mathrm{~cm}^{3}$. Although in 
reality the detector will use step-index fibers, the model assumes homogeneous composition across the square cross section.

5. The aperture is made of natural tungsten $(Z=74)$. The scintillator composition is that of EJ232 given as atomic percent of hydrogen $(Z=1 A=1)$ and natural carbon $(Z=6)$. No trace dopants are included.

6. The source is placed $40 \mathrm{~cm}$ to the left of the aperture. In this case it is an $8 \mathrm{MeV}$ neutron point source. The particles are emitted in a narrow cone oriented along the $\mathrm{X}$ axis and with angles given by the uniform sampling of the directinal cosine between 1 and 0.9999999631. MCNPX always assumes azimuthal symmetry. The source cone angle is such that only part of the detector is illuminated; this saves CPU time while keeping the counting statistics correct, but requires the 'patching' of the raw image in post-processing. In the case of a macroscopic source distribution the 'pos $=x \quad y \quad z$ ' term is replaced by a double valued distribution, location, where the source elements are, and intensity, based on the normalized intensity of each energy bin.

7. Neutron energy cut-off is used to reduce CPU time (see section 4.3.1.1).

8. The number of source particles in the problem is equivalent to the actual source strength times the solid angle fraction of the detector. The particle weight factors are all equal to one. In this particular case the original source strength was $1 \times 10^{17}$. 
9. The actual output of the run is the proton energy deposition in MeV per fiber.

The syntax used here matches the fill-lattice cell structure.

\subsection{Point Spread Functions}

The response of a system to an ideal point source is called point spread function (PSF) or impulse response. The PSF is a unique characteristic of each signal processing system; in the case of an imaging system it contains information about intensity modulation and any aberrations introduced by the system's components.

The PSF of each aperture-scintillator array combination can be generated using an input deck like the one described in the previous section. Figure 4.7 shows the PSFs of the five apertures considered in this study for a detector placed $40 \mathrm{~m}$ away and a magnification of $100^{3}$. Note that due to their large size, the annular and the penumbral apertures require $1024 \times 1024$ pixels arrays but are shown in the same size as the others.

Each PSF captures the shape of the aperture; the image generated is basically a radiograph of the high-Z collimator, and it shows a flat background or pedestal due to the direct transmission neutron flux. In many imaging systems the PSF is a square array which is small compared to the recorded image; the PSFs for the NIS are as big as the recorded images in order to account for the pedestal, which does not depend

\footnotetext{
${ }^{3}$ The actual magnification for the triangular wedge is only 85.3 due to location of the equivalent leverage point at $46.81 \mathrm{~cm}$ from TCC instead of $40 \mathrm{~cm}$ like in the other apertures.
} 


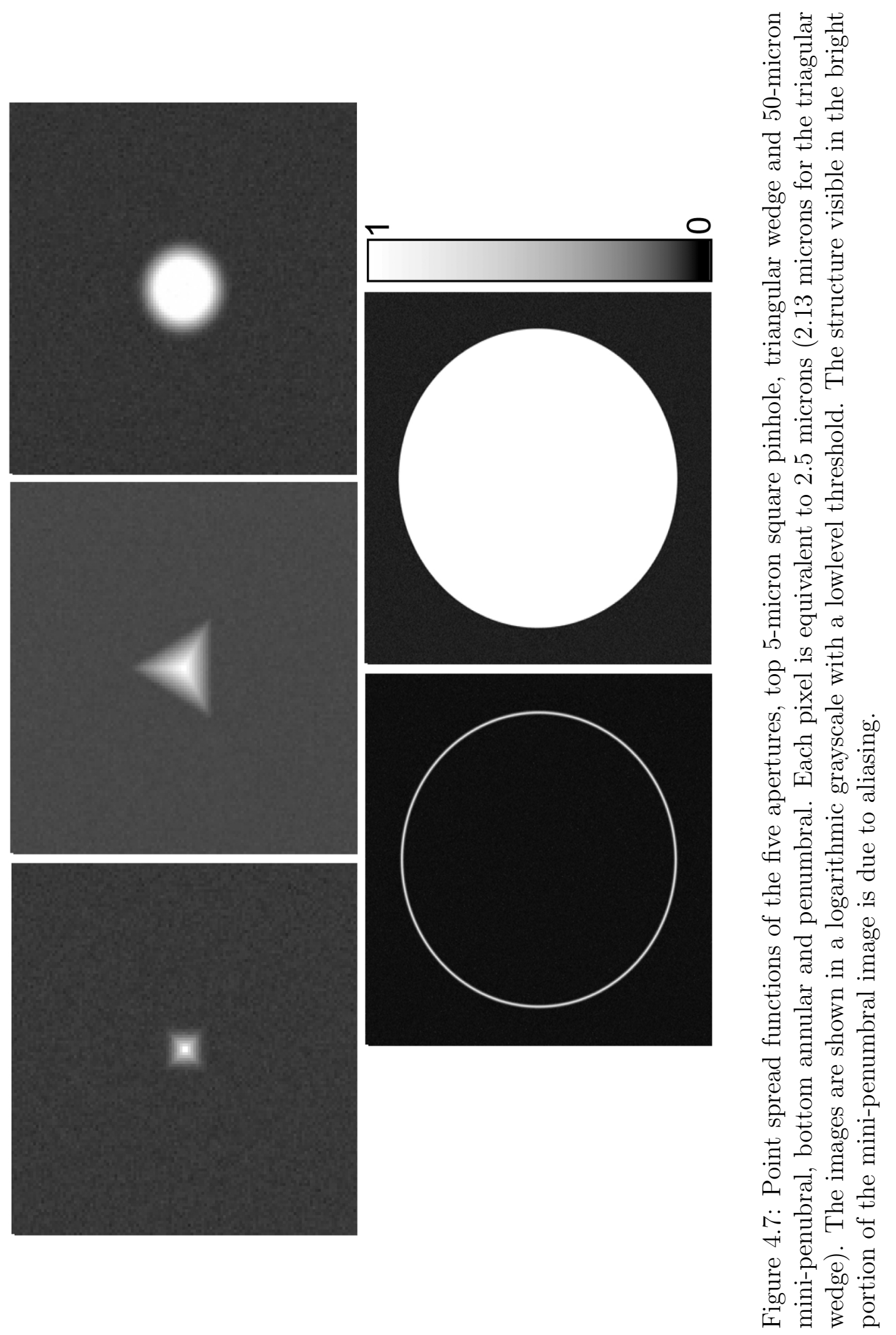




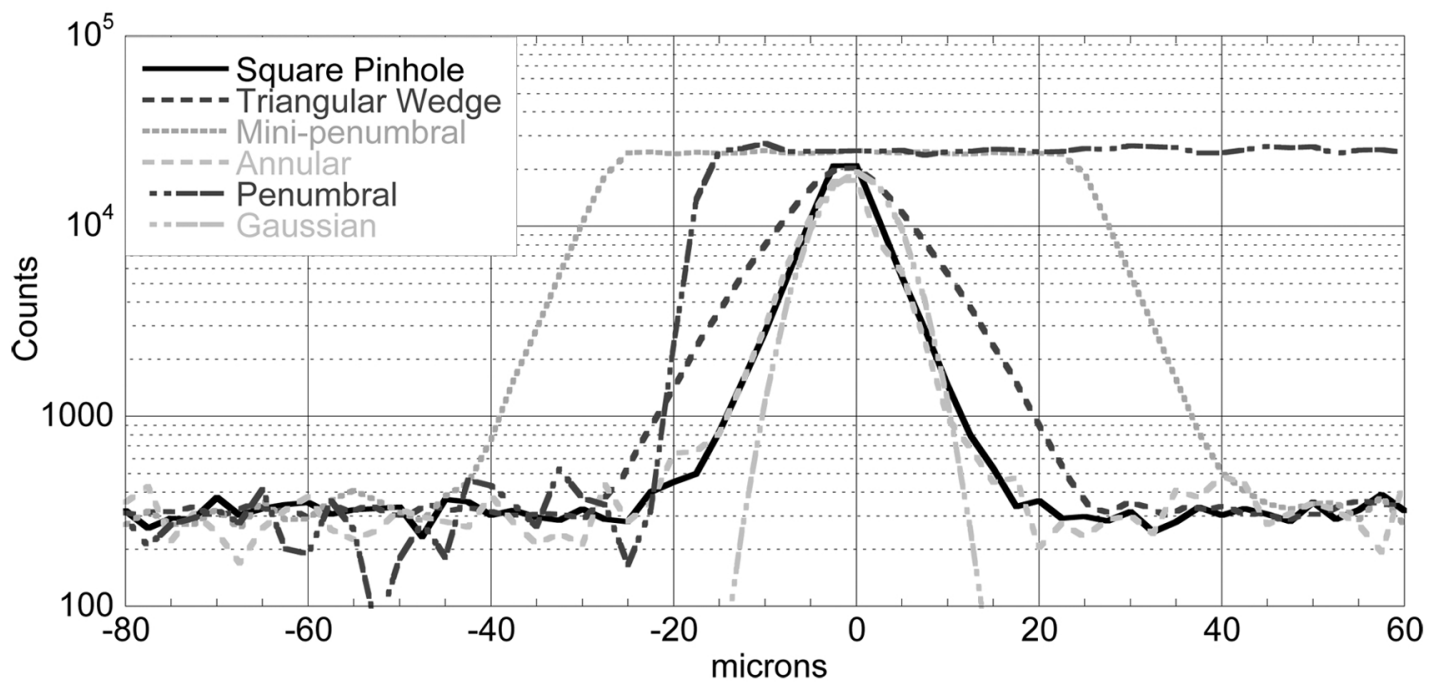

Figure 4.8: Vertical lineouts through the center of the PSF of the small apertures and a portion of the top of the annular and penumbral apertures. A 10 micron FWHM Gaussian has been included for comparison purposes.

on the source distribution and aperture profile shape, but on the source strength and aperture thickness. The benefit of this approach will be evident in chapter five, Image Processing. The plot in figure 4.8 shows vertical lineouts though the center of the pinholes and the top edge of the annular and penumbral apertures.

It has been common practice among target designers and physicists working in neutron imaging, to assume the system has a Gaussian shaped PSF, which has the advantage of having a simple mathematical form. However the lineouts show that Gaussian PSFs are not accurate models for real neutron apertures, where the PSFs are highly peaked and have sharp features. The apparent PSF width (FWHM) can be directly obtained from this plot, the results, including the Gaussian, are contained in table 4.1. 


\begin{tabular}{lcc}
\hline \hline Aperture & FWHM (microns) & $\phi_{\text {eff }}$ (microns) \\
\hline Square & 7.5 & \\
Triangular & 14.0 & 12.7 \\
Mini-penumbral & 56.0 & 27.2 \\
Annular & 8.0 & 59.0 \\
Penumbral & 2060 & 250.3 \\
Gaussian & 10.0 & NA \\
\hline \hline
\end{tabular}

Table 4.1: Apparent width and equivalent diameter for the different apertures based on their simulated PSFs.

A useful way to compare the PSF of these apertures side by side is to compute their equivalent diameter, i.e., the diameter of an opaque circular pinhole that allows the same neutron flux as the actual thick non-opaque version. The equivalent diameter is calculated based on the actual solid angle fraction subtended by the aperture $\Delta \Omega_{\mathrm{eff}}$, which can be written in terms of the number of non-background counts $N_{\mathrm{nb}}$ in the image and the number of source neutrons $N_{\text {source }}$ as

$$
\Delta \Omega_{\mathrm{eff}}=\frac{N_{\mathrm{nb}}}{N_{\mathrm{source}}}=\frac{A}{4 \pi x^{2}}
$$

where $x$ is the distance from the source to the leverage point of the aperture, which is $40 \mathrm{~cm}$, except for the triangular wedge which is located at $46.81 \mathrm{~cm} . N_{\text {source }}$ was kept consntat at $10^{16}$ neutrons. $A$ is the effective aperture area, which can be written as

$$
A=\pi \frac{\phi_{\mathrm{eff}^{2}}}{4}
$$

substituting 4.2 into 4.1 and solving for $\phi_{\text {eff }}$

$$
\phi_{\mathrm{eff}}=4 x\left(\frac{N_{\mathrm{nb}}}{N_{\text {source }}}\right)^{1 / 2}
$$


the effective diameter results appear in table 4.1. The annular aperture can also be considered as a slit wrapped around to form a circle. Using the same procedure described above and assuming an inner diameter of $2 \mathrm{~mm}$, the equivalent slit width is 7.8 microns

\subsection{Image Formation with Scintillator Decay}

The next step in the End-to-End model is to generate a raw recorded image; each individual energy deposition array needs to be corrected for the scintillator decay, depending on its time of arrival, and then combined to form the desired image. From the image formation point of view, the quantity of interest is the scintillation light fraction that is emitted and captured by the imaging system during the primary or downscattered recording windows. These fractions can be calculated using the light emission decay profile assuming that all the light generated by one interaction is emitted between -2 and 800 ns with respect to maximum emission.

Figure 4.9 shows the decay profile for EJ232, the integrated light emission and the two windows for a $40 \mathrm{~m}$ LOS. Table 4.2 contains the light emission fractions for detectors placed at 28 and $40 \mathrm{~m}$, for each energy bin and for the two gating windows. 


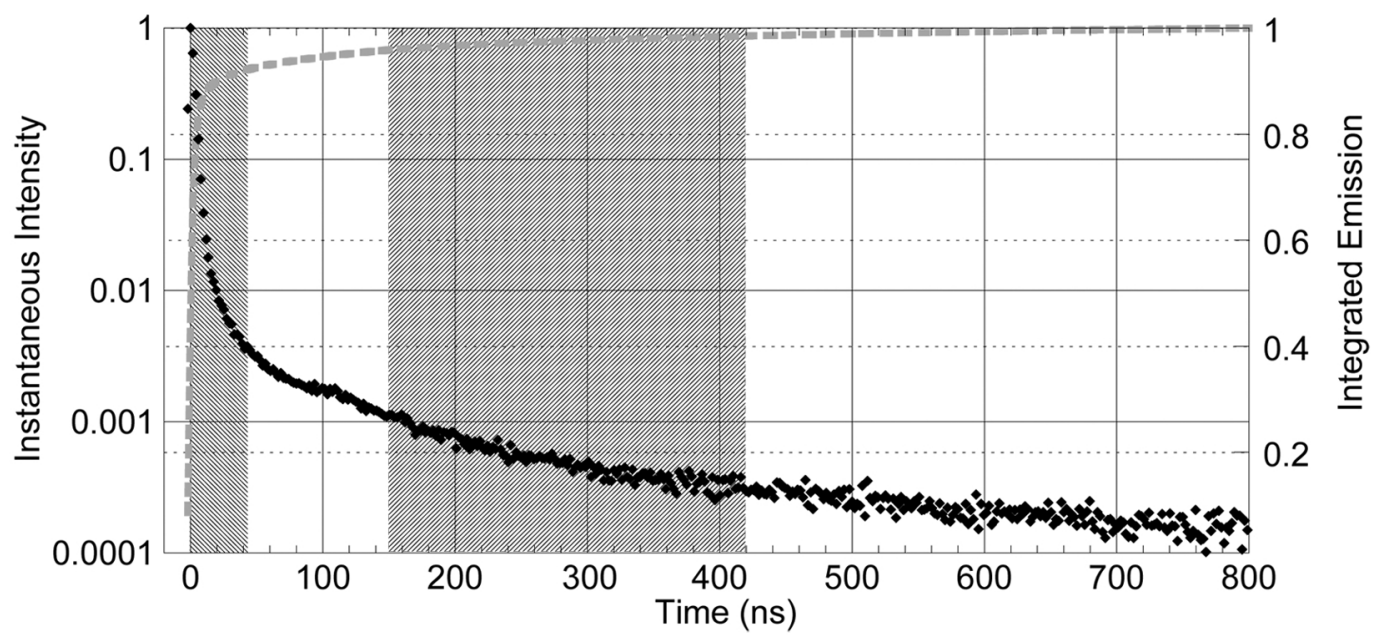

Figure 4.9: Light emission decay profile (left) and integrated emission (right) for EJ232 excited by $14 \mathrm{MeV}$ neutrons. The two shaded areas represent the primary and downscattered gating windows for a primary neutron and a $40 \mathrm{~m}$ LOS.

\begin{tabular}{ccccc}
\hline \hline & & & & \\
$E_{n}$ & $\mathbf{2 8} \mathbf{~ m}$ & $\mathbf{2 8} \mathbf{~ m}$ & $\mathbf{4 0} \mathbf{~ m}$ & $\mathbf{4 0} \mathbf{~ m}$ \\
$(\mathbf{M e V})$ & Primary & Downscattered & Primary & Downscattered \\
\hline 14.5 & 0.91117 & 0.02843 & 0.91769 & 0.02686 \\
13.5 & 0.86991 & 0.03303 & 0.88205 & 0.03141 \\
12.5 & - & 0.04080 & - & 0.03887 \\
11.5 & - & 0.04887 & - & 0.04979 \\
10.5 & - & 0.07874 & - & 0.07839 \\
9.5 & - & 0.96255 & - & 0.97055 \\
8.5 & - & 0.95498 & - & 0.96361 \\
7.5 & - & 0.94169 & - & 0.95069 \\
6.5 & - & 0.91365 & - & 0.92019 \\
\hline \hline
\end{tabular}

Table 4.2: EJ232 light emission fractions for each energy bin during the primary and downscattered gating windows and at 28 and $40 \mathrm{~m}$. 


\subsubsection{Zero Degradation Case}

Reference-type primary and downscattered images can be obtained by direct combination of the $P_{6}$ neutron source distributions and the light emission fractions. These images, which do not show any kind of blur, have perfect statistics and preserve a 2.5 micron pixelation; can be used later to compare the imaging performance of the LOS configurations.

Figure 4.10 shows the two simulated images for a $40 \mathrm{~m}$ LOS along with a plot of the horizontal lineouts through the center of each energy bin contribution to the 6-10 MeV image. The primary image appears very sharp and undistinguishable from the individual images shown at the bottom of figure 4.2. The downscattered image looks very different from the pure $6-10 \mathrm{MeV}$; it is contaminated by the afterglow of the primary neutrons. Even though the light fraction of the 14.5-13.5 MeV neutrons present in the downscattered image is only $2.69 \%$ and $3.14 \%$ respectively, due to their high yield they dominate the center of the 6-10 MeV image. The 12.5 to $10.5 \mathrm{MeV}$ contributions are small due to the big drop in the neutron spectrum after $13 \mathrm{MeV}$.

The downscattered image has limited usefulness, and improvements from modifications to the scintillator and the length of the LOS are impractical. An alternative based in post-processing consists of recording both images independently, and then carefully subtracting the fraction of the primary image (14.5-13.5 MeV) present in the downscattered one. Figure 4.11 compares the downscattered image after such processing with the original pure 6-10 MeV reference. The two images are very similar. 


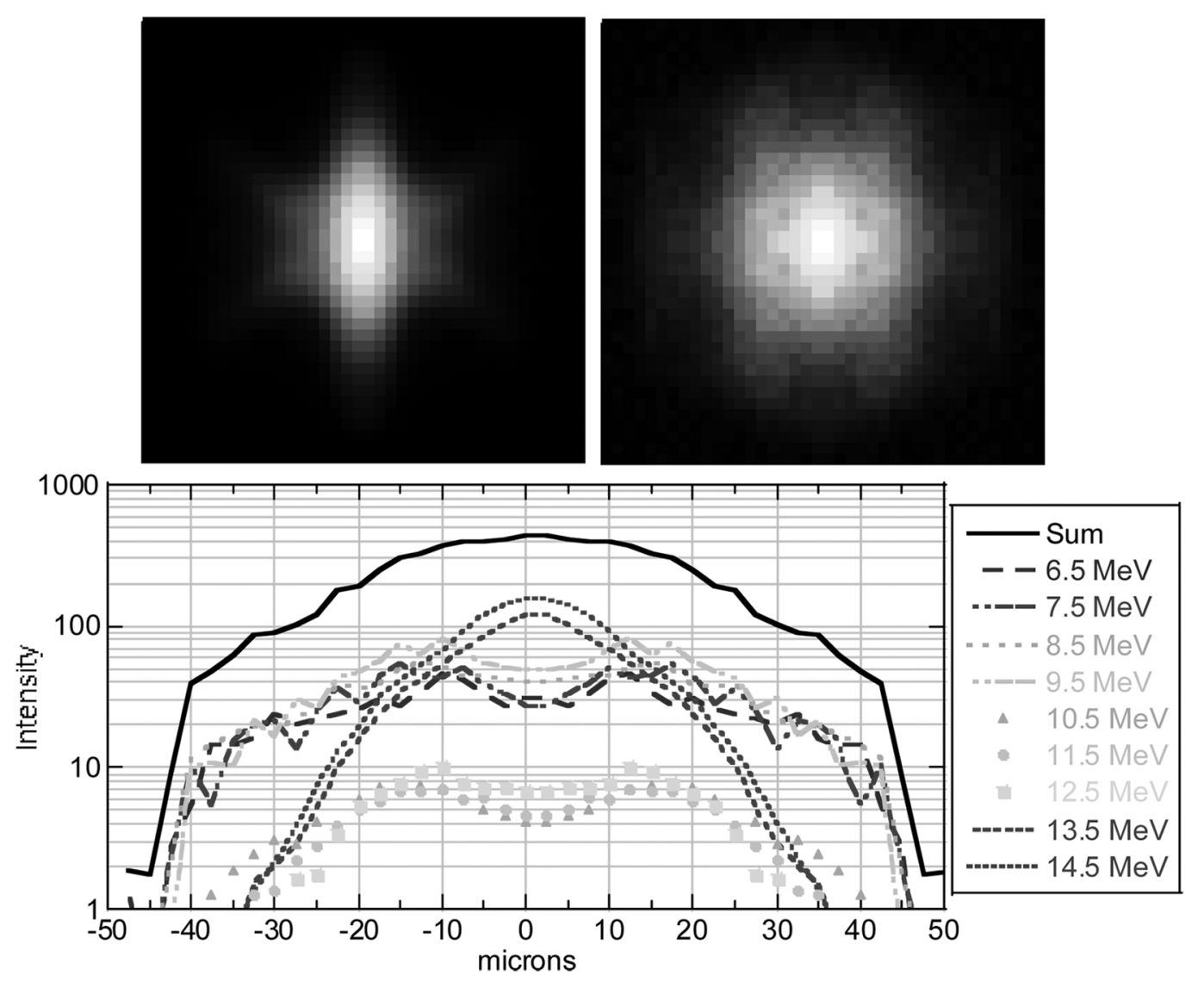

Figure 4.10: Ideal primary (left) and downscattered (right) images at $40 \mathrm{~m}$. The plot shows the contribution of each energy bin to the final downscattered image.

\subsubsection{Image Subtraction Algorithm}

Figure 4.12 shows the algorithm used to generate the raw primary and downscattered images and then subtract the afterglow contamination from the latter.

MCNPX generates an energy deposition array $(\mathrm{MeV} /$ pixel) of each of the nine neutron source distributions. The arrays are then multiplied by the corresponding 

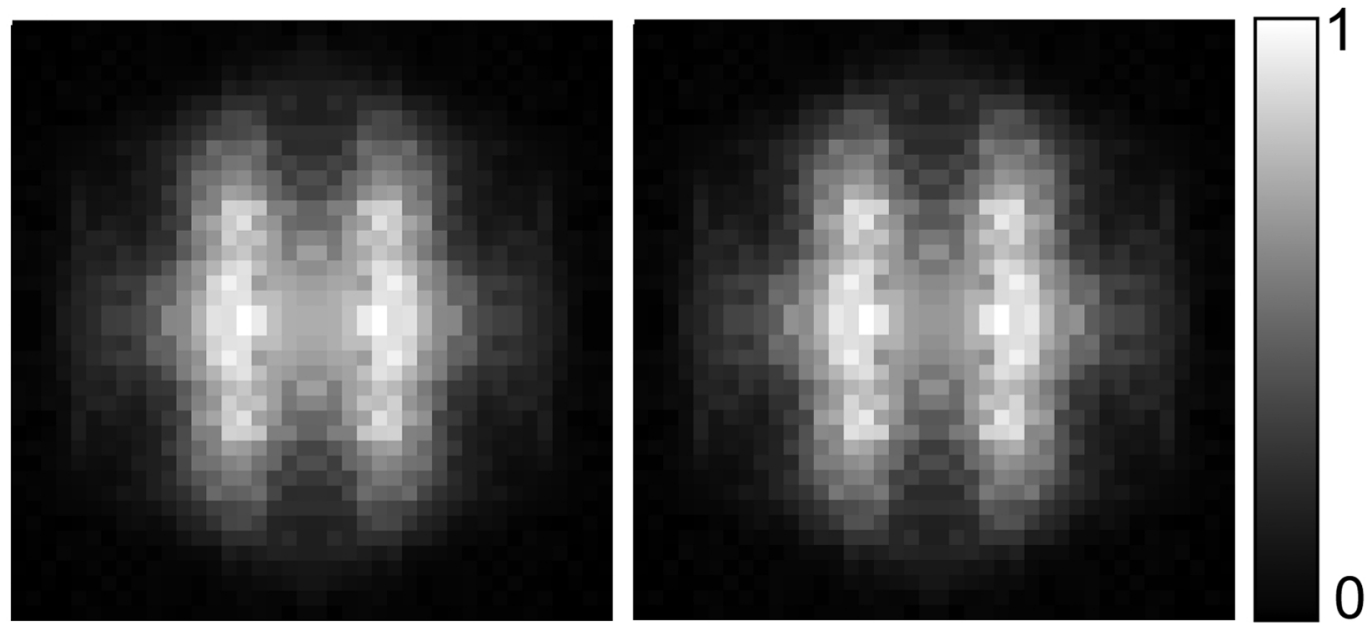

Figure 4.11: Downscattered image afer subtraction of the primary afterglow (left) and pure 6-10 MeV image (right). Both iamges look very similar, the only difference is a slightly brighter central region for the subtracted.

scintillator light emission fraction, the average number of photons generated per $\mathrm{MeV}$ (4285 based on 30000 photons for a $7.5 \mathrm{MeV}$ proton), a light collection fraction (0.001) representing a fast optical system and the photocathode efficiency (0.2) of a fictitious MCP. These new arrays are given in photo-electrons per pixel. The raw primary image is generated by adding the 13.5 and $14.5 \mathrm{MeV}$ arrays pixel-by-pixel. The raw downscattered image or 'SUM' is generated in three steps, first the raw primary is multiplied by the light emission fraction present in the 6-10 $\mathrm{MeV}$ gate (afterglow), then this decayed array and the individual ones (6.5 to $12.5 \mathrm{MeV})$ are sampled with a Poisson distribution, which represents the random photoelectron emission, and finally these new arrays (6.5 to $14.5 \mathrm{MeV}$ ) are summed pixel-by-pixel. The 'DIFFERENCE' image is obtained by subtracting the decayed non-Poisson primary from the SUM. 


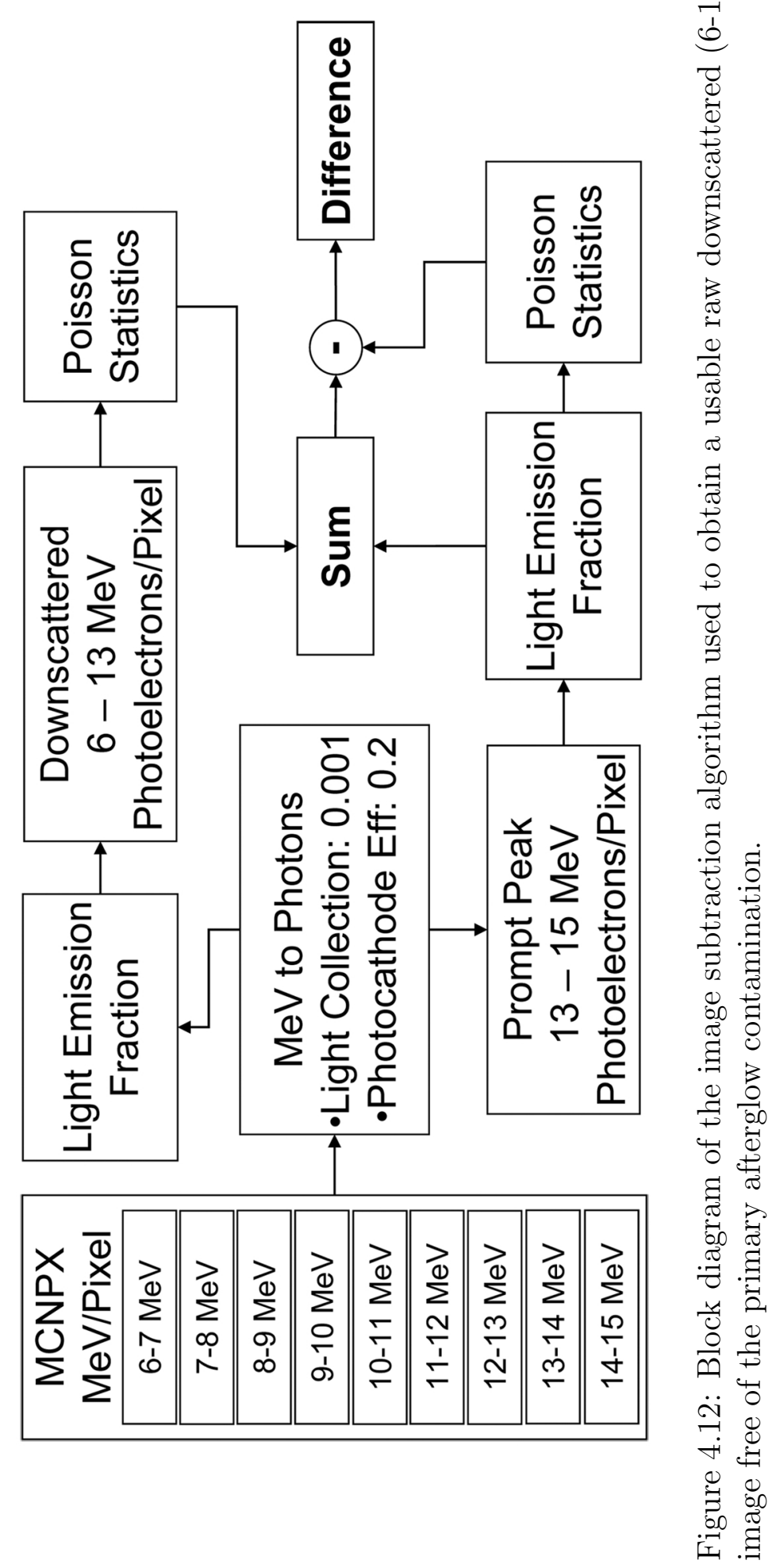




\subsubsection{Raw Images}

Simulations of a single square pinhole, triangular wedge or mini-penumbral aperture show that the neutron throughput is very low to form useful downscattered images, while the primary ones are marginally good in some cases. The neutron flux can be increased by using an array of apertures pointed toward TCC. The individual images can then be added in post-processing to obtain a single image of increased quality. Although the complete pinhole array can be modeled in MCNPX, the baseline simulations were performed using a single on-axis aperture with a number of source particles equal to the original intensity times the number of apertures in the array. For the $40 \mathrm{~m} \operatorname{LOS}$ a $10 \times 10$ array was used, while for the $28 \mathrm{~m}$ case it was a $7 \times 7$ array, but no triangular wedge was included. In both cases the annular and penumbral apertures remained the same.

The simulations were performed on a serial PC-based system and the THUNDER supercomputer at LLNL, which is a Linux-based parallel system. The nine source set plus PSF took on average three days to complete.

Figures 4.13 and 4.14 show the primary, sum and difference images for the different apertures at 40 and $28 \mathrm{~m}$ respectively. Each image shows the complete array and is normalized to its maximum (white). 

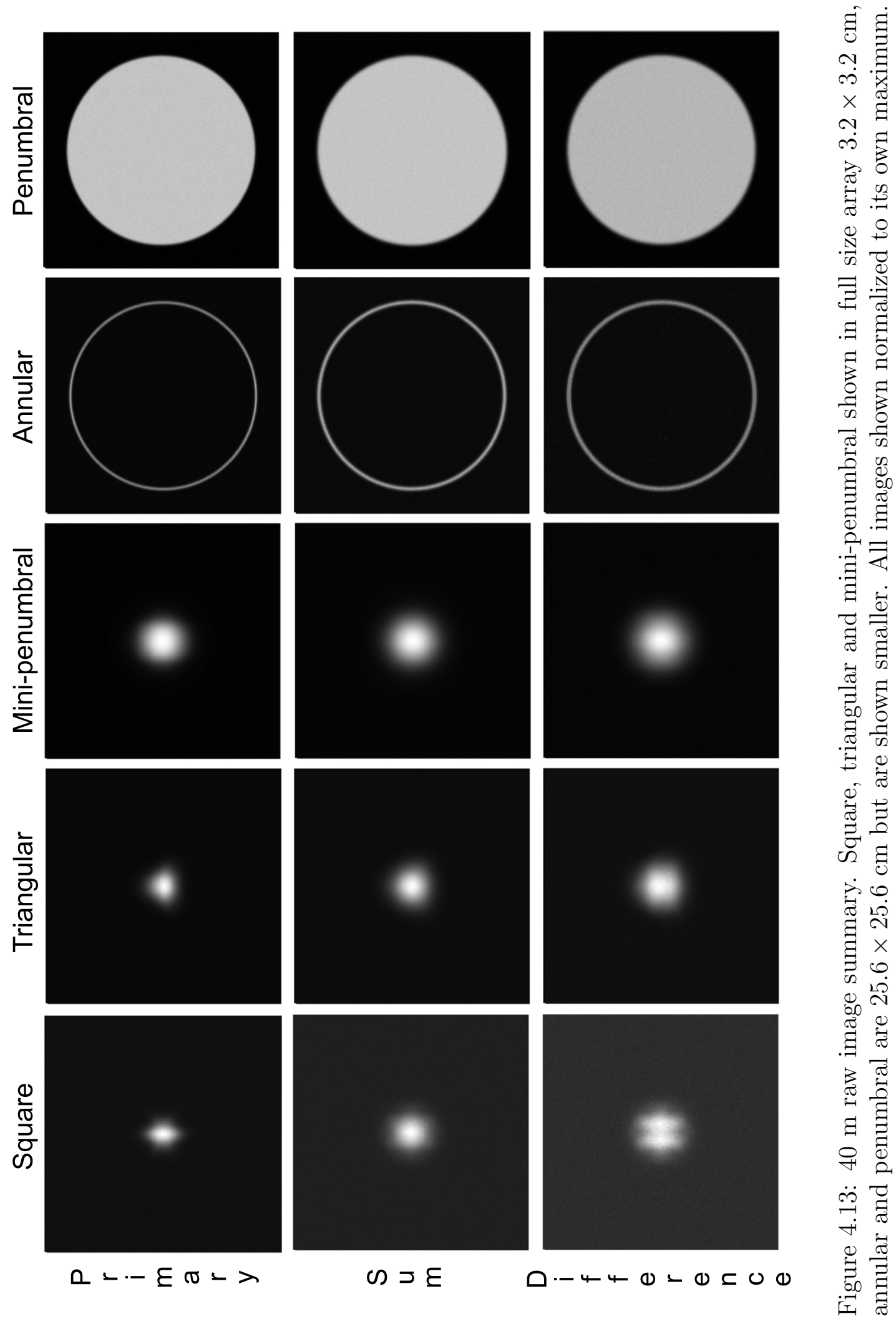

$\cap--\varepsilon \sigma->$

$\circlearrowleft \sqsupset \varepsilon$

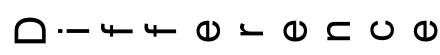



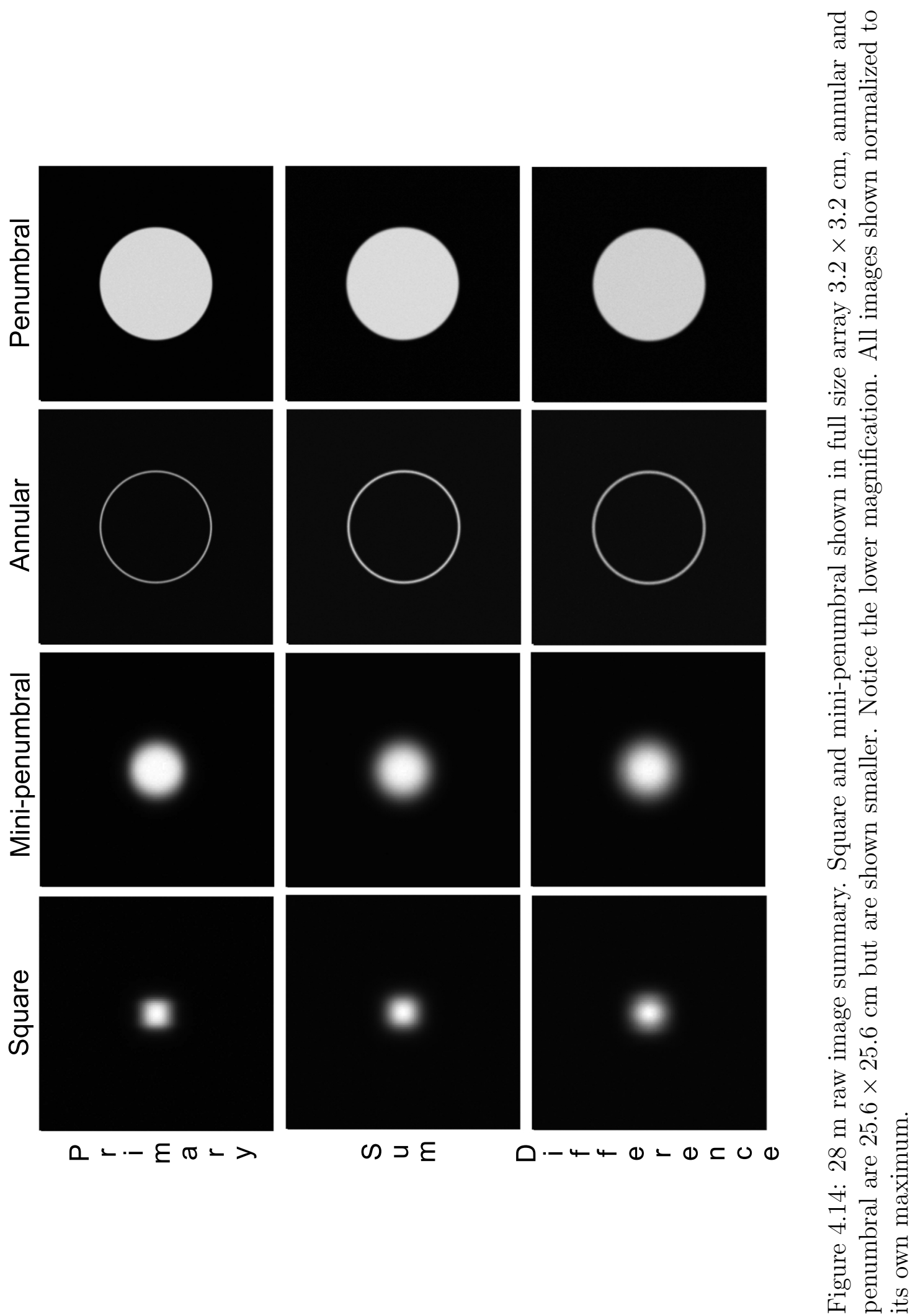


\section{Chapter 5}

\section{Digital Image Processing}

Most of the raw primary and downscattered difference images generated using the MCNPX-based model are highly blurred, making them unfit for neutron imaging purposes; only the square pinhole produces an image that resembles the original source. The penumbral and annular apertures are extreme cases in which no trace of the original source can be found since it has been coded into a large bright spot or a ring respectively. The only physical solution for the pinhole apertures would be to make them very long and narrow in order to reduce their effective diameters, but this is problematic because of manufacturing difficulties and the neutron throughput maks the images more difficult to record.

Fortunately there is the possibility of mathematical processing of the raw image in order to recover an improved final source. This chapter presents the basic mathematical treatment of image formation and processing, including the linear system 
approximation and the concepts of convolution and frequency domain filtering. These tools are used to deconvolve the raw images using regularized inverse filtering and an autocorrelation method, to compare the imaging qualities of the apertures and to find the optimal filtering required to generate a useful final image.

\subsection{Mathematical Treatment}

In many ways neutron imaging is similar to visible light imaging (photography) or medical imaging (radiography or Positron Emission Tomography). Imaging, in general, can be modeled as a mapping function that takes a multidimensional (space and/or time) source distribution $\mathbf{m}_{1}^{\text {in }}$ and transforms it into a two dimensional array of pixel values (counts, intensity, etc) $\mathbf{m}_{1}^{\text {out }}$. In an ideal imaging system each source element would be imaged onto a single pixel, or, depending on the magnification and oversampling of the CCD camera, onto a set of pixels. In reality the value of an output pixel is influenced by neighboring source elements; this so-called nonlocal behavior was already apparent in the simulated images of chapter four.

Some simplifications are necessary in order to make the system modeling practical; the most important of these is that the imaging system is linear, i.e., given $\mathbf{m}_{1}^{\text {in }} \Rightarrow \mathbf{m}_{1}^{\text {out }}$ and $\mathbf{m}_{2}^{\text {in }} \Rightarrow \mathbf{m}_{2}^{\text {out }}$, then $\alpha \mathbf{m}_{1}^{\text {in }}+\beta \mathbf{m}_{2}^{\text {in }} \Rightarrow \alpha \mathbf{m}_{1}^{\text {out }}+\beta \mathbf{m}_{2}^{\text {out }}, \alpha$ and $\beta$ are a real numbers. Using the linear superposition principle, the nonlocal behavior can be written in one 
dimension as

$$
\mathbf{m}^{\text {out }}(u)=\int_{-\infty}^{\infty} p\left(u ; u^{\prime}\right) \mathbf{m}^{\text {in }}\left(u^{\prime}\right) \mathrm{d} u^{\prime}
$$

the term $p\left(u ; u^{\prime}\right)$ represents the functional relation between source elements and image pixels. Further insight into the nature of $p\left(u ; u^{\prime}\right)$ can be gained using Dirac's $\delta$-function as an input distribution

$$
\mathbf{m}^{\text {out }}(u)=\int_{-\infty}^{\infty} p\left(u ; u^{\prime}\right) \delta\left(u^{\prime}-u_{o}\right) \mathrm{d} u^{\prime}
$$

using the properties of the $\delta$-function 5.2 becomes

$$
\mathbf{m}^{\text {out }}(u)=p\left(u ; u_{o}\right)
$$

which says that $p\left(u ; u_{o}\right)$ is the point spread function of the system, i.e., the response of the system measured at a pixel $u$ to a sharp impulse located at $u_{o}$. The PSFs illustrated in section 4.4 have $u_{o}=(0,0)$.

Another important simplification commonly used in the modeling of imaging systems is to consider them shift-invariant, this is, a shift in the location of a point source will result in a shift in the location of the image, but its shape and intensity will remain the same. This kind of system is also known as isoplanatic and can be represented by writing

$$
\mathbf{m}^{\text {out }}(u)=\int_{-\infty}^{\infty} p\left(u-u^{\prime}\right) \mathbf{m}^{\text {in }}\left(u^{\prime}\right) \mathrm{d} u^{\prime}
$$

where now the PSF is in terms of the distance, or shift, between the two locations. Equation 5.4 is also the definition of the mathematical operation convolution[65], 
which can be compactly written as

$$
\mathbf{m}^{\text {out }}(u)=p(u) * \mathbf{m}^{\text {in }}(u)
$$

and working explicitly in two dimensions in rectangular coordinates

$$
\mathbf{m}^{\text {out }}(x, y)=p(x, y) * \mathbf{m}^{\text {in }}(x, y)=\int_{-\infty}^{\infty} \int_{-\infty}^{\infty} p\left(x-x^{\prime}, y-y^{\prime}\right) \mathbf{m}^{\text {in }}\left(x^{\prime}, y^{\prime}\right) \mathrm{d} x^{\prime} \mathrm{d} y^{\prime}
$$

One last simplification can be introduced by studying the response of a one dimensional system to a single complex exponential input of the form

$$
\mathbf{m}^{\text {in }}(u)=\exp (2 \pi i \nu u)
$$

where $i=\sqrt{-1}$ and $\nu$ is a spatial frequency. The output distribution can be written as

$$
\mathbf{m}^{\text {out }}(u)=\int_{-\infty}^{\infty} p\left(u-u^{\prime}\right) \exp \left(2 \pi i \nu u^{\prime}\right) \mathrm{d} u^{\prime}
$$

changing variables $u^{\prime \prime}=u-u^{\prime}$ and rearranging

$$
\mathbf{m}^{\text {out }}(u)=\exp (2 \pi i \nu u) \int_{-\infty}^{\infty} p\left(u^{\prime \prime}\right) \exp \left(-2 \pi i \nu u^{\prime \prime}\right) \mathrm{d} u^{\prime \prime}
$$

the integral in 5.9 is the Fourier transform[66] of $p\left(u^{\prime \prime}\right)$ which is defined as

$$
P(\nu)=F\{p(u)\}=\int_{-\infty}^{\infty} \exp (-2 \pi i \nu u) p(u) \mathrm{d} u
$$

$P(\nu)$ is the system's transfer function or simply the transform of the PSF, with which the output distribution can be written as

$$
\mathbf{m}^{\text {out }}(u)=P(\nu) \exp (2 \pi i \nu u)
$$


The general case of an input distribution of complex exponentials can be written, using the definition of inverse Fourier transform

$$
m(u)=F^{-1}\{M(u)\}=\int_{-\infty}^{\infty} \exp (2 \pi i \nu u) M(\nu) \mathrm{d} \nu
$$

and the superposition principle, as

$$
\mathbf{m}^{\text {out }}(u)=\int_{-\infty}^{\infty} M^{\text {in }}(\nu) P(\nu) \exp (2 \pi i \nu u) \mathrm{d} \nu
$$

The importance of this expression is not obvious, but by noticing that the right-hand side has the form of an inverse Fourier transform and by using the property

$$
F^{-1}\{F\{m(u)\}\}=m(u)
$$

5.13 can be written as

$$
M^{\text {out }}(\nu)=M^{\text {in }}(\nu) P(\nu)
$$

Thus, the Fourier transform of the image distribution is equal to the product of the Fourier transforms of PSF and the source distribution.

According to 5.5 the image distribution, in real Cartesian space, is equal to the convolution of the PSF and the source, applying the Fourier transform to both sides of 5.5 and using 5.4

$$
M^{\text {out }}(\nu)=F\left\{p(u) * \mathbf{m}^{\mathrm{in}}(u)\right\}=\int_{-\infty}^{\infty} \int_{-\infty}^{\infty} p\left(u-u^{\prime}\right) \mathbf{m}^{\mathrm{in}}\left(u^{\prime}\right) \exp (-2 \pi i \nu u) p(u) \mathrm{d} u \mathrm{~d} u^{\prime}
$$

changing variables $u^{\prime \prime}=u-u^{\prime}$ and rearranging

$$
F\left\{p(u) * \mathbf{m}^{\mathrm{in}}(u)\right\}=\int_{-\infty}^{\infty} p\left(u^{\prime \prime}\right) \exp \left(-2 \pi i \nu u^{\prime \prime}\right) \mathrm{d} u^{\prime \prime} \int_{-\infty}^{\infty} \mathbf{m}^{\mathrm{in}}\left(u^{\prime}\right) \exp \left(-2 \pi i \nu u^{\prime}\right) \mathrm{d} u^{\prime}
$$


which is nothing more than

$$
F\left\{p(u) * \mathbf{m}^{\text {in }}(u)\right\}=P(\nu) M^{\text {in }}(\nu)
$$

The power of 5.15 and 5.18 is now evident: the convolution of two functions in real space is equivalent to the multiplication of their Fourier transforms, this saves time and computational resources.

In reality the source distributions and the output images are not continuous functions but discrete arrays of real numbers. The integrals used in the above mathematical expressions need to be replaced by summations in two dimensions and the Fourier transforms by the Discrete Fourier Transform (DFT) [66]

$$
M(\omega, \nu)=\frac{1}{a b} \sum_{j=0}^{a-1} \sum_{k=0}^{b-1} m(j, k) \exp \left(-2 \pi i\left(\frac{\omega j}{a}+\frac{\nu k}{b}\right)\right)
$$

where $\omega$ and $\nu$ are the spatial frequencies associated with $x$ and $y$ respectively, $a$ and $b$ are the horizontal and vertical size of the array ${ }^{1}$, and $j$ and $k$ give the pixel location inside the array according to

$$
j=\frac{x}{a} \quad k=\frac{y}{b}
$$

The DFT shares the mathematical properties of the continuous version and can be implemented in numerical calculations through multiple algorithms, the most popular being the Cooley-Tukey fast Fourier transform (FFT)[67].

A filter in frequency space is an independent function $R(\omega, \nu)$ that can be used to

\footnotetext{
${ }^{1}$ For this study all the arrays are square, so $a=b$.
} 
modify or modulate the output image, which is accomplished by simple multiplication

$$
M_{f}(\omega, \nu)=M(\omega, \nu) R(\omega, \nu)
$$

The frequency filter can have almost any shape, but the most common types are low-pass (smoothing) and high-pass (sharpening)[68][69], the 'low' and 'high' refer to the frequencies left unchanged.

A special type of filter is the inverse filter $R_{i}(\omega, \nu)$, which is an idealized filter that has the property of cancelling out the effects of previous filters or convolutions

$$
m_{2}(x, y)=m_{1}(x, y) * m_{0}(x, y) \Longrightarrow m_{2}(x, y) * m_{i}(x, y)=m_{0}(x, y)
$$

in frequency space

$$
\begin{aligned}
\left(M_{1}(\omega, \nu) M_{0}(\omega, \nu)\right) R_{i}(\omega, \nu) & =M_{0}(\omega, \nu) \\
R_{i}(\omega, \nu) & =\frac{1}{M_{1}(\omega, \nu)} \\
r_{i}(x, y) & =\int_{-\infty}^{\infty} \int_{-\infty}^{\infty} \frac{1}{M_{1}(\omega, \nu)} \exp (2 \pi i(\omega x+\nu y) \mathrm{d} \omega \mathrm{d} \nu
\end{aligned}
$$

$R_{i}(\omega, \nu)$ is idealized because it can not contain any zeros or very small values that would cause the integral to diverge; and most of the time there is no exact analytical form, just experimental or mathematical approximations. The importance of the inverse filter becomes apparent if the PSF is considered as another filter; it might be possible to find an inverse filter that cancels the effects of the PSF and allows the recovery of the original source.

At this point it is convenient to introduce a more precise notation. $o(x, y), \operatorname{im}(x, y)$ and $p s f(x, y)$ represent the object or source, the image and the system's PSF in real 
space. $O(\omega, \nu), \operatorname{Im}(\omega, \nu)$ and $P S F(\omega, \nu)$ are their respective Fourier transforms. The image can then be written as

$$
\begin{aligned}
i m(x, y) & =o(x, y) * \operatorname{psf}(x, y) \\
\operatorname{Im}(\omega, \nu) & =O(\omega, \nu) \operatorname{PSF}(\omega, \nu)
\end{aligned}
$$

and the recovered source or object as

$$
O(\omega, \nu)=\frac{\operatorname{Im}(\omega, \nu)}{\operatorname{PSF}(\omega, \nu)}
$$

\subsection{Implementation}

The image processing tasks were implemented with the commercially available software package MathCAD $11^{2}$ for PC. The advantages of MathCAD 11 include the ease and transparency of the implementation and the possibility of comparing and replicating the neutron imaging work conducted at CEA.

MathCAD 11 provides several FFT commands[70]; this study used exclusively cfft and icfft for the direct and inverse FFT. The input of cfft and the output of icfft are square arrays of real numbers that can be saved as tab-limited text files. The size of the input array is not limited to $2^{n}$ as in the original FFT algorithm, but for convenience $n=7$ and $n=10$ were used.

\footnotetext{
${ }^{2}$ http://www.ptc.com/appserver/mkt/products/home.jsp?k=3901 PTC Corporate Headquarters 140 Kendrick Street Needham, MA 02494 USA Phone: (781) 370-5000
} 


\subsubsection{Power Spectra and Modulation Transfer Function}

The output of cfft is in general a square array of complex numbers. The best way to visualize one of these arrays is to plot its power spectrum, which is given by

$$
P\{M(\omega, \nu)\}=|M(\omega, \nu)|^{2}=\operatorname{Re}^{2}(M(\omega, \nu))+\operatorname{Im}^{2}(M(\omega, \nu))
$$

The power spectrum is a $2 \mathrm{D}$ function of spatial frequencies $\omega$ and $\nu$, where the center pixel represents the DC component of the image, which corresponds to the average level in the space domain. The spatial frequencies increase with the radius and the power spectrum tends to have mirror symmetry.

Due to the way the FFT is computed, its output must be rearranged in order to display it in an understandable way. In this study the power spectra and intermediate complex arrays are reorganized by multiplying them by the square array

$$
t(x, y)=(-1)^{x+y}
$$

which shifts the origin from the upper left corner to the center ${ }^{3}$. Figure 5.1 shows the raw power spectrum of the five micron square pinhole PSF and the reorganized version. Figure 5.2 shows the power spectra of the PSFs at $40 \mathrm{~m}$. All the PSFs show a pronounced peak at $(\omega, \nu)=(0,0)$ which is due to the large number of pixels that form the direct transmission pedestal in the real space image; this is particularly evident in the penumbral and annular images, where very few pixels are actually related to the coded source.

\footnotetext{
${ }^{3}$ This is based on the translation properties of the DFT. See Gonzalez and Woods[69] for a detailed explanation.
} 

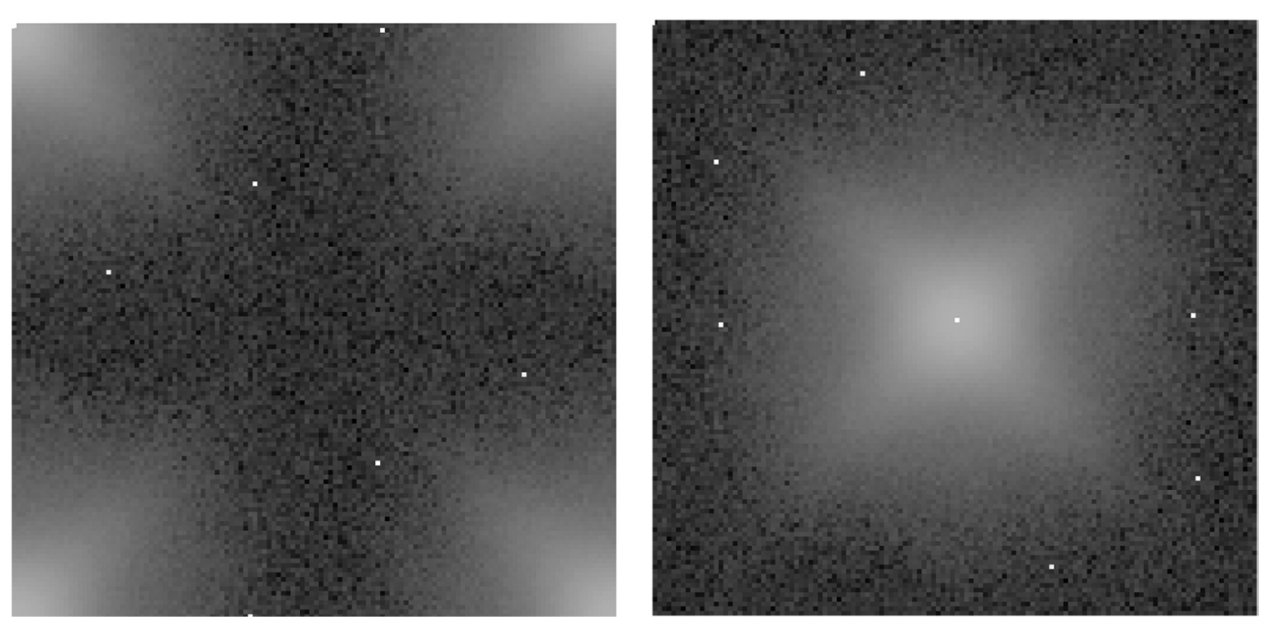

Figure 5.1: Power Spectrum of the 5-micron square pinhole PSF. Left: Raw array produced by the FFT Right: Reorganized array with DC component in the center $(64,64)$. Images are normalized to their maximum (white) and shown in a logarithmic grayscale.

These power spectra show some structure at certain angles, as in the triangular wedge, so a simple horizontal or vertical lineout is not an adequate way of comparing them. A more meaningful comparison is possible by using the modulation transfer function (MTF), which has several definitions in the technical literature[65][71]. In this study the MTF is defined as a plot that shows the average frequency content versus spatial frequency for a particular power spectrum. The average is calculated by adding up the power of all the elements at a certain distance $r=\sqrt{\omega^{2}+\nu^{2}}$ from $(\omega, \nu)=(0,0)$ and then dividing by the number of elements at that distance. The MTF extends from zero to $1 / 2$ in spatial frequency regardless of the array or pixel size.

Figure 5.3 shows the MTF of the power spectra shown in 5.2. This plot de- 

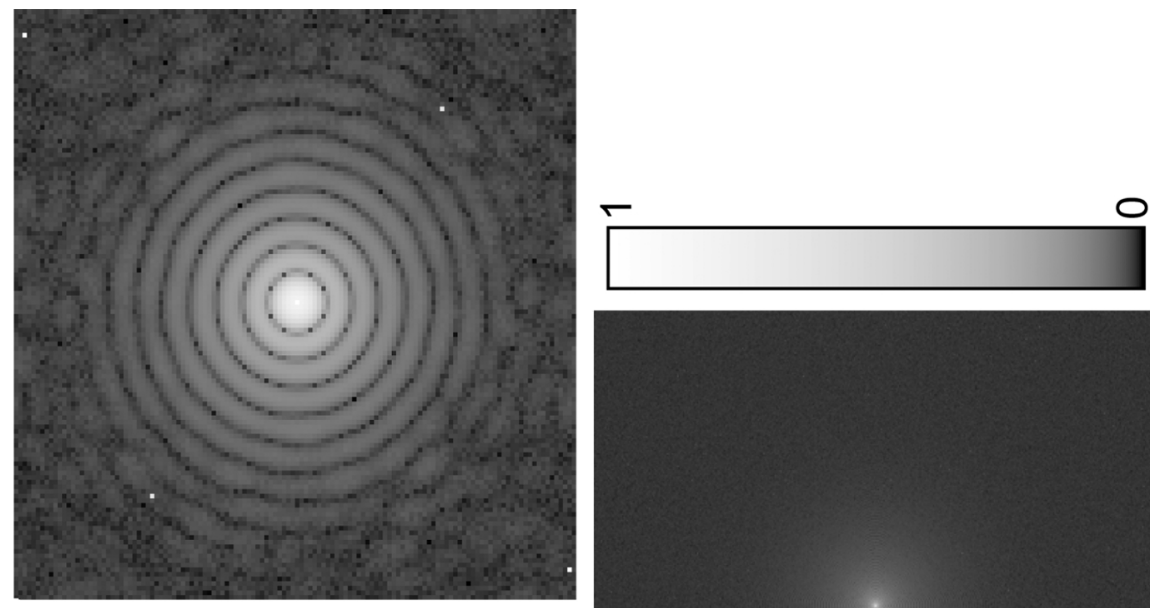

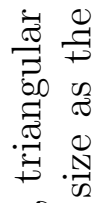
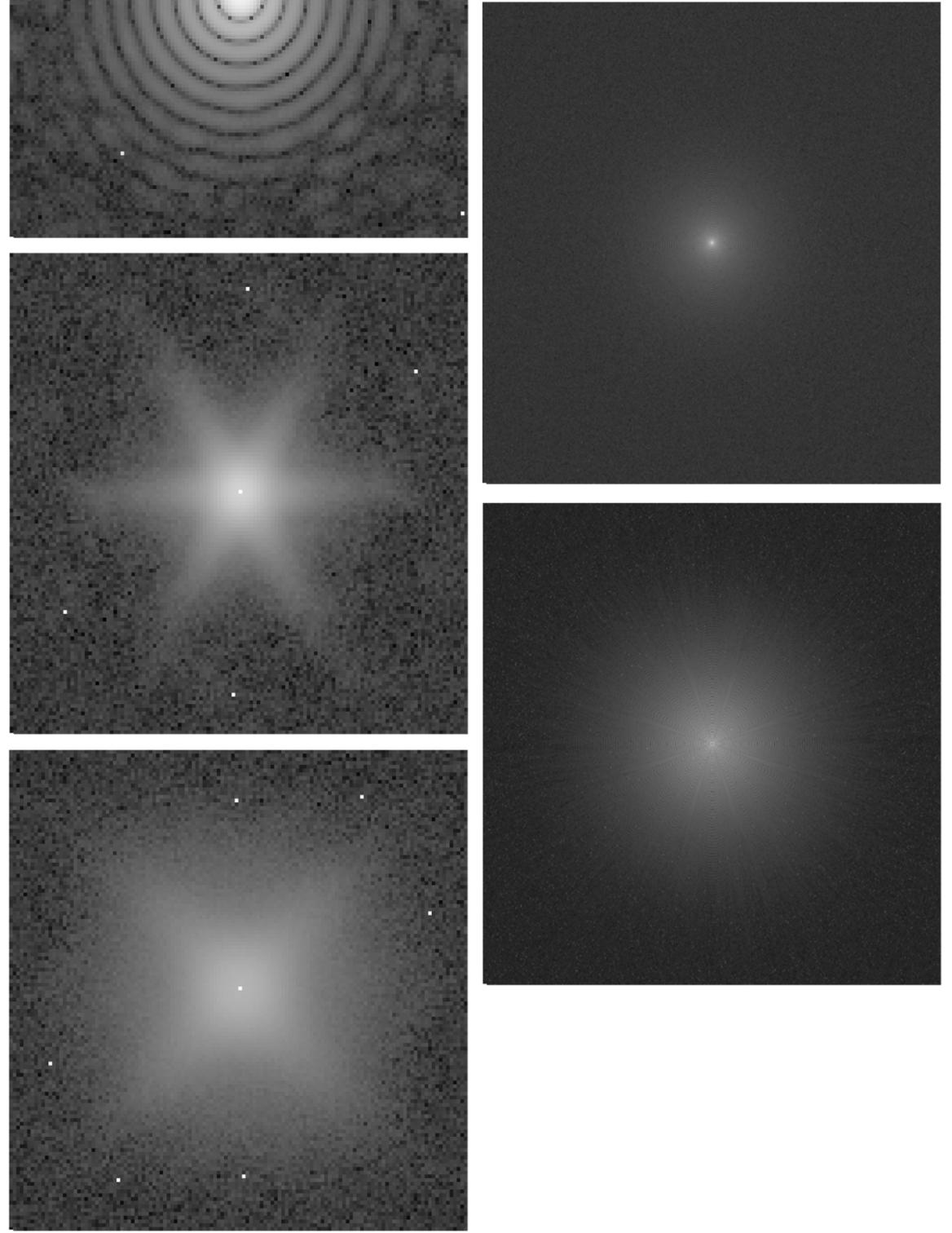

$\stackrel{0}{\circ} \stackrel{0}{0}$

.

0

哥

青㫄

잉ㅇ

त्र कि

$\pm \dot{0} . \exists$

峲

हैं 의

늘

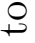

द्व

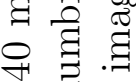

ॠ

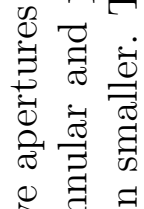

造寻

킁 ज

फ范

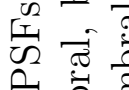

읰ㅋ

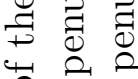

沾

苍

क 규유

¿. .્.

م.

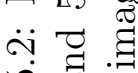

வ

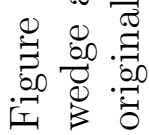




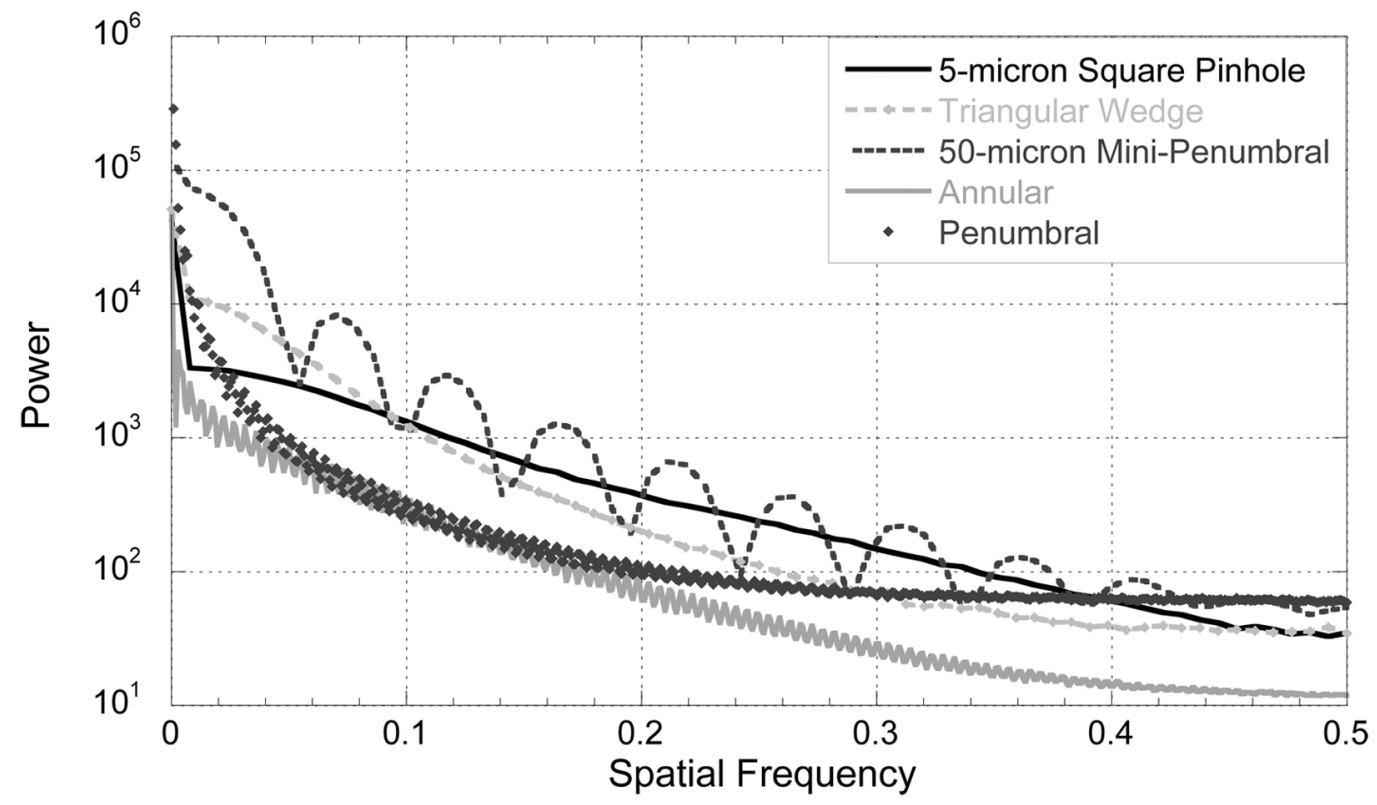

Figure 5.3: Modulation Transfer Function of the $40 \mathrm{~m}$ PSFs. Notice the high frequency modulation in the penumbral and annular apertures.

mostrates that all apertures transmit sufficient content at all frequencies to be useful in imaging applications. The mini-penumbral MTF is an interesting case since it shows a profile with some dips or notches, circular regions of low frequency content. This means that some frequencies are lost or poorly reproduced. This notch pattern is related to the size of the aperture; notice how the penumbral aperture has a similar distribution, but without the low frequency modulation. The extreme case of this modulation is the FFT of a point source, the two dimensional sinc function, which has an infinite number of oscillations.

The MTF of the difference downscattered images at $40 \mathrm{~m}$ are shown in figure 5.4, the power levels of the triangular wedge and mini-penumbral aperture have been 


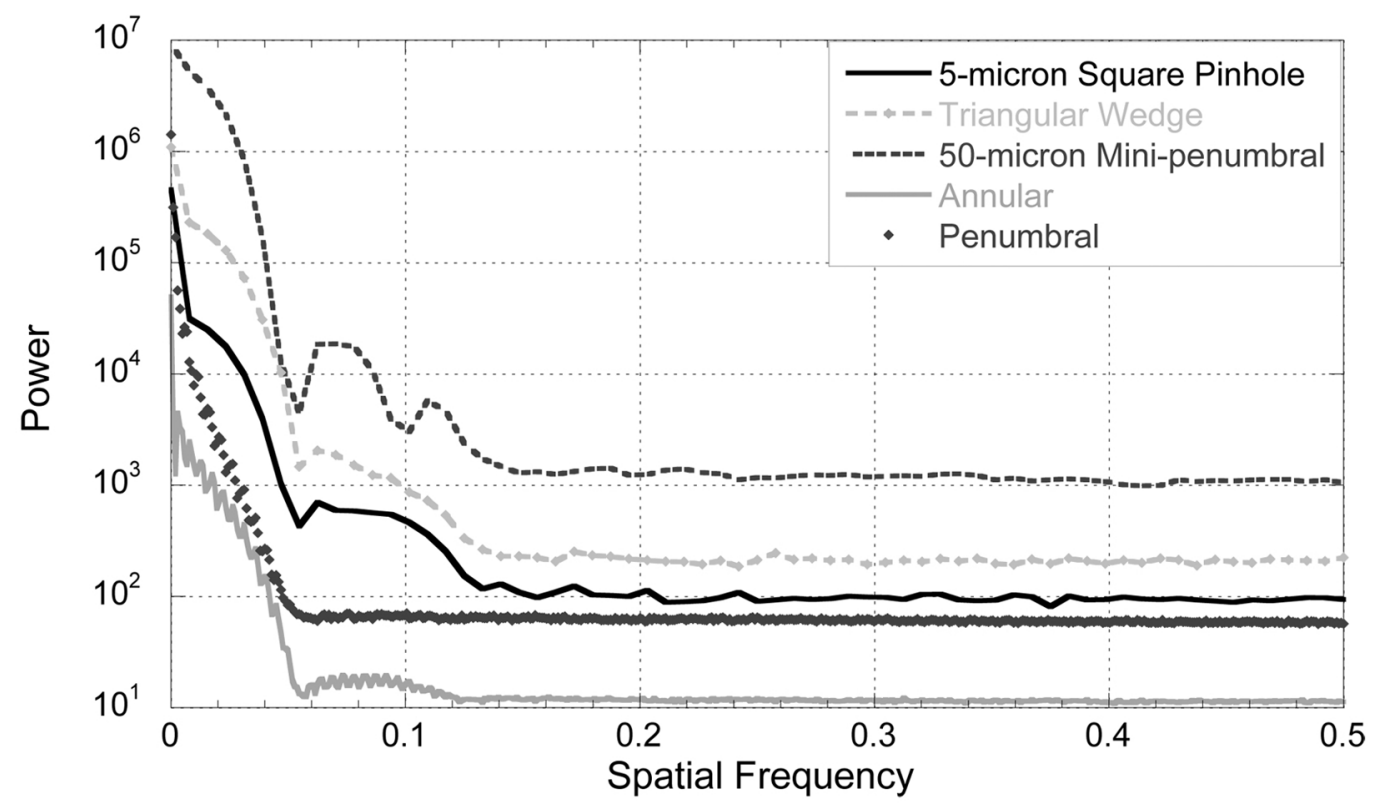

Figure 5.4: Modulation Transfer Function of the $40 \mathrm{~m}$ difference downscattered images.

adjusted so they do not overlap. These MTFs are similar to the PSFs ones, but they do not extend all the way to $1 / 2$, they reach a constant level, white noise, i.e., the same power level is present at all frequencies. After this point there is no more image information. The actual source information is encoded close to the center of the spectrum.

\subsubsection{Source Reconstruction}

The technical literature is abundant in image reconstruction methods or deconvolution algorithms[71][72][73][74][75]. This study uses a variant of the simplest method, direct inversion, in order to provide a straight forward method for comparing the dif- 
ferent apertures designs. An alternate method is presented for the penumbral and annular apertures to compare the results of the present study with the work done at CEA.

\subsubsection{Modified Regularization}

The simplest approach to source reconstruction is to use an inverse filter based on the corresponding PSF, as described in 5.25. This inversion is readily accomplished with the MCNPX generated PSFs, since their FFTs do not contain zeros, that are a common feature of ideal mathematical PSFs. For example, a series of tests showed that the reorganized FFTs of images where the direct transmission pedestal was replaced by a constant average value, contain zeros in the first column and row. The MCNPX arrays have high frequency content associated with the statistical variations between neighboring pixels.

The inverses of these PSFs contain very small values at high frequencies, which leads to amplification of the contents of $\operatorname{Im}(\omega, \nu)$ at these locations (see equation 5.25). The effects of direct inversion for the five micron square pinhole difference downscattered image is shown in figure 5.5, where the MTF of $O(\omega, \nu)$ is dominated by high frequency noise. A modified regularization was applied to $P S F(\omega, \nu)$ to mitigate the noise amplification. Regularization is the mathematical process in which zeros or elements beyond a certain point in the complex array are repalced by a constant. In the simplest form of regularization a constant is added to the whole array. 


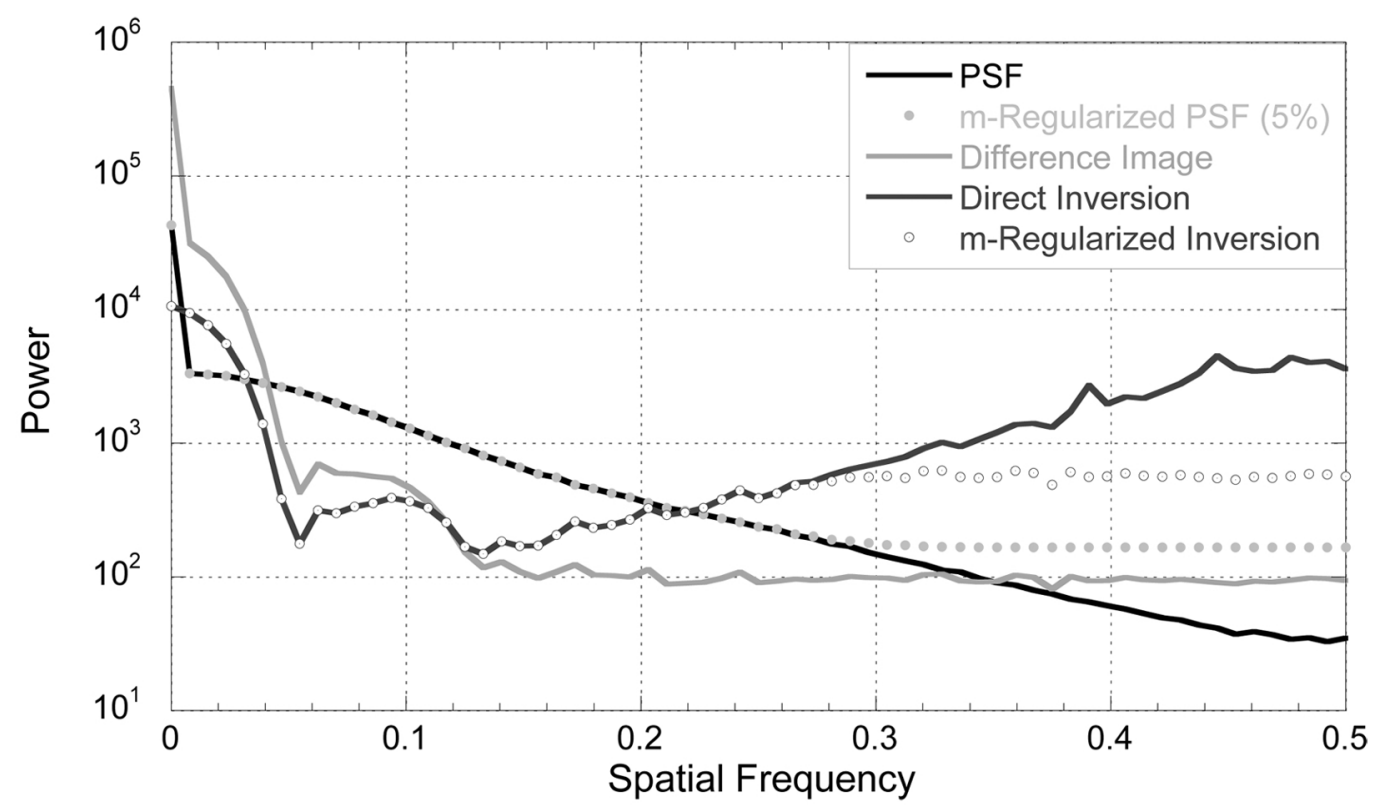

Figure 5.5: MTF of the 5-micron square pinhole difference downscattered source $(O(\omega, \nu))$ obtained through direct inversion and modified regularization.

In the modified regularization (m-regularization) approach there is no hard cutoff frequency or limit, but a minimum local frequency power $\left(|(\omega, \nu)|^{2}\right)$; any element with a power content below this limit is replaced by a constant, the regularization parameter, regardless of its location. This is done to preserve the frequency structure of the complex array as much as possible. For these elements, the m-regularization rule can be written as

$$
P S F^{\prime}(\omega, \nu)=P S F(\omega, \nu) \frac{\text { maximum } \times R P}{|P S F(\omega, \nu)|^{2}}
$$

where the regularization parameter $R P$ is defined as a percentage of the maximum ${ }^{4}$ of the corresponding power spectrum. $R P$ is different for each aperture and can be ad-

\footnotetext{
${ }^{4}$ The maximum in this context is not the value of $(\omega, \nu)=(0,0)$, but the average of the surrounding eight elements. This is done to enhance the dynamic range of the regularization parameter, since the PSFs have FFTs with a very high central peak.
} 
justed up to a certain extent. Values very close to $100 \%$ turn the PSF into a constant and no deconvolution is achieved; high values tend to erode the high frequency content and smooth out the images. Optimum results are obtained with values between $0.1 \%$ and $25 \%$. The MTFs of the m-regularized PSF and corresponding recovered source are shown in figure 5.5. With regularization, the high frequency noise does not increase as fast as before. The MTF of the m-regularized inversion does not have a pronounced DC peak, which means that the source is recovered without a direct transmission pedestal. This is a particularly interesting consequence of using a PSF of the same size as the recorded image.

Figure 5.6 shows the real space versions of the direct inversion and m-regularization recovered sources. The elements of these arrays are real numbers, both positive and negative. This seems to violate the requirement of an imaging algorithm to produce only positive valued pixels, but a closer examination of the arrays shows that the negative pixels are located outside the region occupied by the recovered source, that is, where the direct transmission pedestal used to be. In fact the histogram of this region has a mean very close to zero.

\subsubsection{Filtered Auto-Correlation}

This alternate image reconstruction method has been pursued successfully by Disdier et.al. with both penumbral and annular apertures[49]. This method was originally developed by Barrett et.al.[71][76] for x-ray imaging and later retooled by 

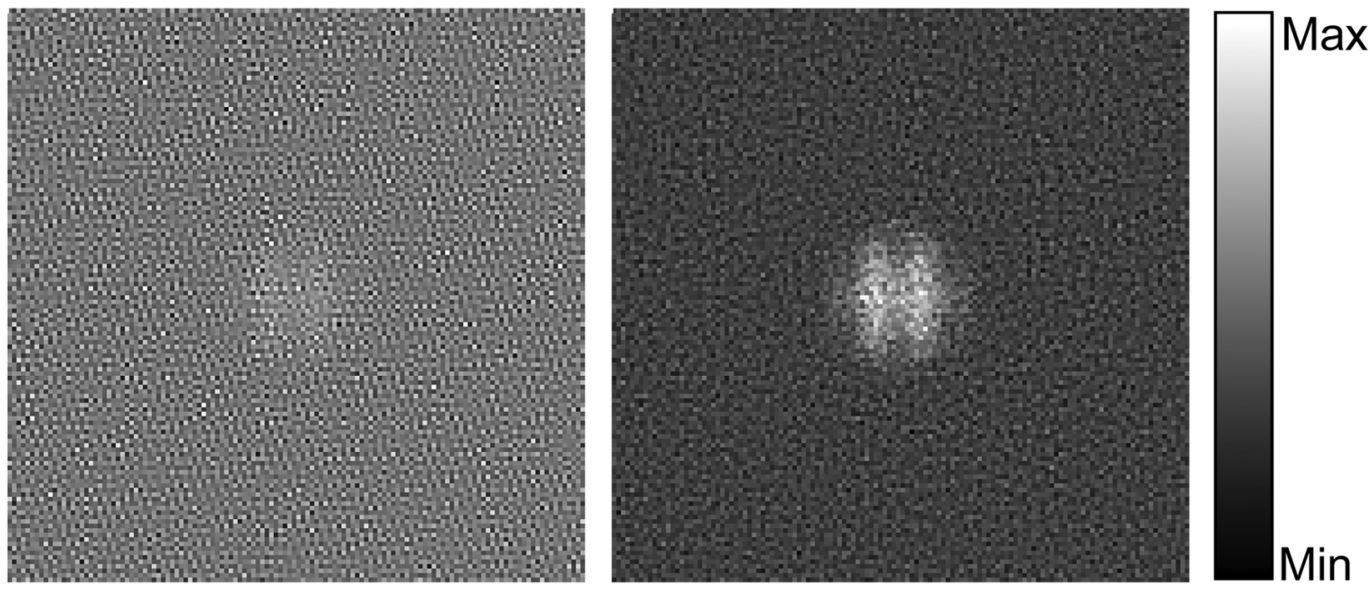

Figure 5.6: Real space plots of the recovered downscattered source for the 5-micron square pinhole. The direct inversion source (left) is barely visible due to the high frequency noise contamination. The m-regularized reconstructed source is distinguishable amidst the high frequency noise. The images represent a 320 microns square FoV. The normalized linear grayscale includes the negative valued pixels.

Rouyer[77] for neutron imaging. The main advantage of this method is that it can be modeled accurately by using the correlation operation and the symmetry and periodicity of circular functions in frequency space. The correlation operation (also known as cross correlation) in real space is given by[65]

$$
h(x, y) * * g(x, y)=\int_{-\infty}^{\infty} \int_{-\infty}^{\infty} h\left(x^{\prime}, y^{\prime}\right) g\left(x^{\prime}-x, y^{\prime}-y\right) \mathrm{d} x^{\prime} \mathrm{d} y^{\prime}
$$

and it can be shown that

$$
F\{h(x, y) * * g(x, y)\}=H(\omega, \nu) G(-\omega,-\nu)
$$

Correlation decoding can be seen as the process by which an encoded image is matched with a very specific mask or template so only the original source elements are obtained. By multiplying the raw image in frequency space by a special decoding 
function, the array of most likely original source elements can be obtained.

In correlation decoding of an annular or penumbral image, each point of the source array is replaced by a circle or disc of fixed radius $\rho$. The intersection of these new circles or discs represents the original source distribution. The more points in the coded image that are related to the source, the more intense the intersection locus will be compare to the rest of the image. This is a property of the autocorrelation function $(h(x, y)=g(x, y))$ that is frequently used in matched filtering to identify or detect particular features in an image.

It can be shown that the decoding function for a penumbral aperture is given by[77]

$$
2 \pi^{4} \rho^{3} s^{3} \frac{J_{1}(2 \pi s \rho)}{\pi s \rho}
$$

and for an annulus by[71]

$$
2 \pi^{3} \rho^{2} s^{2} \frac{J_{0}(2 \pi s \rho)}{\pi s \rho}
$$

where $\rho$ is the average radius, in pixels, of the penumbra or ring in the recorded image. $s$ is the radial spatial frequency, which for a reorganized $1024 \times 1024$ array is given by

$$
s=\sqrt{\left(\frac{512-j}{1024}\right)^{2}+\left(\frac{512-k}{1024}\right)^{2}}
$$

where $j$ and $k$ indicate the pixel's row and column.

Notice that $\rho$ is the only adjustable parameter in 5.31 and 5.32. $\rho$ has to be measured from the raw image ${ }^{5}$. Figure 5.7 shows the power spectra of the penumbral and

\footnotetext{
${ }^{5} \rho$ is determined by trial and error inspection. Wrong values of the radius will produce a recon-
} 

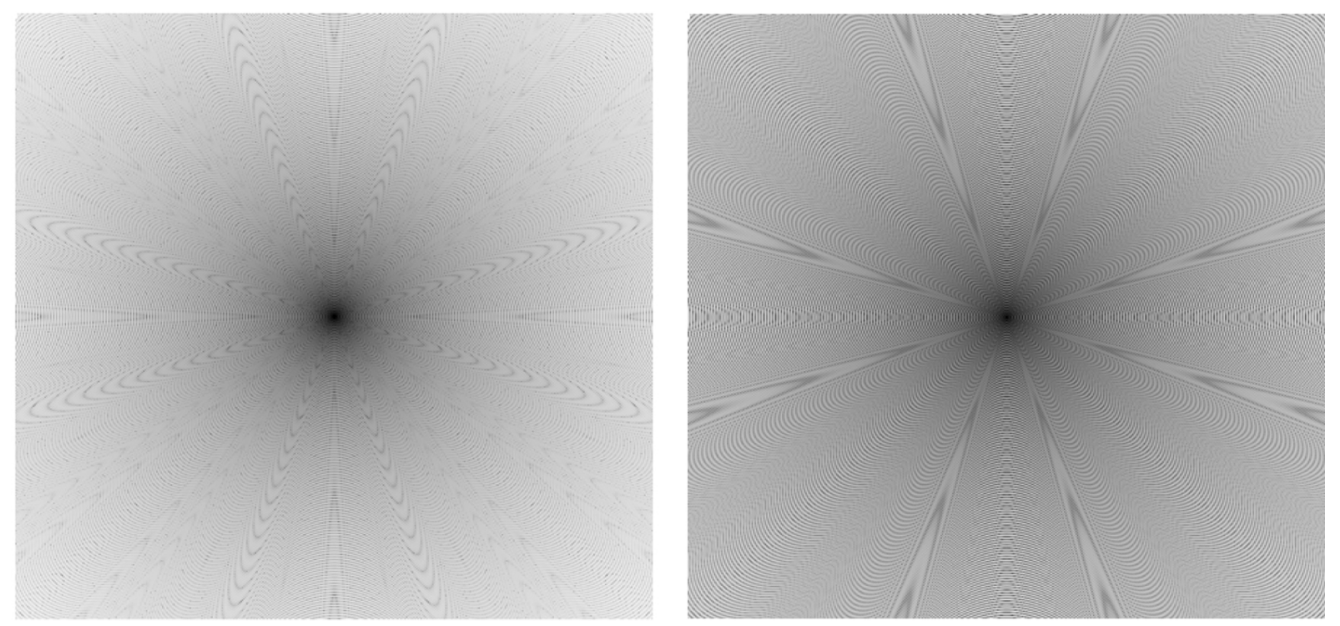

Figure 5.7: Power spectra of the penumbral (left) and annular decoding functions. The images are shown in a logarithmic grayscale. The radial structure in the images are aliasing artifacts and Moiré patterns.

annular decoding functions for the $40 \mathrm{~m}$ LOS; $\rho$ was obtained from the corresponding simulated PSFs.

No a priori knowledge of the system's PSF is required, a great advantage over the m-regularization method. At the same time, since the decoding function is based on a perfectly opaque ring of radius $\rho$, it ignores any special characteristics of the aperture or any misalignments of the system. Another advantage of this method is that it does not amplify the high frequency noise as much as the m-regularization, because no division in frequency space is required. The noise content of the reconstructed source is set by the noise in the raw recorded image only.

The MTF for the autocorrelation reconstructed downscattered annular source is shown in figure 5.8. The real space annular and penumbral sources at $40 \mathrm{~m}$ appears structed source that looks 'out of focus'. 


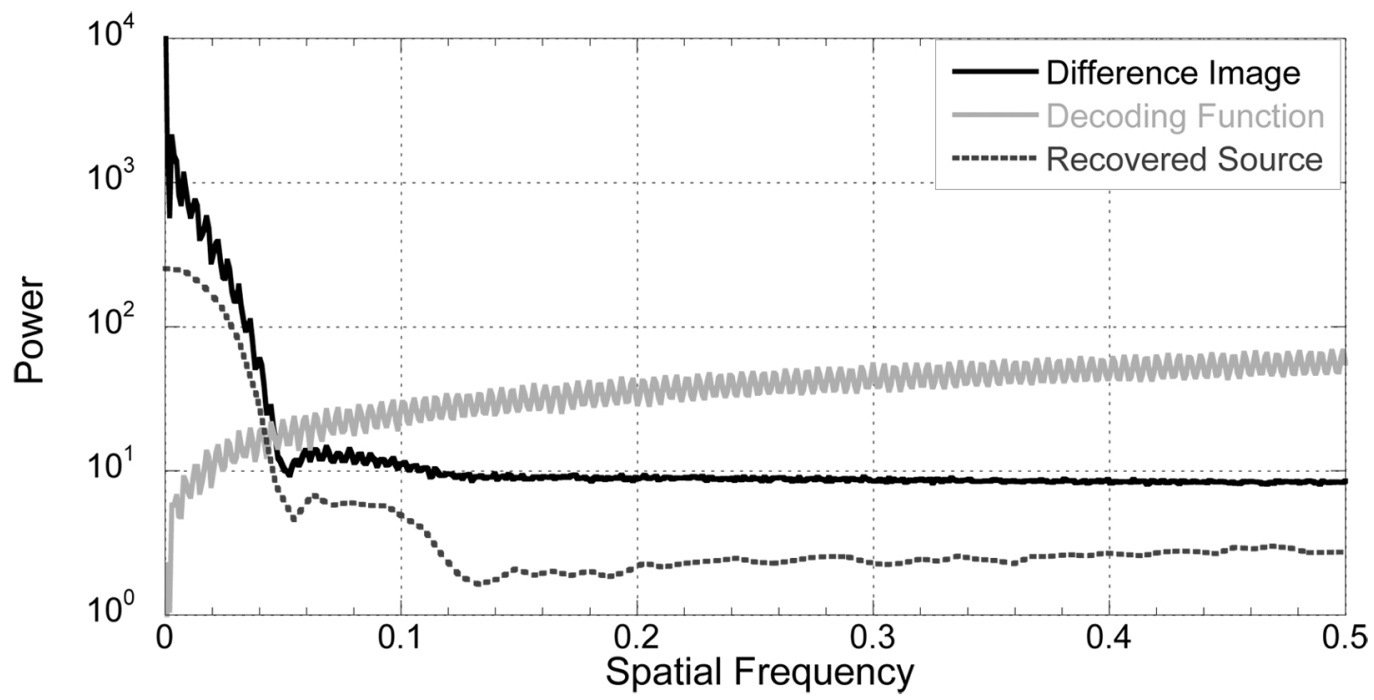

Figure 5.8: MTF of the annular downscattered source recovered using filtered autocorrelation. Notice that the recovered source has a very flat MTF.

in figure 5.9. As with the m-regularization images additional filtering is required.

The mathematical model used in the derivation of this method requires the aperture to be much larger than the object being imaged. However, the autocorrelation method was used to recover the sources for the mini-penumbral apertures, generating a usable image with the 50-micron aperture, while marginal results are obtained with a 100-micron aperture. In both cases the direct transmission pedestal is not completely eliminated and additional filtering is necessary (see figure 5.10).

\subsubsection{Low-Pass Filtering}

The high frequency noise present in the deconvolved images can be attenuated by applying a low-pass filter in frequency space. 

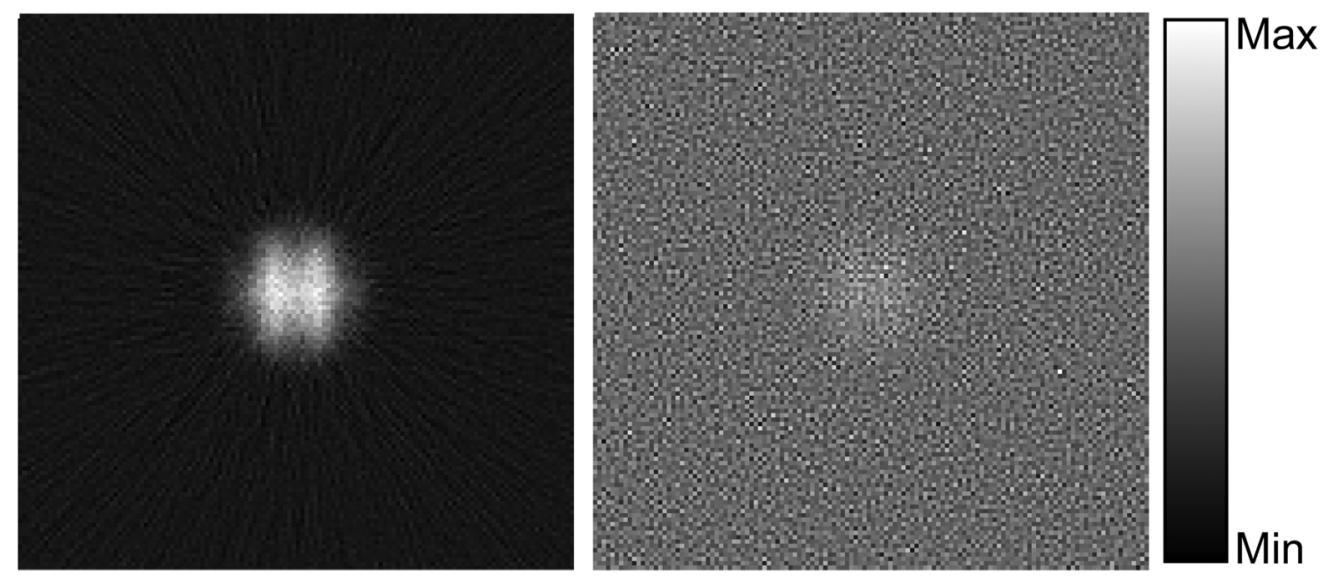

Figure 5.9: Downscattered source recovered using filtered autocorrelation for the annular (left) and penumbal apertures. The annular source looks very good and shows a low noise level. The penumbral source is almost lost behind the high frequency noise. The images have been cropped to 128 microns and the grayscale has been extended to account for negative values.

A Gaussian-shaped filter offers the advantages of ease of implementation and very low level of ringing in the reconstructed source[69]. The normalized filter array was generated in real space using the expression[69]

$$
R(j, k)=\frac{1}{2 \pi \sigma^{2}} \exp \left(\frac{-\left[\left(j-\frac{L}{2}\right)^{2}+\left(k-\frac{L}{2}\right)^{2}\right]}{2 \sigma^{2}}\right)
$$

where $L$ is the size of the array (128 or 1024), and the standard deviation $\sigma$, in pixels, is obtained from the filter's FWHM, in microns, using

$$
\sigma=\frac{\mathrm{FWHM}}{2 \sqrt{2 \ln (2) P}}
$$

where $P$ is the size of the source pixel depending on the system's magnification (based on 250 micron fibers: 2.5 microns/pixel for $40 \mathrm{~m}$ LOS, 3.57 microns/pixel for the $28 \mathrm{~m}$ LOS). The filter is then transformed using cfft and multiplied by the reconstructed 

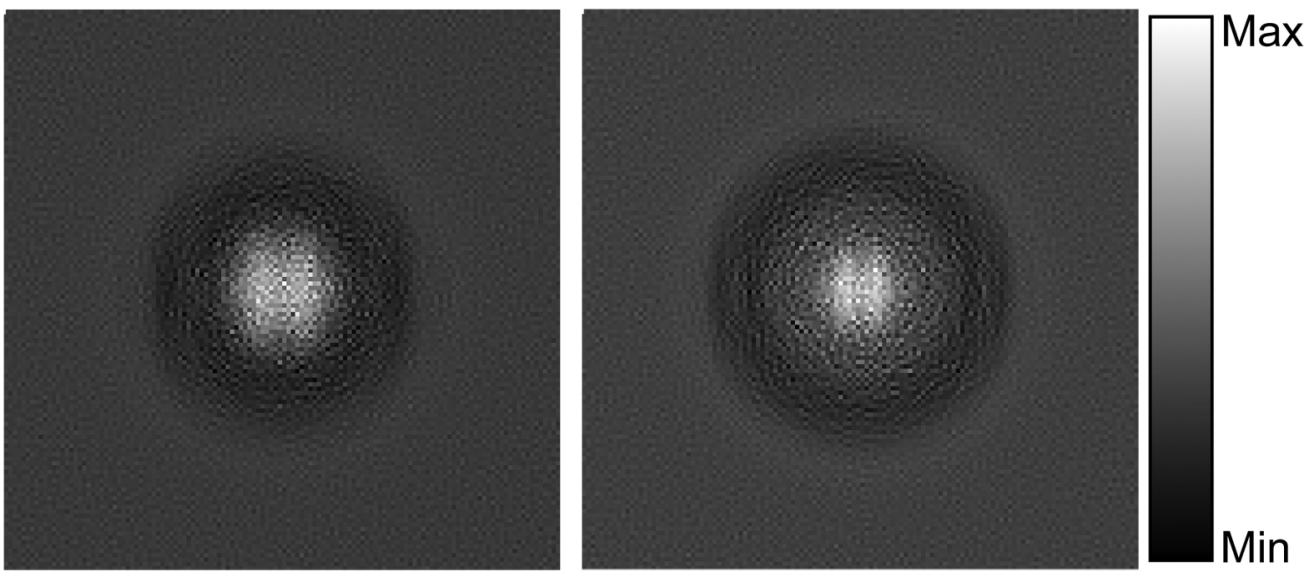

Figure 5.10: Downscattered source recovered using filtered autocorrelation for the 50-micron (left) and 100-micron mini-penumbral apertures. The direct transmission pedestal is only completly eliminated in a narrow region around the source. In the 100-micron case the source still has a small pedestal attached to it. Complete $128 \times 128$ array shown.

source array. The resulting array is inverted using icfft and is ready for display.

The optimum value of FWHM can be determined by inspection of the MTF of the raw image. The purpose of the filter is to cancel out as much of the high frequency noise as possible without eliminating any source information from the image. Figure 5.11 shows the effect of several filters on the the MTF of the reconstructed downscattered source for the 5-micron square pinhole, along with the corresponding real space images (cropped to 100 microns). It can be seen that the wider the filter, the smoother the source becomes, but more information is lost. The optimum character of the filter's width is based on the visual comparison of the original source with the reconstructed array. In Chapter Six numerical values are associated with the concept of image quality. The optimum FWHM of the filter varies between 7 and 20 microns. 

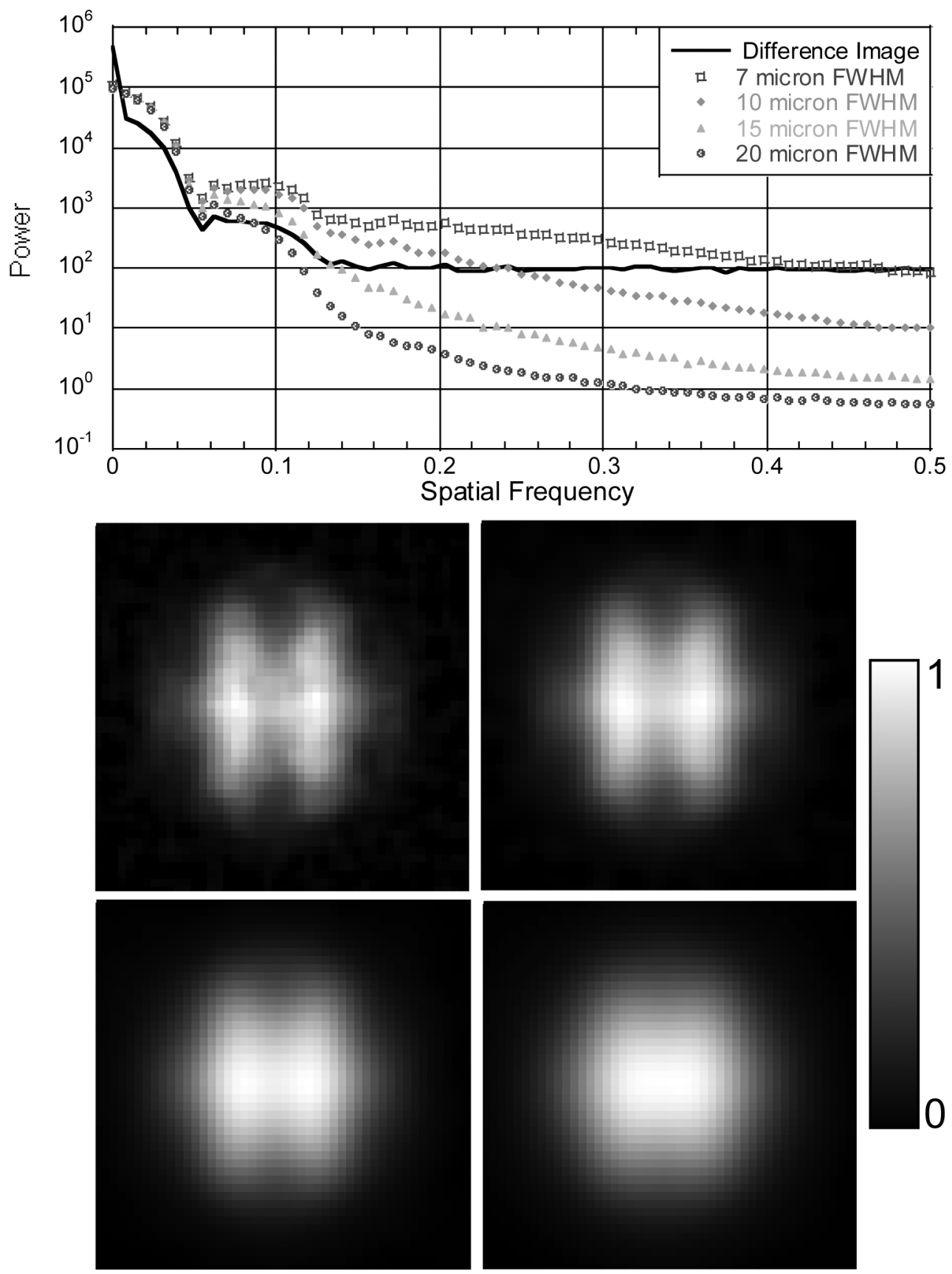

Figure 5.11: MTF of the 5-micron square pinhole recovered sources after a low-pass Gaussian filter. The real space images are cropped to 100 microns and correspond to 7 microns (top left), 10 microns (top right), 15 microns (bottom left) and 20 microns FWHM. 

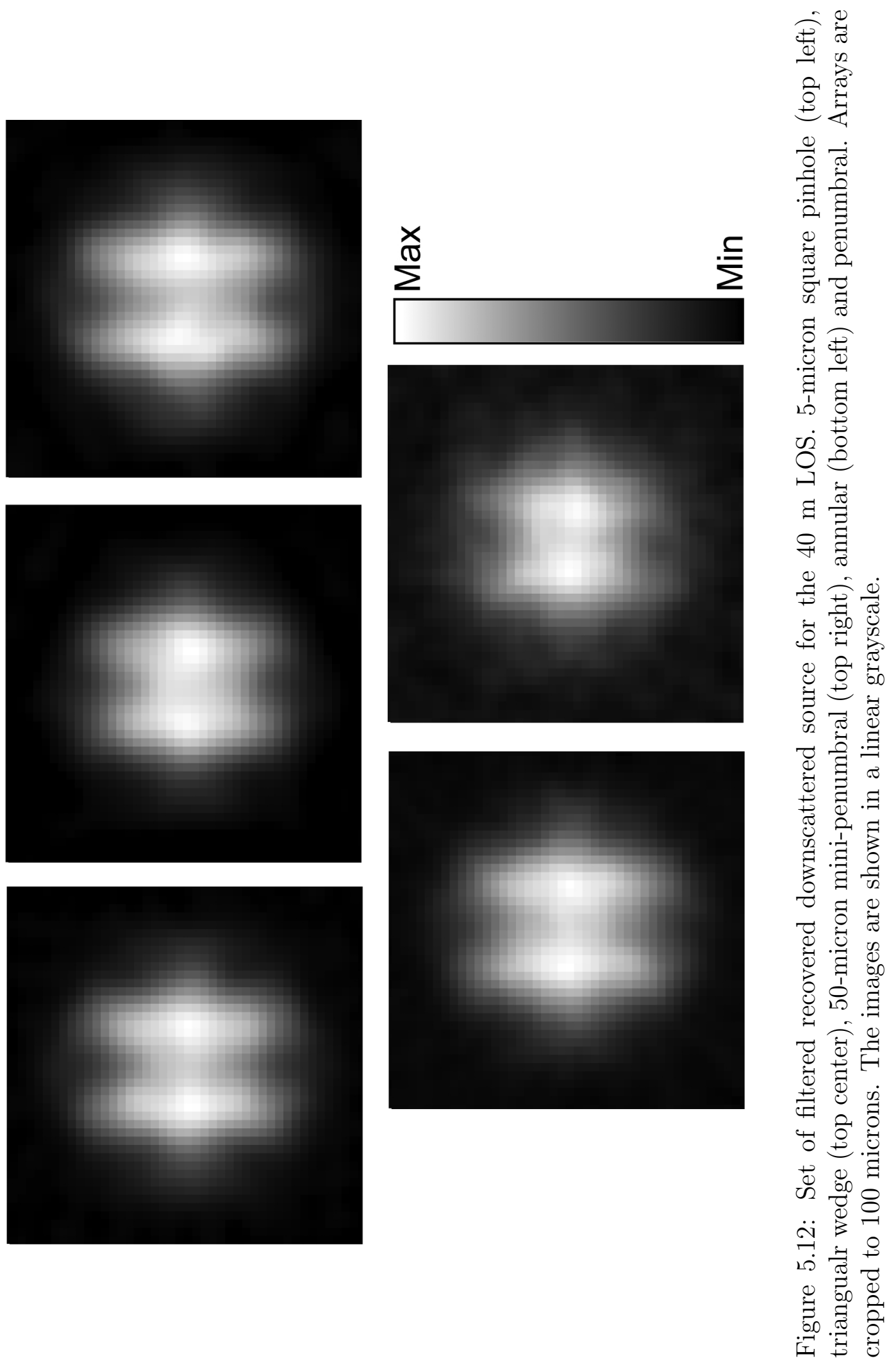


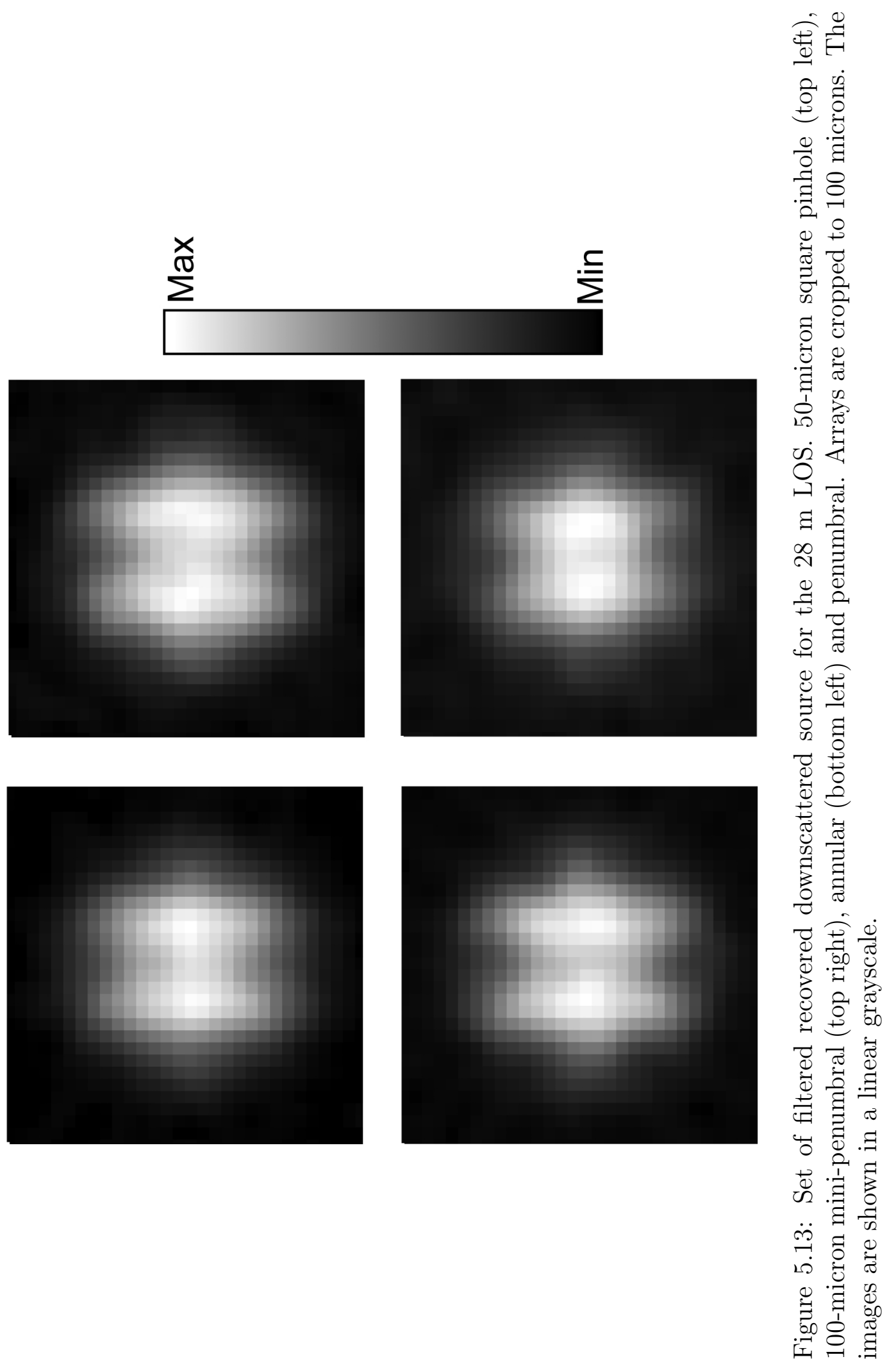


Figures 5.12 and 5.13 show the final processed downscattered images for the 40 and $28 \mathrm{~m}$ lines of sight respectively.

The Gaussian filter is by no means the only alternative available for the NIS post-processing. It is important to further explore other shapes like super Gaussians, which have a wider flat top, and matched filters that are known to work well with aperture arrays[71].

\subsubsection{Field of View Variation}

Both reconstruction techniques presented here are based on the isoplanatic approximation and thus they only use an on-axis PSF or decoding function. It is important to verify that the reconstructed primary and difference images are accurate. The FoV intensity response map of each aperture was generated using MCNPX. The FoV was sampled using a uniform intensity 150-micron diameter disc-shaped source placed at TCC. The equivalent $8 \mathrm{MeV}$ neutron yield was $10^{16}$. The recorded images were deconvolved using the m-regularization method with the same parameters of the real sources.

Figure 5.14 shows the average pixel value as a function of radius for the $40 \mathrm{~m}$ LOS, figure 5.15 shows the results for the $28 \mathrm{~m}$ LOS. Each aperture has a different FoV profile; most show a constant intensity for $r<10$ microns and then drop off slowly to $70 \%$ at $r \approx 50$ microns due to vignetting effects.

Exceptions to this are the triangular wedge, that shows a flat profile through 


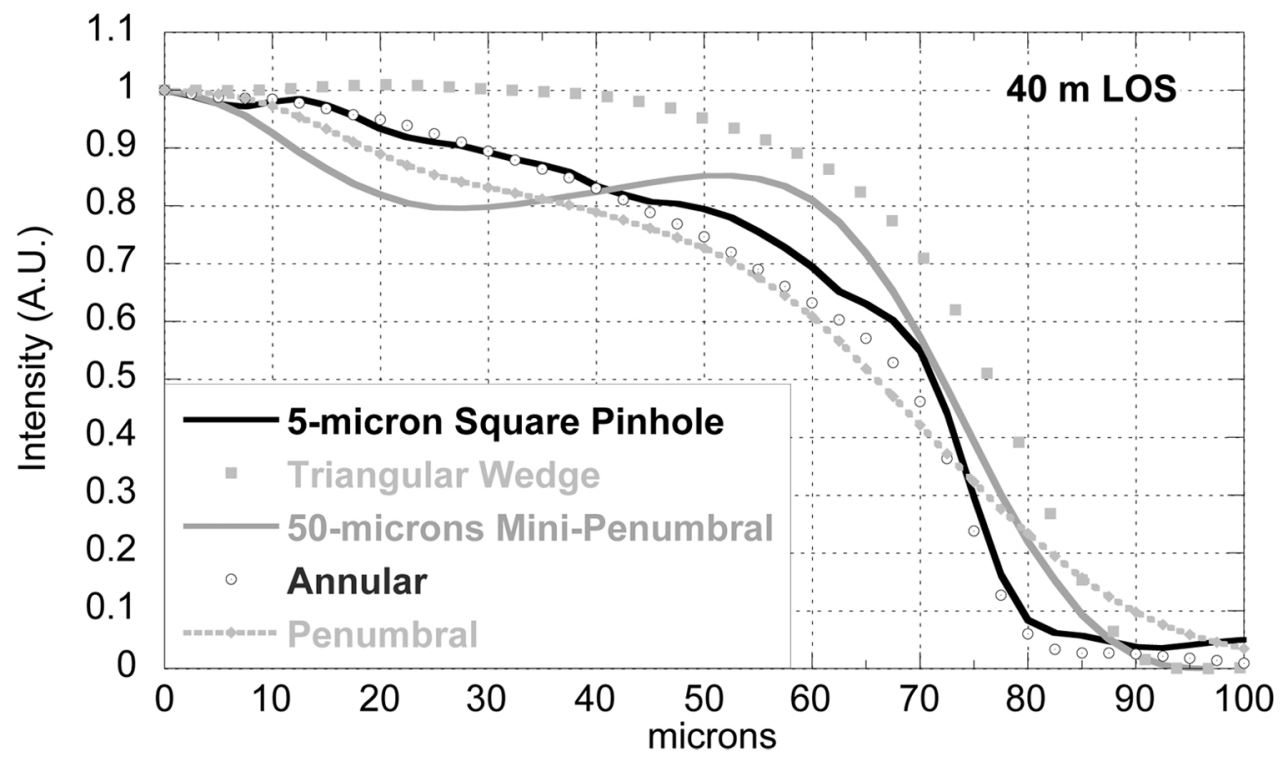

Figure 5.14: Intensity variation across the FoV for the $40 \mathrm{~m}$ LOS.

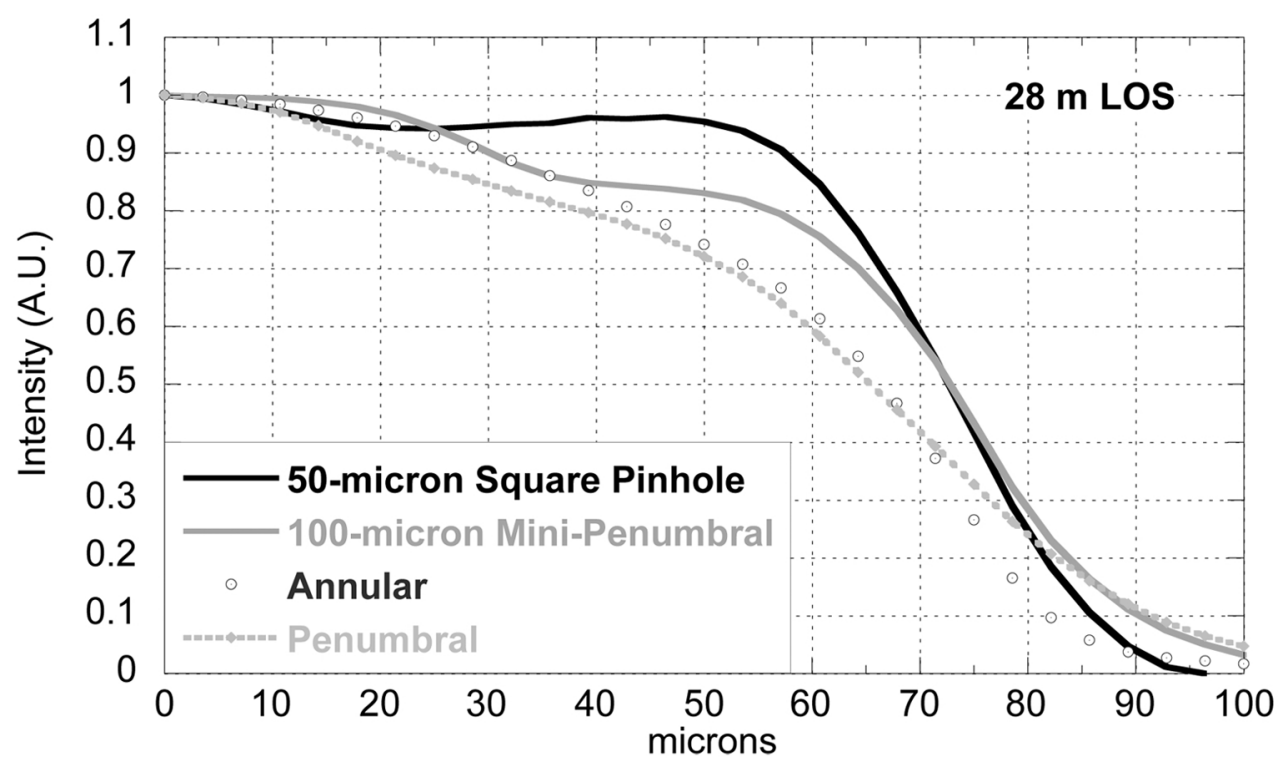

Figure 5.15: Intensity variation across the FoV for the $28 \mathrm{~m}$ LOS. 
$r=40$ microns, and the 50-micron square pinhole. Both mini-penumbral apertures show an intensity oscillation, the 50-micron case being more pronounced between 25 and 50 microns. The 100-micron mini-penumbral shows a small plateau and then rolls off at $r=30$ microns.

The expected size of the compressed cores during NIC experiments is 80 to 100 microns in diameter. In general, the reconstructed primary sources do not need an additional correction due to FOV intensity variations, but additional compensation might be necessary for the downscattered sources and misaligned targets or bigger cores that fall outside the central area. The intensity correction can be made in real space by multiplying the final filtered reconstructed source by the inverse of a normalized array base on the FoV intensity profiles of figures 5.14 and 5.15 .

Another FoV uniformity test consists of placing small bright sources at increasing distance from the center and deconvolving the image using the on-axis PSF. Figure 5.16 shows the raw image and deconvolved source of four 10-micron discs placed at $(0,0),(0,20),(40,0)$ and $(40,40)$ microns and imaged through the 50-micron square pinhole. The reconstructed discs appear at the right locations and show no major distortions in shape or intensity. 

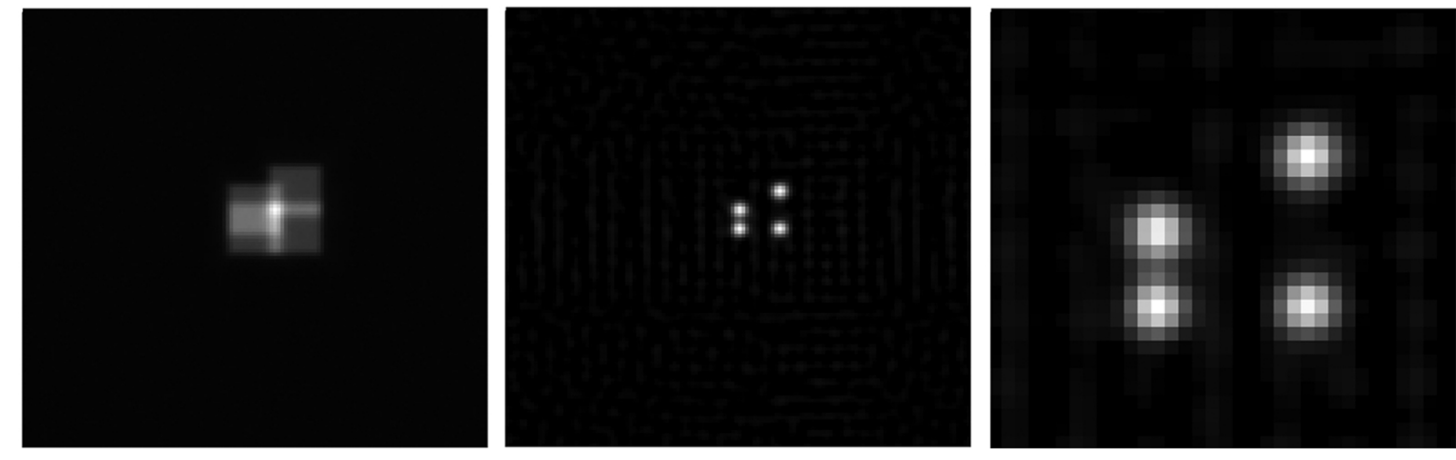

Figure 5.16: Four 10-micron discs imaged with a 50-micron square pinhole and a 28 $\mathrm{m}$ LOS. Raw recorded image(left), full array m-regularized source (center), source cropped to 110 microns. 


\section{Chapter 6}

\section{Image Quality}

The processed receovered sources obtained using the NIS end-to-end model and the m-regularization process can be used to compare and understand the imaging capabilities of the different LOS configurations. The images presented at the end of chapter five (figures 5.12 and 5.13) show that all the configurations under consideration are capable of producing a downscattered neutron source, but in order to make an informed decision about the optimum NIS design, an image quality metric has to be used.

This chapter presents a discussion on image quality from the point of view of the observer and compares it with the NIS design requirements. The statistics of noise and two additional parameters (resolution and signal-to-noise ratio) to measure the quality of the recovered sources. These parameters are evaluated for the LOS configurations under consideration, using a special MCNPX source. 


\subsection{Image Quality and Design Requirements}

The ultimate effectiveness test of a LOS configuration/source recovery algorithm combination is to produce an image that can be correctly interpreted by a trained observer. The output image is useful if it contains enough information so that the observer can decide, with a high degree of confidence, whether a particular feature is present or not. Imaging assessments often divide into four categories the judgments that a trained observer can make about an image:

- True Positive: observer says feature is present in the image when it actually is

- True Negative: observer says feature is not present when it is absent

- False Positive: observer says feature is present when it is absent

- False Negative: observer says feature is absent when it is actually present

An imaging system is said to be effective when the judgments of an unbiased trained observer are mostly true positives and true negatives. The observer's interpretation of an image is not limited the presence or absence of a feature; it also deals with its location, size and relation with the rest of the image. An image is said to be of good quality when the feature of interest is rendered with sufficient detail and it is distinguishable from its surroundings.

The level of detail is associated with, among other things, with the dynamic range of the imaging system, the physical size of the feature, and the shape and size 
of the pixels. These in turn depend on the system's resolving power, the optical magnification, and the detector's geometry.

The contrast level within and around the feature of interest allows the observer to distinguish any internal structure, to separate it from secondary features, and from the background. The contrast level is associated with the dynamic range of the imaging system, and the noise and background levels. The contrast depends on the signal-to-noise ratio (SNR), where the signal is the portion of interest of the image, and the noise is related to the non-image content and its intensity distribution.

In both of the above contexts the dynamic range refers to the system's ability to record useful images over a wide range of intensities; this means the detector has to be sensitive enough to work at the lowest and not saturate bellow or at the maximum intensities. The image recording system, usually a CCD camera, also must have sufficient enough color depth (pixel data bits) to distinguish between very close light intensities.

The optical magnification, size and distribution of the pixels, and over-sampling at the CCD camera are interrelated and must be considered for a correct treatment of image resolution. It is imporatant to note that for imaging purposes the actual quantity of interest is the resolution element, which can be made of one or multiple pixels. For the NIS the size of the resolution elements often will dictated by the size of the detector scintillator fibers, but the optical system and the camera will change this size and over-sample the fibers due to the very small size of the CCD pixels. 
The detector array in some cases can not be mapped into simple square pixels. For example the CEA liquid filled fused silica capillaries, are a close packed hexagonal array. In general, the resolution element is not equal to the recorded image pixel.

For this study and for the design of the NIS, the two main parameters used to quantify the quality of an image are resolution and SNR. The design requirements state that the NIS must have a resolution of 10 microns and a SNR of 10 at the $20 \%$ contour, but no specific definition of either term is given. Before studying these two parameters in detail, it is important to discuss the noise characteristics of the expected neutron images.

\subsection{Image Noise}

In neutron imaging, as in other radiological imaging techniques, the actual image is formed by the interaction of individual particles, the neutrons, with a detector and recording system. The noise in the image is a manifestation of the actual number of particles that interact with a detector element; the noise is the range of recorded values generated from a constant source. This noise is associated with counting statistics.

In a neutron image additional noise sources include direct neutron interaction on the CCD (stars), electronic noise, and scattered radiation, particularly activation $\gamma$ rays reaching the detector or scattered high energy neutrons. The modeling of these sources is highly dependent of the final geometry of the NIS and its location at the NIF, and it is beyond the scope of the present study. 
The counting statistics have a Poisson distribution[34], which is given by

$$
P(x)=\frac{(\bar{x})^{x} \exp (-\bar{x})}{x !}
$$

where $\bar{x}$ is the mean value and the standard deviation is equal to $\sqrt{\bar{x}}$. In counting experiments the mean is equal to the number of counts. The modeling of noise level in the image is complex since it depends on the actual image intensity, not just on external sources. For example, the widely used Wiener inverse filter, which in full form is given by[69]

$$
R_{W}(\omega, \nu)=\frac{1}{P S F(\omega, \nu)}\left[\frac{|P S F(\omega, \nu)|^{2}}{|P S F(\omega, \nu)|^{2}+\frac{N(\omega, \nu)}{\operatorname{Im}(\omega, \nu)}}\right]
$$

where $N(\omega, \nu)$ is the noise kernel, can not be directly used in neutron imaging, since the counting noise does not have the well defined kernel of electronic or film detectorso.

The noise in neutron imaging has to be studied for each contribution to the image; from the number of neutrons entering a fiber in the detector, to the number of photoelectrons emitted by the MCP photocathode, passing through the number of recoil protons produced by a neutron, their energy deposition and the number of photons produced by the scintillator that make it into the MCP.

The MCNPX end-to-end model was used to study the image statistics at each stage of the image formation. The direct transmission pedestal provides a large pixel sample histogram, that can be fitted to a Poisson or Gaussian distribution. Figure 6.1 shows several histograms of the 5-micron square pinhole direct transmission. The quantities per pixel are: neutrons entering, neutron collisions, $\mathrm{MeV}$ deposited and 
photoelectrons produced.

These histograms show that both the number of neutrons entering per pixel and neutron collisions per pixel follow a Poisson distribution. The other two quantities are close to being Gaussians, but in reality they are convolutions of the original Poisson with other probability distributions. For example the energy deposited per pixel, is a convolution of the $(n, p)$ elastic scattering cross section with the proton range and stopping power. These simulations confirm, as described originally by Moran et.al.[78], that the image noise is dominated by the number of neutrons entering a detector element.

Figure 6.2 shows the histogram of the pedestal region for the corresponding reconstructed source: first just the m-regularized inversion result and then after a 15 micron FWHM low-pass filter. The distribution of the unfiltered reconstructed source is extremely wide compared to the original photoelectrons per pixel, because of the inversion in frequency space and the associated noise amplification. In these cases the distribution is clearly Gaussian. The Poisson fit is not shown becasue the high value of the mean produces a distribution that looks very narrow. The low-pass filter narrows the distribution, lowering its standard deviation $\sigma$. The calibration of the pixel intensities is not clearly defined in these cases, since the inversion step destroys the normalization of the image. It is important to study further the nature of the image after deconvolution, since a final reconstructed source calibrated in terms of primary neutrons per source element would be very useful. 

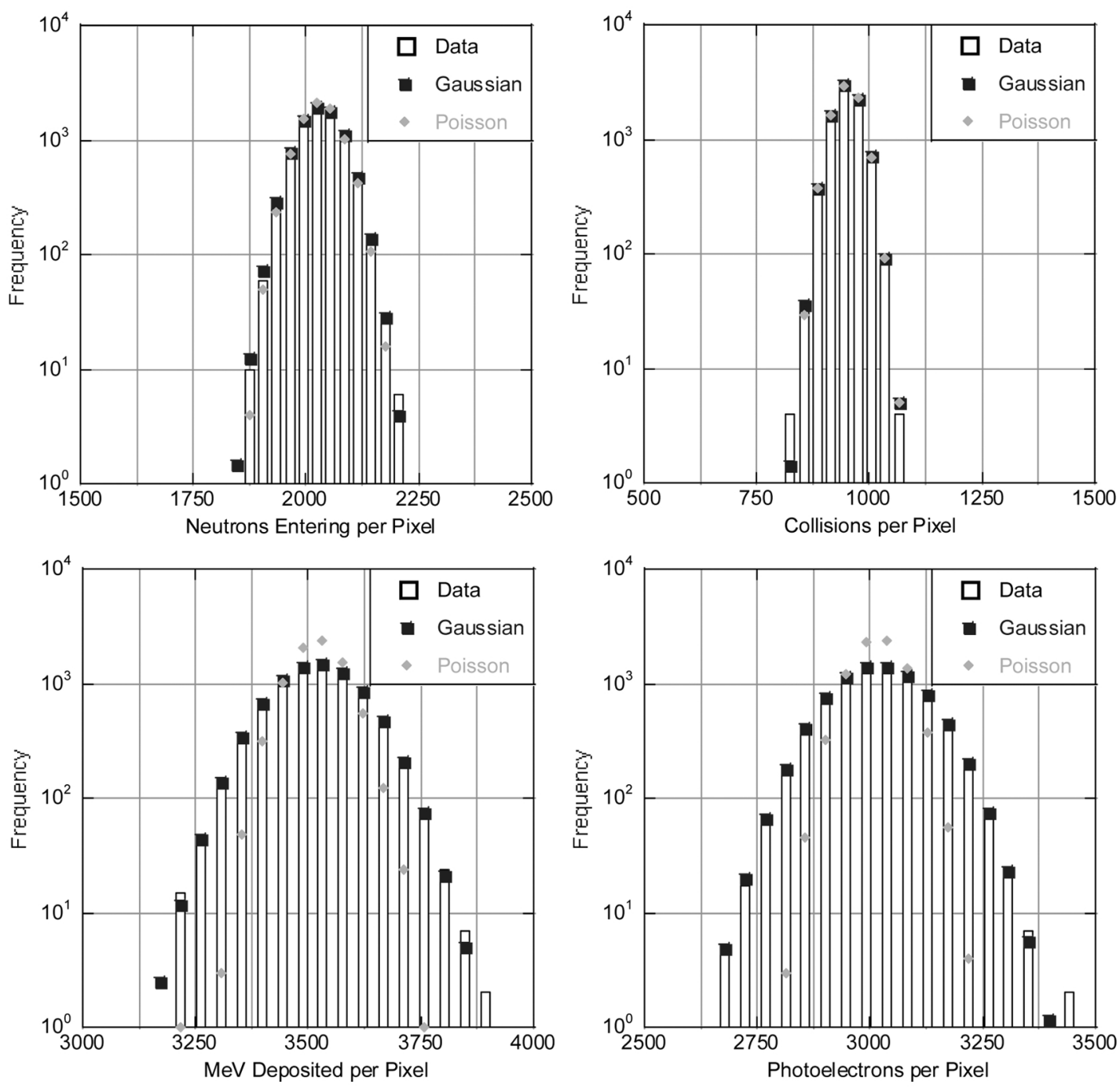

Figure 6.1: Histograms of the direct trasnmission pedestal of the 5-micron square pinhole. The equivalent source intensity is $10^{16} 8 \mathrm{MeV}$ neutrons. The quantities per pixel have feen fitted to Gaussian and Poisson distributions with the same mean and standard deviation of the original data. 

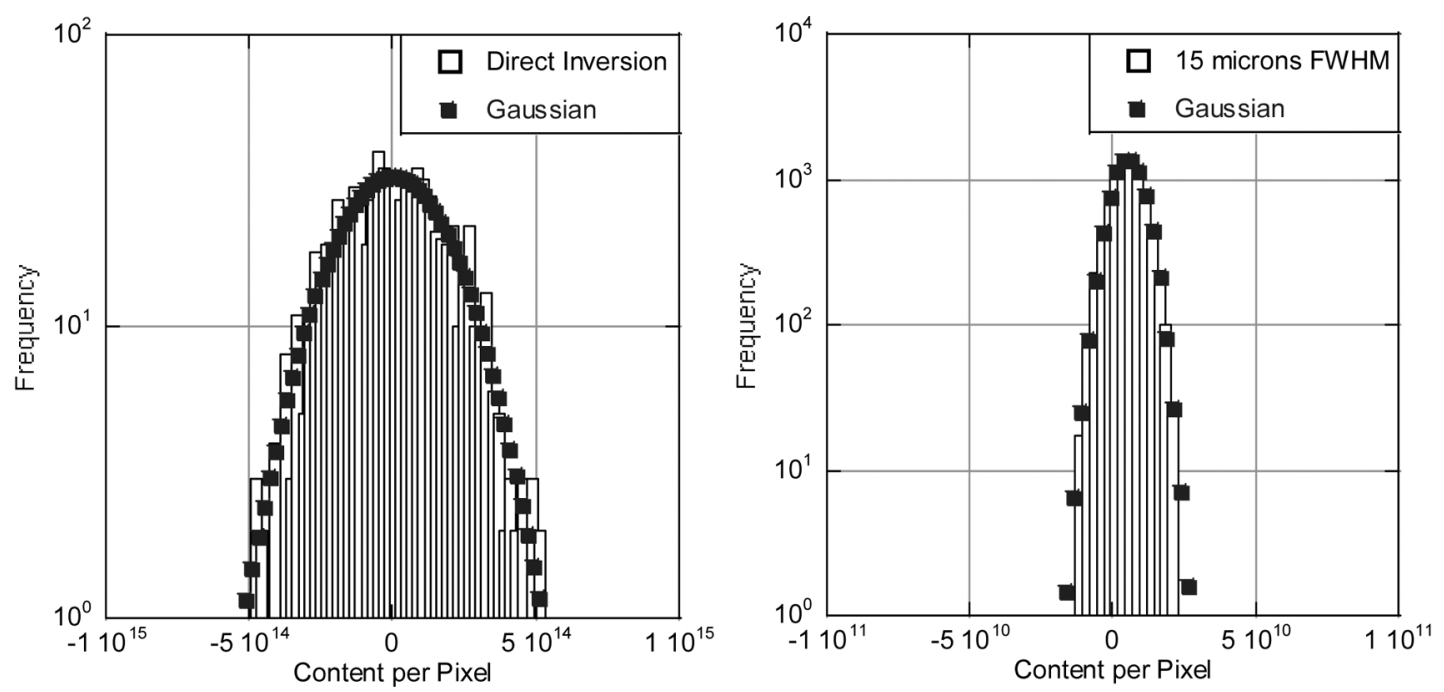

Figure 6.2: Histograms of the direct transmission pedestal region for the reconstructed source of the 5-micron square pinhole. Direct inversion (left) and after a 15 micron FWHM low-pass Gaussian filter. Notice the change in the horizontal scale.

\subsection{Resolution}

The NIS is required to produce images with a resolution of 10 microns at the source, but it does not establish the way this resolution is to be measured. In optics resolution has been traditionally defined as the minimum distance between two point sources so that they appear separate in the image plane. This definition is quantified by using Rayleigh's criterion which says that the angular separation $\theta$ between two point sources imaged though a circular aperture of diameter $d$, using light of wavelength $\lambda$, is given by

$$
\sin \theta=1.22 \frac{\lambda}{d}
$$

In neutron imaging, as in other kinds of radiological imaging techniques, there is no clear value of $d$ due to the high penetrating power of the radiation, and for particles, 
no practical wavelength can be quoted. This has given rise to a convention in some cases that the resolution should be quoted in terms of the FWHM of the PSF. This assumes that the PSF is small enough that no additional deconvolution is required; this is the case of x-ray and $\gamma$-ray imaging, where very good collimation can be achieved. However, as was shown in chapter four, the PSFs for the NIS are large compared to the desired resolution; so deconvolution becomes necessary.

Another way of defining the resolution of a system is as the maximum width of a smoothing filter that keeps a test feature recognizable by a trained observer. This particular definition was used to generate the design requirements for the NIS and in the failure mode analysis report[33]. This definition is arbitrary and depends on the shape of the filter used, usually a Gaussian, which is not a good representation of the PSFs. For the recording of a real image, no test feature is available, making the filter width determination problematic.

For this study a new resolution definition based on the Rayleigh's criterion will be used. In optics two point sources are said to be resolved when the first maxima of the airy pattern of one falls on the first null of the other one. Based on equation 6.3 and the intensity of the difraction pattern of a circular aperture

$$
I(\theta)=I_{o}\left(\frac{2 J_{1}(k a \sin \theta)}{k a \sin \theta}\right)^{2}
$$

where $J_{1}$ is the Bessel function of first kind of order one, $k$ is the wave number $k=2 \pi / \lambda$ and $a$ is the aperture radius $a=d / 2$, it can be shown that the intensity of 


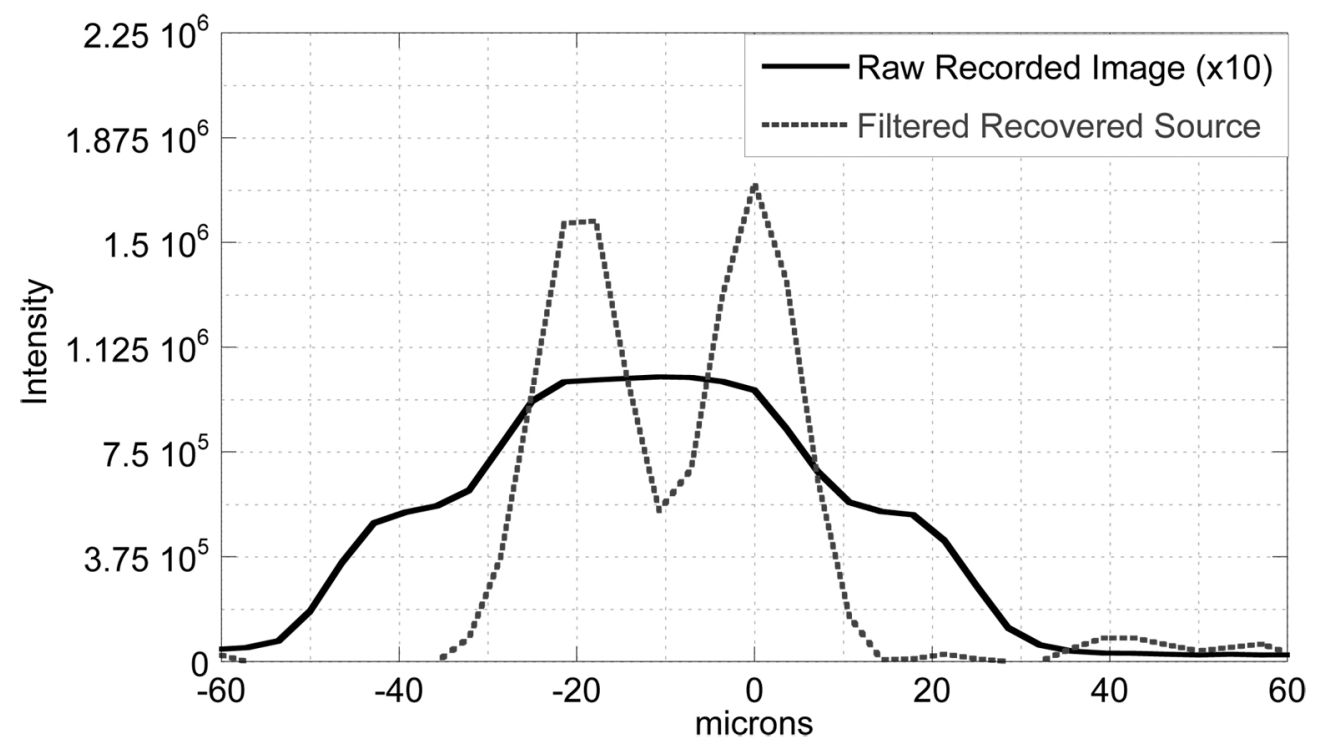

Figure 6.3: Vertical lineout through the center of figure 5.16. Two 10-micron discs set 20 microns apart appear unresolved in the 50-micron square pinhole raw recorded image, but are fully resolved after deconvolution.

the valley between the two maxima is given by

$$
\frac{8}{\pi^{2}}=0.8105
$$

For the NIS two source objects are said to be resolved after deconvolution when the depth of the dip between them is $19 \%$ of the maxima. This definition stresses the importance of deconvolution, since two sources can appear unresolved in the raw recorded image, but be completely resolved after deconvolution and filtering. This can be seen in figure 5.16, which shows four 10-micron discs separated at least 20 microns imaged through the 50-micron square pinhole. The plot in figure 6.3 shows the vertical lineouts through the center before and after deconvolution.

In the case of a simulated macroscopic neutron source distribution, like the differ- 
ence downscattered, it is more complicated to assess the achievable resolution, since the features are smaller (source elements are 2.5 microns squares) and have varying intensities. In a real neutron imaging experiment the actual source is not known, which forces the development of an off-line system resolution calibration.

\subsection{Signal-to-Noise Ratio}

As with resolution, there are multiple ways to define the SNR of an image. Historically in neutron imaging the SNR has been simply defined as the maximum source pixel value over the standard deviation of the background pixels. This definition has been used to quantify the quality of recovered sources based on images recorded at Nova by Lerche et.al.[60], and at Omega by Disdier et.al.[49] as recently as 2005. This definition is adequate for these experiments, since the sources produced at these two facilities are small, and due to the low neutron yield and low optical magnification, they do not contain much structural detail. These bright primary neutron sources extend over very few pixels and most of the final image is just background generated by the source recovery process.

It is also a common practice that before calculating the standard deviation of the background in the recovered source, all the negative valued pixels are replaced by zeros. As it was shown in section 5.2.2.1, the negative values are a normal consequence of the processing in frequency space. Changing these values to zero, biases the background histogram and artificially boosts the SNR. In order to have a more 
accurate measure of the image quality the negative values should be left unchanged.

This definition tends to produce very high SNR values when a wide (in real space) low-pass filter is applied to the image. This is due to the smoothing of the large background area; which reduces the standard deviation of the pixel distribution, increasing the SNR. This can be a misleading practice since high SNR values are seen as good, but as was shown in figure 5.5, the wider the low-pass filter is, the more source detail is lost, making the image useless.

The high neutron yields, large compressed cores and high magnification available at the NIF will combine to generate downscattered images that show complex structure. Using the standard 250 microns square fiber array, these sources can occupy around $40 \times 40$ pixels. At the same time the design requirement calls for the SNR to be measured at the $20 \%$ contour, not at the maximum. This shows that a new SNR definition is required. In this study the SNR is defined as the simple average of the pixel values inside the contour of interest, over the standard deviation of this sample.

Figure 6.4 shows the recovered downscattered source for the 5-micron and 50micron square pinholes. The $20 \%$ contour $^{1}$ has been artificially enhanced to distinguish it from its surroundings. These regions contain 218 and 115 pixels respectively, which are small samples to get an accurate estimate of the standard deviation. Most importantly, in apertures with lower magnifications the relative size of the pixel is large compare to the source, and the contour is not as meaningful as in the high

\footnotetext{
${ }^{1}$ The $20 \%$ contour contains values greater than $10 \%$ and lower than $20 \%$ of the image maximum.
} 

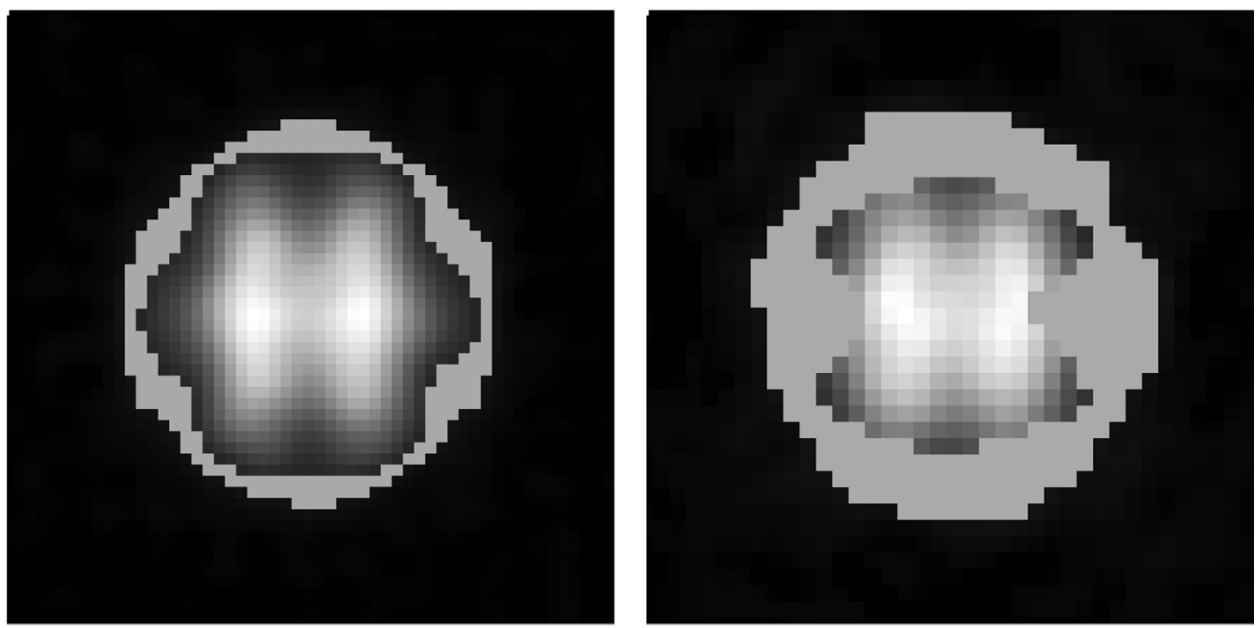

Figure 6.4: $20 \%$ contour (highlighted) of the recovered filtered downscattered source for the 5-micron (left) and 50-micron square pinhole apertures. Source orientation is the same in both images.

magnification case.

\subsection{Surrogate Sources}

The end-to-end model can be used to obtain an accurate estimate of the achievable resolution and SNR for each LOS configuration. MCNPX can process a surrogate source with enough pixels and an intensity equivalent to the $20 \%$ contour, generating a raw image that is deconvolved with the same parameters as the downscattered source distribution. The recovered surrogate source has the same noise characteristics as the contour of interest, providing a reliable metric, which is not available directly from the real recovered source distribution.

Two surrogate sources were used for this study. The first one was a flat rectangular 

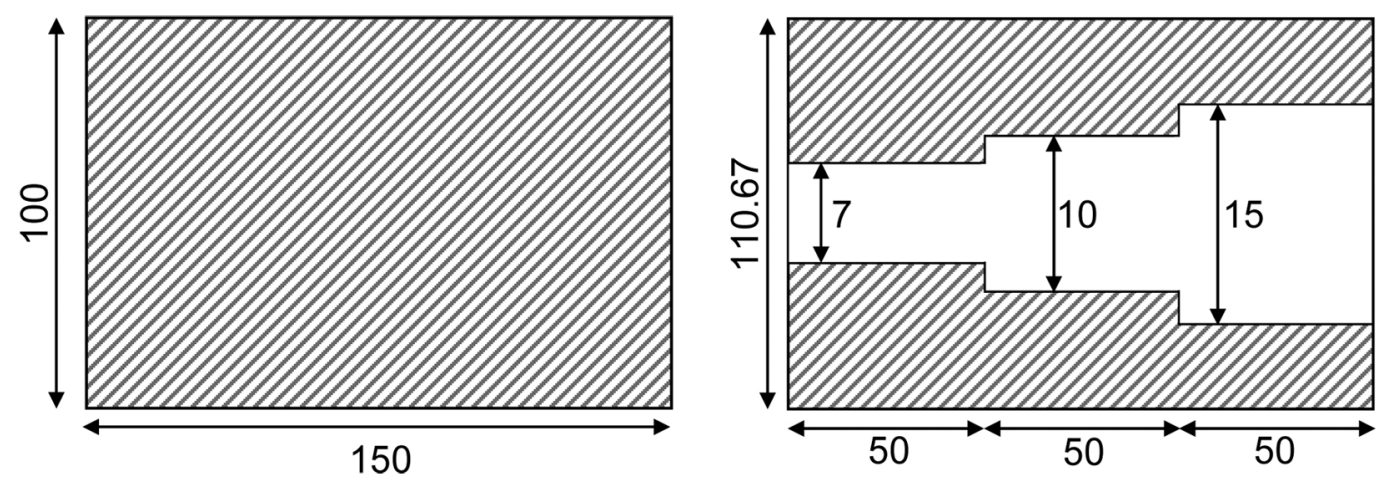

Figure 6.5: Construction details of the image quality surrogate sources used in MCNPX. The neutrons are generated uniformly from the crosshatched regions. Measurements in microns. Drawings not to scale.

slab $100 \times 150$ microns oriented facing the LOS, with the long side parallel to the $Y$ axis. The neutrons were generated uniformly across the rectangular surface. The second source was a slightly larger slab with three gaps through the middle; the widths of the gaps were 7,10 and 15 microns. The neutrons were produced in the two flat regions outside the gaps. The length of the slab was kept constant at 150 microns, but the height was increased to 110.67 in order to compensate for the area of the gaps and to achieve the same intensity as the flat slab. Figure 6.5 shows the construction details of both surrogate sources.

The SNR was calculated from the flat slab by sampling the plateau, a rectangular area of uniform intensity located close to its center. This source can be used to illustrate the effect of a low-pass filter on the image quality and the SNR. Figure 6.6 shows the 50-micron square pinhole slab source after three Gaussian filters: 7, 10 and 15 microns FWHM, the corresponding SNR values are: 11.67, 13.97 and 14.85. 

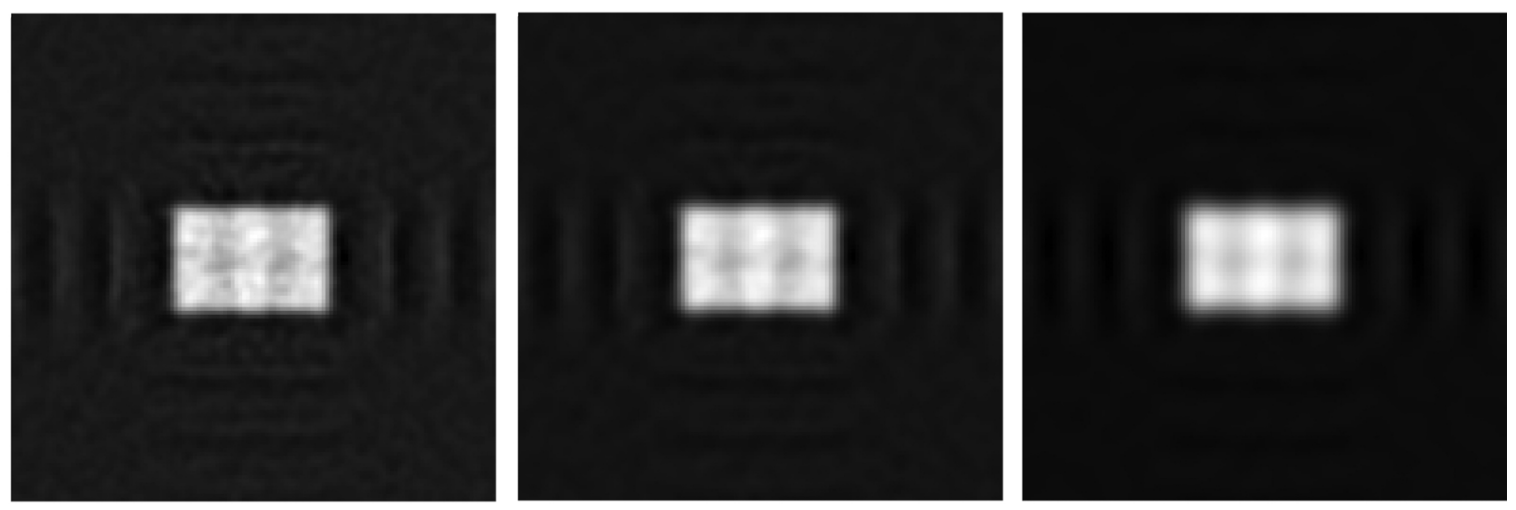

Figure 6.6: 50-micron square pinhole filtered recovered flat slab source. Filter FWHM in microns: 7 (left), 10 (center) and 15. The corresponding SNR values on the slab are: 11.67, 13.97 and 14.85. A 450 micron square $\mathrm{FoV}$ is shown. Images shown in a linear grayscale.

Although the 15 micron FWHM filter has the highest SNR, the edges of the flat source have been slightly rounded and smoothed.

The system's resolution was studied with the gap source. The ability of a particular LOS configuration to resolve the test gaps can be verified by taking vertical lineouts through the three sections and checking to see whether they satisfy Rayleigh's criterion. Figure 6.7 shows the deconvolved gap source for the 100-micron mini-penumbral aperture after low-pass filtering (same widths as before), along with averaged (over 5 pixels) vertical lineouts of each gap. This figure shows that the aperture has a resolution of 7 microns or better for any of the filters used. All the LOS configuration studied resolve the 7 micron gap with a 7 micron FWHM filter.

Table 6.1 collects the SNR for each aperture; these values were obtained using the same regularization parameter (RP) and low-pass filter FWHM used in sections 5.2.2.1 and 5.2.3 to generate the reconstructed downscattered sources. 

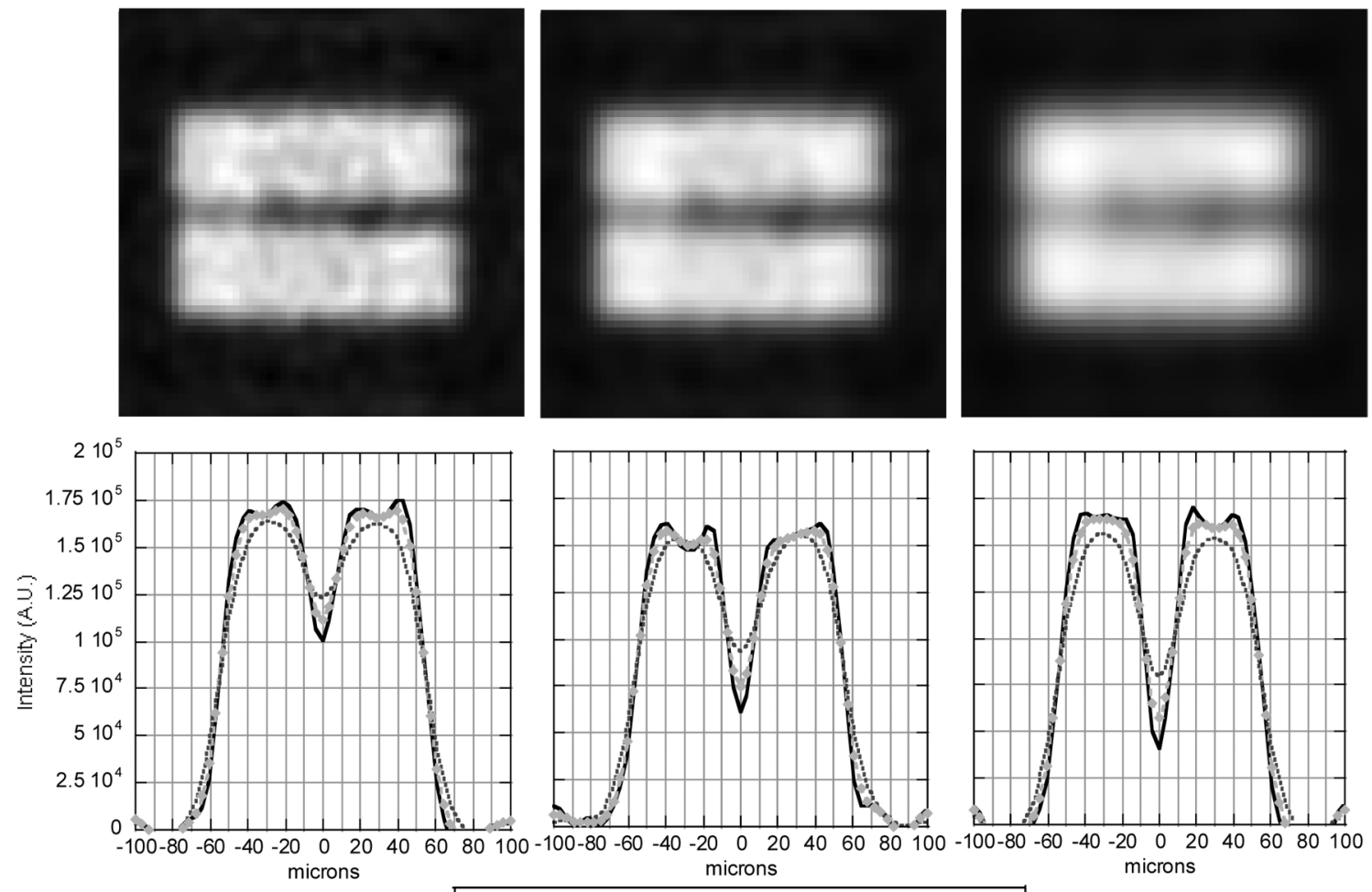

7 FWHM $--\$--10$ FWHM

Figure 6.7: 100-micron mini-penumbral aperture recovered gap source. Real space images and vertical lineouts through the three gaps for each filter. Filter FWHM in microns: 7 (left), 10 (center) and 15. A 210 micron square FoV is shown. Images shown in a linear grayscale. 


\begin{tabular}{lccc}
\hline \hline Aperture Type & $\begin{array}{c}\text { RP } \\
\text { (\% of max) }\end{array}$ & $\begin{array}{c}\text { Filter FWHM } \\
\text { (microns) }\end{array}$ & $\begin{array}{c}\text { SNR } \\
\text { (a.u.) }\end{array}$ \\
\hline 5-micron Square Pinhole & 10.000 & 9 & 8.83 \\
50-micron Square Pinhole & 5.000 & 5 & 10.04 \\
Triangular Wedge & 5.000 & 10 & 10.65 \\
50-micron Mini-Penumbral & 1.000 & 10 & 5.58 \\
100-micron Mini-Penumbral & 2.500 & 5 & 11.85 \\
Annular at 40 m & 15.000 & 5 & 9.78 \\
Annular at 28 m & 5.000 & 5 & 7.11 \\
Penumbral at 40 m & 0.200 & 6 & 6.47 \\
Penumbral at 28 m & 0.035 & 7 & 6.57 \\
\hline \hline
\end{tabular}

Table 6.1: Summary of the SNR expected for all apertures based on the $20 \%$ contour intensity of the recovered difference downscattered source and the surrogate sources. 


\section{Chapter 7}

\section{Concluding Remarks and}

\section{Suggestions for Further Work}

Neutron imaging is a challenging diagnostic technique for the complex scientific effort that is laser driven inertial confinement fusion. At the time of this study the first ignition experiments at the National Ignition Facility (NIF) are two years away, and the suite of target diagnostics is steadily moving through conceptual and engineering design reviews. The main goal of the present study was to shine some light on the Neutron Imaging System (NIS) design by probing the parameter space through detailed numerical simulations and image processing, and by experimentally characterizing the response of one of the components. The results of the present study can be used as the basis for the definitive NIS design. The most important findings are presented bellow; they are divided by major issues. There are, of course, several 
areas that require further research, modeling and analysis, and as neutron imaging experiments are carried out, possibly some new ones will arise. Some suggestions for future work are collected in the final part of this chapter.

\subsection{Scintillator Characterization}

The light emission decay measurements indicate that all the samples studied show a long lived (> $300 \mathrm{~ns}$ ) decay component. This translates into an afterglow, caused by the primary neutrons, in the downscattered image, for both the 28 and $40 \mathrm{~m}$ lines of sight. An effective solution to the afterglow contamination is to record two images, the 13 - $15 \mathrm{MeV}$ primary and the $6-10 \mathrm{MeV}$ downscattered, and then subtract a decayed version of the former from the latter.

Based on the decay profiles, measured sensitivity and photon gain, the best two candidates for a scintillator array are: plastic fibers of EJ232 (BC422) and capillaries filled with EJ399B.

\section{$7.2 \quad$ End-to-End Model}

The NIS end-to-end model proved to be a very powerful tool in understanding the effect of the LOS configuration (aperture type and size and optical magnification) in the shape and quality of the recorded images. The end-to-end model is flexible and can be used to study alternative LOS configurations and neutron source distributions, 
such as a pure $P_{4}$ or multimode driver asymmetries.

Due to the low intensity of the downscattered source, not all simple apertures can be used directly. The square pinhole, triangular wedge and mini-penumbral geometries require the use of multi-aperture arrays in order to increase the number of counts per pixel, and produce a useful recorded image. The actual size of the array will depend on the length of the LOS and, most importantly, on the actual size of the scintillator array. The annular and penumbral apertures can generate useful images directly.

The MCNPX model of the pixelated scintillator array shows that the recoil proton range is not a significant contribution to the PSF of system. Most of the light is produced inside the fiber where the original $(n, p)$ interaction takes place. The accuracy of this prediction depends on the actual construction details of the detector array.

\subsection{Image Processing and Source Recovery}

Only the 5-micron square pinhole, which has a very small PSF, generates recorded images that are readily usable. The other apertures studied produce images that require deconvolution in order to obtain a usable source representation. The simple frequency-space m-regularization method is effective in removing the PSF blur and recovering source distributions very close to the original when an appropiate low-pass filter is applied. The inverse nature of the m-regularization method partially enhances the noise present in the recorded image, making the raw reconstructed source barely 
visible in real space, leading to the need for filtering.

The isoplanatic approximation is a good alternative for the NIS. As long as the source is kept centered in the FoV, the variations of intensity can be corrected in post processing. According to figure 5.15, the $28 \mathrm{~m} \mathrm{LOS} \mathrm{seems} \mathrm{to} \mathrm{have} \mathrm{more} \mathrm{uniform}$ intensity across the FoV, but each aperture has a unique profile and must be considered independently. Shape distortions induced by the simple PSF are harder to correct, but preliminary studies by Lantuejoul[79] indicate that penumbral apertures are less sensitive to geometric aberrations than annular apertures; this behavior is thought to extend to the mini-penumbral arrays. The treatment of a position dependant PSF is mathematically complex[80] and in its implementation, making the single PSF approximation more attractive.

\subsection{Image Quality and Aperture Selection}

All the LOS configurations studied can generate a recovered source, but with varying quality. The two image quality parameters, resolution and SNR at the $20 \%$ contour, can be effectively quantified using the two surrogate sources.

Using Rayleigh's criterion as basis for resolution, the simulations show that all the configurations can successfully resolve at least 7 microns. Further tests with narrower gaps are necessary to establish the true resolution limit. The SNR results are not as straightforward. As shown by the simulations of section 6.2 , the image noise is caused by the number of neutrons entering a pixel. Since the size of the detector element is 
constant (250 microns square), the $28 \mathrm{~m}$ LOS should have higher SNR, which is the case, except for the annular aperture. The image quality, and thus the performance of the LOS configuration, should not be based solely on these two numerical values; the trained observer should judge the images as a whole. A systematic decision study should be conducted as it is being done with the failure mode analysis and diagnostic signatures.

Ultimately the aperture design decision will be based on manufacturing issues. This point aside, the 50-micron square pinhole and 100-micron mini-penumbral arrays are the best choices based on SNR and appearance. The Penumbral and Annular apertures show marginal SNR but this can be improved by using the filteredautocorrelation method.

\subsection{Areas of Further Work}

The effectiveness of the end-to-end model can be greatly enhanced by incorporating more design details about the aperture, scintillator array, the detector optical system, the CCD camera and the surrounding infrastructure. These elements affect the image statistics and can introduce aberrations that have not been taken into account in this study.

Construction details about the aperture and its fixture, the detector housing and imaging station (inside or outside the NIF's main building) are necessary to undertake detailed background studies that can provide essential information about the realistic 
recorded image noise load, and any possible flat-fielding problems due to scattered radiation. These issues are well known to experimental teams at Omega and should be addressed at the NIF before a design is finalized.

The actual scintillator array, plastic or liquid, needs to be characterized for sensitivity and light output angular distribution at several neutron energies. Measurements in the 6 to $10 \mathrm{MeV}$ range are important to better understand the light production in the downscattered image. If a plastic fiber design is used, the large numerical aperture claimed by the manufacturer needs to be carefully verified.

For this study the m-regularization method used an MCNPX-generated PSF, which did not have any imperfections, as it would be expected from any manufacturing process. The effectiveness of the m-regularization method depends on the perfect matching of the raw recorded image with the PSF; for the NIS the actual as-built PSF needs to be known. Several methods have been suggested for characterizing the aperture, some of them are: neutron radiography using an intense tunable source (like the 88-inch cyclotron at the Lawrence Berkeley Laboratory), high resolution ultra sound scanning, and using a high yield implosion at Omega, where the NIS aperture would produce an out-of-focus image of the source, but it would be uniformly illuminated, generating a PSF that can be compared to the as-designed MCNPX one.

The LOS configurations studied were all ideal; with perfectly aligned components, on-axis PSF, straight profiles and sources located at TCC. Once an aperture is chosen and in order to understand the impact of LOS imperfections on the recorded image 
and recovered source, a systematic study of the imperfection parameter space needs to be carried out. The main imperfections are: source location around the FoV, component alignment and aperture manufacturing imperfections.

An engineering issue that affects existing neutron imaging systems and that has not been fully addressed is alignment. A robust alignment infrastructure and procedure needs to be developed. Recent imaging campaigns at Omega have suffered from severe misalignment (internal and whole LOS). This is worrying since the Omega LOS, only $8 \mathrm{~m}$ long, is very short compared to the 28 or $40 \mathrm{~m}$ ones being considered for the NIS. As was shown in section 5.2.4, the isoplanatic approximation holds well for a FoV region with $r<50$ microns, any small misalignment would push the image outside this region, introducing intensity and, possibly, shape distortions.

The choice of an open neutron aperture, like a mini-penumbral array, would have a high impact on the alignment accuracy, since back illumination methods could be used instead of the current ones, which are based on retro-reflection.

The MCNPX model needs to be extended so it can accommodate full size pinhole arrays. A series of preliminary tests was conducted using the 100-micron minipenumbral in $3 \times 3$ and $7 \times 7$ configurations, the corresponding PSFs are shown in figure 7.1. In these arrays each aperture is pointed towards TCC and, due to the small angles involved, no shape distortions are visible in the recorded images. This is an advantage for algorithms at add up the individual cropped images to generate a single one. This type of algorithm is likely to suffer from registration error, i.e., a random 

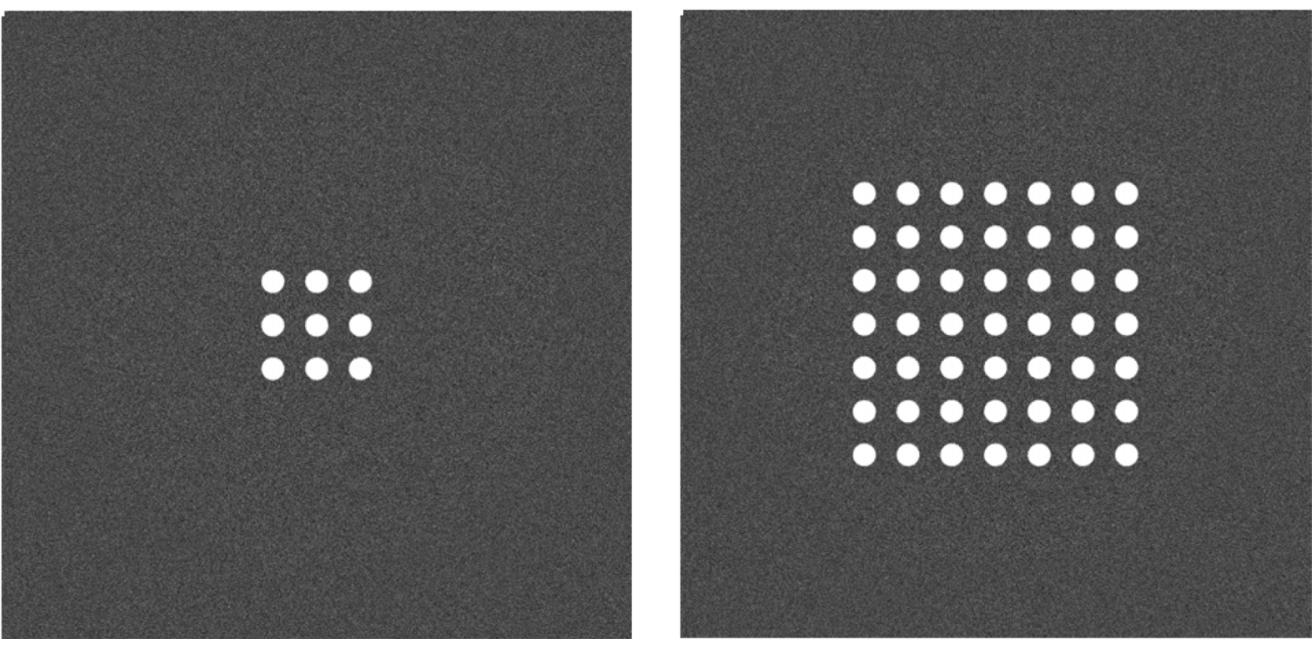

Figure 7.1: PSF of the $3 \times 3$ and $7 \times 7$ 100-micron mini-penumbral arrays. Images are shown uncropped $(1024 \times 1024$ pixels $)$ in a linear grayscale.

variation in the location of the center of each image. The effect of this error on the noise level of the final image needs to be studied in detail. Simple simulations show that the blurring introduced is small, and comparable to the smoothing generated by soft m-regularization.

The problems associated with the image addition process can be avoided if the whole $7 \times 7(10 \times 10$ for the $40 \mathrm{~m} \mathrm{LOS})$ image is used to recover the source. Preliminary tests using a $7 \times 7 \mathrm{PSF}$ and the same m-regularization algorithm produced a single centered source, but with very low image quality (see figure 7.2). Extensive study of this approach is needed, in particular it is necessary to understand how the direct transmission pedestal affects the whole-array deconvolution, it seems that the extra power introduced in the image by the larger array, masks the source information more than in the single aperture case. 

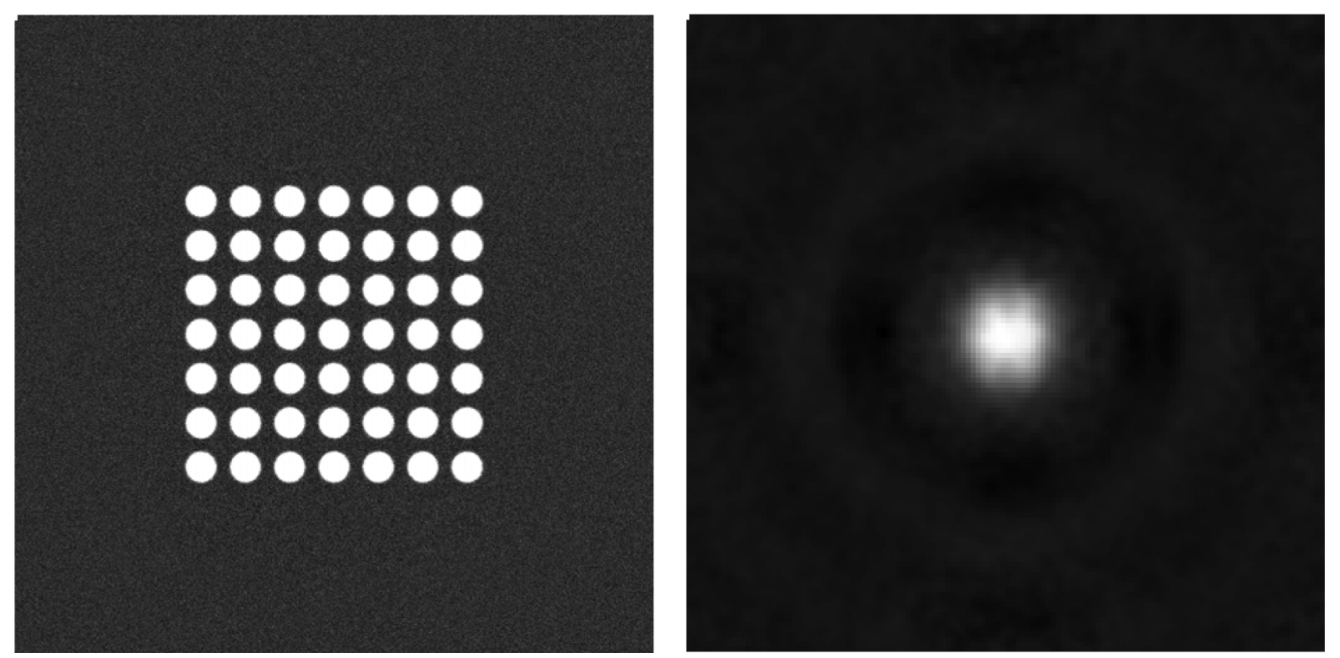

Figure 7.2: Difference Downscattered image (left) for the $7 \times 7$ 100-micron minipenumbral array. Right: m-regularized receovered source cropped to 128 pixels. Notice that the source is surrounded by some strange structure and not fully resolved, as was the case in the single aperture case. Images are shown in a linear grayscale.

The NIS can greatly benefit from more powerful source recovery algorithms, as exemplified by the filtered autocorrelation process used with the penumbral and annular apertures. Techniques that do not require a detailed PSF would be preferable to those, like the m-regularization, that need an exact representation. Some promising candidates are: entropy maximization, maximum likelihood and blind deconvolution. Whole-array source recovery can be explored using matched filters and random arrays[71]. The main problem with random arrays for neutron imaging is manufacturing. It is very complicated to achieve the required random structure given the neutron aperture thicknesses, but possibly EDM can be used to produce a random array of mini-penumbral apertures.

Looking into the future of experimental installations like the NIF, LMJ and Omega 
EP, neutron imaging can play an important role in the diagnosis of fast ignition targets and high yield capsules; additional lines of sight would open the door to neutron tomography and more powerful imaging studies, which will enhance our knowledge of the ICF physics and help us move closer to the goal of harnessing the energy of the stars for the benefit of all humanity. 


\section{References}

[1] K.S. Krane. Introductory Nuclear Physics. John Wiley \& Sons, Hoboken, NJ, 1988.

[2] R.D. Evans. The Atomic Nucleus. McGraw-Hill, New York, NY, 1955.

[3] S. Pfalzner. An Introduction to Inertial Confinement Fusion. Taylor \& Francis, Boca Raton, FL, 2006.

[4] A.A. Harms, K.F. Schoepf, G.H. Miley, and D.R. Kingdon. Principles of Fusion Energy. World Scientific, Singapore, 2000.

[5] G. Velarde, Y. Ronen, and J. M. Martínez-Val. Nuclear Fusion by Inertial Confinement: A Comprenhensive Treatise. CRC Press, Boca Raton, FL, 1992.

[6] G.H. Miley, H. Towner, and N. Ivich. Technical Report COO-2218-17 Nuclear Engineering, University of Illinois, Urbana-Champaign, IL, 1974.

[7] J. Rand McNally, Jr., K.E. Rothe, and R.D. Sharp. Fusion reactivity graphs and 
tables for charge and particle reactions. Technical Report ORNL/TM-6914, Oak Ridge National Laboratory, Oak Ridge, TN, 1979.

[8] D. Keefe. Inertial confinement fusion. Annual Review of Nuclear and Particle Science, 32:391, 1982.

[9] G.H. Miley. Fusion Energy Conversion. American Nuclear Society, La Grange Park, IL, 1976.

[10] J.D Lawson. Some criteria for a power producing thermonuclear reactor. Proceedings of the Physical Society. Section B, 70:6, 1957.

[11] S. Atzeni and J. Meyer ter Vehn. The Physics of Inertial Fusion. Oxford University Press, Oxford, 2004.

[12] M.D. Rosen. The physics issues that determine inertial confinement fusion target gain and driver requirements: a tutorial. Physics of Plasmas, 6:1690, 1999.

[13] L. Spitzer. Physics of Fully Ionized Gases. Interscience Publishers, New York, NY, 1962.

[14] N.G. Basov. IEEE Journal of Quantum Electronics, QE-4:864, 1968.

[15] N.G. Basov and D.N. Krohkin. Proceedings of the Third International Congress in Quantum Electronics. Columbia University Press, New York, NY, 1963.

[16] J. Nuckolls, L. Wood, A. Thiessen, and G. Zimmerman. Laser compression 
of matter to super-high densities: Themonuclear (CTR) applications. Nature, 239:139, 1972.

[17] E.S. Bliss, J.A Glaze, K.R. Manes, J.E. Murray, and F. Rainer. Cyclops laser system. Technical Report UCRL 5002175 Laser Program Annual Report 1975, Lawrence Livermore National Laboratory, Livermore, CA, 1975.

[18] W.W. Simmons, D.R. Speck, and J.T. Hung. Argus laser system: Performance summary. Applied Optics, 17:999, 1978.

[19] D.R. Speck et al. The Shiva laser-fusion facility. IEEE Journal of Quantum Electronics, QE-9:1599, 1981.

[20] W. Seka, S.D. JAcobs, J.E. Rizzo, R. Boni, and R.S. Craxton. Demonstration of high efficiency third harmonic conversion of high power Nd-glass laser radiation. Optics Communications, 34:469, 1980.

[21] R.S. Craxton. Theory of high efficiency third harmonic conversion of high power Nd-glass laser radiation. Optics Communications, 34:474, 1980.

[22] E.M. Campbell, J.T. Hunt, E.S. Bliss, D.R. Speck, and R.P. Drake. Nova experimental facility. Review of Scientific Instruments, 57:2101, 1986.

[23] R. Sawicki, J. Bowers, R. Hackel, D. Larson, K. Manes, and J. Murray. Engineering the National Ignition Facility. Fusion Technology, 34:1105, 1998. 
[24] W.J. Hogan, E.I. Moses, B.E. Warner, M.S. Sorem, and J.M. Soures. The National Ignition Facility. Nuclear Fusion, 41:567, 2001.

[25] G.H. Miller, E.I. Moses, and C.R. Wuest. The National Ignition Facility:enabling fusion ignition for the 21st century. Nuclear Fusion, 44:228, 2004.

[26] V. Yu. Glebov et al. Development of nuclear diagnostics for the National Ignition Facility. Review of Scientific Instruments, 77:10E715, 2006.

[27] M.J. Moran et al. PROTEX: a proton-recoil detector for inertial confinement fusion neutrons. Review of scientific instruments, 76:023506, 2005.

[28] V. Yu. Glebov et al. Carbon activation diagnostic for tertiary neutron measurements. Review of scientific instruments, 74:1717, 2003.

[29] J.A Frenje et al. A neutron spectrometer for precise measurements of DT neutrons from 10 to $18 \mathrm{MeV}$ at OMEGA and the National Ignition Facility. Review of scientific instruments, 72:854, 2001.

[30] R. Tommasini et al. High energy X-ray imager for inertial confinement fusion at the National Ignition Facility. Review of scientific instruments, 77:10E301, 2006.

[31] T.R. Boehly et al. Initial performance results of the OMEGA laser system. Optics Communications, 133:495, 1997. 
[32] J. B. Marion and S. T. Thorton. Classical Dynamics of Particles and Systems. Saunders College Publishing, Forth Worth, Fourth edition, 1995.

[33] C. Cerjan, S. Haan, S. Hatchett, and J. Koch. Failure modes and diagnostic signatures working group - Ignition diagnostics requirements update. Technical Report UCRL-TR-229780, Lawrence Livermore National Laboratory, Livermore, CA, 2007.

[34] G.F. Knoll. Radiation Detection and Measurement. John Wiley \& Sons, Hoboken, NJ, Third edition, 2000.

[35] M.J. Moran. Personal communication.

[36] R.A. Lerche and G.E. Sommargren. Collimator design for neutron imaging of laser-fusion targets. Technical Report UCID 19317, Lawrence Livermore National Laboratory, Livermore, CA, 1981.

[37] K.A Nugent and B. Luther-Davies. Penumbral imaging of high energy x-rays from laser-produced plasmas. Optics Communications, 49:393, 1984.

[38] K.A Nugent and B. Luther-Davies. Application of penumbral imaging to thermonuclear neutrons. Journal of Applied Physics, 58:2508, 1985.

[39] K.A Nugent and B. Luther-Davies. Penumbral neutron imaging - optimization and simulation. Journal of Applied Physics, 60:1289, 1986. 
[40] D. Ress, R.A. Lerche, R.J. Ellis, S.M. Lane, and K.A Nugent. Neutron imaging of laser fusion targets. Science, 241:956, 1988.

[41] D. Ress, R.A. Lerche, R.J. Ellis, S.M. Lane, and K.A Nugent. Neutron imaging of inertial confinement fusion targets at Nova. Review of Scientific Instruments, 59:1694, 1988.

[42] J.-P. Garçonnet et al. Neutron penumbral imaging of inertial confinement fusion targets. Laser and Particle Beams, 12:563, 1994.

[43] O. Delage, J.-P. Garçonnet, D. Schirmann, and A. Rouyer. Neutron penumbral imaging of inertial confinement fusion targets at Phébus. Review of Scientific Instruments, 66:1205, 1995.

[44] O. Delage et. al. High spatial resolution imaging system for high energy neutrons in inertial confinement fusion experiments. In O. Delage, E. Munro, and J.A. Rouse, editors, Charged Particle Detection, Diagnostics and Imaging, volume 4510 of Procedings of SPIE. SPIE, 2001.

[45] R.A. Lerche et al. Neutron images recorded with high-resolution bubble detectors. Review of Scientific Instruments, 74:1709, 2003.

[46] L. Disdier et al. Neutron imaging of ICF target plasmas. Review of Scientific Instruments, 74:1832, 2003. 
[47] C.R. Christensen et al. First results of pinhole neutron imaging of inertial confinement fusion. Review of Scientific Instruments, 74:2690, 2003.

[48] G.P. Grim et al. Progress on neutron pinhole imaging for inertial confinement fusion experiments. Review of Scientific Instruments, 75:3572, 2004.

[49] L. Disdier et. al. Inertial confinement fusion neutron images. Physics of Plasmas, 13:056317, 2006.

[50] C.A. Barrera. Characterization of scintillator materials for downscattered neutron imaging. Master's thesis, University of California at Berkeley, 2004.

[51] L. Disdier et al. Capillary detector with deuterated scintillator for inertial confinement fusion neutron images. Review of Scientific Instruments, 75:2134, 2004.

[52] J. B. Birks. The Theory and Practice of Scintillation Counting. Pergamon Press, Oxford, 1964.

[53] D.V. O'Connor and D. Phillips. Time-Correlated Single Photon Counting. Academic Press, London, 1984.

[54] P. B. Lyons et. al. Sub-nanosecond plastic scintillators. IEEE Transactions on Nuclear Science, 24:177, 1977.

[55] J. M. Flournoy. Measurement of decay times of fast scintillator fibers. Radiation Physics and Chemistry, 41:389, 1993. 
[56] W.R. Leo. Techniques for Nuclear and Particle Physics Experiments. SpringerVerlag, New York, Second edition, 1994.

[57] D. L. Smith, R. G. Polk, and T. G. Miller. Measurement of the response of several organic scintillators to electrons, protons and deuterons. Nuclear Instruments and Methods, 64:157, 1968.

[58] D. L. Smith and R. L. Craun. Analysis of response data for several organic scintillators. Nuclear Instruments and Methods, 80:239, 1970.

[59] Bicron. General Catalog, 1993.

[60] R.A. Lerche et al. Neutron penumbral imaging of laser-fusion targets. Laser and Particle Beams, 1:99, 1991.

[61] D.B. Pelowitz Editor. MCNPX user's manual Version 2.5.0. Technical Report LA-CP-05-0369, Los Alamos National Laboratory, April 2005.

[62] G.W. McKinney. Personal communication.

[63] Proton range-energy tables for 63 compounds. Atomic Data and Nuclear Data Tables, page 147, 1982.

[64] H.H. Andersen and J.F. Ziegler. Hydrogen: Stopping Powers and Ranges in all Elements, Vol. 3. Pergamon Press, New York, 1977.

[65] J. D. Gaskill. Linear Systems, Fourier Transforms and Optics. John Wiley \& Sons, New York, 1978. 
[66] R. N. Bracewell. The Fourier Transform and its Applications. McGraw-Hill, New York, Second edition, 1978.

[67] J. W. Cooley and J. W. Tukey. An algorithm for the machine computation of complex Fourier series. Mathematics of Computation, 19:297, 1965.

[68] K. R. Castleman. Digital Image Processing. Prentice Hall, Upper Saddle River, 1996.

[69] R. C. Gonzalez and R. E. Woods. Digital Image Processing. Prentice Hall, Upper Saddle River, Second edition, 2002.

[70] mathsoft. MathCAD 11 User's Guide, 2002.

[71] H. H. Barrett and W. Swindell. Radiological Imaging. Academic Press, San Diego, 1981.

[72] P. A. Jansson. Deconvolution of Images and Spectra. Academic Press, San Diego, Second edition, 1997.

[73] R.H.T. Bates and M.J. McDonnell. Image Restoration and Reconstruction. Oxford University Press, Oxford, 1986.

[74] H. Stark. Image Recovery: Theory and Application. Academic Press, San Diego, 1987.

[75] M. Bertero and P. Boccacci. Introduction to Inverse Problems in Imaging. Institute of Physics Publishing, Bristol, 1998. 
[76] R.G. Simpson et. al. Digital processing of annular coded-aperture imagery. $O p$ tical Engineering, 14:490, 1975.

[77] A. Rouyer. A new simple method for decoding penumbra image: The filtered autocorrelation. Review of Scientific Instruments, 74:1234, 2003.

[78] M. Moran et.al. Downscattered neutron imaging. Review of Scientific Instruments, 75:3592, 2004.

[79] I. Lantuejoul. Personal communication.

[80] A. W. Lohmann and D. P. Paris. Space-variant image formation. Journal of the Optical Society of America, 55:1007, 1965. 